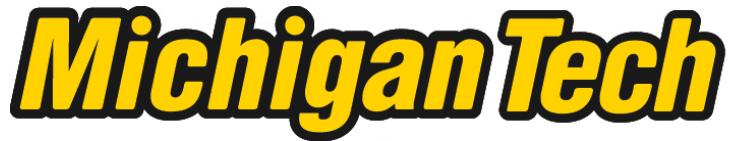 \\ Michigan Technological University Create the Future Digital Commons @ Michigan Tech
}

Dissertations, Master's Theses and Master's Reports - Open

Dissertations, Master's Theses and Master's

Reports

2011

\section{Omega-3 fatty acids and the neurovascular responses to mental stress in humans}

Christopher Elmer Schwartz

Michigan Technological University

Follow this and additional works at: https://digitalcommons.mtu.edu/etds

Part of the Biology Commons

Copyright 2011 Christopher Elmer Schwartz

\section{Recommended Citation}

Schwartz, Christopher Elmer, "Omega-3 fatty acids and the neurovascular responses to mental stress in humans", Dissertation, Michigan Technological University, 2011.

https://doi.org/10.37099/mtu.dc.etds/194

Follow this and additional works at: https://digitalcommons.mtu.edu/etds

Part of the Biology Commons 


\title{
OMEGA-3 FATTY ACIDS AND THE NEUROVASCULAR RESPONSES \\ TO MENTAL STRESS IN HUMANS
}

By

\author{
Christopher Elmer Schwartz
}

\begin{abstract}
A DISSERTATION
Submitted in partial fulfillment of the requirements for the degree of DOCTOR OF PHILOSOPHY

(Biological Sciences)
\end{abstract}

MICHIGAN TECHNOLOGICAL UNIVERSITY

2011

(c) 2011 Christopher E. Schwartz 
This dissertation, "Omega-3 Fatty Acids and the Neurovascular Responses to Mental Stress in Humans," is hereby approved in partial fulfillment of the requirements for the Degree of DOCTOR OF PHILOSOPHY IN BIOLOGICAL SCIENCES.

Department of Biological Sciences

Signatures:

Dissertation Advisor

Jason R. Carter, PhD

Department Chair

K. Michael Gibson, PhD

Date 


\section{Table of Contents}

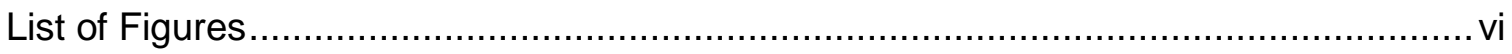

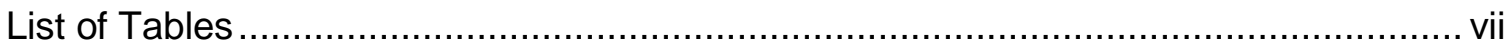

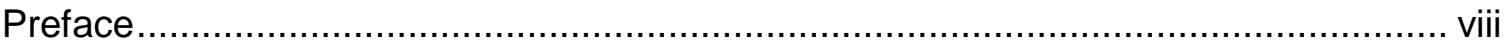

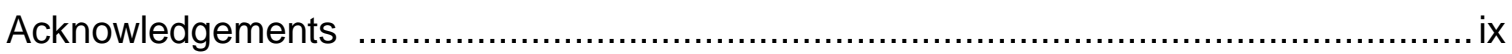

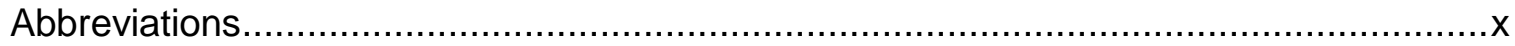

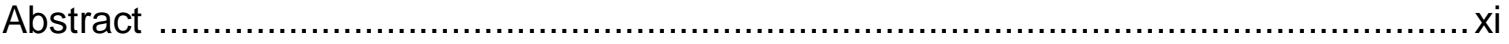

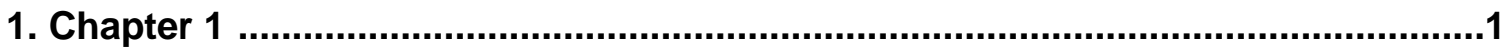

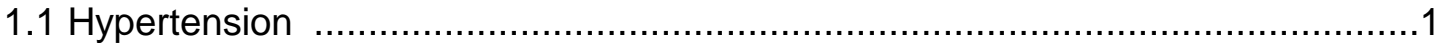

1.2 Ways to Assess Autonomic Activity ......................................................

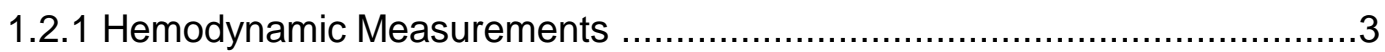

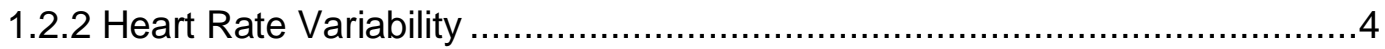

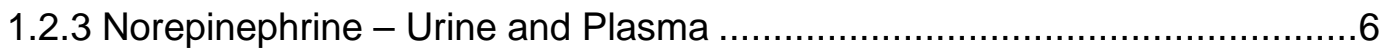

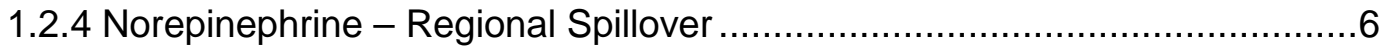

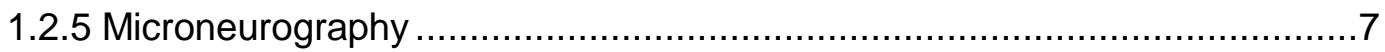

1.3 Neural Responses to Mental Stress ........................................................

1.4 Cardiovascular Responses to Mental Stress............................................

1.5 Mental Stress and Cardiovascular Disease ............................................. 12

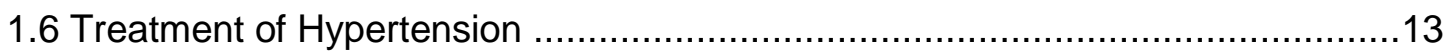

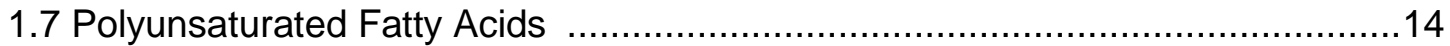

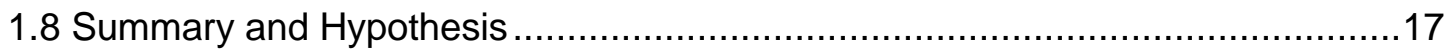

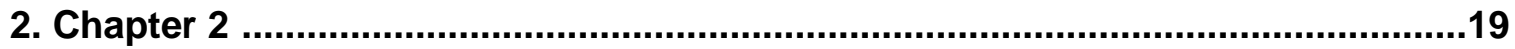

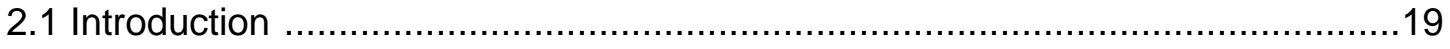

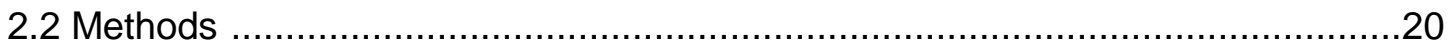

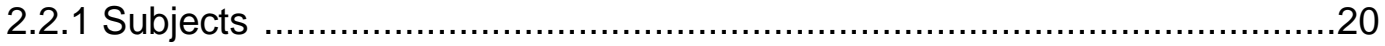

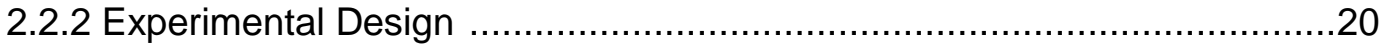




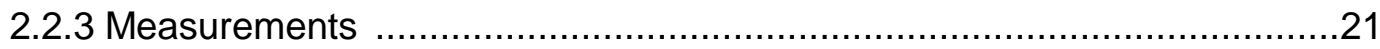

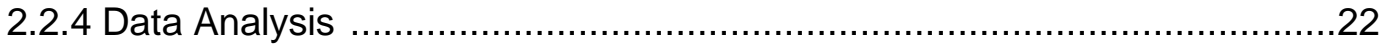

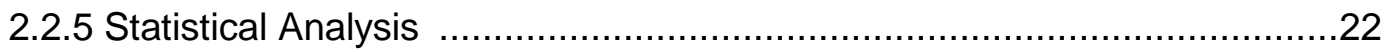

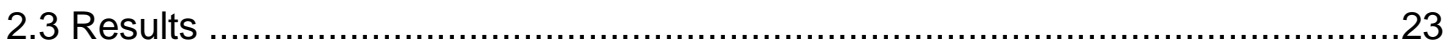

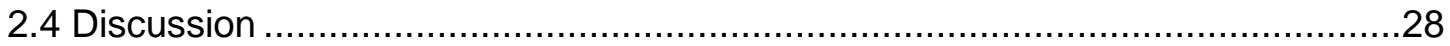

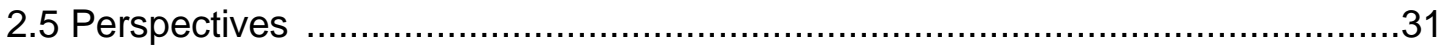

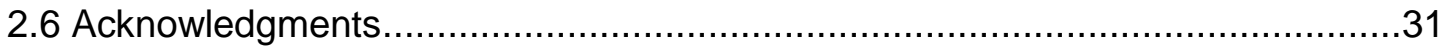

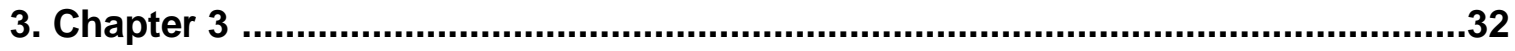

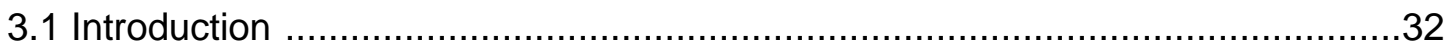

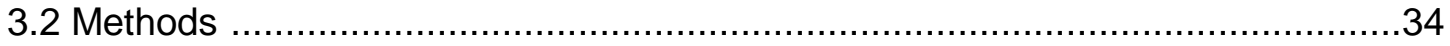

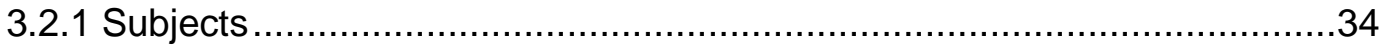

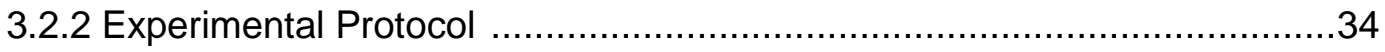

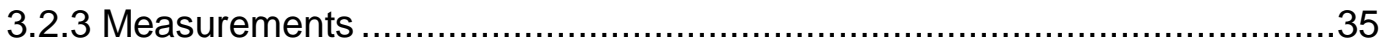

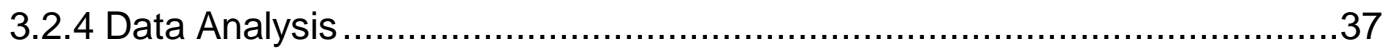

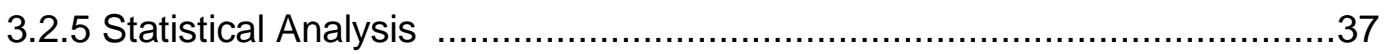

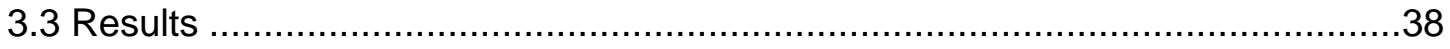

3.3.1 Resting Baseline Measurements for Normotensive and Prehypertensive Subjects .............................................. 38

3.3.2 Resting Measurements for Combined Non-Hypertensive Subjects

3.3.3 Hemodynamic Responses to Mental Stress in Normotensive and Prehypertensive Subjects

3.3.4 Sympathetic Nerve Responses to Mental Stress in Normotensive and Prehypertensive Subjects .............................................. 46

3.3.5 Peripheral Vascular Responses to Mental Stress in Normotensive and Prehypertensive Subjects

3.3.6 Responses to Mental Stress for Combined Non-Hypertensive Subjects 
3.4.1 Resting Neurovascular Responses to Omega-3

Supplementation .............................................................. 56

3.4.2 Neurovascular Responses to Mental Stress Following Omega-3

Supplementation....

4. Chapter 4

61

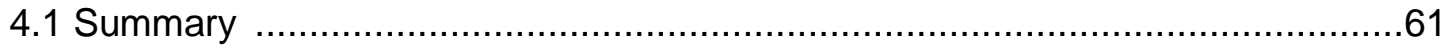

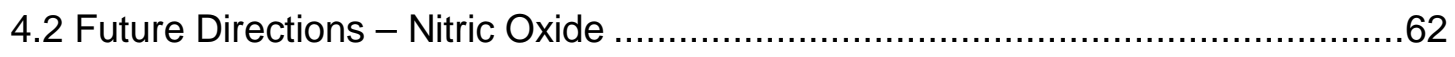

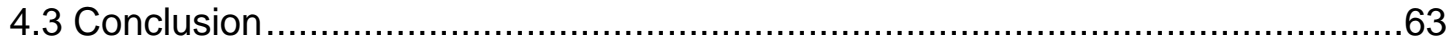

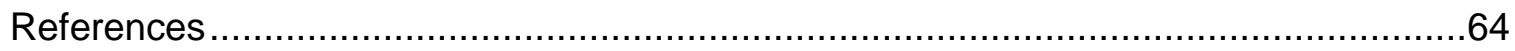

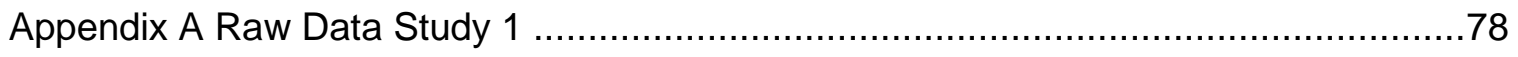

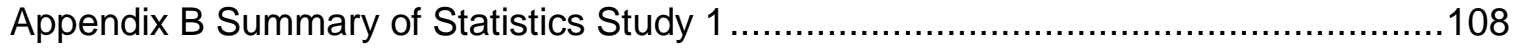

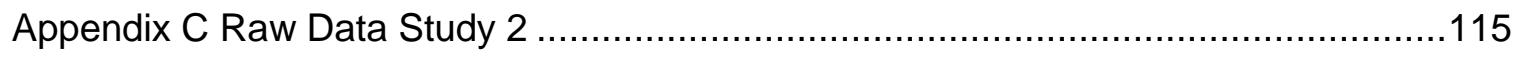

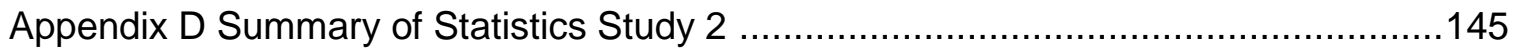

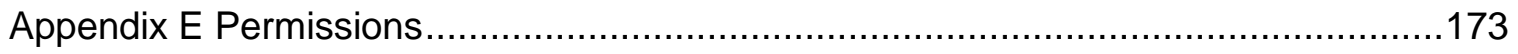




\section{List of Figures}

Figure 1.1 A representation of two cardiac cycles .............................................

Figure 1.2 A representative neurogram and corresponding blood pressure .................8

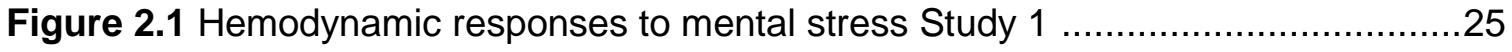

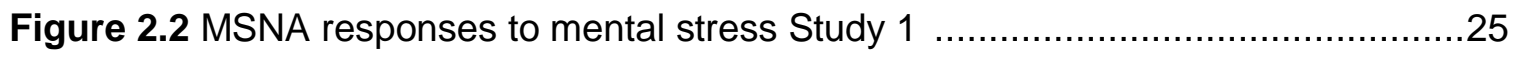

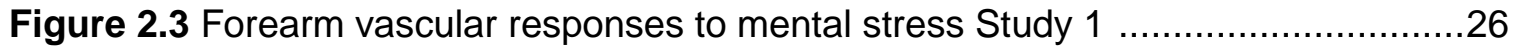

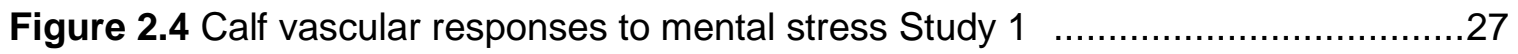

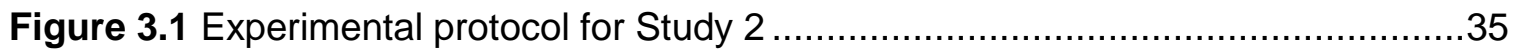

Figure 3.2 Resting MSNA responses in PHT and NT Study 2 ............................42

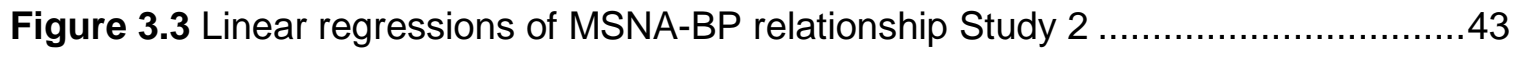

Figure 3.4 Linear regressions of MSNA-HR relationship Study $2 \ldots \ldots \ldots \ldots \ldots \ldots \ldots \ldots . . \ldots \ldots$

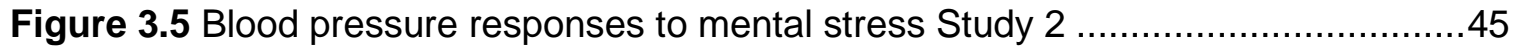

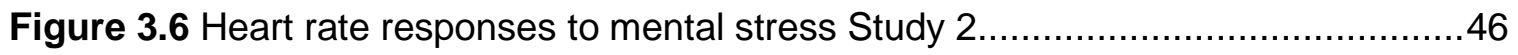

Figure 3.7 Forearm vascular responses to mental stress Study 2 .........................49

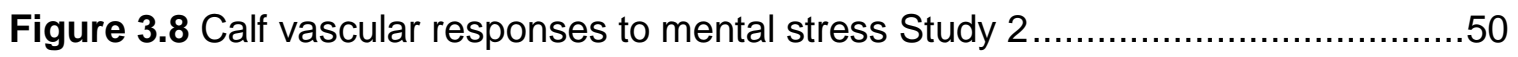

Figure 3.9 Blood pressure responses to mental stress in combined

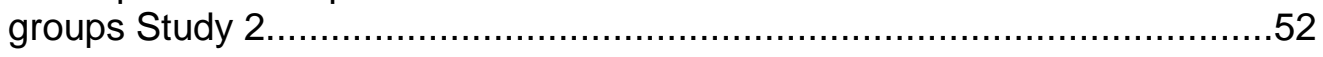

Figure 3.10 MSNA responses to mental stress in combined groups Study $2 \ldots \ldots \ldots \ldots . . .53$

Figure 3.11 Total MSNA responses to mental stress in combined groups Study 2

Figure 3.12 Forearm vascular responses to mental stress in combined groups Study 2

Figure 3.13 Calf vascular responses to mental stress in combined groups Study 2 


\section{List of Tables}

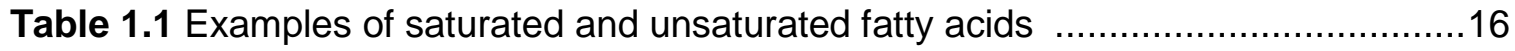

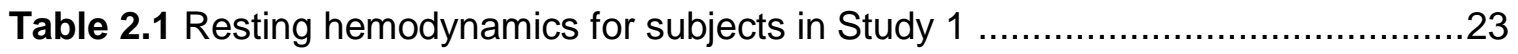

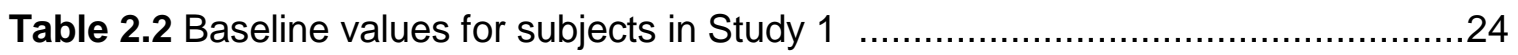

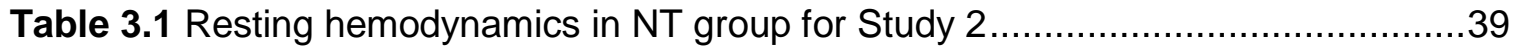

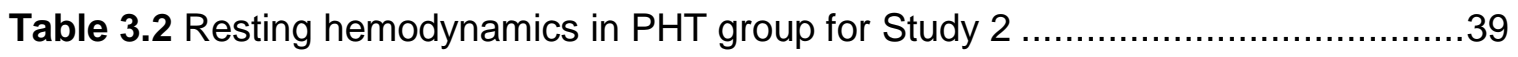

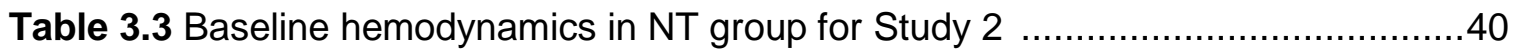

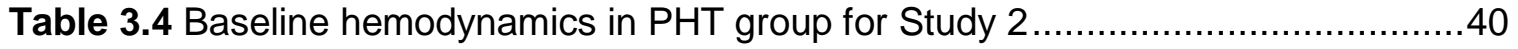

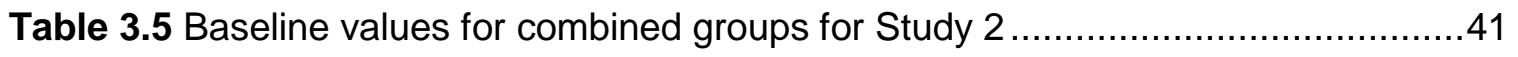

Table 3.6 Changes in MSNA during mental stress in normotensives Study $2 \ldots \ldots \ldots \ldots . . .47$

Table 3.7 Changes in MSNA during mental stress in prehypertensives Study $2 \ldots \ldots \ldots \ldots . . .48$

Tables A.1 - A.30 Tables of raw data from Study 1 for all subjects ....................78-107

Tables B.1 - B.31 Tables of statistical analyses from Study 1 for all subjects......108-114

Tables C.1 - C.28 Tables of raw data from Study 2 for all subjects ..................115-144

Tables D.1 - D.100 Tables of statistical analyses from Study 2 for all subjects....145-172 


\section{Preface}

The article presented in chapter 2 of this thesis is used with permission from the American Physiological Society, as a collaborative work with co-authors Dr. John Durocher, Dr. Jason Carter. All data collection was conducted in the laboratory of Dr. Carter at Michigan Technological University. My personal contribution to the article included recruitment, orientation, conduction, data analysis and statistical analysis of the study. I had a significant role in the authorship of the Introduction, Methods, and Results sections, with a smaller but significant contribution to the Discussion section. 


\section{Acknowledgements}

This dissertation is a testament not only to perseverance and hard work, but also to the constant support, advice and assistance I have received from many people along the way. First and foremost, I must thank my Lord Jesus Christ for the grace he has bestowed upon me. Through thick and thin, He is my rock. Second, I deeply thank and appreciate the mentorship and guidance I have received from my advisor, Dr. Jason Carter. The patience he has shown me through particularly through the process of composing this thesis, but also during my time working as his research assistant, was second to none. I will continue carry on the skills and knowledge he has passed down to me wherever my career takes me. Third, I thank my committee members for all their patience and guidance.

I would also like to thank my family; Dad, Mom, Becky, Joe, Eric, David, Johanna, Emily, Andrew, Jacob, Mary, Elsa, Carrie, all my brothers/sisters-in-law, and my nieces and nephews (especially my nieces and nephews) for your never-ceasing love and support. The Lord is my rock, but you all are the ground on top of that rock.

Thank you to my friends for all your advice and encouragement. Thank you to Rick for always being there, supporting me, and putting up with me during our endless hours of training. A special thanks goes to Agustin, Lilia and Leila, as well as Israel, Judy, Manu, Micah and Ty, and Matt, Heather, Kaisa, Ava and Lewis. I simply would not be the same person I am today without you. Thank you to Sikhu for always lending an ear, and helping me push through to the end. Thank you to Louisa and Jesse for letting me stay with them over the summer, and always providing awesome training days. Stephanie, G, Raman, Tejal, Jim, Andi, Mark, Lauren, Rob, Shannon, Joel, Valerie, Sinan, Ana, Corey, Rachel, and all my friends, thank you for being awesome!

I wish to thank my friends and fellow students in our lab. I don't know how I could repay you all for the help you have given me in completing this thesis. John Durocher, John Lawrence, Jenna Klein, Sarah Stream, Huan Yang, Robert Larson, Kristen Reed, Michelle King, Ashley Yenior, and Jen Witting, thank you for all the help, particularly over the last few months. I wish every graduate student could have the support that you all gave to me. Finally, to Terry Anderson, a deep thank you for everything you have done, and for making this time as easy as possible. I appreciate you! 


\section{List of Abbreviations}

\begin{tabular}{ll} 
ALA. & Alpha-linolenic acid \\
BP. & Blood pressure \\
CBF. & Calf blood flow \\
CVD. & Cardiovascular disease \\
CVR. & Calf vascular resistance \\
CVC. & Calf vascular conductance \\
DAP. & Diastolic arterial pressure \\
DHA. & Docosahexaenoic Acid \\
EPA. & Eicosapentaenoic Acid \\
FBF. & Forearm blood flow \\
FVR. & Forearm vascular resistance \\
FVC. & Forearm vascular conductance \\
HF. & High frequency component of heart rate variability \\
HR. & Heart rate \\
HRV. & Heart rate variability \\
LF. & Low frequency component of heart rate variability \\
MAP. & Mean arterial pressure \\
MSNA. & Muscle sympathetic nerve activity \\
NE. & Norepinephrine \\
NT. & Normotension \\
NO. & Nitric Oxide \\
PHT. & Prehypertension \\
PUFA. & Polyunsaturated fatty Acid \\
RRI. & R-R Interval \\
SAP. & Systolic arterial pressure \\
\hline
\end{tabular}




\section{Abstract}

Hypertension is the most prevalent form of cardiovascular disease (CVD) in the world, and is known to increase the risk for developing other diseases. Recently, the American Heart Association introduced a new classification of blood pressure, prehypertension (PHT). The criteria for PHT include a systolic of $120-139 \mathrm{mmHg}$ and/or a diastolic blood pressure of $80-89 \mathrm{mmHg}$. It has been observed that individuals with $\mathrm{PHT}$ have a higher risk of developing hypertension later in life. Therefore, it is important to understand the mechanisms contributing to PHT in order to possibly prevent hypertension. Omega-3 fatty acids found in fish oils have been suggested as a means of lowering blood pressure. However, little is known on the effects of fish oil in PHT humans. Therefore we conducted two studies. In Study 1 we investigated PHT and normotensive (NT) individuals during a mental stress task. Mental stress is known to contribute to the development of hypertension. In Study 2 PHT and NT subjects were placed in an eight week double-blind placebo controlled study in which subjects consumed $9 \mathrm{~g} /$ day of either fish oil or placebo (olive oil) in addition to their regular diets. Subjects were tested during a resting baseline (seated and supine), 5 minutes of a mental stress task, and 5 minutes of recovery both pre and post supplementation. We measured arterial pressure (AP), heart rate (HR), muscle sympathetic nerve activity (MSNA), and forearm and calf vascular responses. In Study 1 PHT demonstrated augmented AP and blunted vasodilation during mental stress, but MSNA did not change. In Study 2, fish oil did not directly influence blood pressure, MSNA or vascular responses to mental stress. However, it became clear that fish oil had an effect on some but not all subjects (both PHT and NT). Specifically, subjects who experienced a reduced blood pressure response to fish oil also demonstrated a decrease in MSNA and HR during mental stress. Collectively, the investigations in this dissertation had several novel findings. First, PHT individuals demonstrate an augmented pressor and blunted vascular response to mental stress, a response that may be contributing to the development of hypertension. Second, fish oil does not consistently lower resting blood pressure, but the interindividual responses may be related to MSNA. Third, fish oil attenuated the heart rate and MSNA responses and to mental stress in both PHT and NT. In conclusion, we found that there are both similarities and differences in the way PHT and NT individuals respond to mental stress and fish oil. 


\section{Chapter 1}

\section{Literature Review}

\subsection{Hypertension}

Over 80 million Americans are diagnosed with at least one form of cardiovascular disease (CVD). The most prevalent form of CVD is hypertension, affecting 76.4 million Americans and as many as 1 billion people worldwide (Chobanian et al., 2003). Cardiovascular related mortality, such as ischemic heart disease and stroke, increase dramatically when a comorbidity of high blood pressure is present. Additionally, 7.1 million people die from hypertension related causes annually (Brundtland, 2002; Chobanian et al., 2003).

The concept that the blood circulates in the body was first suggested in the early 1600 s by William Harvey (Harvey, 1628). At the time this was a giant step forward in the understanding of the human body and how it functions. Nearly 100 years later the first measurements of blood pressure were taken by Stephen Hales (Booth, 1977), who measured blood pressure from an artery of a horse using a nine-foot glass tube. The technique of analyzing blood pressure was later refined and modified by Jean Poiseuille (1828) and Carl Ludwig (1847) to essentially the method we use today (Norris, 1917). However, even before measurements of arterial pressure were conducted there seemed to be an understanding of a type of "hard pulse disease," which we now know as hypertension (Esunge, 1991).

The classification of hypertension is considered to be a systolic arterial blood pressure $\geq 140 \mathrm{mmHg}$ and/or a diastolic arterial blood pressure $\geq 90 \mathrm{mmHg}$ (Chobanian et al., 2003). Keith et al. (1974) found that there was a direct relationship with increased blood pressure and incidence of cardiovascular disease. Furthermore, risk of developing cardiovascular disease increases 2-fold when systolic arterial blood pressure is $130-139$ $\mathrm{mmHg}$ and/or diastolic arterial blood pressure is $85-89 \mathrm{mmHg}$ (Vasan et al., 2001). These findings initiated the introduction of a new classification known as prehypertension. Having a systolic blood pressure of $120-139 \mathrm{mmHg}$ and/or diastolic blood pressure of $80-89 \mathrm{mmHg}$ is now classified as "prehypertensive." Prehypertension is associated with an increased risk of developing hypertension later on in life (Vasan et al., 2001; Moreira et al., 2008). The reasons for this increased risk remain unclear. 
Arterial pressure is closely regulated by the nervous system. Control of whole body arterial blood pressure is done through a negative feedback mechanism known as the arterial baroreflex. Modified stretch receptors located in the aortic arch, carotid sinuses and the cardiopulmonary blood vessels detect elastic changes in the arterial wall. Once a stretch is detected the baroreceptors send afferent nerve signals back to the area of the brain known as the medulla, more specifically the nucleus tractus solitarus. A reflex mechanism is then triggered sending efferent sympathetic and parasympathetic nerve signals down to the cardiovascular system to either increase or decrease blood pressure, respectively.

It has been recognized that arterial baroreceptors reset during hypertension. This effect is seen in studies comparing arterial blood pressure oscillations in normotensive and hypertensive subjects. Both groups responded similarly to vasoactive drugs, with the hypertensive group operating at a higher blood pressure compared to normotensive controls (Wallin et al., 1973). Mechanisms behind the resetting of the baroreflex remain in question, but overactive sympathetic nerve activity remains a primary suspect.

With the introduction of new techniques to test sympathetic nerve activity (i.e. microneurography and norepinephrine (NE) spillover) studies of borderline and mild hypertensive individuals suggest that augmented sympathetic nerve activity contributed to hypertension (FitzGerald et al., 1981; Anderson et al., 1989). Urine and plasma NE have also been shown to be elevated in established hypertension (Esler \& Nestel, 1973; Goldstein, 1983). Collectively, these findings provided important insight into the differences between hypertension and normal healthy humans. Similar discrepancies began to appear between these populations during physiological and psychological stimuli.

Sympathetic nerve activity increases during a mental challenge in most individuals (Anderson et al., 1987; Tidgren \& Hjemdahl, 1989; Wallin et al., 1992; Carter et al., 2005; Carter \& Ray, 2009), and the concurrent increase in blood pressure and nerve activity does not follow the classic inverse relationship between sympathetic vasoconstrictor activity and arterial pressure seen at rest (Wallin et al., 1973; Durocher et al., 2011). This altered neurovascular responses to stress has been suggested as a possible mechanism contributing to the development of hypertension (Esler et al., 2008). 
Therefore, understanding how to assess autonomic nerve activity, and how neural and cardiovascular respond to mental stress is clinically relevant.

\subsection{Measuring Autonomic Nerve Activity}

\subsubsection{Hemodynamic Measurements}

Perhaps the oldest and most commonly used assessment of sympathetic and parasympathetic activity is done by examining changes in hemodynamic measurements (i.e. arterial pressure and heart rate). The autonomic nervous system innervates the heart and the blood vessels affecting both heart rate and blood pressure. During physiological and psychological stress, sympathetic nerves increase heart rate and blood pressure (Barcroft, 1946). For example, during exercise sympathetic nerve activation causes heart rate to increase and blood vessels in non-active areas (i.e., gut, kidneys, etc.) to constrict resulting in an increase in blood pressure (Rushmer et al., 1959). Sympathetic nerves innervate the heart causing an increase in heart rate (measured in beats per minute), stroke volume (i.e. the amount of blood per minute), and cardiac output (i.e. the amount of blood that exits the heart per minute). Constriction of the vascular wall increases total peripheral resistance which increases blood pressure (Shepherd, 1987). Arterial pressure is a measure of the relationship between cardiac output and total peripheral resistance, the equation of which is shown below (Heymans, 1958):

Mean Arterial Pressure $=$ Cardiac Output $\mathrm{x}$ Total Peripheral Resistance

This direct relationship allows for analysis of changes in pressure as direct changes in the cardiovascular system, which in turn infers changes in autonomic control. In such a way, an increase in heart rate and blood pressure is an indication of sympathetic nerve stimulation and parasympathetic withdrawal, and likewise a decrease represents parasympathetic activation and sympathetic withdrawal. Therefore, monitoring changes in hemodynamic measurements gives us an indirect, but informative, method of analyzing autonomic function.

There are several positive aspects of hemodynamic assessment of autonomic nerve activity. These measurements are non-invasive and easy to conduct. Heart rate is 
easily measured using electrocardiography, and requires relatively inexpensive equipment to acquire and analyze. Similarly, blood pressure can be measured using a simple, inexpensive sphygmomanometer. Modern methods for recording these variables can now be done virtually anywhere, including remotely so subjects do not have to be confined to a laboratory setting, and are free to move about while their hemodynamics are being monitored continuously. However, there are limitations to these measurements. One limitation is that there is a high amount of variability between subjects. Hemodynamics are influenced by many factors (i.e. activity, time of day, anxiety, etc.), and reproducibility is not always accurate because of these conditions (Parati et al., 1988). Another limitation is that hemodynamic are indirect measurements of autonomic acitivity, and subject to the methods used to stimulate them. These limitations make it difficult to support hemodynamic measurements as a sole measure of autonomic activity. However, taken in combination with other measurements, hemodynamics can provide an important picture of whole body peripheral nerve function.

\subsubsection{Heart Rate Variability - Spectral Analysis}

Heart rate, as stated above, is an important hemodynamic measurement that indirectly depicts autonomic nerve function. The sympathetic and parasympathetic branches of the peripheral nervous system innervate the heart, thus affecting the increase and decrease of heart rate, respectively. This reciprocal relationship between sympathetic and parasympathetic branches is known as sympathovagal balance (Malliani et al., 1991). Early analysis of heart rate variability looked primarily at the individual variability between resting heart rate measurements (Malmo \& Shagass, 1949). However, more recent analyses of the neural control of heart rate has been made possible with more advanced computer and mathematic capabilities using a method known as power spectral analysis of heart rate variability. 


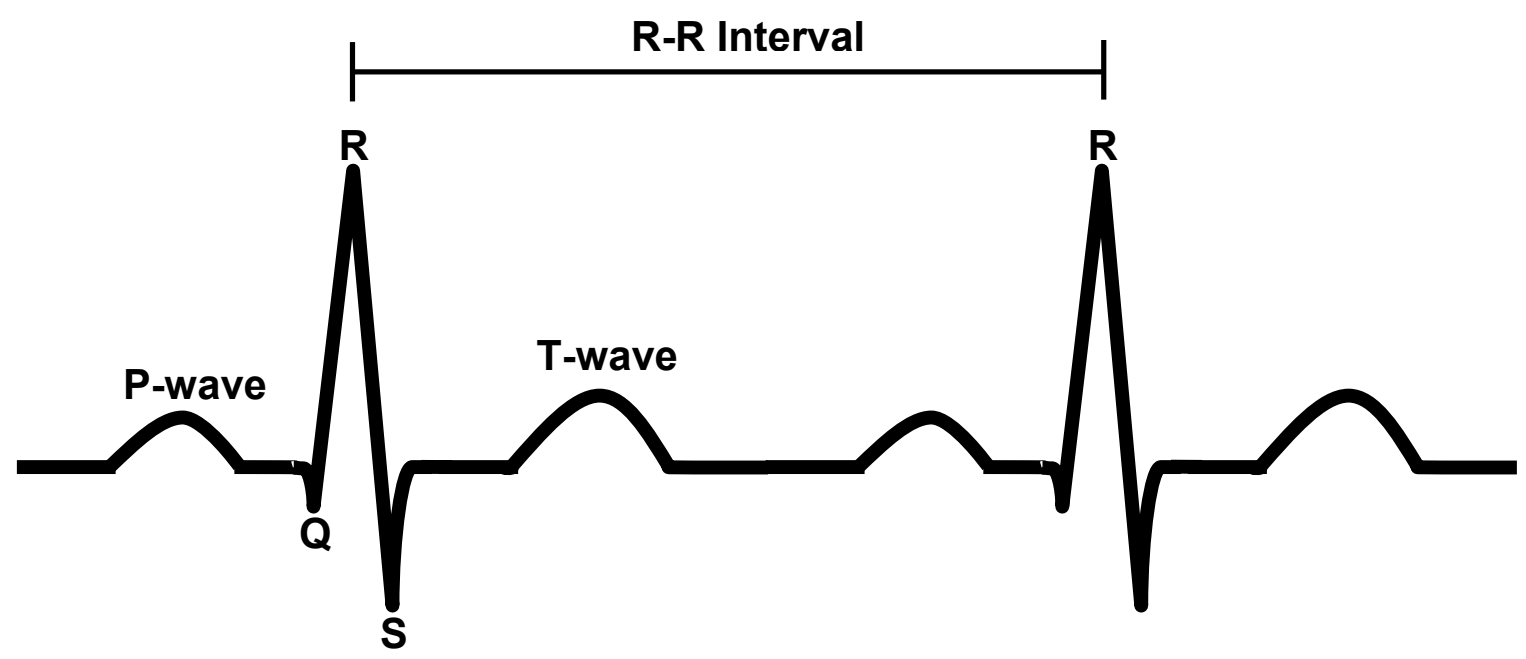

Figure 1.1. A representation of two cardiac cycles as seen in a typical electrocardiogram recording. The P-wave represents atrial depolarization, the QRS complex represents ventricular depolarization, and the T-wave represents ventricular repolarization. The time-distance, in milliseconds, between consecutive R-waves is known as the R-R Interval (RRI).

This technique utilizes mathematical equations to analyze changes in the $R-R$ intervals (RRI) of each cardiac cycle. A representative model of two cardiac cycles is depicted in figure 1.1.Analysis of oscillations in RRIs throughout the time series can be reported as frequency and amplitude, and are compared to determine strength of autonomic activity (Pagani et al., 1986; Malliani et al., 1991). For example, an increase in the RRI would suggest influence from the vagus (parasympathetic) nerve, and a decrease in RRI would signify sympathetic control over heart rate. High frequency (HF) oscillations $(\sim 0.25 \mathrm{~Hz})$ are representative of primarily parasympathetic nerve activity from the vagus nerve. Low frequency (LF) oscillations $(\sim 0.1 \mathrm{~Hz})$ demonstrate both sympathetic and parasympathetic activity (Pomeranz et al., 1985; Pagani et al., 1986). The relationship between both peripheral nervous system branches on heart rate control is further represented by the ratio between the two frequencies, known as the LF/HF ratio (Pagani et al., 1986). Power spectral analysis can serve as an important tool in interpreting autonomic control of cardiovascular function.

There are several limitation associated with heart rate variability. Similar to standard hemodynamics, power spectral analysis is bound to multiple external factors affecting heart rate and blood pressure. Additionally, there is speculation as to the accuracy of the use of computational analysis as a means of measuring autonomic 
activity instead of direct recordings (Eckberg, 1997). Nevertheless, power spectral analysis of heart rate variability has given us valuable insight into the neural mechanisms contributing to cardiovascular function, and when used in conjunction with other techniques can provide a more complete measure of neural-cardiovascular interactions.

\subsubsection{Norepinephrine - Urine and Plasma}

Measurements of catecholamine levels in blood (i.e. plasma NE and epinephrine) and urine are commonly used to assess sympathetic activity. Early research in cats demonstrated a correlation with sympathetic nerve firing and NE release into the blood (Brown \& Gillespie, 1957). Engelman et al. (1970) performed an enzymatic assay to analyze catecholamines in human plasma and urine, and were able to isolate circulating norepinephrine. The technique provided a more specific method of assessing sympathetic activity. Sympathetic nerves primarily excrete NE as their neurotransmitter. Molecules exit the pre-synaptic neuron and enter the synaptic space where NE can either bind to the post-synaptic neuron, be metabolized by catechol-Omethyltransferase, be absorbed into the circulation, or be taken back into the presynaptic neuron (Hertting, 1964). Any amount of NE not used or recycled is dissipated and taken up into the blood stream, and further filtered by the kidneys into the urine. It is in these two areas (plasma and urine) that NE can provide a measure of whole body sympathetic activity (Hjemdahl, 1984). However, the use of plasma or blood samples only provides a small, singular measurement of overall activity. Additionally, a high level of plasma NE does not always indicate a high level of nerve activity (Goldstein 2003). Acute sympathetic measurements are not measurable with this method, and the amount of NE absorbed into the blood is a small fraction (5-10\%) of total neurotransmitter excreted at the nerve synapses (Grassi \& Esler, 1999). Though plasma and urine NE measurements give us an idea of whole body sympathetic activation, the picture is not complete, and does not provide a means of continual measurement of neural activity.

\subsubsection{Norepinephrine - Regional Spillover}

The limitations that plasma and urine NE measurements pose brought about the introduction and implementation of a technique for measuring plasma NE kinetics, also 
known as NE spillover rate (Esler et al., 1989). This process uses the infusion of radioactive-labeled NE to measure the appearance rate of NE in the plasma. The spillover is acquired by measuring the relationship between the radio-labeled NE infusion rate and the appearance of radioactive NE in the plasma (Esler et al., 1979; Esler et al., 1989). The benefit of NE spillover compared to a simple plasma sampling is that through use of arterial or venous catheter, plasma uptake of NE can be measured in real-time at specific regions not otherwise accessible (i.e. cardiac and renal regions) (Esler et al., 1984a). The measurement of NE kinetics is currently the only technique used in humans that can measure activity in deep, region specific tissue beds. However, NE spillover has its drawbacks. Spillover measures overall sympathetic outflow via NE measurements, but the exact source of the NE is unknown. Sympathetic nerves release the neurotransmitter NE, but this same molecule is released by the adrenal gland as well, and the spillover technique is not able to determine the source of NE (Esler et al., 1984a). Furthermore, this technique is not a direct measurement of sympathetic nerve activity. Although there are drawbacks and it is only used in a few laboratories throughout the world, NE spillover is recognized as a robust and superior method of measuring sympathetic tone.

\subsubsection{Microneurography}

A major advancement in our ability to measure sympathetic nerve activity occurred in the late 1960s. Direct peripheral muscle nerves were successfully recorded in humans by using a tungsten needle with a microelectrode at the tip, and inserted into a nerve fascicle to obtain multi-unit discharge of activity (Hagbarth \& Vallbo, 1967; Vallbo \& Hagbarth, 1967; Hagbarth \& Vallbo, 1969). This technique, termed microneurography, was refined and found to be a very useful tool in measuring sympathetic nerve activity (Figure 1.2) (Delius et al., 1973). Moreover, microneurography is currently the only direct method of measuring sympathetic nerve activity in humans (Grassi \& Esler, 1999). The microneurographic technique measures post-ganglionic multi-fiber efferent sympathetic nerve activity to skeletal muscle beds, referred to as muscle sympathetic nerve activity (MSNA) (Vallbo et al., 2004). MSNA is typically measured as integrated bursts of activity per minute. MSNA bursts are coupled to the cardiac cycle, and if a burst of activity is going to occur, it will do so in one cardiac cycle 
(Delius et al., 1972). Therefore, reporting MSNA as bursts per 100 heartbeats is also an accepted quantification. Total MSNA activity is another measurement commonly reported. Total MSNA takes into account not only the burst activity per minute but also the amplitude and area underneath each integrated burst (Sundlof \& Wallin, 1978). Because MSNA is most commonly measured as multifiber nerve recordings, multiple integrated sympathetic firings demonstrate varying amplitudes of firing strength. To account for this, analysis of total activity is employed using a baseline normalization procedure.

Microneurography has several strong attributes. First, it is currently the only direct method of assessing sympathetic neural activity in humans. Second it correlates strongly with other measures of autonomic activity such as the NE spillover technique (Wallin et al., 1981). Another advantage is that it allows for continuous measurement of

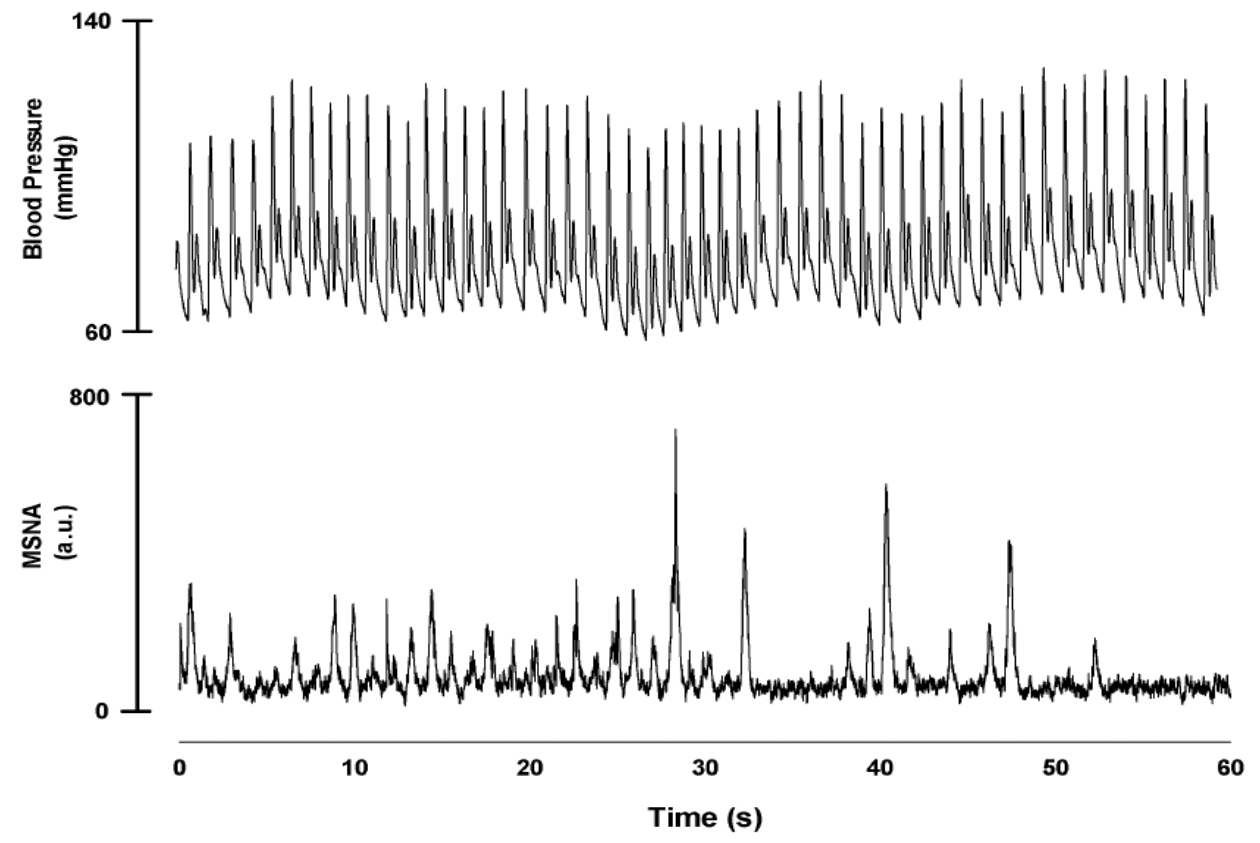

Figure 1.2. A representative neurogram and the corresponding blood pressure for one minute of baseline. Notice the relatively close relationship between oscillations in blood pressure and nerve activity. MSNA, muscle sympathetic nerve activity; a.u., arbitrary units 
activity during different interventions. Techniques such as plasma/urine NE measurements only take isolated samples, thus providing a non-continuous measurement of sympathetic activity. However, there are disadvantages associated with microneurography. The technique is mildly invasive and can only be done by trained individuals. It is highly variable between individuals, though it remains highly reproducible when tested over a period of time, even up to years later (Sundlof \& Wallin, 1977). Another disadvantage is that the technique is sensitive to movement, and the electrode can become dislodged easily. Therefore, it is only possible to use microneurography in a laboratory setting, with subjects typically in the supine or seated positions. Despite these limitations, microneurography, along with norepinephrine spillover, remains the "gold-standard" for assessing sympathetic nerve activity.

\subsection{Neural Responses to Mental Stress}

The advancement in our ability to measure autonomic nerve activity has allowed us to further investigate the effect of psychological stress in humans. Mental stress consistently increases heart rate, blood pressure and forearm blood flow, which are closely linked with the sympathetic and parasympathetic nervous systems (Blair et al., 1959; Barcroft et al., 1960). Simultaneous neuroendocrine responses can also be observed during this cardiovascular response to mental stress (Hjemdahl et al., 1984). Ultimately, markers for established psychological distress were identified as an increase in heart rate and force contraction, skeletal muscle vasodilation, and increased circulating catecholamines (Herd, 1991).

Epinephrine is strongly linked to the mental stress response. When compared to non-psychological stimuli, such as the cold pressor test, mental stress causes a greater increase in heart rate, skeletal muscle vasodilation and increased circulating epinephrine. This is compared to no increase in epinephrine during a physical stressor like the cold pressor test (LeBlanc et al., 1979). Notably, norepinephrine levels were greater during cold pressor test compared to mental stress. Because norepinephrine is closely linked with the sympathetic nervous system, these findings provided early evidence that a combination of circulating catecholamine and sympathetic nerve responses were causing the physiological changes during mental stress. 
Circulating plasma norepinephrine has been closely linked with sympathetic nerve activity (Wallin, 1984). Today, the most common means of measuring sympathetic nerve activity include plasma or urine norepinephrine sampling, radioactive labeled norepinephrine spillover from cardiac and renal sympathetic beds, and microneurographic measurements of MSNA from the peroneal nerve. Plasma norepinephrine measurements have been shown to increase during mental stress (Dimsdale \& Moss, 1980; Jones et al., 1996). However, in one study mental stress increased plasma norepinephrine in older, but not young, subjects ( $\mathrm{Ng}$ et al., 1994). Norepinephrine spillover has also been shown to increase during a mental challenge (Goldstein et al., 1987; Lindqvist et al., 1993; Wilkinson et al., 1998). MSNA recordings during mental stress typically demonstrate increases in sympathetic nerve activity (Anderson et al., 1987; Hjemdahl et al., 1989; Wallin et al., 1992; Carter et al., 2005; Carter \& Ray, 2009), but recent work highlights substantial variability regarding the MSNA reponses to mental stress (Carter \& Ray, 2009).

\subsection{Cardiovascular Responses to Mental Stress}

The reaction of the body to stress is a compensatory mechanism. The human body is placed under stress constantly throughout any given day. Psychological and emotional stressors, in particular, are known to place a great amount of strain on both mental and physiological parameters in the human body. In "The Expression of the Emotions in Man and Animals" Charles Darwin describes a general state of expression (Darwin, 1886). The common response in animals is an activated nervous system, muscle trembles, a combination of emotions such as rage, joy and terror. Further observation of fear and astonishment resulted in multiple changes to a biological system, including increases in heart rate, pale skin, muscle trembles, widely open eyes and mouth (Darwin, 1886). Years later, Walter Cannon would describe these same responses as an "emotional experience" (Cannon, 1914). Additionally, Cannon and others discovered specific physiological changes that occur during emotional stimuli, most notably the release of adrenaline (i.e. epinephrine) from the adrenal glands (Newton, 1930). He also noted a specific interaction with the physiological system and "sympathin" (Cannon, 1933). We now refer to sympathin as noradrenaline (i.e. norepinephrine), the main neurotransmitter released from sympathetic neurons. This 
hormone response causes a response similar to that directed by nervous system, which includes pupil dilation, blood vessel constriction and rapid heart rate (Cannon, 1914).

Cannon introduced the term "fight or flight" to describe the sympathetic nervous system's reaction to stressors (Cannon, 1927). These findings introduced an important idea to our current views of multiple physiological systems' responses to emotional stress. The autonomic nervous system is comprised of the parasympathetic and sympathetic nervous systems, and is a primary controller of the cardiovascular system. However, as Cannon and others discovered, there is an interaction in adrenergic activity from the nervous and endocrine systems (Cannon, 1933). More specifically, the autonomic nervous system and adrenal (medulla and cortex) responses work in parallel. However, Hans Selye is widely credited with highlighting a clinical reason for studying neuroendocrine responses to psychological stress.

The "General Adaptation Syndrome" was first proposed by Hans Selye (Selye, 1936). Selye discovered, by accident, that psychological stress to rats resulted in specific physiological responses, specifically an enlargement of the adrenal glands and increased ulcers. This sparked a whole new line of "stress" research. Investigations into the general response to different stressors such as cold, injury, exercise, drugs and others were conducted producing what Selye termed the general adaptation syndrome. Responses to many different types of stress results in "systemic damage", and originate in the hypothalamus which can influence processes throughout the body including the adrenal glands, heart, and vascular system (Selye \& Fortier, 1950).

In years to follow, Brod et al. (1959) reported that a mental stress task such as mental arithmetic caused similar physiological response as emotional stress or fear. Notably heart rate and blood pressure increased, and interestingly blood flow increased to peripheral muscles and decreased in the renal vascular bed. Forearm vasodilation during mental stress has been observed repeatedly in multiple studies (Barcroft \& Edholm, 1945; Blair et al., 1959; Rusch et al., 1981; Dietz et al., 1994; Carter et al., 2005) and has led to the description of a classic "defense reaction" (Herd, 1991).

Peripheral blood flow in forearm and calf muscular beds are common measurements during a mental challenge. When blood flow to the peripheral muscles was studied during post-hemorrhagic fainting, there was a marked vasodilation at which the point when faint occurred (Barcroft \& Edholm, 1945). Interestingly, it has been 
observed that fainting also occurs during emotional distress, and studies began to incorporate mental and emotional stress as a means of analyzing cardiovascular responses during faint (Roddie, 1977).

Forearm vasodilation consistently increases during mental and emotional stress (Blair et al., 1959; Barcroft et al., 1960; Rusch et al., 1981; Dietz et al., 1994; Kuipers et al., 2008). Calf blood flow has been found to increase in some studies (Blair et al., 1959; Kuipers et al., 2008), but not all (Rusch et al., 1981). The proposed mechanisms contributing to vasodilation in the limbs include sympathetic mediated adrenergic responses, nitric oxide (NO) release from the endothelium, and circulating catecholamines.

The response to mental and emotional stress observed in these investigations fits with Cannon's "fight or flight" description. However, it has become clear that mental stress reactivity is more complicated than simply preparing the body for fight or flight. Observing and analyzing this stress response has become crucial to our understanding of how mental and emotional stress contributes to the risk of cardiovascular disease. With the improvements in technology it has become easier to measure changes in heart rate, blood flow, and autonomic nerve activity. This has been crucial in our understanding of the how neurological and humoral responses to stress influence health in humans.

\subsection{Mental Stress and Cardiovascular Disease}

It has been noted that an acute mental stress can adversely affect cardiovascular health. One example of this is seen in the increased number of cardiac related deaths following a traumatic event such as a major earthquake (Leor et al., 1996). Additionally, electrocardiogram recordings during an earthquake event have shown that heart rate significantly increased in patients. Spectral analysis of this response suggested an increased sympathetic nerve response and reduced parasympathetic tone (Huang et al., 2001). Furthermore, mental and emotional stress are closely linked with cardiovascular diseases such as myocardial ischemia (Deanfield et al., 1984), endothelial dysfunction (Spieker et al., 2002), atherosclerosis (Lynch et al., 1997) and hypertension (Esler et al., 2003). Perhaps the most well known study to linking stress and risk for cardiovascular disease was performed by Timio et al. (1988). They found that cloistered nuns, secluded 
from society and living a very controlled and non-stressful lifestyle, did not experience the typical rise in blood pressure often seen with increasing age (Timio et al., 1988). Further, it has been suggested that chronic mental stress leads to a rise in blood pressure over time (Esler et al., 2008). The mechanisms by which mental stress affects cardiovascular disease are not fully understood, but a clear link exists, particularly in hypertension.

\subsection{Treatment of Hypertension}

Hypertension has been linked to a number of cardiovascular-related deaths. Therefore, early treatment and/or prevention of high blood pressure has become a primary focus in clinical care. Treating hypertension through pharmacological or nonpharmacological methods can reduce the risk of stroke, myocardial infarction, and heart failure (Neal et al., 2000). Current treatment of hypertension is primarily focused on pharmacological intervention. Common types of anti-hypertensive medications include diuretics, ACE inhibitors, $\alpha$ and $\beta$-blockers and calcium channel blockers (Epstein, 2010). Most patients require at least two medications to lower their blood pressure (Cushman et al., 2002; Epstein, 2010). It is evident that cost of treatment for hypertension can escalate quickly. The American Heart Association recently projected that the cost of hypertension treatment will increase from $\$ 130.4$ billion in 2008 to $\$ 200.3$ billion in 2030 (Heidenreich et al., 2011). Therefore, it is prudent to investigate alternate methods of treatment and prevention of high blood pressure.

The most recent joint report on hypertension currently lists lifestyle modifications as the only non-pharmacological treatment plan (Chobanian et al., 2003). Lifestyle modifications include weight loss, diet and exercise. The effects of omega-3 fatty acids, found in fatty fish or extracted from certain plants, have intrigued many patients and clinicians as a potential non-pharmacological approach to blood pressure control.

Supplementation with omega-3 fatty acids has been shown to reduce blood pressure in hypertensive but not normotensive individuals (Appel et al., 1993; Morris et al., 1993). There is also some debate as to the dosage needed to observe a blood pressure lowering effect. Studies have found that doses are only effective in very high amounts, such as $15 \mathrm{~g} /$ day (Knapp \& FitzGerald, 1989). Other studies suggest that moderate amounts are capable of lowering blood pressure in those with mild- 
hypertension (Appel et al., 1993; Morris et al., 1993). Additionally, Howe et al. (1991) found that fish oil was most beneficial in lowering blood pressure when combined with a low sodium diet in spontaneously hypertensive rats. It is suggested that when combined with other non-pharmaceutical methods, such as lifestyle and dietary modifications, omega-3 fatty acid supplementation may be beneficial in lowering blood pressure, particularly in mildly hypertensive individuals (Howe, 1995). Prevention of hypertension through omega-3 fatty acid supplementation, particularly in prehypertensive individuals who are at higher risk for development of hypertension, seems possible.

\subsection{Polyunsaturated Fatty Acids}

Throughout history there have been home remedies that claim to be cure-all drugs. These non-pharmaceutical and "natural" medicines have always been popular among individuals and main-stream media. Some of these medicinal tools have proven useless and sometimes dangerous, yet others have been effective. In particular, dietary modifications have been viewed as an obvious measure in disease prevention. Certain populations throughout the world have strikingly lower rates of cardiovascular disease (CVD) than in the United States or Western Europe, and diet is believed to contribute heavily to these differences. One such group that has shown remarkably low incidence of CVD is the Inuit Culture in Alaska and Greenland (Bang et al., 1971). Analysis of the Inuit diet revealed a much higher consumption of polyunsaturated fatty acids (PUFAs), specifically from marine oils, when compared to their Danish counterparts (Bang et al., 1980). These findings led to both interest and research on fish oil, and its effect on health and cardiovascular risk.

Recently, public awareness of PUFA health benefits has dramatically increased. Since the studies conducted by Bang and colleagues (1971 \& 1980), the idea that fish oil could influence CVD has grown considerably. However, the mechanisms by which these "healthy" fats influence human physiology remain unclear. There are many suggested benefits of increasing PUFA consumption into the American diet, but one benefit, lowering blood pressure, seems to be at the forefront of public opinion.

It is well-documented that unsaturated fats have more health benefits when compared to saturated fatty acids. The difference between PUFA's and saturated fatty acids is found in their molecular structure. Saturated fatty acids contain single bonds 
between carbon molecules. Unsaturated fats are organic molecules containing one or more double bond in the general fatty acid structure; therefore, PUFAs are unsaturated fats with two or more double bonds. Table 1.1 shows a list of common saturated and unsaturated fats.

The most common PUFAs in a typical diet are omega-3 and omega- 6 fatty acids. Omega-3 fatty acids include $\alpha$-linolenic acid (ALA), eicosapentaenoic acid (EPA) and docosahexaenoic acid (DHA). Omega-6 fatty acids include arachidonic acid and linoleic acid. The differences between these two types of PUFAs are determined by the location of the first double bond in their chemical structures. Omega-3 fatty acids are named as such because the sight of the first double bond in the fatty acid chain occurs at the $3^{\text {rd }}$ carbon. Comparatively, omega- 6 fatty acids first double bond is found at the $6^{\text {th }}$ carbon. This seemingly small difference in structure appears to have dramatic implications on metabolism and integration in the body.

It has been suggested that the risk of developing cardiovascular disease decreases as the ratio of omega- 6 to omega- 3 fatty acids decreases, with the ideal ratio being 1:1. Currently, the typical American diet consumes a 15:1 omega-6/omega-3 ratio (Simopoulos, 2008). It has been hypothesized that a lack of omega-3 fatty acids in the diet may be contributing to the high incidence of cardiovascular disease found in the United States. This has led to a strong push for the general population to incorporate more foods containing omega- 3 fatty acids in them, such as fish, flax seed, certain nuts and vegetable oils (Kris-Etherton et al., 2003). The omega-3 fatty acids EPA and DHA are commonly found in marine animals. These are essential fatty acids, meaning they cannot be synthesized by the human body. The beneficial effects marine oils in reducing cardiovascular disease are believed to be through improved lipoprotein composition, endothelial and smooth muscle function, atherosclerotic plaque build-up, inflammatory mediators and peripheral nervous system function. Three of these, anti-inflammatory response, endothelial proliferation and the peripheral nervous system, are believed to be the primary contributors to lowering arterial blood pressure (Das, 2000; Abeywardena \& Head, 2001). 
Table 1.1. Examples of saturated and unsaturated fatty acids.

Name
Saturated
Palmitic Acid
Unsaturated

Formulas presented as number of carbons in the chain:number of double bonds (carbon at which the first double bond occurs); n-3, omega-3 fatty acid; n-6, omega- 6 fatty acid

EPA and DHA are "less" inflammatory, more so than anti-inflammatory. The structures of omega-3 fatty acids, and products from their breakdown and integration into biological cells are the first processes that lead to their influence on the inflammation process. The source of PUFA's health benefits lies in the multiple double bonds in the chemical structures. A greater number of double bonds lead to an increased fluidity in the cell membranes (De Caterina et al., 1998; Hashimoto et al., 1999). This altered membrane structure triggers the formation of specific types of cytokines and inflammatory mediators such as prostaglandins, tumor necrosis factor and interleukins (Endres et al., 1989; De Caterina et al., 2000). These molecules are inflammatory triggering products, but the properties of the structures of the mediators derived from EPA have anti-inflammatory properties compared to arachidonic acid. For example, arachidonic acid, an omega- 6 fatty acid, is the precursor to active inflammation mediators prostaglandin $P G_{2}$ and thromboxane $T X B_{2}$. The omega-3 fatty acid EPA is the precursor to $\mathrm{PG}_{3}$ and $\mathrm{TXB}_{3}$ which are less active inflammatory agents (Terano et al., 1986). In this way, omega-3 fatty acids are less-inflammatory when compared to their 
omega- 6 counterparts. Additionally, changes in the inflammatory response may have an effect on localized endothelial function.

Peripheral vasculature is highly influenced by endothelial function. Fish oils are suggested to improve endothelial function. Endothelial tissue can contribute to local vasodilation as well as constriction. For example, angiotensin II is a known vasoconstrictor while nitric oxide is a vasodilator. It is believed that an imbalance in these vasoactive actions is one of the causes of endothelial dysfunction and hypertension. Vasoactive agents such as angiotensin II, nitric oxide, endothelial derived hyperpolarizing factor, eicosanoids and endotholin are believed to be influenced by EPA and DHA (Gibbons, 1997; Harris et al., 1997; Noll et al., 1997).

Another potential mechanism, and the primary focus of this thesis, is that fish oil decreases blood pressure through the parasympathetic and sympathetic nervous systems. Although the exact mechanisms for these affects are unclear, it has been suggested that omega- 3 fatty acids have a direct influence on peripheral nervous system function.

\subsection{Summary and Hypotheses}

The purpose of this dissertation is two-fold. First, we aim to compare the neurovascular responses to mental stress in prehypertensive and normotensive humans. Second, the effects of omega-3 fatty acid supplementation will be used to determine if there is a possible health benefit to fish oil supplementation in regards to blood pressure regulation and prevention of cardiovascular disease, specifically hypertension. The link between mental stress and possible mechanisms in the development of hypertension has led us to propose the following hypotheses.

Hypothesis 1: Previously, it has been shown that young borderline hypertensive subjects demonstrated an augmented pressor response during mental stress compared to normotensives (Santangelo et al., 1989; Matsukawa et al., 1991; Jern et al., 1995). Mechanisms suggested to contribute to the augmented pressor response in borderline hypertensives include a blunted forearm vasodilation response (Santangelo et al., 1989) and augmented MSNA responses to mental stress when compared to normotensives (Matsukawa et al., 1991). The neural and vascular responses to mental stress have not been examined collectively in these populations. Additionally, based on recent evidence 
regarding MSNA responses to mental stress (Carter \& Ray, 2009), the results reported by (Matsukawa et al., 1991) may not be representative of the majority of the population. Therefore, in Study 1 we aimed to comprehensively investigate blood pressure, heart rate, MSNA and vascular responses to mental stress. We hypothesized that young prehypertensive males would elicit an augmented blood pressure and MSNA response, but a blunted vasodilation response to mental stress compared to normotensives.

Hypothesis 2: Psychological stress is a known contributor to the development of hypertension (Esler et al., 2008). Though the mechanisms underlying the development of this cardiovascular disease are not fully understood, it has been suggested that omega-3 fatty acid supplementation via fish oil may contribute to the prevention and treatment of hypertension (Appel et al., 1993; Morris et al., 1993; Mori, 2006). Studies investigating omega-3 fatty acids and mental stress have suggested blunted sympathetic activity (i.e., epinephrine) as a mechanism by which blood pressure may be lowered (Delarue et al., 2003). However, direct measurements of sympathetic neural activity have not been investigated during mental stress, and no studies have examined prehypertensive responses to mental stress following omega-3 supplementation. In Study 2 we hypothesized that supplementation with omega- 3 fatty acids, specifically fish oil, would lower resting blood pressure in prehypertensive subjects compared to normotensive. Furthermore, we hypothesized that fish oil would blunt the blood pressure and MSNA, and augment the vascular responses to mental stress in these same prehypertensive individuals compared to normotensives. 


\section{Chapter 2}

\section{Neurovascular Responses to Mental Stress in Prehypertensive Humans}

The material contained in this chapter was previously published in the Journal of Applied Physiology

Schwartz, CE, Durocher, JJ, Carter, JR. (2011). Neurovascular responses to mental stress in prehypertensive humans. Journal of Applied Physiology 10, 76-82. Doi: 10.1152/japplphysiol.00912.2010

\subsection{Introduction}

Mental stress has been linked to several cardiovascular diseases, including hypertension (Esler et al., 2003; Esler et al., 2008). Hypertensive and prehypertensive individuals, as well as normotensives with a family history of hypertension, have all demonstrated an augmented pressor response to mental stress (Falkner et al., 1979; Santangelo et al., 1989; Matsukawa et al., 1991; Widgren et al., 1992; Jern et al., 1995). Although this hypertensive response is well documented, few studies have examined the mechanisms underlying this response.

Mental stress consistently induces forearm vasodilation (Blair et al., 1959; Barcroft et al., 1960; Roddie, 1977; Carter et al., 2005), and evidence suggests that prehypertension blunts this response (Santangelo et al., 1989). The mechanism(s) responsible for this attenuated response remain unresolved, but an augmented sympathetic neural response has been suggested. Specifically, Matsukawa et al. (Matsukawa et al., 1991) reported that MSNA responses to mental stress were augmented in borderline hypertensives when compared to normotensives. Unfortunately, concurrent vascular responses were not assessed (Matsukawa et al., 1991) and MSNA responses to mental stress are highly variable (Carter \& Ray, 2009).

Therefore, the present study aims to determine both forearm vascular and MSNA responses to mental stress in prehypertensive and normotensive adults. We hypothesize that prehypertension will blunt forearm vasodilation and augment MSNA responses to mental stress. Understanding the mechanisms responsible for the augmented pressor response to mental stress is clinically relevant, and may lead to better intervention strategies to help prevent, or at least delay, the development of prehypertension and/or hypertension. 


\subsection{Methods}

\subsubsection{Subjects}

35 healthy men (18 normotensive, age $23 \pm 2$ yrs; 17 prehypertensive, age $22 \pm$ 1 yrs) participated in the study. Normotensive subjects were defined as having a resting systolic pressure less than $120 \mathrm{mmHg}$ and diastolic pressure less than $80 \mathrm{mmHg}$. Prehypertensive subjects were defined as having a resting systolic pressure of 120-139 $\mathrm{mmHg}$ and/or a diastolic pressure of $80-89 \mathrm{mmHg}$. This is consistent with current blood pressure classifications (Chobanian et al., 2003). Subject exclusion criteria included smoking, diabetes, use of blood pressure medication, and autonomic dysfunction. Normotensive $\left(24 \pm 1 \mathrm{~kg} / \mathrm{m}^{2}\right)$ and prehypertensive $\left(26 \pm 1 \mathrm{~kg} / \mathrm{m}^{2}\right)$ subjects had similar body mass indices $(P=0.13)$. Subjects were asked to refrain from exercise, caffeine and alcohol for 12 hours prior to being tested. This experimental protocol was approved by the Michigan Technological University Institutional Review Board (Approval Protocol No. M0172), and all participants signed an informed consent form.

\subsubsection{Experimental Design}

Subjects reported to the laboratory on the three consecutive days. Testing occurred at the same time of day to avoid diurnal fluctuations in autonomic measurements. Resting blood pressures were measured in the seated position three times after 5 minutes of rest on each of the three consecutive days, and reported as the mean. Height and weight were recorded following the resting blood pressure readings on the third day. After resting measurements were taken on day three, subjects were instrumented for the mental stress autonomic function test, which included 5 minutes of supine rest (baseline), 5 minutes of mental stress (mental arithmetic), and 5 minutes of supine rest (recovery). Mental arithmetic consisted of subtracting the number 6 or 7 from a 2-3 digit number continuously as investigators encouraged the subject to respond quickly. The 2-3 digit number was changed every 5-10 seconds. MSNA, heart rate (HR), beat-to-beat blood pressure, forearm and calf blood flow were recorded throughout the protocol. 


\subsubsection{Measurements}

Arterial blood pressure was measured using two techniques. Resting arterial blood pressure was measured three times (separated by approximately one minute intervals) over three consecutive days using an automated sphygmomanometer and reported as a mean value (i.e., 9 readings over 3 days). Beat-to-beat arterial blood pressure was recorded continuously via Finometer (Finapres Medical Systems, Amsterdam, The Netherlands) during the mental stress protocol (i.e., baseline, mental stress, and recovery). The Finometer accurately determines relative changes in arterial blood pressure, but should not be used to determine absolute values. Therefore, the Finometer was used to determine precise changes in arterial blood pressure that occurred during mental stress, while the sphygmomanometer allowed us to compare baseline arterial blood pressures (Table 1). Arterial blood pressures are expressed as systolic (SAP), diastolic (DAP), and mean (MAP) arterial pressures. Three day average MAP is calculated using the classic formula MAP = DAP + 0.333 (SAP - DAP). Supine baseline measurements of MAP are generated by each cardiac cycle waveform produced by the Finometer. HR was recorded with the automated sphygmomanometer during the 3 consecutive days of blood pressure monitoring, and with a three-lead electrocardiogram during the mental stress protocol.

Forearm and calf blood flow were measured using venous occlusion plethysmography. Changes in forearm and calf blood flow were measured via mercuryin-silastic strain gauges placed around the subject's forearm and calf at the point of greatest circumference. Cuffs were placed around the subjects left wrist, upper arm, thigh and ankle. The wrist and ankle cuffs were inflated to $220 \mathrm{mmHg}$ to occlude blood flow to the hand and foot, while the upper arm and thigh cuffs were inflated to $60 \mathrm{mmHg}$ for 8 seconds and deflated for 7 seconds (i.e. 15 second blood flow intervals).

Multifiber recordings of MSNA were made by inserting a tungsten microelectrode into the peroneal nerve of a resting leg. A reference electrode was inserted subcutaneously $2-3 \mathrm{~cm}$ from the recording electrode. Both electrodes were connected to a differential preamplifier, and then to an amplifier (total gain of 80,000) where the nerve signal was band-pass filtered $(700-2000 \mathrm{~Hz})$, and integrated (time constant, 0.1$)$ to obtain a mean voltage display of nerve activity. Satisfactory recordings of MSNA were defined by spontaneous, pulse synchronous bursts that increased during end-expiratory 
apnea, and did not change during auditory stimulation. A loss or shift of the neurogram during mental stress prevented analysis of MSNA data in 14 subjects; thus we report MSNA burst frequency responses to mental stress in a total of 21 subjects (10 normotensive, 11 prehypertensive). Total MSNA responses to mental stress are presented for 19 subjects ( 9 normotensive, 10 prehypertensive).

\subsubsection{Data Analysis}

Data were imported and analyzed in the WinCPRS software program (Absolute Aliens, Turku, Finland). R-waves were detected and marked in the time series. Bursts of MSNA were automatically detected on the basis of amplitude using a signal-to-noise ratio of $3: 1$, within a $0.5 \mathrm{~s}$ search window centered on a $1.3 \mathrm{~s}$ expected burst peak latency from the previous R-wave. Potential bursts were displayed and edited by one investigator. The average burst area occurring during baseline was normalized to a mean value of 100. MSNA was expressed as bursts per minute, bursts per 100 heart beats, and total MSNA (i.e., the sum of the normalized burst areas per minute).

Forearm and calf blood flows were analyzed as percent change, and used to calculate vascular resistance and vascular conductance. Vascular resistance was calculated as MAP divided by limb blood flow, while vascular conductance was calculated as the reciprocal (i.e., limb blood flow divided by MAP).

\subsubsection{Statistical Analysis}

All data were analyzed statistically using commercial software (SPSS 15.0, SPSS Inc., Chicago, Illinois, USA). A two-way repeated measures ANOVA was utilized to determine if changes in MSNA, SAP, DAP, MAP, HR, forearm and calf vascular resistance and conductance occurred during baseline and mental stress, and across trial groups (normotensive and prehypertensive). Post-hoc analyses were performed using least significant difference pairwise comparisons. Resting variables were compared using independent t-tests. Means were considered significantly different when $P<0.05$.

All results are expressed as mean \pm SE. Hemodynamic variables are presented as 5 min averages in results text and 1 min averages in figures to provide more detail on differences between groups. Although there were group differences in forearm vascular responses, data are only presented as $5 \mathrm{~min}$ averages as this was more conducive to 
the sampling technique (venous occlusion plethysmography). Finally, MSNA and calf vascular responses were not different between groups regardless of analysis (i.e., minute by minute vs. 5 min averages), so data are presented as 5 min averages.

\subsection{Results}

Table 2.1 reports seated resting blood pressure and heart rate values taken over three consecutive days. Resting SAP, DAP, and MAP were greater in prehypertensive than normotensive subjects, while resting HR was not different across groups. Table 2.2 reports supine baseline MSNA, along with limb blood flow, vascular resistance, and vascular conductance to the forearm and calf. Forearm blood flow was significantly greater in prehypertensive compared to normotensive subjects, while all other baseline variables were not different between groups (Table 2.2).

Figure 2.1 demonstrates that increases in SAP $(\Delta 9 \pm 2 \mathrm{mmHg}$ vs. $\Delta 14 \pm 2$ $\mathrm{mmHg} ; \mathrm{P}<0.05)$, DAP $(\Delta 8 \pm 1 \mathrm{mmHg}$ vs. $\Delta 11 \pm 1 \mathrm{mmHg} ; \mathrm{P}<0.05)$ and $\operatorname{MAP}(\Delta 10 \pm 1$ $\mathrm{mmHg}$ vs. $\Delta 14 \pm 1 \mathrm{mmHg} ; \mathrm{P}<0.05)$ during mental stress were significantly greater in prehypertensive compared to normotensive subjects (condition $x$ group interaction for $\triangle \mathrm{SAP}$ and $\triangle \mathrm{MAP}$ were significant at $\mathrm{P}<.05$, condition $\mathrm{x}$ group interaction for $\triangle \mathrm{DAP}$ was considered significant at $P=.065)$.

Table 2.1. Resting hemodynamics for normotensive and prehypertensive subjects.

Normotensive

Prehypertensive

\begin{tabular}{lcccccccc} 
Variable & Day 1 & Day 2 & Day 3 & Mean & Day 1 & Day 2 & Day 3 & Mean \\
\hline SAP $(\mathrm{mmHg})$ & $113 \pm 2$ & $112 \pm 1$ & $111 \pm 1$ & $112 \pm 1$ & $127 \pm 2^{*}$ & $126 \pm 2^{*}$ & $127 \pm 2^{*}$ & $127 \pm 2^{*}$ \\
DAP $(\mathrm{mmHg})$ & $65 \pm 2$ & $64 \pm 2$ & $64 \pm 2$ & $64 \pm 1$ & $71 \pm 2^{*}$ & $71 \pm 2^{*}$ & $70 \pm 2^{*}$ & $71 \pm 2^{*}$ \\
MAP (mmHg) & $81 \pm 1$ & $80 \pm 1$ & $80 \pm 1$ & $80 \pm 1$ & $90 \pm 2^{*}$ & $89 \pm 2^{*}$ & $89 \pm 2^{*}$ & $89 \pm 2^{*}$ \\
HR (beats/min) & $69 \pm 3$ & $68 \pm 2$ & $68 \pm 2$ & $68 \pm 2$ & $75 \pm 3$ & $74 \pm 3$ & $76 \pm 3$ & $75 \pm 3$
\end{tabular}

Values are mean \pm SE ( $n=18$ for normotensive, and $n=17$ for prehypertensive). SAP, systolic arterial pressure; DAP, diastolic arterial pressure; MAP, mean arterial blood pressure; HR, heart rate; *Significantly different $(P<0.05)$ from corresponding normotensive value. Measurements were recorded after 5 minutes of seated rest at the same time of day over three consecutive days with an automated sphygmomanometer. 
Table 2.2. Baseline values for normotensive and prehypertensive subjects.

\begin{tabular}{lccl} 
Variable & Normotensive & Prehypertensive & P value \\
\hline MSNA (bursts/min) & $10 \pm 2$ & $11 \pm 2$ & $\mathrm{P}=0.78$ \\
MSNA (bursts/100 hb) & $17 \pm 3$ & $16 \pm 3$ & $\mathrm{P}=0.88$ \\
FBF $(\mathrm{mL} / 100 \mathrm{~mL} / \mathrm{min})$ & $2.5 \pm 0.3$ & $3.4 \pm 0.3^{*}$ & $\mathrm{P}=0.03$ \\
FVR $(\mathrm{mmHg} / \mathrm{mL} / 100 \mathrm{~mL} / \mathrm{min})$ & $35 \pm 2$ & $30 \pm 4$ & $\mathrm{P}=0.28$ \\
$\mathrm{FVC}(\mathrm{mL} / 100 \mathrm{~mL} / \mathrm{min} / \mathrm{mmHg})$ & $0.03 \pm 0.003$ & $0.04 \pm 0.004$ & $\mathrm{P}=0.13$ \\
$\mathrm{CBF}(\mathrm{mL} / 100 \mathrm{~mL} / \mathrm{min})$ & $2.1 \pm 0.2$ & $2.4 \pm 0.2$ & $\mathrm{P}=0.25$ \\
$\mathrm{CVR}(\mathrm{mmHg} / \mathrm{mL} / 100 \mathrm{~mL} / \mathrm{min})$ & $42 \pm 3$ & $42 \pm 4$ & $\mathrm{P}=0.99$ \\
$\mathrm{CVC}(\mathrm{mL} / 100 \mathrm{~mL} / \mathrm{min} / \mathrm{mmHg})$ & $0.03 \pm 0.002$ & $0.03 \pm 0.002$ & $\mathrm{P}=0.80$ \\
\hline Values are $\mathrm{mean} \pm \mathrm{SE}$. MSNA, muscle sympathetic nerve activity ( $\mathrm{n}=15$ for \\
normotensive and $\mathrm{n}=16$ for prehypertensive); FBF, forearm blood flow ( $\mathrm{n}=17$ for \\
normotensive and $\mathrm{n}=16$ for prehypertensive); FVR, forearm vascular resistance; \\
FVC, forearm vascular conductance; CBF, calf blood flow $(\mathrm{n}=17$ for normotensive \\
and $\mathrm{n}=15$ for prehypertensive); CVR, calf vascular resistance; CVC, calf vascular \\
conductance; *Significantly different between groups.
\end{tabular}

Figure 2 demonstrates that mental stress significantly increased MSNA from baseline when expressed as bursts per minute (normotensive: $11 \pm 2$ to $18 \pm 3$ bursts/min, $\mathrm{P}<0.05$; prehypertensive: $11 \pm 3$ to $18 \pm 3$ bursts/min, $P<0.05$ ) or total MSNA (normotensive: $3918 \pm 718$ to $10064 \pm 1295$ a.u., $\mathrm{P}<0.05$; prehypertensive: $5591 \pm 1360$ to $13417 \pm 2684$ a.u., $P<0.05)$. In contrast to blood pressure, MSNA responses to mental stress were not different between groups (Figure 2.2).

Figure 2.3 represents the forearm blood flow responses during mental stress in normotensive and prehypertensive subjects. Although mental stress increased forearm blood flow in both groups, increases were significantly blunted in prehypertensive subjects $(\Delta 116 \pm 16 \%$ vs. $\Delta 62 \pm 11 \%$; $\mathrm{P}<0.01)$. Likewise, decreases in forearm vascular resistance $(\Delta-41 \pm 4 \%$ vs. $\Delta-18 \pm 5 \% ; P<0.01)$ and increases in conductance $(\Delta 95 \pm$ $14 \%$ vs. $\Delta 37 \pm 8 \% ; \mathrm{P}<0.001)$ during mental stress were blunted in prehypertensive subjects. In contrast, calf blood flow $(\Delta 43 \pm 11 \%$ vs. $\Delta 36 \pm 6 \%)$, resistance $(\Delta-12 \pm 5 \%$ vs. $\Delta-8 \pm 4 \%)$ and conductance $(\Delta 29 \pm 10 \%$ vs. $\Delta 19 \pm 5 \%)$ responses during mental stress were similar between groups (Figure 2.4). 

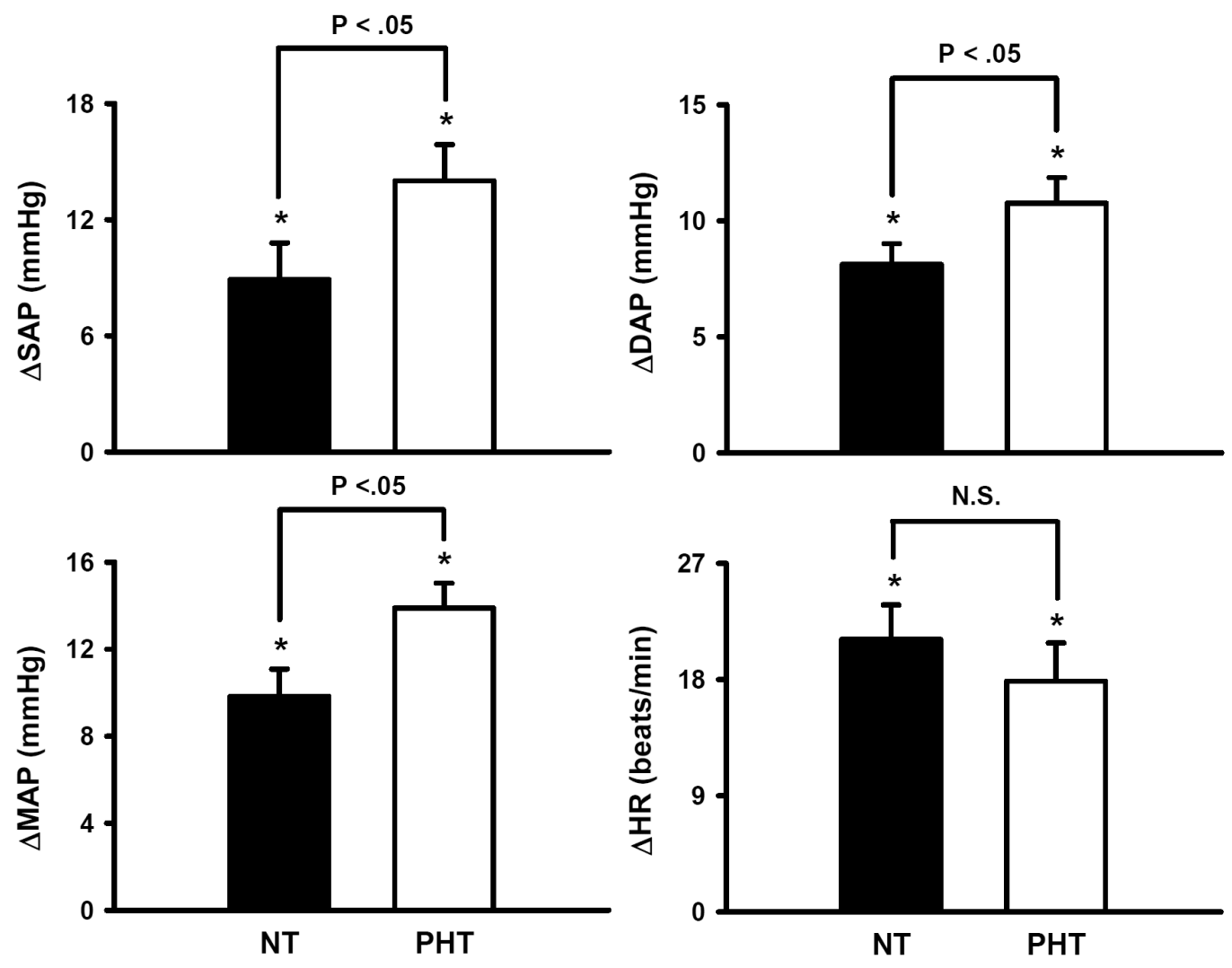

Figure 2.1. Changes in SAP, DAP, MAP and HR during 5 minutes of mental stress in normotensive, NT $(n=18)$ and prehypertensive, PHT $(n=16)$ subjects. Mental stress elicited a significant pressor response in both groups, and this response was augmented in PHT subjects. Mental stress increased HR in both NT and PHT subjects, and there was no significant difference between groups. ${ }^{*} \mathrm{P}<0.05$ from corresponding NT value.
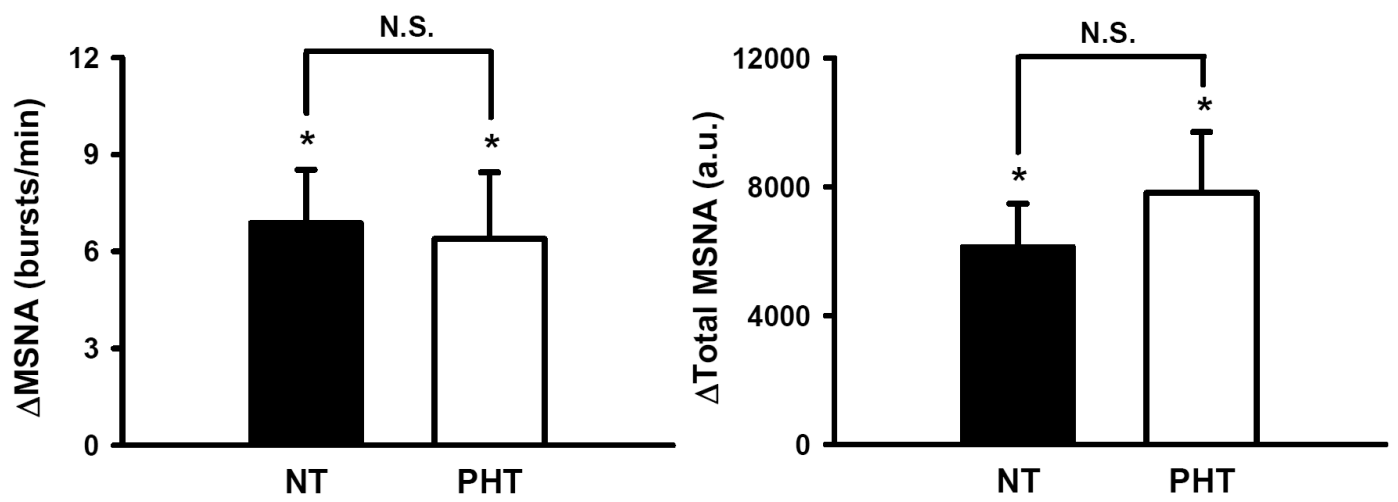

Figure 2.2. Changes in MSNA bursts per minute and total MSNA during 5 minutes of mental stress in normotensive (NT) and prehypertensive (PHT) subjects. Mental stress increased MSNA burst frequency and total MSNA in both groups. These responses were not significantly different between groups (burst frequency: time $\times$ group, $P=0.43$; total MSNA: time $\times$ group, $P=0.29$ ). ${ }^{*} \mathrm{P}<0.05$ from baseline; N.S., no significance; a.u., arbitrary units per minute. 

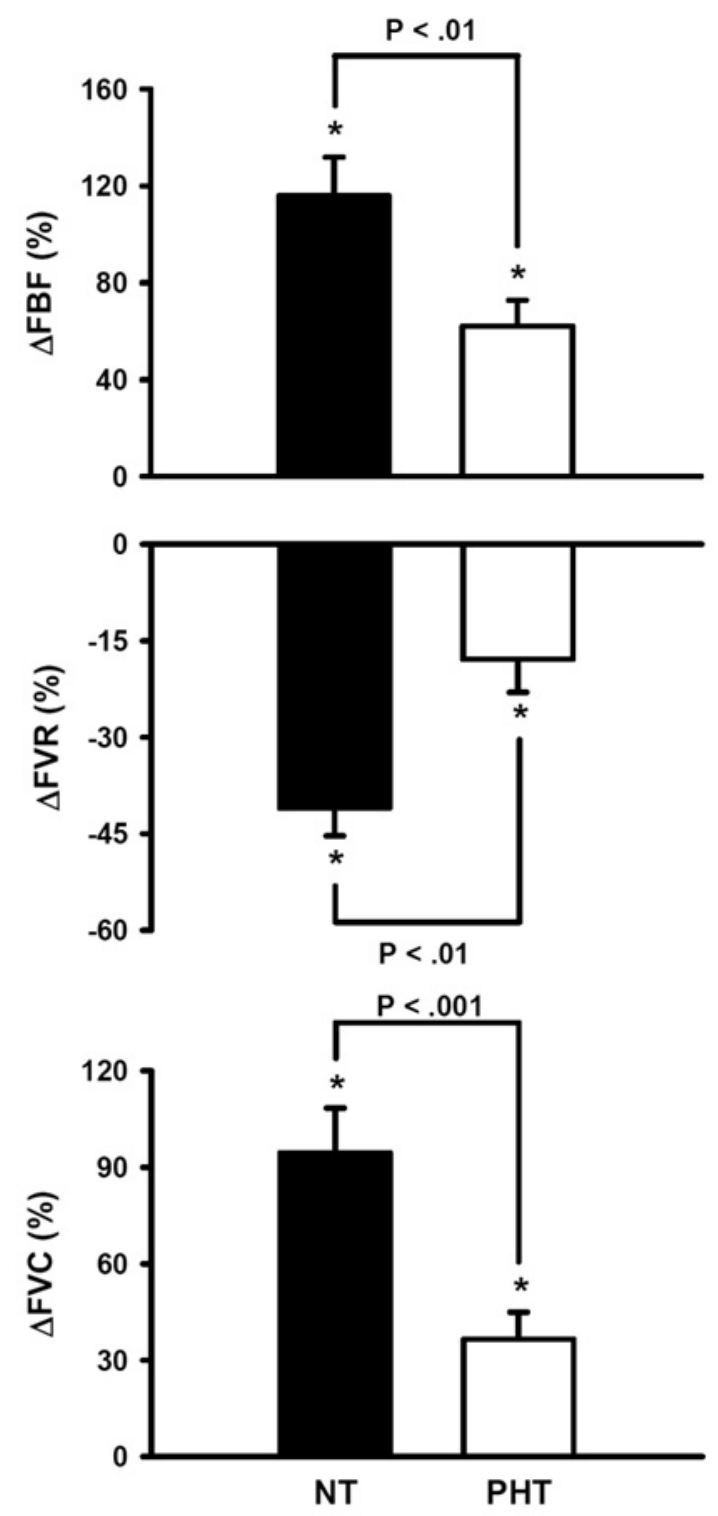

Figure 2.3. Changes in forearm blood flow (FBF), vascular resistance (FVR) and vascular conductance (FVC) during 5 minutes of mental stress. Mental stress elicited forearm vasodilation in both groups, but these responses were blunted in prehypertensive, PHT $(n=15)$ compared to normotensive, NT $(n=17)$ subjects (time $\times$ group $=P<0.01$, all). ${ }^{*} P<0.05$ from baseline. 

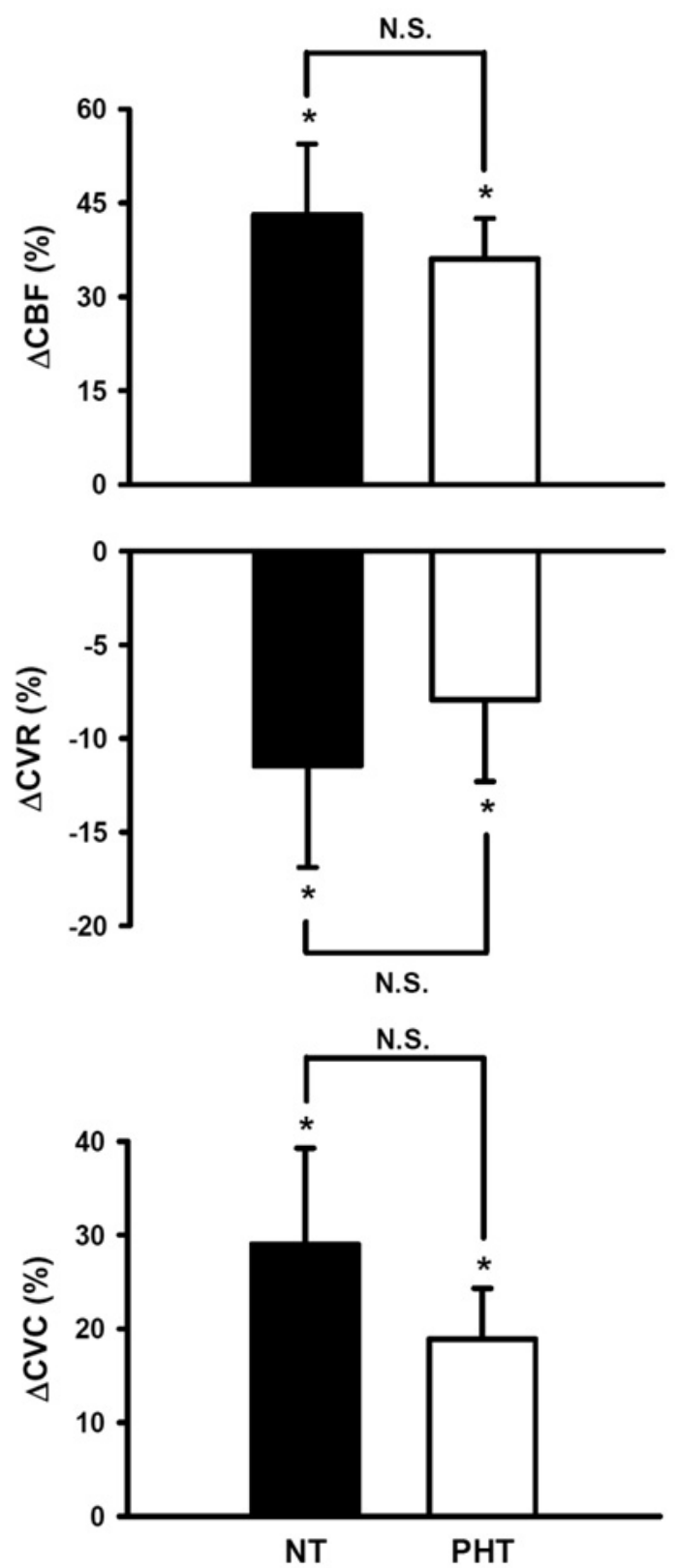

Figure 2.4. Changes in calf blood flow (CBF), vascular resistance (CVR) and vascular conductance (CVC) during 5 minutes of mental stress. Mental stress elicited calf vasodilation in normotensive, NT $(n=17)$ and prehypertensive, PHT $(n=14)$ groups, and these responses were not different between groups (time $\times$ group, $P>0.20$, all). ${ }^{*} P<0.05$ from baseline, N.S., no significance. 


\subsection{Discussion}

The present study compared neurovascular responses to mental stress in prehypertensive and normotensive subjects, and our data reveal three major findings. First, mental stress elicited an augmented pressor response in prehypertensive subjects. Second, this augmented pressor response was associated with an attenuated forearm vasodilation. These findings are consistent with previous work (Santangelo et al., 1989; Matsukawa et al., 1991; Jern et al., 1995). Third, prehypertension did not alter MSNA responses to mental stress. This finding is novel and provides new mechanistic insight into the well documented, yet poorly understood, link between mental stress and hypertension. Collectively, our data indicate that blunted forearm vasodilation, not augmented MSNA, contributes to a more dramatic increase of arterial blood pressure during mental stress in prehypertensive subjects.

Several studies have shown augmented blood pressure responses to mental stress in prehypertensive compared to normotensive subjects (Santangelo et al., 1989; Matsukawa et al., 1991; Jern et al., 1995). Similar results have also been seen in subjects with a family history of hypertension (Widgren et al., 1992; Noll et al., 1996). Furthermore, an augmented pressor response to stress in normotensives may even help predict the development of hypertension (Matthews et al., 2004; Esler et al., 2008). In the present study, we report an augmented pressor response to mental stress in prehypertensive subjects. Thus, our findings are consistent with previous studies and strengthen the rationale to determine potential mechanisms that may be responsible for this augmented response.

Matsukawa et al. (Matsukawa et al., 1991) reported an elevated blood pressure response to mental stress in borderline hypertensives, and suggested that the difference was likely due to a decrease in MSNA in normotensives and no change in the borderline hypertensives. However, recent evidence (Carter \& Ray, 2009) suggests that the findings of Matsukawa et al. (Matsukawa et al., 1991) may not represent typical MSNA responses to mental stress. Specifically, a recent retrospective analysis of 82 neurograms from normotensive subjects indicated that nearly $90 \%$ of subjects demonstrated an increase or no change in MSNA, while $\sim 10 \%$ were classified as "negative" MSNA responders ( $\leq \Delta-3$ bursts/min) (Carter \& Ray, 2009). Therefore, the findings of Matsukawa et al. (Matsukawa et al., 1991), which reported a significant 
sympathoinhibition of MSNA during mental stress in normotensive subjects, may not represent a normal distribution with regards to typical MSNA responses to mental stress. The present study demonstrates similar increases of MSNA during mental stress in normotensive and prehypertensive patients. We attribute differences between our data and the findings of Matsukawa et al. (Matsukawa et al., 1991) to the inherent variability of MSNA responses to mental stress in humans (Carter \& Ray, 2009). However, it is important to note that in the present study, 10 of 10 normotensive subjects and 10 of 11 prehypertensive subjects demonstrated an increase or no change in MSNA during mental stress. These ratios are consistent with a recent large-scale study that reported MSNA either increases or does not change during mental stress in approximately $90 \%$ of adults (Carter \& Ray, 2009). Therefore, we are confident in our data and conclude that MSNA responses to mental stress are similar in normotensive and prehypertensive populations.

Whereas prehypertension did not alter MSNA responses to mental stress, it did alter forearm vascular responses. Specifically, prehypertension blunted the classic forearm vasodilatory response associated with mental stress. Blunted forearm vascular responses to physiological stressors are associated with increased incidence or risk of hypertension (Takeshita et al., 1982; Santangelo et al., 1989; Boutcher et al., 2009). For example, young normotensive individuals with a family history of hypertension display a reduction in peak forearm blood flow (Boutcher et al., 2009) and an increased forearm vascular resistance during (Takeshita et al., 1982) and after (Boutcher et al., 2009) reactive hyperemia. Moreover, borderline hypertensives demonstrate increased forearm vascular resistance and MAP during mental stress (Santangelo et al., 1989). Thus the present study, which demonstrates blunted forearm vascular responses to mental stress in prehypertensive subjects, is consistent with previous work (Santangelo et al., 1989) and offers new insights by demonstrating that the blunted forearm vascular response to mental stress is not accompanied by altered MSNA.

A recent study reports similar MSNA responses to mental stress in the arm and leg (Carter et al., 2005), making it reasonable to assume that other mechanisms beyond MSNA may be responsible for the blunted forearm vasodilation to mental stress in our prehypertensive subjects. However, it should be noted that other studies have reported a divergence in arm and leg MSNA (Anderson et al., 1987) and decreases of arm MSNA 
(Halliwill et al., 1997) during mental stress. We recognize the absence of arm MSNA data as a limitation to the present study, but do not believe this lessens the impact of the data. Our primary focus remains on mechanisms responsible for the augmented pressor response to mental stress in prehypertensive subjects, not the mechanisms underlying forearm vasodilation. Both leg MSNA and forearm vascular conductance have been independently proposed as potential mechanisms contributing to the augmented pressor response, and the present study advanced our knowledge by demonstrating that blunted forearm vasodilation, not augmented MSNA, is a primary contributor. This is important as it had previously been assumed that augmented MSNA was a likely contributor (Matsukawa et al., 1991). However, we recognize that other potential mechanisms might include vasoconstriction to non-muscular beds, including renal, splanchnic and skin (Brod, 1963; Wallin et al., 1973; Tidgren \& Hjemdahl, 1989; Kuipers et al., 2008); future work will have to address these vascular beds.

As previously noted, passive MSNA withdrawal has been suggested as a potential mechanism for the forearm vasodilation associated with mental stress (Halliwill et al., 1997), but this remains debatable (Carter et al., 2005) and other mechanisms have been proposed (Dietz et al., 1994; Lindqvist et al., 1996; Cardillo et al., 1997). Specifically, both nitric oxide (Dietz et al., 1994; Cardillo et al., 1997; Cardillo et al., 1998) and circulating epinephrine (Lindqvist et al., 1996) have been shown to contribute to forearm vasodilation during mental stress. Of interest to the present study, Cardillo et al. (Cardillo et al., 1998) reported that nitric oxide mediated vasodilation during mental stress was attenuated in hypertensives, and this could be due to possible endothelial dysfunction. We did not assess nitric oxide levels in the current study, but it seems reasonable to speculate that the blunted forearm vasodilation during mental stress in prehypertensive subjects may be related to altered nitric oxide responses.

Although mental stress does not typically modulate calf vasculature (Rusch et al., 1981; Carter et al., 2005), calf vasodilation has been observed during mental stress (Kuipers et al., 2008). Therefore, the modest, yet significant, calf vasodilation reported in the present study is reasonable and consistent with prior work. Importantly, calf vasodilatory responses to mental stress were similar in normotensive and prehypertensive subjects. Thus, prehypertension appears to elicit divergent limb vascular responses to mental stress. The clinical consequence of this divergent vascular 
response remains unclear, but it is consistent with other studies reporting divergent limb vascular responses during physiological stessors in both animals (Wilson et al., 2006) and humans (Monahan \& Ray, 2002; Lawrence et al., 2010).

In conclusion, the present study demonstrates that prehypertension elicits a more dramatic pressor response to mental stress when compared to normotensive subjects. This augmented pressor response appears to be related to blunted forearm vasodilation, but not augmented MSNA. These findings provide new insight into the complex relationship between mental stress and hypertension.

\subsection{Perspectives}

Despite attempts to recruit young, prehypertensive women, we were only successful in the recruitment of 17 prehypertensive men. Accordingly, we caution the extrapolation of the present data to women. A growing body of evidence suggests that the relations between MSNA and arterial blood pressure regulation differ in men and women (Joyner et al., 2010), and we cannot speculate how these differences might translate to the present findings. Although young, otherwise healthy women have a lower incidence of prehypertension than their young, male counterparts (Izzo, 2007), they have a much higher incidence of anxiety and panic disorder (Westenberg \& Liebowitz, 2004). The long term impact of these more 'chronic' stressors on arterial blood pressure remains unclear, but evidence suggests that such chronic stressors are linked to essential hypertension via autonomic mechanisms (Esler et al., 2008). Whereas the present study focused on neurovascular responses to acute mental stress in prehypertensive men, future investigations might focus on not only prehypertensive women, but also chronic stress.

\subsection{Acknowledgements}

The authors thank Jenna Klein, Ashley Yenior, Kristen Reed, Huan Yang and Sarah Stream for their assistance in data collection and analysis for this project. We also thank all the subjects for their participation and cooperation. This project was supported by National Institutes of Health grants HL-088689 and HL-098676. 


\section{Chapter 3}

\section{Omega-3 Fatty Acids and the Neurovascular Responses to Mental Stress in Humans}

\subsection{Introduction}

Omega-3 fatty acids, particularly eicosapentaenoic acid (EPA) and docosahexaenoic acid (DHA) found in fish oil, have been suggested as a possible nonpharmaceutical treatment of cardiovascular disease (CVD), including hypertension (Burr, 1989; Morris et al., 1993; Marchioli et al., 2002; Thies et al., 2003; Yamagishi et al., 2008). Currently, over 76 million people suffer from high blood pressure (Roger et al., 2011). Hypertension has been shown to increase the risk of stroke, ischemic heart disease and cardiac related mortality (Kannel, 1975; MacMahon et al., 1990; van den Hoogen et al., 2000). A small reduction in blood pressure may have clinical significance on the impact on cardiovascular health (Collins et al., 1990).

Fish oil has been reported to lower blood pressure in hypertensive individuals (Appel et al., 1993; Morris et al., 1993; Geleijnse et al., 2002). However, omega-3 fatty acids demonstrate mixed effects in mild hypertension (Levinson et al., 1990; Singer, 1990; Morris et al., 1993), and do not appear to have a hypotensive influence on individuals with normal blood pressures (Appel et al., 1993; Morris et al., 1993). Collectively, it appears that fish oil may have an increasingly preventative effect as resting blood pressure increases.

Chronic mental stress is a known contributor to hypertension (Esler et al., 2008), and typically elicits augmented neural and cardiovascular responses (Cannon, 1927; Blair et al., 1959; Barcroft et al., 1960; Carter \& Ray, 2009). Augmented sympathetic nerve activity (Esler et al., 1986; Egan et al., 1987; Anderson et al., 1989) and altered peripheral vascular function are two proposed mechanisms contributing to the development of hypertension. Animal studies demonstrate reduced blood pressure, cardiac sympathetic activity and sympathoadrenal responses, as well as improved vascular compliance, following omega-3 supplementation (Bayorh et al., 1989; ChinDusting et al., 1998; Nishimura et al., 2000; Rousseau-Ralliard et al., 2009). However, investigations in humans are less clear. Fish oil has demonstrated both decreases and no change in resting blood pressure (Appel et al., 1993; Morris et al., 1993) and 
sympathetic nerve activity (Delarue et al., 2003; Monahan et al., 2004). Additionally, vascular responses in humans following omega-3 supplementation appear to be improved (Kenny et al., 1992; Mori et al., 2000; Khan et al., 2003). However, few studies have investigated the effect of fish oil in humans during a psychological challenge.

Delarue et al. (2003) reported that circulating catecholamines, an indirect measure of sympathetic neural activity, were reduced during mental stress after fish oil supplementation. More recently, Monahan et al. (2004) measured muscle sympathetic nerve activity (MSNA) responses following omega-3 supplementation and demonstrated augmented responses to physiological stressors, although mental stress was not investigated. Borderline hypertensive subjects have demonstrated augmented MSNA as well as augmented norepinephrine responses to mental stress (Eliasson, 1985; Anderson et al., 1989; Matsukawa et al., 1991). Therefore, investigating the effects of fish oil on MSNA during mental stress may provide information as to the beneficial effects of omega- 3 fatty acids on the development of hypertension.

Recently, the National Heart, Lung and Blood Institute and the American Heart Association introduced prehypertension as a new category of blood pressure classification. These individuals demonstrate a resting blood pressure in between normal and high categories, and represent a population at higher risk of developing hypertension (Vasan et al., 2001; Moreira et al., 2008). Early intervention, such as fish oil supplementation, may play an important role in preventing the onset of hypertension (Fuchs, 2010). However, the mechanisms by which fish oils act in reducing blood pressure in prehypertensive subjects remain unclear.

Therefore, the aim of this study was to investigate MSNA and vascular responses to mental stress before and after omega-3 supplementation in prehypertensive and normotensive individuals. We hypothesized that fish oil would 1) lower resting blood pressure and MSNA in prehypertensive individuals, 2) blunt the blood pressure and MSNA responses to mental stress in the prehypertensive group, and 3) augment vasodilatory responses to mental stress in prehypertensive individuals compared to normotensive. Identifying the mechanism(s) by which omega-3 fatty acids lower blood pressure may help us further understand the development of hypertension, and provide a possible preventative and/or therapeutic measure against the onset of cardiovascular disease. 


\subsection{Methods}

\subsubsection{Subjects}

Thirty-eight normotensive subjects (18 men, 20 women) age $24 \pm 1$ yrs and 29 prehypertensive subjects (28 men, 1 woman) age $24 \pm 1$ yrs participated in this study. Normotension was classified as a resting systolic blood pressure less than $120 \mathrm{mmHg}$ and a resting diastolic blood pressure of $80 \mathrm{mmHg}$. Prehypertension was classified as a resting systolic blood pressure of $120-139 \mathrm{mmHg}$ and/or a diastolic blood pressure of 80-89 mmHg (Chobanian et al., 2003). All subjects signed an informed consent and refrained from caffeine, alcohol and exercise for at least 12 hours before testing. Exclusion criteria for participants included smoking, diabetes, autonomic dysfunction, and use of blood pressure medication. This study was approved by the Michigan Technological University Institutional Review Board (Approval Protocol No. M0172).

\subsubsection{Experimental Protocol}

All subjects were randomly assigned into this double-blind, placebo controlled investigation, and were tested before (pre) and after (post) 8 weeks of fish oil or placebo (olive oil) supplementation. Subjects reported to the Integrative Physiology Laboratory at Michigan Technological University at the same time of day for three consecutive days during the pre- and post-treatment sessions. The protocol for both pre- and posttreatments are outlined in Figure 3.1.

Upon completion of the pre-treatment testing, subjects were randomly assigned into the fish oil or placebo (olive oil) group. Participants ingested 9 grams/day of fish oil pills (1.6g EPA, 1.1g DHA) or 9 grams/day of placebo pills for 8 weeks. A pill diary and regular email and phone reminders were issued to the subjects to track compliance according to the study terms. Subjects were asked to maintain current diet and exercise habits during the 8 week supplementation period. After 8 weeks of treatment, subjects returned to the laboratory for post-treatment testing, and completed the same protocol outlined above (Fig.3.1). Two subjects in the prehypertensive group were unable to complete the post autonomic testing procedure, but did complete the seated resting blood pressure measurements. Therefore, autonomic testing during mental stress is reported for 27 prehypertensive subjects. 


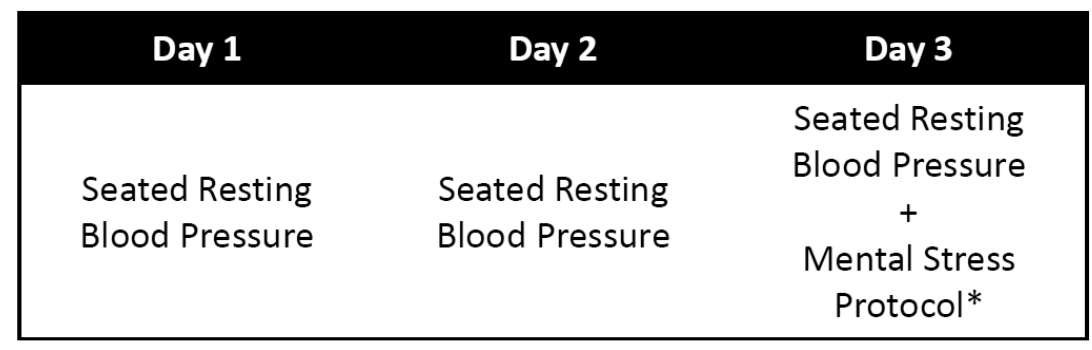

\section{* Mental Stress Protocol}

\begin{tabular}{c|c|c} 
Baseline & Mental Arithmetic & Recovery \\
\hline 5 minutes & 5 minutes & 5 minutes
\end{tabular}

Figure 3.1. The experimental protocol for pre and post testing for subjects. Testing protocols were identical pre and post fish oil or placebo supplementation and were separated by 8 weeks.

\subsubsection{Measurements}

Heart rate was measured using a 3-lead electrocardiogram. Arterial blood pressure was measured utilizing two different methods. Seated resting blood pressures were obtained using the IntelliSense ${ }^{\circledR}$ automated sphygmomanometer (Model HEM907XL, Omron Healthcare, Inc., Bannockburn, IL). A cuff was placed around the upper arm in line with the brachial artery and inflated to approximately $200 \mathrm{mmHg}$ to occlude blood flow to the arm. Pressure was then slowly released allowing for systolic and diastolic arterial pressures to be recorded by the automated sphygmomanometer. Seated resting recordings were taken 3 times each day, separated by 1 minute between readings and following 5 minutes of seated rest. Importantly, all pre- and post-treatment blood pressure measurements were recorded at the same time of day to limit diurnal blood pressure variations as a potential confounder (Richards et al., 1986). Beat to beat arterial pressure was recorded using the Finometer (Finapres Medical Systems, Amsterdam, The Netherlands) on the middle finger throughout autonomic testing. The Finometer is an ideal technique for measuring beat-to-beat changes in arterial pressure, but is not accurate and reproducible in measuring absolute values. Therefore, we used the seated resting brachial arterial pressure as absolute resting values and the Finometer for recording relative changes during mental stress and recovery. 
Multifiber recordings of MSNA were recorded using the microneurography technique. Briefly, a tungsten microelectrode was inserted into the common peroneal nerve located in the popliteal region of the knee or at the base of the fibular head of the lower leg. A reference electrode was inserted subcutaneously 2-3 centimeters away from the recording electrode. Both electrodes were connected to a preamplifier and amplifier. The signal was amplified 80,000 times, band-pass filtered $(700-2000 \mathrm{~Hz})$ and integrated (time constant, $0.1 \mathrm{~s}$ ) to obtain a mean voltage display of nerve activity. MSNA was determined by observing spontaneous multifiber bursts of activity, and confirmed by having the subject perform end-expiratory apnea with a resultant increase in MSNA. The signal was distinguished from skin sympathetic nerve activity by performing auditory stimulation with no subsequent neural reaction. A shift in the neurogram during mental stress prevented recordings in 18 normotensive (11 fish oil, 7 placebo) and 17 prehypertensive ( 9 fish oil, 8 placebo) individuals. Therefore, nerve recordings are reported in only 30 (20 normotensive, 10 prehypertensive) subjects for the mental stress portion of the study.

Arm and leg blood flow were measured using venous occlusion plethysmography (D.E. Hokanson, Inc., Bellevue, WA). Occlusion cuffs were placed around the left upper arm, wrist, thigh, and ankle. The wrist and ankle cuffs were inflated to $220 \mathrm{mmHg}$ in order to temporarily prevent circulation to the hand and foot. The arm and thigh cuffs were then inflated to $60 \mathrm{mmHg}$ for 7 seconds and deflated for 8 seconds (i.e. 4 readings per minute). Inflation of the collecting cuffs allows arterial blood flow into the forearm and calf, but prevents venous flow return. Strain gauges were placed around the greatest circumference of the forearm and calf allowing for direct measurements of changes in arm and leg circumference. Technical issues and artifact during mental stress prevented the analysis of forearm and calf blood flow in several subjects. We were able to record forearm measurements in 29 fish oil subjects and 26 placebo subjects, while in the calf we recorded measurements in 28 fish oil and 25 placebo subjects.

\subsubsection{Data Analysis}

Data were recorded using WinDaq/Pro data acquisition software (DATAQ Instruments Inc., Akron, Ohio), and imported into WinCPRS (Absolute Aliens, Turku, Finland) software package for analysis. R-waves from the electrocardiogram were 
detected and marked in the time series. Integrated bursts of MSNA were detected as a 3:1 burst-noise ratio within a search window of 0.5 seconds based on an average expected burst peak latency of 1.3 seconds following the previous R-wave. Spontaneous bursts of MSNA were normalized to the average burst size during baseline, and designated a value of 100 arbitrary units. Sympathetic bursts of activity were expressed as burst frequency (bursts/minute), burst incidence (bursts/100 heartbeats) and total activity (arbitrary units). Total MSNA was determined as the burst activity multiplied by the average normalized area under the burst. This analysis accounts for the change in the size of the burst per minute. Blood flow data were analyzed using the NIVP3 software (D.E. Hokanson, Inc., Bellevue, WA). Percent changes in flow per time were analyzed in both the forearm and calf. Absolute limb blood flow, as well as limb vascular resistance and conductance were reported. Vascular resistance was calculated as the mean arterial pressure divided by the absolute blood flow measurement, whereas vascular conductance was calculated as the reciprocal of resistance (i.e. blood flow divided by mean arterial pressure).

\subsubsection{Statistical Analysis}

Data are presented as mean \pm SE and analyzed using commercial software SPSS 18.0 (SPSS Chicago, IL). Effects of fish oil and placebo at rest and mental stress were analyzed in the normotensive and prehypertensive groups, respectively. Resting values were compared using a 1-between (fish oil vs. placebo) by 1-within (pre vs. post) repeated measures ANOVA. Statistically significant differences were analyzed post-hoc using paired t-tests. Significance was determined as $P<0.05$.

The mental stress condition was analyzed using a 1-between (fish oil vs. placebo) by 2-within (pre vs. post; baseline vs. mental stress) repeated measures ANOVA. Those analyses that showed a significant $(P<0.05)$ interaction were further analyzed post-hoc using a paired t-test when comparing pre vs. post within groups as well as independent t-tests when analyzing between groups. Prehypertensive and normotensive groups were analyzed separately in order to better compare individual group responses to fish oil or placebo supplementation. These groups were further combined into one group, and analyzed using a 1-between (fish oil vs. placebo) by 2within (pre vs. post; baseline vs. mental stress) repeated measures ANOVA. Any 
interactions that were significantly different were further analyzed post-hoc using a paired t-test.

Mauchly's test of sphericity was performed for all ANOVA analyses in order to test for differences between variances. A significant $(P<0.05)$ result led to the use the Huynh-Feldt (sphericity values greater than 0.75 ) or Greenhouse-Geisser (sphericity values less than 0.75 ) correction factors.

\subsection{Results}

\subsubsection{Resting Baseline Measurements for Prehypertensive and Normotensive Subjects}

Resting blood pressure and heart rate values for normotensive and prehypertensive groups are reported in Tables 3.1 and 3.2 respectively. There were no differences in SAP, DAP, MAP or HR following fish oil or placebo supplementation. Resting forearm and calf vascular measurements are presented in Tables 3.3 and 3.4 for normotensive and prehypertensive groups, respectively. FBF, FVR, FVC, CVR and CVC all showed a significant time effect (pre vs. post) in the normotensive group, but these responses were not different between fish oil and placebo groups. All limb blood flow measurements in the prehypertensive group were not statistically altered by either fish oil or placebo.

Resting MSNA measurements are represented in Fig. 3.2. In the normotensive group, MSNA burst frequencies were not significantly different following either fish oil or placebo supplementation (fish oil, $11 \pm 2$ to $10 \pm 1$ bursts/min; placebo, $10 \pm 2$ to $13 \pm 2$ bursts/min; time, $\mathrm{P}=.73$; time $\mathrm{x}$ drug interaction, $\mathrm{P}=0.16$ ). Similarly, MSNA incidences were not different following either fish oil or placebo supplementation (fish oil, $18 \pm 3$ to $16 \pm 2$ bursts/100 heart beats; placebo, $17 \pm 2$ to $19 \pm 3$ bursts/100 heart beats; time, $\mathrm{P}=0.86$; time $\mathrm{x}$ drug interaction, $\mathrm{P}=0.34$ ). Similar findings were observed in the prehypertensive individuals (Fig. 3.2). 
Table 3.1. Resting hemodynamics before and after 8 weeks of fish oil supplementation or placebo in normotensive adults.

\begin{tabular}{|c|c|c|c|c|c|c|c|c|}
\hline \multirow[b]{2}{*}{ Variable } & \multicolumn{4}{|c|}{ Pre-Treatment } & \multicolumn{4}{|c|}{ Post -Treatment } \\
\hline & Day 1 & Day 2 & Day 3 & Ave & Day 1 & Day 2 & Day 3 & Ave \\
\hline \multicolumn{9}{|c|}{ Normotensive, Fish Oil Group ( $n=19)$ : } \\
\hline $\mathrm{SAP}(\mathrm{mmHg})$ & $110 \pm 2$ & $109 \pm 2$ & $110 \pm 2$ & $110 \pm 1$ & $109 \pm 1$ & $107 \pm 2$ & $106 \pm 3$ & $107 \pm 2$ \\
\hline $\mathrm{DAP}(\mathrm{mmHg})$ & $65 \pm 1$ & $67 \pm 1$ & $66 \pm 1$ & $66 \pm 1$ & $67 \pm 1$ & $67 \pm 1$ & $66 \pm 1$ & $66 \pm 1$ \\
\hline MAP (mmHg) & $80 \pm 1$ & $81 \pm 1$ & $80 \pm 1$ & $80 \pm 1$ & $81 \pm 1$ & $80 \pm 1$ & $75 \pm 4$ & $80 \pm 1$ \\
\hline HR (beats/min) & $71 \pm 2$ & $70 \pm 2$ & $72 \pm 3$ & $71 \pm 2$ & $69 \pm 3$ & $69 \pm 3$ & $75 \pm 3$ & $71 \pm 2$ \\
\hline \multicolumn{9}{|c|}{ Normotensive, Placebo Group ( $n=19)$ : } \\
\hline $\mathrm{SAP}(\mathrm{mmHg})$ & $108 \pm 2$ & $107 \pm 2$ & $105 \pm 2$ & $107 \pm 2$ & $107 \pm 2$ & $106 \pm 2$ & $108 \pm 2$ & $107 \pm 2$ \\
\hline $\mathrm{DAP}(\mathrm{mmHg})$ & $66 \pm 2$ & $65 \pm 2$ & $65 \pm 1$ & $65 \pm 1$ & $68 \pm 1$ & $64 \pm 1$ & $67 \pm 1$ & $66 \pm 1$ \\
\hline MAP $(\mathrm{mmHg})$ & $80 \pm 1$ & $79 \pm 1$ & $78 \pm 1$ & $79 \pm 1$ & $81 \pm 1$ & $78 \pm 1$ & $80 \pm 2$ & $80 \pm 1$ \\
\hline HR (beats/min) & $74 \pm 3$ & $73 \pm 2$ & $75 \pm 2$ & $74 \pm 2$ & $76 \pm 3$ & $73 \pm 3$ & $78 \pm 2$ & $76 \pm 2$ \\
\hline
\end{tabular}

Table 3.2. Resting hemodynamics before and after 8 weeks of fish oil supplementation or placebo in prehypertensive adults.

\begin{tabular}{|c|c|c|c|c|c|c|c|c|}
\hline \multirow[b]{2}{*}{ Variable } & \multicolumn{4}{|c|}{ Pre-Treatment } & \multicolumn{4}{|c|}{ Post -Treatment } \\
\hline & Day 1 & Day 2 & Day 3 & Ave & Day 1 & Day 2 & Day 3 & Ave \\
\hline \multicolumn{9}{|c|}{ Prehypertensive, Fish Oil Group $(n=15)$ : } \\
\hline $\mathrm{SAP}(\mathrm{mmHg})$ & $127 \pm 2$ & $126 \pm 2$ & $126 \pm 1$ & $127 \pm 1$ & $126 \pm 2$ & $124 \pm 1$ & $126 \pm 2$ & $125 \pm 2$ \\
\hline $\mathrm{DAP}(\mathrm{mmHg})$ & $68 \pm 3$ & $69 \pm 3$ & $67 \pm 3$ & $68 \pm 2$ & $70 \pm 2$ & $68 \pm 2$ & $65 \pm 2$ & $68 \pm 2$ \\
\hline MAP $(\mathrm{mmHg})$ & $88 \pm 2$ & $88 \pm 2$ & $87 \pm 2$ & $88 \pm 2$ & $88 \pm 2$ & $87 \pm 1$ & $85 \pm 1$ & $87 \pm 1$ \\
\hline HR (beats/min) & $77 \pm 4$ & $73 \pm 3$ & $76 \pm 4$ & $73 \pm 2$ & $71 \pm 3$ & $74 \pm 3$ & $72 \pm 2$ & $73 \pm 2$ \\
\hline \multicolumn{9}{|c|}{ Prehypertensive, Placebo Group $(n=14)$ : } \\
\hline SAP (mmHg) & $128 \pm 3$ & $126 \pm 2$ & $127 \pm 2$ & $126 \pm 2$ & $123 \pm 2$ & $122 \pm 2$ & $125 \pm 2$ & $123 \pm 2$ \\
\hline $\mathrm{DAP}(\mathrm{mmHg})$ & $73 \pm 2$ & $74 \pm 2$ & $74 \pm 2$ & $74 \pm 2$ & $73 \pm 2$ & $73 \pm 2$ & $74 \pm 2$ & $74 \pm 2$ \\
\hline MAP $(\mathrm{mmHg})$ & $91 \pm 2$ & $91 \pm 2$ & $92 \pm 2$ & $92 \pm 1$ & $90 \pm 2$ & $89 \pm 2$ & $91 \pm 2$ & $90 \pm 2$ \\
\hline HR (beats/min) & $74 \pm 3$ & $71 \pm 3$ & $73 \pm 3$ & $76 \pm 3$ & $72 \pm 4$ & $74 \pm 3$ & $76 \pm 4$ & $74 \pm 3$ \\
\hline
\end{tabular}

Values are mean \pm SE. SAP, systolic arterial pressure; DAP, diastolic arterial pressure; MAP, mean arterial blood pressure; HR, heart rate. All time and time $\times$ drug interactions were $\mathrm{P}>0.05$. 
Table 3.3. Baseline values for normotensive subjects pre and post fish oil or placebo supplementation.

\begin{tabular}{lcc|cc|cc} 
Variable & \multicolumn{2}{c|}{ Fish Oil } & \multicolumn{2}{c|}{ Placebo } & Time & $\begin{array}{c}\text { Time } \mathbf{x} \\
\text { Drug }\end{array}$ \\
& PRE & POST & PRE & POST & Interaction & Interaction \\
\hline FBF (units) & $2.5 \pm 0$ & $2.8 \pm 0$ & $2.2 \pm 1$ & $2.5 \pm 1$ & 0.05 & 0.81 \\
FVR (mmHg/units) & $45 \pm 5$ & $40 \pm 5$ & $49 \pm 5$ & $41 \pm 5^{*}$ & 0.02 & 0.68 \\
FVC & $2.7 \pm 0$ & $3.2 \pm 0^{*}$ & $2.4 \pm 0$ & $2.9 \pm 0^{*}$ & 0.01 & 0.95 \\
$\left(100^{*}\right.$ units/mmHg) & $2.0 \pm 0$ & $2.2 \pm 0$ & $2.2 \pm 0$ & $2.3 \pm 0$ & 0.14 & 0.70 \\
CBF (units) & $54 \pm 4$ & $45 \pm 4^{*}$ & $46 \pm 5$ & $40 \pm 3^{*}$ & 0.01 & 0.56 \\
CVR (mmHg/units) & & & & & 0.01 & 0.93 \\
CVC & $2.1 \pm 0$ & $2.4 \pm 0^{*}$ & $2.5 \pm 0$ & $2.8 \pm 0^{*}$ & 0.01 & \\
\hline (100*units'mmHg) & & & & &
\end{tabular}

Values are mean \pm SE. MSNA, muscle sympathetic nerve activity $(n=16$ for fish oil and $n=17$ for placebo); FBF, forearm blood flow ( $n=18$ for fish oil and $n=18$ for placebo); FVR, forearm vascular resistance ( $n=18$ for fish oil and $n=18$ for placebo); FVC, forearm vascular conductance ( $n=18$ for fish oil and $n=18$ for placebo); CBF, calf blood flow ( $n=16$ for fish oil and $n=16$ for placebo); CVR, calf vascular resistance $(n=16$ for fish oil and $n=16$ for placebo); CVC, calf vascular conductance ( $n=16$ for fish oil and $n=16$ for placebo) *Significantly different from pre value for fish oil or placebo, respectively.

Table 3.4. Baseline values for prehypertensive subjects pre and post fish oil or placebo supplementation.

\begin{tabular}{lcc|cc|cc} 
Variable & \multicolumn{2}{c|}{ Fish Oil } & \multicolumn{2}{c|}{ Placebo } & Time & $\begin{array}{c}\text { Time } \mathbf{x} \\
\text { Drug }\end{array}$ \\
& PRE & POST & PRE & POST & Interaction & Interaction \\
\hline FBF (units) & $3.9 \pm 0$ & $3.5 \pm 0$ & $3.2 \pm 0$ & $3.4 \pm 1$ & 0.80 & 0.37 \\
FVR (mmHg/units) & $27 \pm 3$ & $30 \pm 3$ & $33 \pm 3$ & $37 \pm 6$ & 0.21 & 0.75 \\
FVC & $4.1 \pm 0$ & $3.9 \pm 0$ & $3.4 \pm 0$ & $3.7 \pm 1$ & 0.96 & 0.55 \\
$\begin{array}{l}\text { (100*units/mmHg) } \\
\text { CBF (units) }\end{array}$ & $2.8 \pm 0$ & $3.0 \pm 0$ & $2.4 \pm 0$ & $2.5 \pm 0$ & 0.48 & 0.82 \\
CVR & $36 \pm 3$ & $35 \pm 4$ & $42 \pm 2$ & $44 \pm 5$ & 0.92 & 0.57 \\
(100*mmHg/units) & $3.0 \pm 0$ & $3.4 \pm 0$ & $2.5 \pm 0$ & $2.7 \pm 0$ & 0.16 & 0.67 \\
CVC (units/mmHg) & &
\end{tabular}

Values are mean \pm SE. MSNA, muscle sympathetic nerve activity $(n=11$ for fish oil and $n=10$ for placebo); FBF, forearm blood flow ( $n=14$ for fish oil and $n=13$ for placebo); FVR, forearm vascular resistance ( $n=14$ for fish oil and $n=13$ for placebo); FVC, forearm vascular conductance ( $n=14$ for fish oil and $n=13$ for placebo); CBF, calf blood flow ( $n=14$ for fish oil and $n=11$ for placebo); CVR, calf vascular resistance $(n=14$ for fish oil and $n=11$ for placebo); CVC, calf vascular conductance ( $n=14$ for fish oil and $n=11$ for placebo). 


\subsubsection{Resting Measurements for Combined Non-Hypertensive Subjects}

We combined normotensive and prehypertensive groups into one nonhypertensive group in order to increase statistical power of our analyses. Combining the normotensive and prehypertensive groups yielded no differences in blood pressure or muscle sympathetic nerve activity responses after fish oil or placebo. Resting values for the combined groups are represented in Table 3.5. There were no significant interactions between fish oil and placebo supplementation groups at rest.

Table 3.5. Baseline values for combined non-hypertensive subjects pre and post fish oil or placebo supplementation.

\begin{tabular}{lcc|cc|c} 
& \multicolumn{2}{c}{ Fish Oil } & \multicolumn{2}{c|}{ Placebo } & Interaction \\
& PRE & POST & PRE & POST & P-Value \\
\hline SAP (mmHg) & $117 \pm 2$ & $115 \pm 2$ & $115 \pm 2$ & $114 \pm 2$ & 0.53 \\
DAP (mmHg) & $67 \pm 1$ & $67 \pm 1$ & $69 \pm 1$ & $69 \pm 1$ & 0.79 \\
MAP (mmHg) & $84 \pm 1$ & $83 \pm 1$ & $84 \pm 1$ & $84 \pm 2$ & 0.67 \\
HR (beats/min) & $72 \pm 2$ & $72 \pm 2$ & $75 \pm 2$ & $75 \pm 2$ & 0.82 \\
MSNA (bursts/min) & $11 \pm 1$ & $10 \pm 1$ & $12 \pm 1$ & $12 \pm 2$ & 0.72 \\
MSNA (bursts/100hb) & $17 \pm 2$ & $16 \pm 2$ & $18 \pm 2$ & $18 \pm 2$ & 0.98 \\
FBF (units) & $3.1 \pm 0.2$ & $3.1 \pm 0.2$ & $2.6 \pm 0.2$ & $2.8 \pm 0.3$ & 0.51 \\
FVR (mmHg/units) & $37 \pm 3$ & $35 \pm 3$ & $42 \pm 3$ & $40 \pm 3$ & 0.86 \\
FVC (100*units/mmHg) & $3.3 \pm 0.3$ & $3.5 \pm 0.3$ & $2.7 \pm 0.2$ & $2.4 \pm 0.3$ & 0.60 \\
CBF (units) & $2.4 \pm 0.2$ & $2.6 \pm 0.2$ & $2.3 \pm 0.1$ & $2.4 \pm 0.2$ & 0.67 \\
CVR (100*mmHg/units) & $46 \pm 3$ & $40 \pm 3$ & $45 \pm 3$ & $42 \pm 3$ & 0.50 \\
CVC (units/mmHg) & $2.5 \pm 0.2$ & $2.9 \pm 0.2$ & $2.4 \pm 0.1$ & $2.8 \pm 0.2^{*}$ & 0.73 \\
\hline Values are mean \pm SE(n & $34.90 \mathrm{fsh}$ &
\end{tabular}

Values are mean \pm SE ( $n=34$ for fish oil, and $n=33$ for placebo unless noted). SAP, systolic arterial pressure; DAP, diastolic arterial pressure; MAP, mean arterial blood pressure; HR, heart rate; MSNA, muscle sympathetic nerve activity ( $n=27$ for fish oil and $n=27$ for placebo); FBF, forearm blood flow ( $n=32$ for fish oil and $n=31$ for placebo); FVR, forearm vascular resistance $(n=32$ for fish oil and $n=31$ for placebo); FVC, forearm vascular conductance $(n=$ 32 for fish oil and $n=31$ for placebo); CBF, calf blood flow ( $n=30$ for fish oil and $n=27$ for placebo); CVR, calf vascular resistance ( $n=30$ for fish oil and $n=27$ for placebo); CVC, calf vascular conductance ( $n=30$ for fish oil and $n=27$ for placebo). 
We further investigated the relationships between blood pressure changes and MSNA changes during rest, and these are presented as figure 3.3 and 3.4. A significant correlation was found between the changes in MAP and MSNA burst frequency following fish oil supplementation $(R=0.354, P=0.03)$ compared to placebo $(R=-0.036, P=0.86)$. Likewise, a correlation was observed when comparing changes in MAP and MSNA burst incidence following fish oil $(R=0.325, P=0.05)$ and placebo $(R=-0.062, P=0.758)$ supplementation. Similarly, a significant change was seen in the change in MSNA bursts per minute $(R=-.466, P=.007)$, and MSNA bursts per 100 heart beats $(R=-.429, P=.013)$ when compared to the resting HR before supplementation.

Normotensive
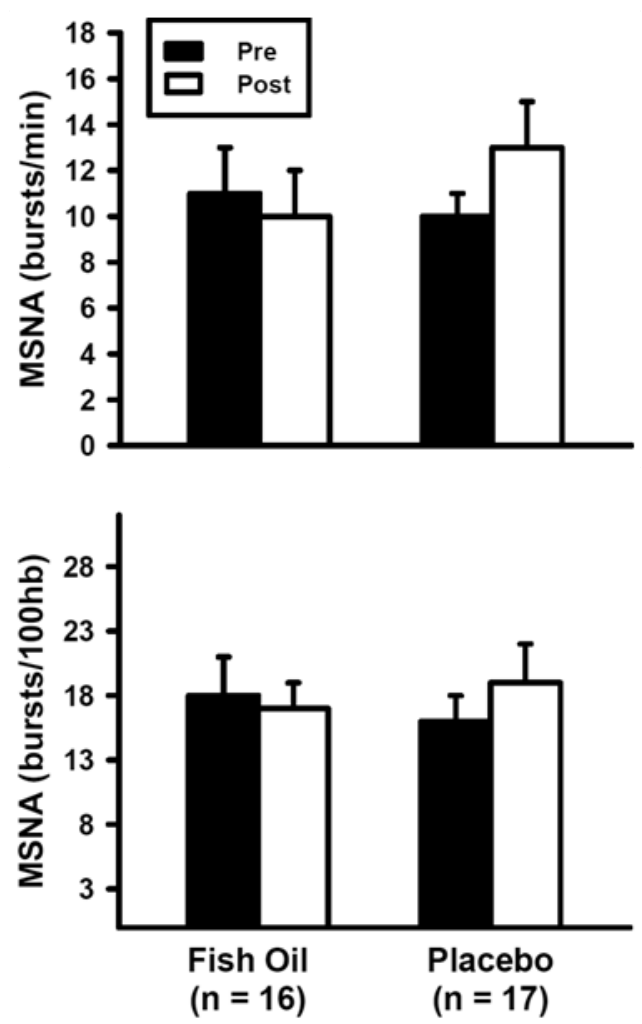

Prehypertensive
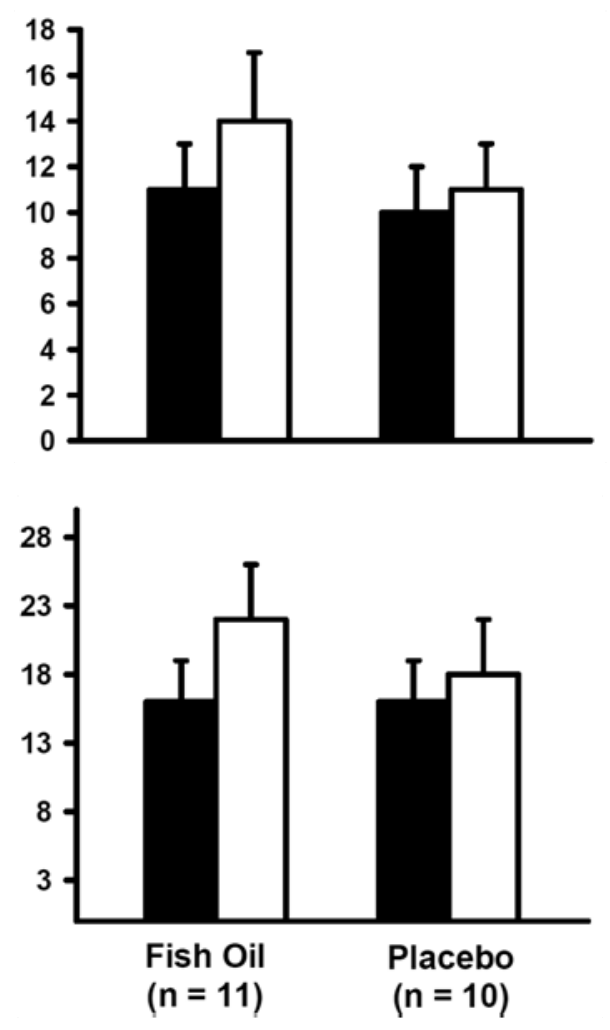

Figure 3.2. Resting MSNA pre and post fish oil and placebo supplementation in normotensive and prehypertensive groups. There was no significance between pre and post treatments. 


\section{Fish Oil}
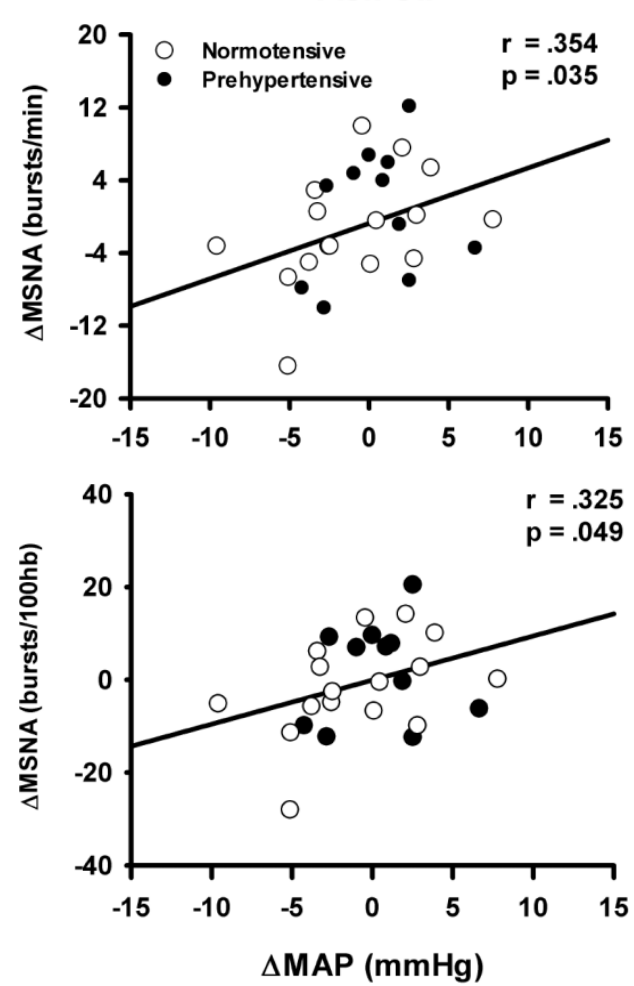

Placebo
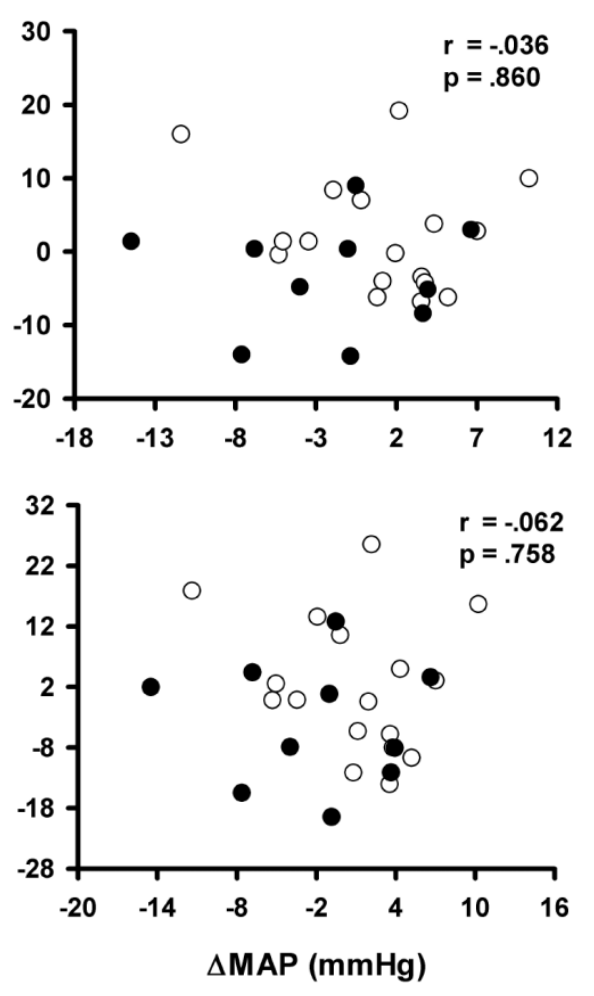

Figure 3.3. Linear regressions of combined non-hypertensive subjects following fish oil or placebo supplementation. Significance was considered as $\mathrm{P}<0.05$

\subsubsection{Hemodynamic Responses to Mental Stress in Prehypertensive and Normotensive}

\section{Subjects}

Blood pressure responses to mental stress in normotensive and prehypertensive subjects are represented in figure 3.4. In normotensives, mental stress significantly increased SAP (fish oil, $\Delta 12 \pm 2$ to $\Delta 13 \pm 2 \mathrm{mmHg}$; placebo, $\Delta 9 \pm 1$ to $\Delta 8 \pm 2 \mathrm{mmHg}$; $\mathrm{P}<.001$ ), DAP (fish oil, $\Delta 10 \pm 1$ to $\Delta 11 \pm 1 \mathrm{mmHg}$; placebo, $\Delta 8 \pm 1$ to $\Delta 8 \pm 1 \mathrm{mmHg}$; $\mathrm{P}<.001$ ), MAP (fish oil, $\Delta 12 \pm 1$ to $\Delta 13 \pm 1 \mathrm{mmHg}$; placebo, $\Delta 10 \pm 1$ to $\Delta 9 \pm 1 \mathrm{mmHg}$; $\mathrm{P}<.001$ ), but these responses were similar across supplementation groups (time $\mathrm{x}$ condition $\mathrm{x}$ drug interactions, $\mathrm{P}>\mathrm{202}$ ). 
FISH OIL
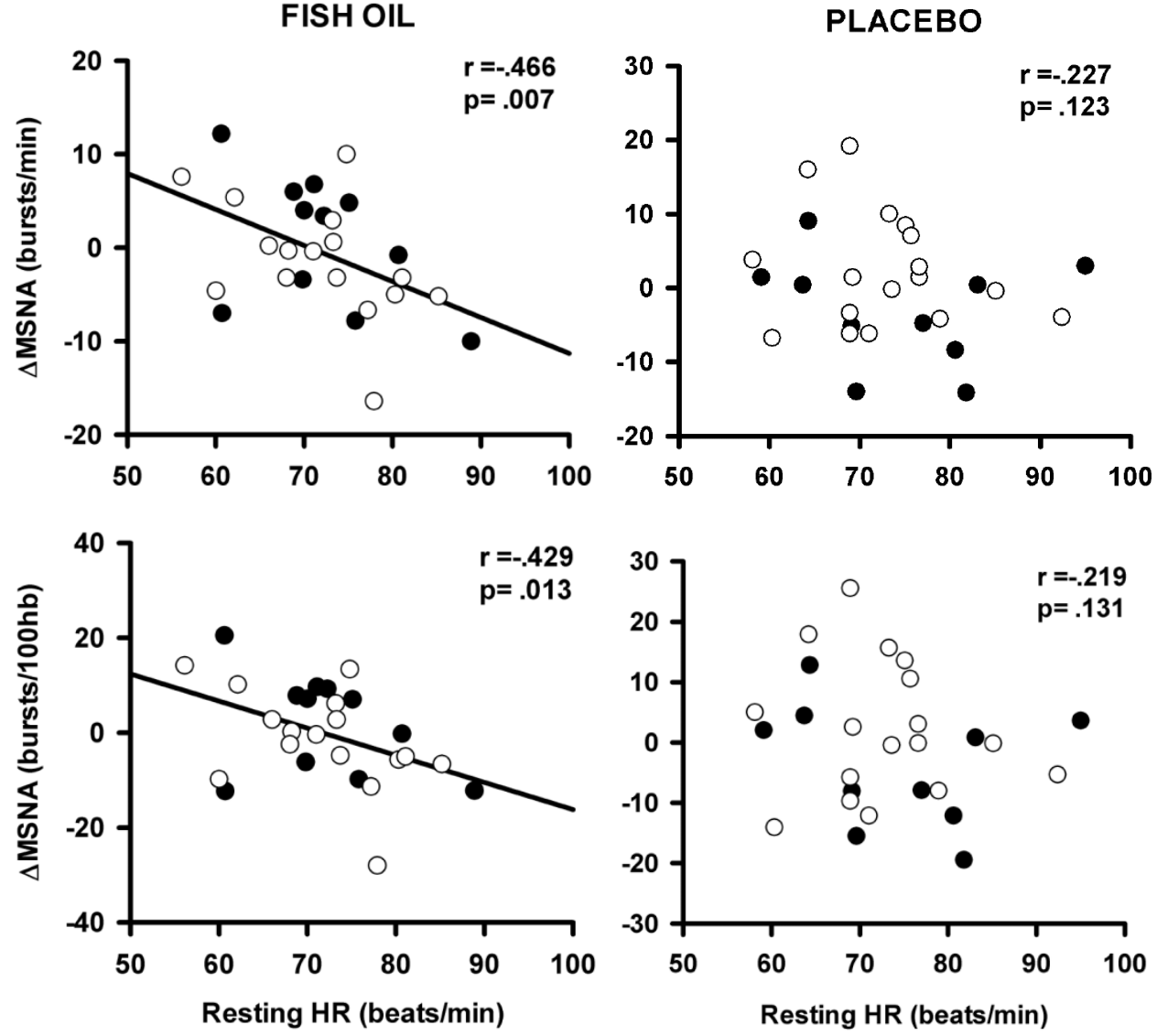

Figure 3.4. Linear regressions between change in MSNA and resting HR following omega-3 supplementation in combined non-hypertensive subjects following fish oil or placebo supplementation. Significance was considered as $\mathrm{P}<0.05$

Similarly in prehypertensives, mental stress significantly increased SAP (fish oil, $\Delta 15 \pm 3$ to $\Delta 14 \pm 3 \mathrm{mmHg}$; placebo, $\Delta 14 \pm 3$ to $\Delta 14 \pm 4 \mathrm{mmHg} ; \mathrm{P}<.001$ ), DAP (fish oil, $\Delta 11 \pm 2$ to $\Delta 10 \pm 1 \mathrm{mmHg}$; placebo, $\Delta 11 \pm 1$ to $\Delta 10 \pm 2 \mathrm{mmHg} ; \mathrm{P}<.001$ ), MAP (fish oil, $\Delta 15 \pm 2$ to $\Delta 13 \pm 2 \mathrm{mmHg}$; placebo, $\Delta 14 \pm 2$ to $\Delta 13 \pm 2 \mathrm{mmHg} ; \mathrm{P}<.001$ ) in both pre and post trials. These responses were similar across supplementation groups (time $x$ condition $x$ drug interactions, $\mathrm{P}>.388)$. 

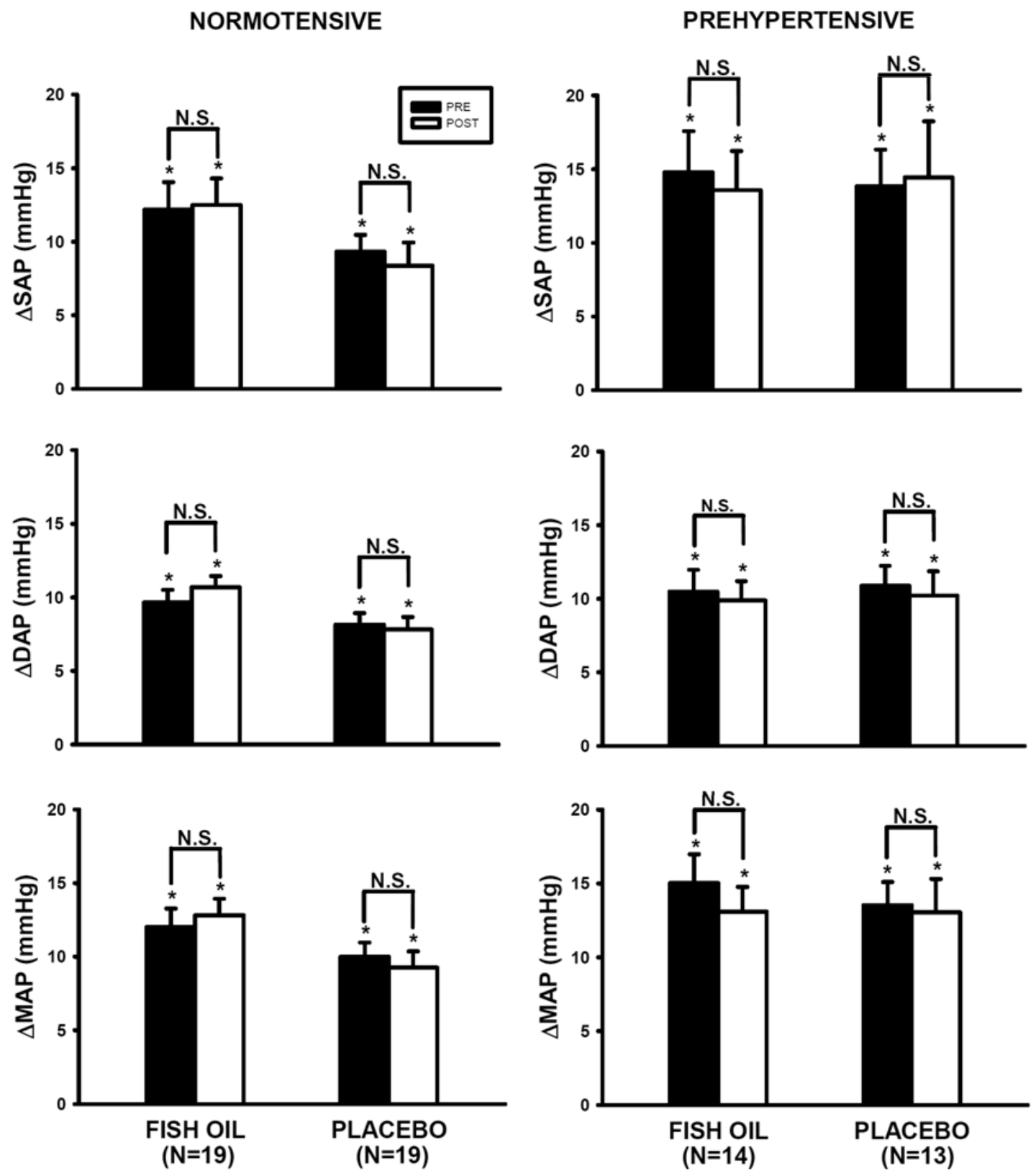

Figure 3.5. Blood pressure responses to mental stress in normotensive and prehypertensive subjects pre and post fish oil or placebo supplementation. *Significantly increased responses during mental stress, $\mathrm{P}<0.05$; N.S., no significance between pre and post trials. 

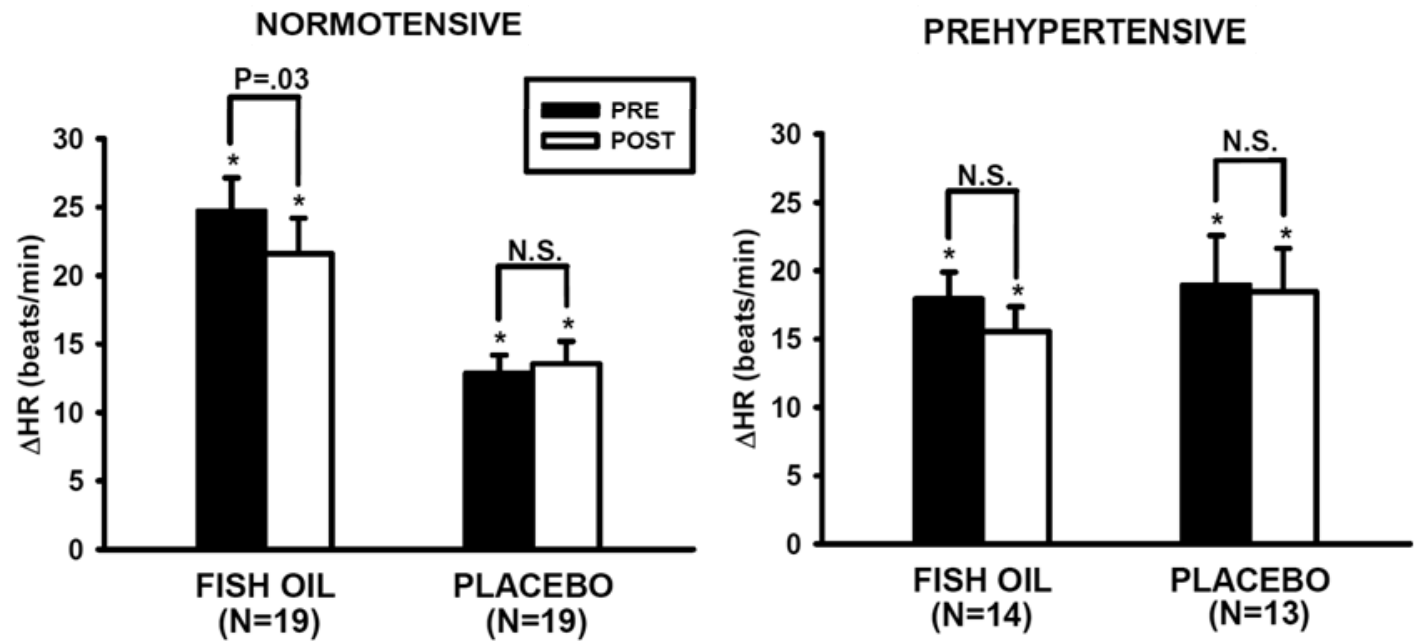

Figure 3.6. Heart rate responses to mental stress in normotensive and prehypertensive subjects pre and post fish oil or placebo supplementation. *Significantly increased responses during mental stress, $\mathrm{P}<0.05$; N.S., no significance between pre and post trials.

Heart rate responses to mental stress are shown in figure 3.6. Mental stress significantly increased $H R$ in normotensives (fish oil, $\Delta 23 \pm 2$ and $\Delta 22 \pm 3$ beats/min; placebo, $\Delta 13 \pm 1$ and $\Delta 14 \pm 2$ beats/min; $P<.001$, pre and post respectively) groups, but this response was blunted post fish oil compared to placebo (time $x$ condition $x$ drug interaction, $\mathrm{P}=.015)$. In prehypertensives, $\mathrm{HR}$ responses to mental stress were significantly increased (fish oil, $\Delta 18 \pm 2$ and $\Delta 16 \pm 2$ beats/min; placebo, $\Delta 19 \pm 4$ and $\Delta 19 \pm 3$ beats/min; $P<.001$, pre and post respectively), but were not significantly different between fish oil and placebo groups (time $x$ condition $x$ drug interaction, $P=.377$ ).

\subsubsection{Sympathetic Nerve Responses to Mental Stress for Normotensive and} Prehypertensive Subjects

MSNA responses to mental stress in normotensive (Table 3.6) and prehypertensive (Table 3.7) subjects are presented below. Neurograms were obtained in only a total of 20 normotensive subjects ( 8 fish oil, 12 placebo), and 9 prehypertensive (4 fish oil, 5 placebo) subjects. This number was not enough to justify statistical analysis due to lack of power for a time $x$ condition $x$ drug comparison. Therefore data are presented in table format. However, review of the data appears to indicate a difference in total MSNA in the fish oil group but not in the placebo group for both normotensive and prehypertensive subjects. Therefore, we pooled the normotensive and 
prehypertensive groups together and analyzed them as a combined group. Data are presented in section 3.3.6.

\subsubsection{Peripheral Vascular Responses to Mental Stress in Prehypertensive and} Normotensive Subjects

Forearm blood flow responses to mental stress in normotensive and prehypertensive subjects are presented in figure 3.7. In normotensives, mental stress elicited statistically similar increases in FBF (fish oil, $\Delta 109.1 \pm 16 \%$ and $\Delta 104.1 \pm 18 \%$; placebo, $\Delta 58.9 \pm 11 \%$ and $\Delta 54.1 \pm 10 \% ; \mathrm{P}<.001$, pre and post respectively), decreases in FVR (fish oil, $\Delta-37.9 \pm 5 \%$ and $\Delta-35.6 \pm 5 \%$; placebo, $\Delta-25.6 \pm 5 \%$ and $\Delta-20.1 \pm 5 \%$; $\mathrm{P}<.001$, pre and post respectively), and increases in FVC (fish oil, $\Delta 86.0 \pm 14 \%$ and $\Delta 78.4 \pm 16 \%$; placebo, $\Delta 45.1 \pm 10 \%$ and $\Delta 39.3 \pm 9 \% ; P<.001$, pre and post respectively). Forearm vascular responses to mental stress were similar in both supplement groups (time $x$ condition $x$ drug interaction, $P>.653$ ).

In prehypertensive subjects, mental stress elicited statistically similar increases in FBF (fish oil, $\Delta 71.8 \pm 14 \%$ and $\Delta 50.4 \pm 13 \%$; placebo, $\Delta 67.6 \pm 17 \%$ and $\Delta 45.6 \pm 15 \%$; $\mathrm{P}<.013$, pre and post respectively), decreases in FVR (fish oil, $\Delta-18.1 \pm 7 \%$ and $\Delta-12.6 \pm$ $8 \%$; placebo, $\Delta-23.6 \pm 7 \%$ and $\Delta-14.1 \pm 6 \%, P<.05$, pre and post respectively), and increases in FVC (fish oil, $\Delta 48.9 \pm 13 \%$ and $\Delta 30.8 \pm 11 \%$; placebo, $\Delta 45.4 \pm 14 \%$ and $\Delta 31.2 \pm 14 \% ; P<.05)$. Similar to normotensive subjects, there were no significant differences between supplement groups (time $x$ condition $x$ drug interaction, $\mathrm{P}>$.697).

Table 3.6. Changes in MSNA during mental stress pre and post fish oil or placebo supplementation in normotensive subjects.

\begin{tabular}{lcc|cc} 
& \multicolumn{2}{c|}{ Fish Oil } & \multicolumn{2}{c}{ Placebo } \\
& PRE & POST & PRE & POST \\
\hline$\Delta$ MSNA (bursts/min) & $6 \pm 2$ & $7 \pm 1$ & $6 \pm 2$ & $7 \pm 2$ \\
$\Delta$ MSNA (bursts/100hb) & $5 \pm 4$ & $6 \pm 2$ & $5 \pm 2$ & $5 \pm 2$ \\
$\Delta$ Total MSNA (arb. units) & $9414 \pm 3145$ & $4944 \pm 849$ & $5547 \pm 1670$ & $7463 \pm 2688$ \\
\hline
\end{tabular}

Values are mean \pm SE. MSNA, muscle sympathetic nerve activity $(n=8$ for fish oil and $n=12$ for placebo); Total MSNA ( $n=7$ for fish oil and $n=10$ for placebo). 
Table 3.7. Changes in MSNA during mental stress pre and post fish oil or placebo supplementation in prehypertensive subjects.

\begin{tabular}{|c|c|c|c|c|}
\hline & \multicolumn{2}{|c|}{ Fish Oil } & \multicolumn{2}{|c|}{ Placebo } \\
\hline & PRE & POST & PRE & POST \\
\hline$\triangle \mathrm{MSNA}$ (bursts/min) & $5 \pm 3$ & $0 \pm 2$ & $6 \pm 2$ & $4 \pm 2$ \\
\hline$\triangle \mathrm{MSNA}$ (bursts/100hb) & $3 \pm 4$ & $-3 \pm 2$ & $1 \pm 2$ & $1 \pm 3$ \\
\hline$\triangle$ Total MSNA (arb. units) & $17349 \pm 6974$ & $-745 \pm 1273$ & $10143 \pm 2405$ & $3402 \pm 2263$ \\
\hline
\end{tabular}

Figure 3.8 represents the calf vascular responses to mental stress in normotensive and prehypertensive subjects. In the normotensive group, mental stress significantly increased CBF (fish oil, $\Delta 53 \pm 12 \%$ and $\Delta 32.9 \pm 8 \%$; placebo $\Delta 25.2 \pm 9 \%$ and $\Delta 22.7 \pm 10 \% ; P<.05$, pre and post respectively). Fish oil groups CVR responses to mental stress were mixed (fish oil, $\Delta-15.1 \pm 7 \%$ pre; $\mathrm{P}<.04$, and $\Delta-6.5 \pm 5 \%$ post; $\mathrm{P}=.243$ ), and placebo CVR responses did not change (fish oil, $\Delta-6.7 \pm 4 \%$ and $\Delta-2.3 \pm$ $6 \% ; \mathrm{P}>.135$, pre and post respectively). CVC significantly increased fish oil $(\Delta 36.3 \pm$ $11 \%$ and $\Delta 16.1 \pm 7 \% ; \mathrm{P}<.05$, pre and post respectively), but not placebo $(\Delta 14.6 \pm 8 \%$ and $\Delta 10.4 \pm 9 \% ; P>.074$, pre and post respectively). Fish oil supplementation blunted CBF $(P=.011)$, CVR $(P=.004)$ and CVC $(P=.007)$ responses to mental stress in fish oil but not placebo.

Prehypertensive calf vascular responses to mental stress were mixed. CBF increased pre and post fish oil and placebo $(\Delta 29.9 \pm 9 \%$ pre, $\Delta 19.1 \pm 7 \%$ post, fish oil; $\Delta 23.4 \pm 7 \%$ pre, $\Delta 23 \pm 8 \%$ post, placebo; $\mathrm{P}<.02$ ), but responses were not different pre vs. post in either group (time $x$ condition $x$ drug interaction, $P>.160$ ). CVR did not change during mental stress $(\Delta 3.1 \pm 9 \%$ pre, $\Delta 3.2 \pm 6 \%$ post, fish oil; $\Delta-0.6 \pm 5 \%$ pre, $\Delta-3.1 \pm 6 \%$ post, placebo; $P=.883$ ), nor was there a difference pre vs. post in either fish oil or placebo group (time $x$ condition $x$ drug interaction, $P=.781$ ). Similarly, CVC did not change during mental stress $(\Delta 12.1 \pm 8 \%$ pre, $\Delta 3.74 \pm 7 \%$ post, fish oil; $\Delta 6.2 \pm 6 \%$ pre, $\Delta 5.8 \pm 5 \%$ post, placebo; $P=.093)$, and these responses were not different when comparing pre vs. post supplement (time $x$ condition $x$ drug interaction, $P=.233$ ). 

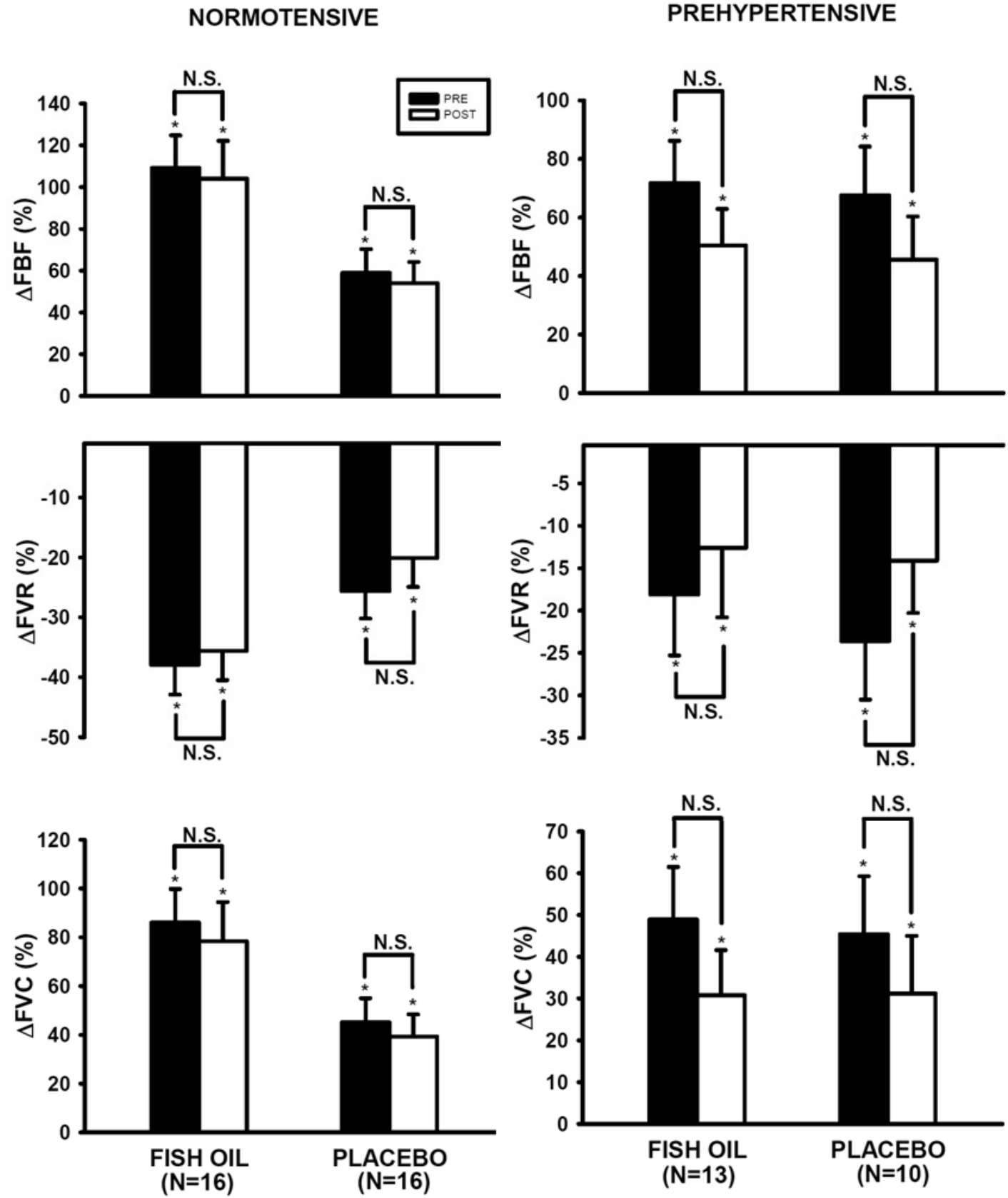

Figure 3.7. Changes $(\Delta)$ in forearm blood flow (FBF), vascular resistance (FVR) and vascular conductance (FVC) during mental stress in normotensive and prehypertensive subjects pre and post fish oil or placebo supplementation. *Significantly increased or decreased responses during mental stress, $\mathrm{P}<0.05$; N.S., no significance between pre and post trials. 

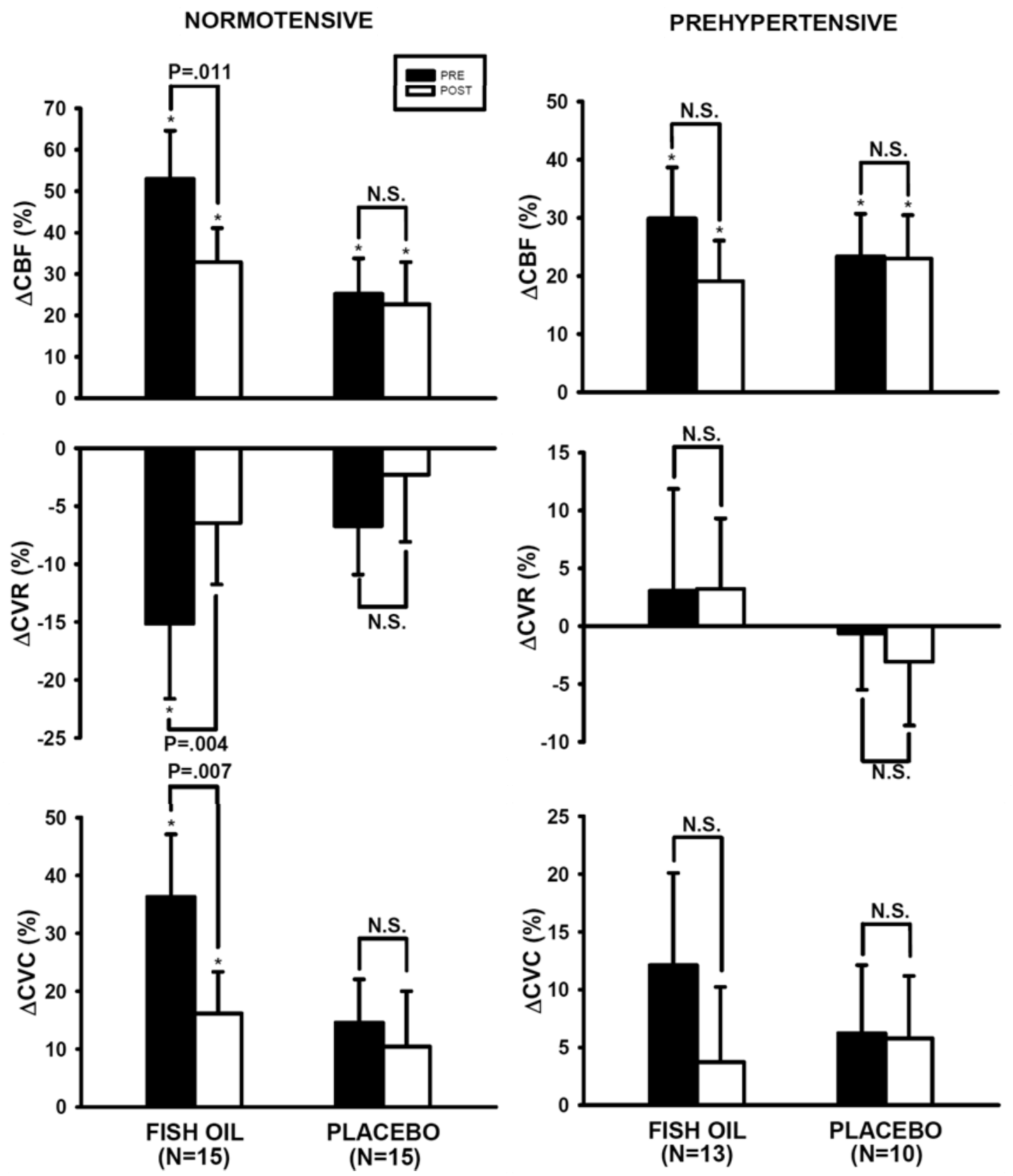

Figure 3.8. Changes $(\Delta)$ in calf blood flow (CBF), vascular resistance (CVR) and vascular conductance (CVC) during mental stress in normotensive and prehypertensive subjects pre and post fish oil or placebo supplementation. *Significantly increase or decrease responses during mental stress, $\mathrm{P}<0.05$; N.S., no significance between pre and post trials. 


\subsubsection{Responses to Mental Stress for Combined Non-Hypertensive Subjects}

Combined blood pressure and heart rate responses to mental stress between fish oil and placebo trials are represented in figure 3.8. Mental stress significantly increased SAP (fish oil, $\Delta 13 \pm 2 \mathrm{mmHg}$ to $\Delta 13 \pm 2 \mathrm{mmHg}$; placebo, $\Delta 11 \pm 1$ to $\Delta 11 \pm 2$ $\mathrm{mmHg}$; $\mathrm{P}<.001$ ), DAP (fish oil, $\Delta 10 \pm 1$ to $\Delta 10 \pm 1 \mathrm{mmHg}$; placebo, $\Delta 9 \pm 1$ to $\Delta 9 \pm 1$ $\mathrm{mmHg} ; \mathrm{P}<.001$ ), and MAP (fish oil, $\Delta 13 \pm 1$ to $\Delta 13 \pm 1 \mathrm{mmHg}$; placebo, $\Delta 11 \pm 1$ to $\Delta 11 \pm$ $1 \mathrm{mmHg} ; \mathrm{P}<.001)$. However, blood pressure responses were not different following fish oil or placebo supplementation (time $x$ condition $x$ drug interaction; $P>340$ ). HR responses to mental stress were attenuated following fish oil supplementation (fish oil, $21.8 \pm 2$ to $\Delta 19.0 \pm 2$ beats $/ \mathrm{min}, \mathrm{P}=.002)$, but not placebo $(\Delta 15.3 \pm 2$ to $\Delta 15.5 \pm 2$ beats/min, $P=.801$ ).

MSNA responses to mental stress are represented in figure 3.9. MSNA burst frequency (fish oil, $\Delta 6 \pm 2$ and $\Delta 5 \pm 1$ bursts/min; placebo, $\Delta 6 \pm 1$ and $\Delta 6 \pm 1$ bursts $/ \mathrm{min}$; $\mathrm{P}<.001$, pre and post respectively) and MSNA burst incidence (fish oil, $\Delta 4 \pm 2$ and $\Delta 3 \pm 3$ bursts/100hb; placebo, $\Delta 4 \pm 2$ and $\Delta 4 \pm 2$ bursts/100hb; $\mathrm{P}<.001$ ) significantly increased. However, there were no differences between supplementation groups (time $\mathrm{x}$ condition $\mathrm{x}$ drug interaction, $P>.700$ ). In contrast, total MSNA (figure 3.10) was reduced post fish oil ( $\Delta 9628 \pm 2407$ to $\Delta 3830 \pm 1029$ a.u., $P=.029$ ), but not in the placebo group ( $\Delta 6860 \pm$ 1463 to $\Delta 6302 \pm 2042$ a.u., $P=.756$ ).

Combined forearm vascular responses to mental stress are shown in figure 3.12. Mental stress increased FBF (fish oil, $\Delta 92 \pm 11 \%$ and $\Delta 80 \pm 11 \% ; \Delta 62 \pm 9 \%$ and $\Delta 51 \pm$ $8 \%$; $\mathrm{P}<.001$, pre and post respectively), FVC (fish oil, $\Delta 69 \pm 10 \%$ and $\Delta 57 \pm 11 \%$;

placebo, $\Delta 45 \pm 8 \%$ and $\Delta 36 \pm 8 \%$; $P<.001$, pre and post respectively), but there were no significant differences between groups (time $\mathrm{x}$ condition $\mathrm{x}$ drug interaction $\mathrm{P}>\mathrm{800}$ ). Similarly, mental stress decreased FVR (fish oil, $\Delta-30 \pm 4 \%$ and $\Delta-25 \pm 5 \%$; placebo, $\Delta$ $25 \pm 4 \%$ and $\Delta-18 \pm 4 \% ; P<.001$, pre and post respectively), but these responses were not significant between groups (time $\mathrm{x}$ condition $\mathrm{x}$ drug interaction $\mathrm{P}=.733$ ). 

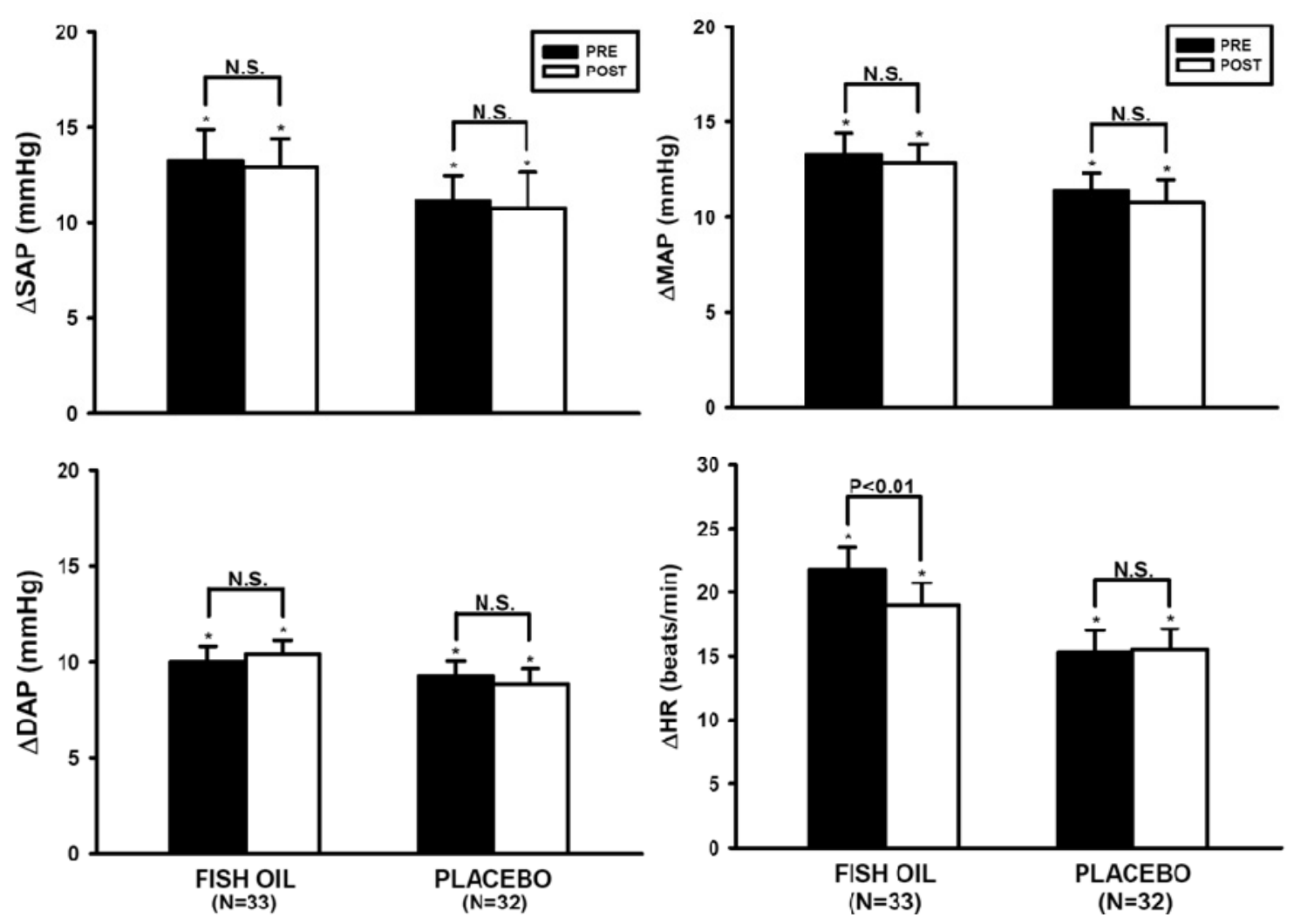

Figure 3.9. Changes $(\Delta)$ in systolic (SAP), diastolic (DAP), mean (MAP) arterial pressures and heart rate (HR) during mental stress in combined normotensive and prehypertensive (nonhypertensive) subjects pre and post fish oil or placebo supplementation. *Significantly increased responses during mental stress, $\mathrm{P}<0.05$; N.S., no significance between pre and post trials.

Combined calf vascular responses to mental stress are shown in figure 3.13. Mental stress increased CBF (fish oil, $\Delta 42 \pm 8 \%$ and $\Delta 27 \pm 6 \%$; placebo, $\Delta 25 \pm 6 \%$ and $\Delta 23 \pm 7 \%$; $P<.002$, pre and post respectively). However, the fish oil group demonstrated a blunted calf vascular response during mental stress compared to placebo (time $x$ condition $x$ drug interaction, $P=.004)$. Mental stress did not change CVR in either the fish oil $(\Delta-7 \pm 6 \%$ and $\Delta-2 \pm 4 \%$ pre and post respectively) or placebo ( $\Delta-4 \pm 3 \%$ and $\Delta-3 \pm$ $4 \%$, pre and post respectively). Mental stress significantly increased CVC in the fish oil group ( $\Delta 25 \pm 7 \%$ and $\Delta 10 \pm 5 \%$; $P<.05$, pre and post respectively), but only during the pre testing in the placebo group $(\Delta 11 \pm 5 \%$ pre, $P<.012, \Delta 10 \pm 6 \%$ post; $P<.147)$. Fish oil significantly blunted the vascular conductance response $(P=.003)$ in the calf compared to no change in the placebo group at rest (time $x$ condition $x$ drug interaction $P=.034$ ). 

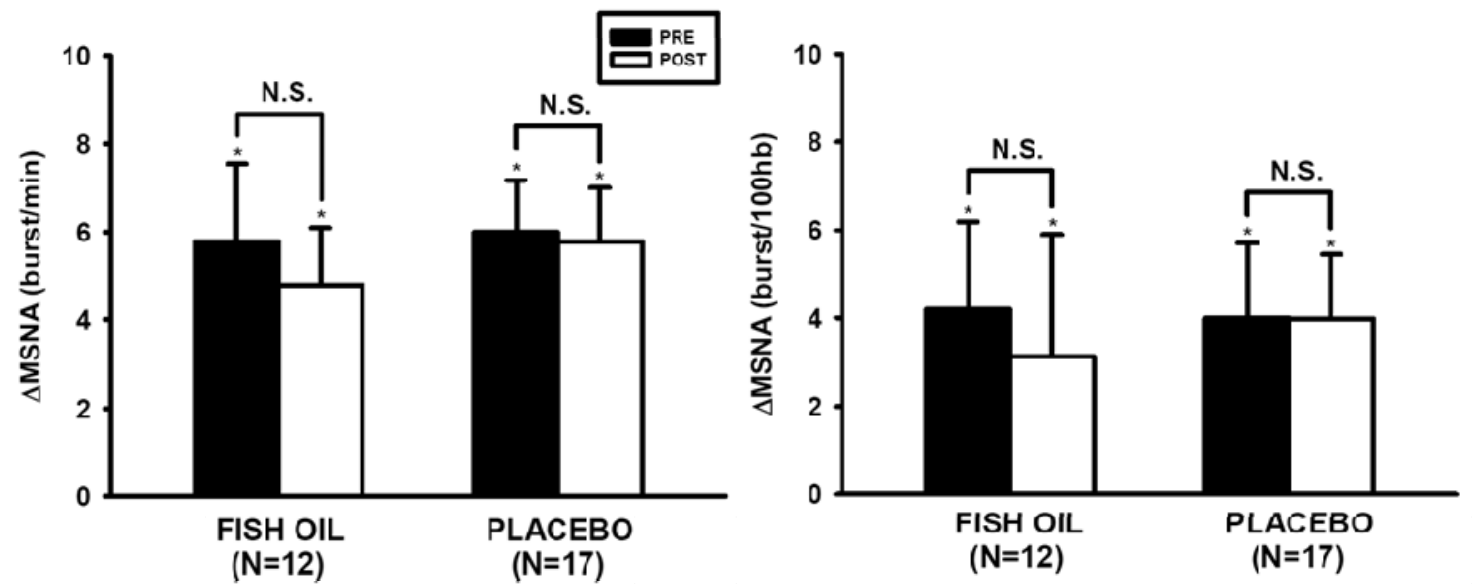

Figure 3.10. Changes $(\Delta)$ in muscle sympathetic nerve activity (MSNA), expressed as bursts per minute and bursts per 100 heartbeats, during mental stress in combined normotensive and prehypertensive (non-hypertensive) subjects pre and post fish oil or placebo supplementation. *Significantly increased responses during mental stress, $P<0.05$; N.S., no significance between pre and post trials.

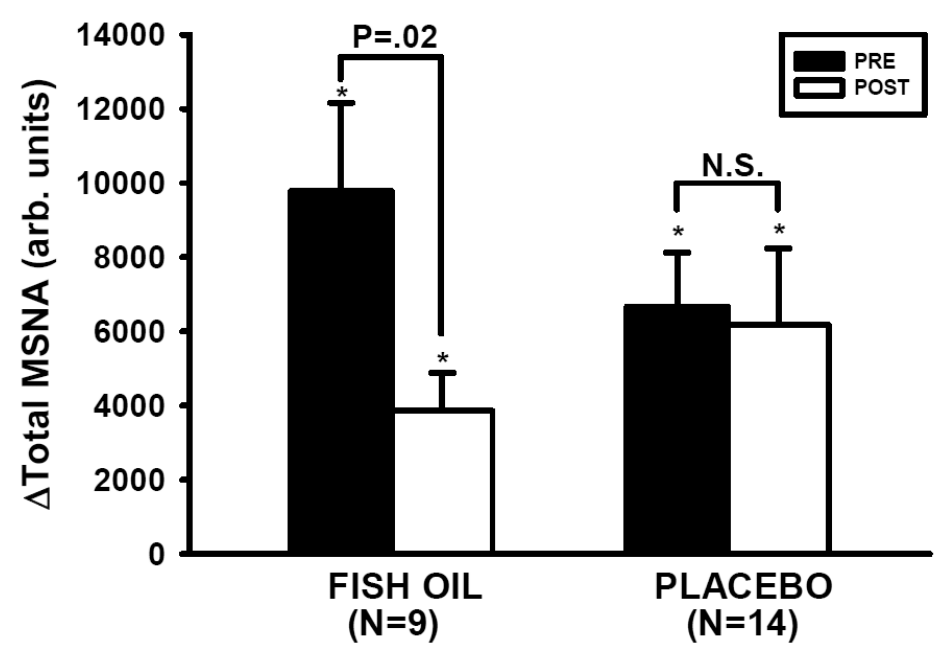

Figure 3.11. Changes $(\Delta)$ in muscle sympathetic nerve activity (MSNA), expressed as total activity, during mental stress in combined normotensive and prehypertensive (non-hypertensive) subjects pre and post fish oil or placebo supplementation. *Significantly increased responses during mental stress, $\mathrm{P}<0.05$; N.S., no significance between pre and post trials. 

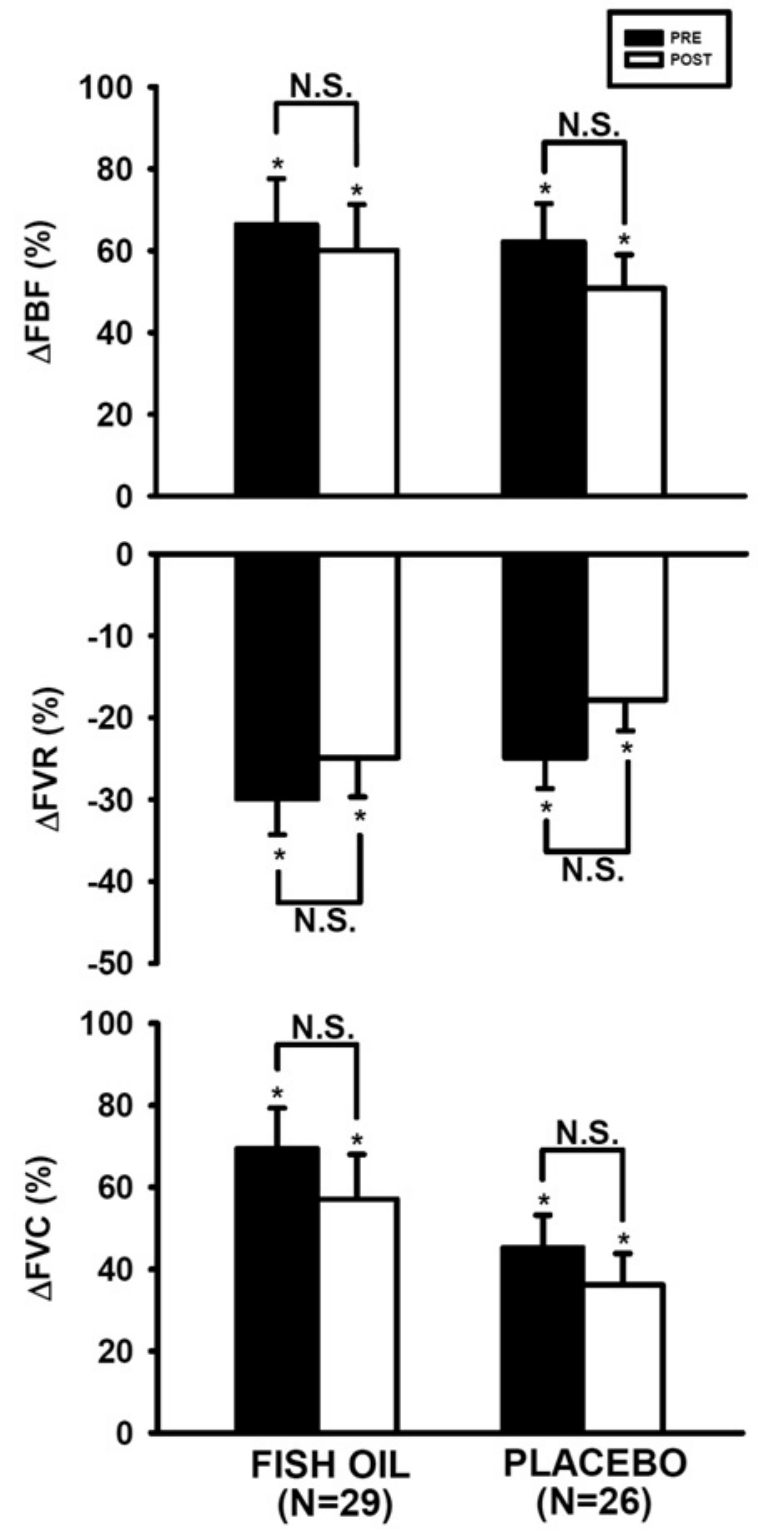

Figure 3.12. Changes $(\Delta)$ in forearm blood flow (FBF), vascular resistance (FVR) and vascular conductance (FVC) during mental stress in combined normotensive and prehypertensive (nonhypertensive) subjects pre and post fish oil or placebo supplementation. *Significantly increased or decreased responses during mental stress, $\mathrm{P}<0.05$; N.S., no significance between pre and post trials. 

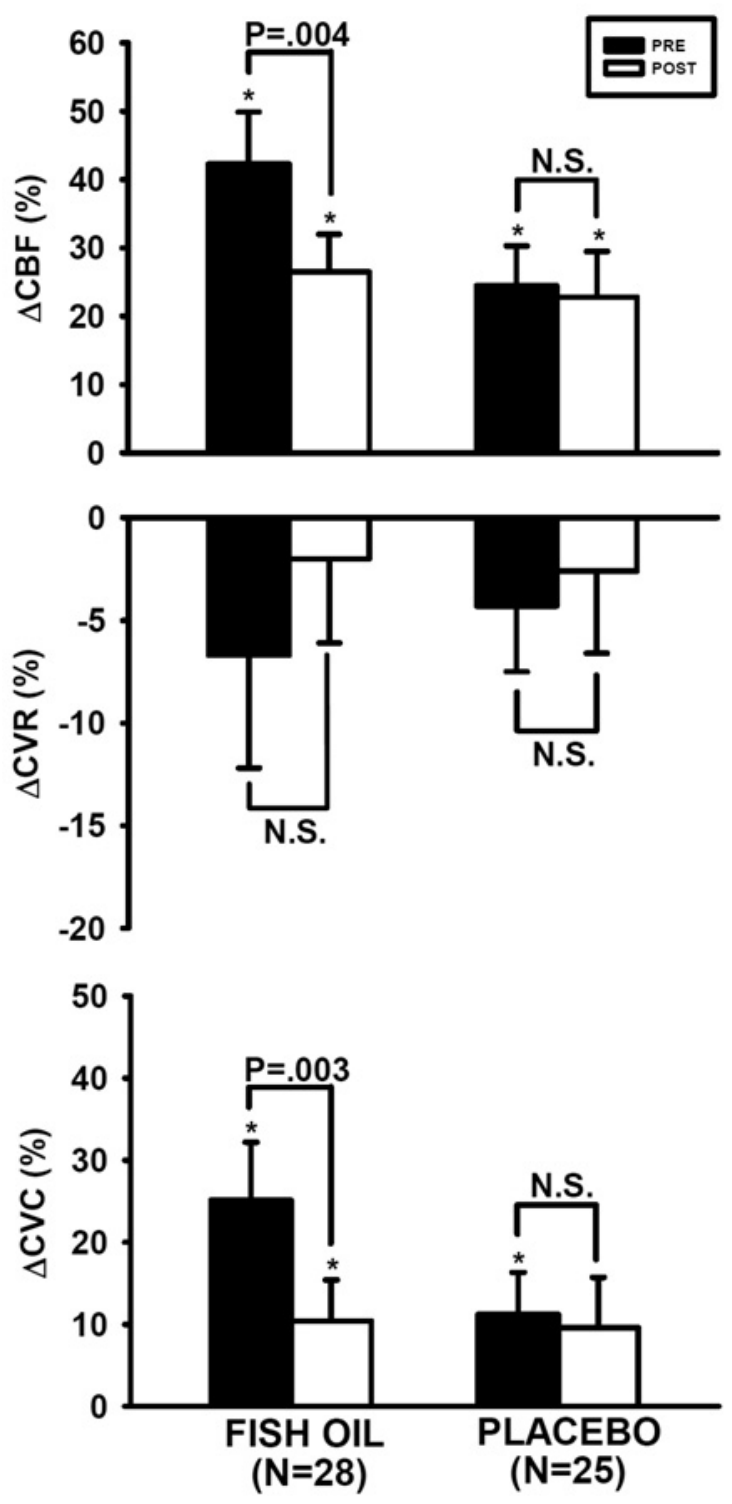

Figure 3.13. Changes $(\Delta)$ in calf blood flow (CBF), vascular resistance (CVR) and vascular conductance (CVC) during mental stress in combined normotensive and prehypertensive (nonhypertensive) subjects pre and post fish oil or placebo supplementation. *Significantly increased or decreased responses during mental stress, $\mathrm{P}<0.05$; N.S., no significance between pre and post trials. 


\subsection{Discussion}

The current investigation offers several new and novel insights into the mechanistic influence of omega-3 fatty acids on neural and cardiovascular function. First, fish oil did not reduce that resting blood pressure or MSNA in either normotensive or prehypertensive individuals. However, further analysis of combined groups revealed a significant correlation between changes in arterial pressure and changes in MSNA following fish oil supplementation. This suggests that fish oil may have an influence on the sympathetic nervous system in individuals who experience a reduction in blood pressure following fish oil supplementation. Second, fish oil attenuated heart rate response during mental stress, and this effect may be mediated by the sympathetic nervous system (i.e., total MSNA). Third, omega-3 supplementation may improve peripheral vascular function at rest, but this does not appear to be specific to fish oils. Combined, these observations offer new information regarding the mechanistic affect omega-3s have on cardiovascular function at rest and during mental stress.

\subsubsection{Resting Neurovascular Responses to Omega-3 supplementation}

Fish oil has been suggested to lower blood pressure in hypertensive rather than normotensive individuals (Appel et al., 1993; Morris et al., 1993). This is the first study to investigate the effect of fish oil in prehypertension. Contrary to our original hypothesis, fish oil supplementation did not reduce resting blood pressure or resting MSNA in prehypertensive individuals. This finding is consistent with a prior study demonstrating no effect of fish oil on resting blood pressure, heart rate or MSNA in healthy normotensive individuals (Monahan et al., 2004). However, we did observe a modest, yet significant, positive correlation between change in sympathetic nerve activity and change in blood pressure in the fish oil group. More specifically, subjects demonstrating a decrease of blood pressure after supplementation demonstrated a concomitant decrease in MSNA. Similarly, we found that a decrease in resting heart rate was significantly correlated with subject's resting blood pressure. It appears that fish oil may have a bradycardic influence as resting arterial pressure increases. Sympathetic nerve activity plays a strong role in both blood pressure regulation and heart rate. Our findings provide new evidence that fish oil may, in part, reduce blood pressure and heart rate via sympathetic neural mechanisms. 
To date, only one study has investigated the effect of fish oil on MSNA. Monahan et al. (2004) reported that fish oil did not alter resting MSNA levels, however the relationship between changes in MSNA and changes in blood pressure or heart rate were not examined. It has been reported that resting heart rate decreases more dramatically in patients with elevated resting heart rates following fish oil supplementation (Mozaffarian et al., 2005). Additionally, EPA and DHA have been reported to lower plasma norepinephrine in healthy volunteers (Hamazaki et al., 2005), and improve heart rate variability measures in older adults (Mozaffarian et al., 2008). These studies support our finding of altered autonomic function as a potential mechanism by which fish oil works to reduce cardiovascular risk. A large scale, longitudinal investigation on cardiovascular disease known as the Framingham study reported that elevated heart rate is linked with a higher incidence of cardiac mortality (Kannel et al., 1987). Additionally, a decrease in heart rate may have significant implications in cardiovascular health (Jouven et al., 2001). We suggest the sympathetic nervous system as a potential mechanism in the reduction of cardiovascular disease from fish oil. However, we recognize this is unlikely to be the only mechanism by which fish oil can play a role in cardiovascular disease prevention.

\subsubsection{Neurovascular Responses to Mental Stress Pre and Post Omega-3 Supplementation}

Fish oil has been suggested to reduce blood pressure through several mechanisms including altered endothelial function, reduced vascular restriction, and changes in the autonomic nervous system (Kannel et al., 1987; Mori \& Woodman, 2006). During mental stress, the classic physiological response is increased heart rate, blood pressure and sympathetic nerve activity with reduced vasoconstriction to the forearm. However the affect of omega-3 fatty acids on these neurovascular mechanisms has not been previously investigated.

Currently, only one study has examined the cardiovascular effects of omega-3 fatty acids during mental stress in humans. Delarue et al. (2003) reported that the circulating plasma catecholamine responses were blunted during mental stress following fish oil supplementation (Delarue et al., 2003). Mental stress is known to simultaneously increase norepinephrine and epinephrine (Esler et al., 1984b). However, epinephrine 
appears to increase more dramatically during psychological stimulation compared to norepinephrine (LeBlanc et al., 1979; Dimsdale \& Moss, 1980). The epinephrine response during mental stress has been linked to sympathetic activation of the adrenal medulla (Reims et al., 2004). We did not analyze circulating catecholamines in the present study. However, MSNA is known to be a robust model of measuring direct sympathetic neural activity. Additionally, it has been demonstrated that MSNA correlates strongly with norepinephrine spillover analysis (Wallin et al., 1992; Kingwell et al., 1994).

Fish oil did not alter MSNA burst frequency or burst incidence responses to mental stress, but did reduce total MSNA. This divergent sympathetic response may be an important distinction in understanding potential mechanisms underlying the effect of fish oil on the sympathetic nervous system during stress. Previously, it has been suggested that sympathetic burst activity and total MSNA operate off of two separate central nervous system (CNS) inputs (Reims et al., 2004). The generation of a burst is suggested to be controlled through a gated (i.e. on/off) mechanism, while the total MSNA appears to operate via graded input. Whereas this gated mechanism may operate off of one CNS input, the graded mechanism may respond to multiple CNS inputs (Kienbaum et al., 2001; Keller et al., 2006). Recently, Keller et al. (2006) demonstrated that whole body heating increased burst activity (i.e. via the gating mechanism), but did not affect the total MSNA response, supporting the theory presented by Kienbaum et al. (2001) that these two mechanisms of muscle sympathetic nerve activity operate on two separate CNS inputs. Using this information, Steinback et al. (2010) began to investigate the neuron recruitment strategies of the sympathetic nerves that cause a greater burst size (i.e. total MSNA). Steinback et al. (2010) showed that the neuron recruitment in muscle sympathetic nerves may follow a similar pattern as the recruitment of skeletal muscle fibers proposed by Henneman et al. (1965). This theory proposes that recruitment of fibers is graded based on size, where the smaller slower fibers are recruited first followed by bigger fibers as greater force or stimulation is required (Henneman et al., 1965; Keller et al., 2006). Collectively, physical stressors have demonstrated different mechanisms in increasing MSNA activity through burst activity (Steinback et al., 2010) or through total MSNA (Keller et al., 2006). Mental stress typically increases both burst activity and total MSNA (Anderson et al., 1991; Callister et al., 1992). Based on the previous findings of Kienbaum et al. (2001), this suggests that 
multiple mechanisms are influencing CNS control over sympathetic nerve activity during mental stress. The current study suggests that fish oil blunts the total MSNA response to mental stress, but not the burst frequency. This infers that fish oil may be affecting sympathetic neuron recruitment more so than a gating mechanism.

The current study demonstrated a blunted heart rate response to mental stress in the fish oil group but not the placebo in combined subjects (i.e., normotensive and prehypertensive groups). There are several possible mechanisms that could contribute to a reduction in heart rate. One such mechanism is sympathetic withdrawal which we have demonstrated through blunted total MSNA. Another mechanism could be an increase in parasympathetic nerve activity. Previous research has suggested that parasympathetic tone increases following fish oil supplementation (Mozaffarian et al., 2008) and it has been suggested that hypertensive individuals have reduced parasympathetic activation on the heart during mental stress (Das, 2000). The only method for indirectly measuring parasympathetic nerve activity is via spectral analysis of heart rate variability. One recent study found that fish oil did not affect HRV during physiological stressors in dogs (Billman \& Harris, 2011). Though we analyzed heart rate variability, the results did not offer any clear insight into the effects of omega 3 fatty acids on parasympathetic activity in normotensive or prehypertensives during mental stress. Therefore the affect of omega- 3 fatty acids on the parasympathetic nervous system during mental stress remains equivocal.

Finally, altered vascular function has also been proposed as a potential mechanism for the cardiovascular benefits associated with fish oil. In the present study we monitored forearm and calf vascular response to mental stress. Whereas the BP, HR and MSNA responses to mental stress between prehypertensive and normotensive (as well as combined subjects) showed a clear picture of the effects of fish oil, the peripheral vascular responses to mental stress post omega-3 supplementation were inconsistent. At rest, the combined non-hypertensive individuals demonstrated increased baseline forearm blood flow, but there was no difference between fish oil and placebo. In contrast to the forearm response, there were no significant changes in calf vascular function at rest. Moreover, vascular responses to mental stress did not change in response to fish oil or placebo. These findings provide inconclusive evidence as to the effects of omega-3 fatty acids on the peripheral vasculature, although it is clear that fish oil and placebo 
appeared to not have any consistent influence on either forearm or calf vascular responses to mental stress. The selective improvement of omega- 3 fatty acids on resting forearm vascular function remains unclear. The evidence that there was no difference between fish oil and placebo might suggest methodological flaws. The current study used olive oil as the placebo treatment, which is an omega-3 fatty acid. Olive oil is commonly used in placebo-based fish oil studies (Monahan et al., 2004), but may have a beneficial effect on vascular function similar to that suggested by fish oil. It has been previously reported that olive oil may contribute to improvement in endothelial function, inflammation, and decrease the risk of cardiovascular disease (Ruediger et al., 2004). To this point, most research using olive oil as a placebo in a fish oil based study have found fish oil to be more effective at lowering blood pressure, and therefore it is commonly used as a placebo. Our findings suggest that olive oil may exert some positive vascular adaptations, thus may not be an ideal placebo. More research comparing olive oil and fish oil appears warranted to establish the true mechanisms behind

In conclusion, we found that fish oil does not consistently decrease resting arterial blood pressure or MSNA in normotensive or prehypertensive humans. However, changes in arterial blood pressure are significantly correlated to changes in MSNA after fish oil, suggesting the sympathetic nervous system does play a role when a fish oilinduced hypotensive response is observed. Moreover, fish oil decreased heart rate and total MSNA responses to mental stress, suggesting fish oil may have positive health benefits regarding neural cardiovascular reactivity in humans. These findings provide new mechanistic insight into nonpharmacological approaches to treating and/or preventing hypertension and other cardiovascular conditions. 


\section{Chapter 4}

\section{Summary and Future Directions}

\subsection{Summary}

Prehypertension is a relatively new classification of blood pressure. This moderate level of blood pressure has been linked to a heightened risk of future development of hypertension (Zhang et al., 2006; Egan \& Julius, 2008). However, the mechanisms responsible for this increased risk are not fully understood. Earlier research investigating borderline hypertensives indicates that the neurovascular system may play a key role in the development of cardiovascular disease (Mark, 1984; Anderson et al., 1989; Santangelo et al., 1989; Matsukawa et al., 1991), and mental stress may potentiate this. However, these mechanisms have not been investigated in prehypertensive patients. It is becoming increasingly important to use preventative measures to decrease the increasing incidence of hypertension and cardiovascular disease. Therefore, we conducted two investigations to further our understanding of the mechanisms contributing to prehypertension (Study 1), and the role of non-pharmacological intervention via fish oil in this same population (Study 2).

In Study 1 we investigated possible mechanisms contributing to prehypertension in young males including sympathetic nerve activity and peripheral vascular responses to mental stress. In Study $\mathbf{2}$ we added omega-3 fatty acids from fish oil to the normal diet of young males and females in order to investigate the mechanisms behind the cardiovascular effects of fish oil on blood pressure and mental stress. We found several novel findings which may lead to a better understanding of prehypertension, hypertension and the role omega- 3 fatty acids have on the cardiovascular system.

From Study 1 we were able to demonstrate that young prehypertensive males experience augmented blood pressure responses to a mental stress task compared to their normotensive counterparts. These responses appear to be linked to a blunted peripheral vasodilation, not an increase in sympathetic nerve activity. Study 2 demonstrated that fish oil had no discernable influence on cardiovascular and nervous system function at rest in prehypertensive or normotensive humans. However, further analysis of the data revealed that fish oil may have had a variable response such that individuals who experienced a decrease in blood pressure also demonstrated a concomitant decrease in MSNA. Moreover, fish oil reduced neural (i.e., total MSNA) and 
cardiovascular (i.e., heart rate) reactivity to mental stress. Robust cardiovascular reactivity has been shown to increase the risk of future development of hypertension (Matthews et al., 1993).

These are the first studies to collectively investigate the neurocardiovascular responses to mental stress both before and after fish oil supplementation in prehypertensive humans. We have several novel findings that further our understanding of prehypertension, hypertension and the possible role that fish oil might play in disease prevention. However, the novel discoveries also introduced more questions that could be answered in future investigations

\subsection{Future Directions - Nitric Oxide}

We have suggested that prehypertensive individuals demonstrate blunted vascular responses to mental stress, and these responses were not mediated through MSNA. Our study only measured whole limb changes blood flow during mental stress. We were unable to investigate other mechanisms that contribute to increased blood flow in the limbs, such as nitric oxide (NO). It has been demonstrated that vascular endothelial cells have the ability to secrete NO as a powerful local vasodilator (Radomski et al., 1987; Shepherd \& Katusic, 1991). The release of NO has been suggested to be mediated by both shear stress on the vascular wall, and by the nervous system, both of which can be affected by hypertension. In humans, mental stress has elicited a NOmediated forearm vasodilatory response (Dietz et al., 1994; Cardillo et al., 1997), and a response that has been blunted hypertensive individuals (Cardillo et al., 1998). Collectively, it is possible that nitric oxide could be playing an important role in the blunted responses we observe in prehypertensive individuals during mental stress. Interestingly, one mechanism by which omega-3 fatty acids has been suggested to lower blood pressure is through nitric oxide mediators (Harris et al., 1997; De Caterina et al., 2000). Future research should investigate the role of nitric oxide in peripheral vasodilation in prehypertension.

\subsection{Conclusion}

This dissertation reports novel and clinically relevant information regarding the prehypertensive population. Furthermore, we have comprehensively investigated the 
role of fish oil plays on neurovascular function. It is clear that there are altered vascular responses to a psychological stress response in prehypertensives, and these responses may be contributing to the further development of hypertension. As public knowledge of prehypertension begins to grow, it will be important to use this classification as an early indicator for preventative care in order to reduce the risks of cardiovascular disease. Fish oil is one current method that is prescribed as a preventative mechanism. We report mixed results regarding the influence of fish oil on neurovascular control in humans. However, our findings suggest a potentially important interaction between fish oil, the sympathetic nervous system, and arterial blood pressure, especially regarding neural and hemodynamic responses to mental stress. 


\section{References}

Abeywardena MY \& Head RJ. (2001). Longchain n-3 polyunsaturated fatty acids and blood vessel function. Cardiovascular Research 52, 361-371.

Anderson EA, Sinkey CA, Lawton WJ \& Mark AL. (1989). Elevated sympathetic nerve activity in borderline hypertensive humans. Evidence from direct intraneural recordings. Hypertension 14, 177-183.

Anderson EA, Sinkey CA \& Mark AL. (1991). Mental stress increases sympathetic nerve activity during sustained baroreceptor stimulation in humans. Hypertension 17, III43-49.

Anderson EA, Wallin BG \& Mark AL. (1987). Dissociation of sympathetic nerve activity in arm and leg muscle during mental stress. Hypertension 9, III114-119.

Appel LJ, Miller ER, Seidler AJ \& Whelton PK. (1993). Does supplementation of diet with 'fish oil' reduce blood pressure? A meta-analysis of controlled clinical trials. Archives of Internal Medicine 153, 1429-1438.

Bang HO, Dyerberg J \& Nielsen AB. (1971). Plasma lipid and lipoprotein pattern in Greenlandic West-coast Eskimos. Lancet 1, 1143-1145.

Bang HO, Dyerberg J \& Sinclair HM. (1980). The composition of the Eskimo food in north western Greenland. American Journal of Clinical Nutrition 33, 2657-2661.

Barcroft H, Brod J, Hejl BZ, Hirsjarvi EA \& Kitchin AH. (1960). The mechanism of the vasodilatation in the forearm muscle during stress (mental arithmetic). Clinical Science 19, 577-586.

Barcroft H \& Edholm OG. (1945). On the vasodilatation in human skeletal muscle during post-haemorrhagic fainting. Journal of Physiology (London) 104, 161-175.

Barcroft J. (1946). The respiratory function of the blood; man under conditions of stress. Journal of the Royal Institute of Public Health 9, 74-85.

Bayorh MA, McGee L \& Feuerstein G. (1989). Acute and chronic effects of eicosapentaenoic acid (EPA) on the cardiovascular system. Research Commununcations in Chemical Patholology and Pharmacology 66, 355-374.

Billman GE \& Harris WS. (2011). Effect of dietary omega-3 fatty acids on the heart rate and the heart rate variability responses to myocardial ischemia or submaximal exercise. American Journal of Physiology - Heart and Circulatory Physiology 300, H2288-2299.

Blair DA, Glover WE, Greenfield AD \& Roddie IC. (1959). Excitation of cholinergic vasodilator nerves to human skeletal muscles during emotional stress. Journal of Physiology (London) 148, 633-647. 
Booth J. (1977). A short history of blood pressure measurement. Proceedings of the Royal Society of Medicine 70, 793-799.

Boutcher YN, Park YJ \& Boutcher SH. (2009). Vascular and baroreceptor abnormalities in young males with a family history of hypertension. European Journal of Applied Physiology 107, 653-658.

Brod J. (1963). Haemodynamic basis of ocute pressor reactions and hypertension. British Heart Journal 25, 227-245.

BROD J, FENCL V, HEJL Z \& JIRKA J. (1959). Circulatory changes underlying blood pressure elevation during acute emotional stress (mental arithmetic) in normotensive and hypertensive subjects. Clinical Science 18, 269-279.

Brown GL \& Gillespie JS. (1957). The output of sympathetic transmitter from the spleen of the cat. Journal of Physiology (London)138, 81-102.

Brundtland GH. (2002). From the World Health Organization. Reducing risks to health, promoting healthy life. JAMA 288, 1974.

Burr ML. (1989). Fish and the cardiovascular system. Progress in Food and Nutrition Science 13, 291-316.

Callister R, Suwarno NO \& Seals DR. (1992). Sympathetic activity is influenced by task difficulty and stress perception during mental challenge in humans. Journal of Physiology (London) 454, 373-387.

Cannon WB. (1914). The Interrelations of Emotions as Suggested by Recent Physiological Researches. The American Journal of Psychology 25, 256-282.

Cannon WB. (1927). Bodily Changes in Pain, Hunger, Fear and Rage. D. Appleton and Company, New York and London.

Cannon WBaR, A. (1933). Studies on Conditions of Activity in Endocrine Organs: Sympathin E and Sympathin I. American Journal of Physiology 104, 557-574.

Cardillo C, Kilcoyne CM, Cannon RO \& Panza JA. (1998). Impairment of the nitric oxidemediated vasodilator response to mental stress in hypertensive but not in hypercholesterolemic patients. Journal of the American College of Cardiology 32, 12071213.

Cardillo C, Kilcoyne CM, Quyyumi AA, Cannon RO \& Panza JA. (1997). Role of nitric oxide in the vasodilator response to mental stress in normal subjects. American Journal of Cardiology 80, 1070-1074.

Carter JR, Kupiers NT \& Ray CA. (2005). Neurovascular responses to mental stress. Journal of Physiology (London) 564, 321-327. 
Carter JR \& Ray CA. (2009). Sympathetic neural responses to mental stress: responders, nonresponders and sex differences. American Journal of Physiology - Heart and Circulatory Physiology 296, H847-853.

Chin-Dusting JP, Jovanovska V, Kingwell BA, Du XJ \& Dart AM. (1998). Effect of fish oil supplementation on aortic compliance in rats: role of the endothelium. Prostaglandins Leukotrienes and Essential Fatty Acids 59, 335-340.

Chobanian AV, Bakris GL, Black HR, Cushman WC, Green LA, Izzo JL, Jr., Jones DW, Materson BJ, Oparil S, Wright JT, Jr. \& Roccella EJ. (2003). Seventh report of the Joint National Committee on Prevention, Detection, Evaluation, and Treatment of High Blood Pressure. Hypertension 42, 1206-1252.

Collins R, Peto R, MacMahon S, Hebert P, Fiebach NH, Eberlein KA, Godwin J, Qizilbash N, Taylor JO \& Hennekens CH. (1990). Blood pressure, stroke, and coronary heart disease. Part 2, Short-term reductions in blood pressure: overview of randomised drug trials in their epidemiological context. Lancet 335, 827-838.

Cushman WC, Ford CE, Cutler JA, Margolis KL, Davis BR, Grimm RH, Black HR, Hamilton BP, Holland J, Nwachuku C, Papademetriou V, Probstfield J, Wright JT, Jr., Alderman MH, Weiss RJ, Piller L, Bettencourt J \& Walsh SM. (2002). Success and predictors of blood pressure control in diverse North American settings: the antihypertensive and lipid-lowering treatment to prevent heart attack trial (ALLHAT). Journal of Clinical Hypertension (Greenwich) 4, 393-404.

Darwin C. (1886). The Expression of the Emotions in Man and Animals. D. Appleton and Company, New York, NY.

Das UN. (2000). Beneficial effect(s) of n-3 fatty acids in cardiovascular diseases: but, why and how? Prostaglandins Leukotrienes and Essential Fatty Acids 63, 351-362.

De Caterina R, Bernini W, Carluccio MA, Liao JK \& Libby P. (1998). Structural requirements for inhibition of cytokine-induced endothelial activation by unsaturated fatty acids. Journal of Lipid Research 39, 1062-1070.

De Caterina R, Liao JK \& Libby P. (2000). Fatty acid modulation of endothelial activation. American Journal of Clinical Nutrition 71, 213S-223S.

Deanfield JE, Shea M, Kensett M, Horlock P, Wilson RA, de Landsheere CM \& Selwyn AP. (1984). Silent myocardial ischaemia due to mental stress. Lancet 2, 1001-1005.

Delarue J, Matzinger O, Binnert C, Schneiter P, Chiolero R \& Tappy L. (2003). Fish oil prevents the adrenal activation elicited by mental stress in healthy men. Diabetes and Metabolism 29, 289-295.

Delius W, Hagbarth KE, Hongell A \& Wallin BG. (1972). Manoeuvres affecting sympathetic outflow in human muscle nerves. Acta Physiologica Scandinavica 84, 8294. 
Delius W, Wallin G \& Hagbarth KE. (1973). Role of sympathetic nerve impulses in regulation of peripheral circulation. Scandinavian Journal of Clinical and Laboratory Investigation. Supplement 128, 47-50.

Dietz NM, Rivera JM, Eggener SE, Fix RT, Warner DO \& Joyner MJ. (1994). Nitric oxide contributes to the rise in forearm blood flow during mental stress in humans. Journal of Physiology (London) 480 ( Pt 2), 361-368.

Dimsdale JE \& Moss J. (1980). Plasma catecholamines in stress and exercise. JAMA 243, 340-342.

Durocher JJ, Klein JC \& Carter JR. (2011). Attenuation of sympathetic baroreflex sensitivity during the onset of acute mental stress in humans. American Journal of Physiology - Heart and Circulatory Physiology 300, H1788-1793.

Eckberg DL. (1997). Sympathovagal balance: a critical appraisal. Circulation 96, 32243232.

Egan B, Panis R, Hinderliter A, Schork N \& Julius S. (1987). Mechanism of increased alpha adrenergic vasoconstriction in human essential hypertension. Journal of Clinical Investigation 80, 812-817.

Egan BM \& Julius S. (2008). Prehypertension: risk stratification and management considerations. Current Hypertension Reports 10, 359-366.

Eliasson K. (1985). Borderline hypertension. Circulatory, sympatho-adrenal and psychological reactions to stress. Acta Med Scand Supp/ 692, 1-90.

Endres S, Ghorbani R, Kelley VE, Georgilis K, Lonnemann G, van der Meer JW, Cannon JG, Rogers TS, Klempner MS, Weber PC \& et al. (1989). The effect of dietary supplementation with n-3 polyunsaturated fatty acids on the synthesis of interleukin-1 and tumor necrosis factor by mononuclear cells. New England Journal of Medicine 320, 265-271.

Engelman K \& Portnoy B. (1970). A sensitive double-isotope derivative assay for norepinephrine and epinephrine. Normal resting human plasma levels. Circulation Research 26, 53-57.

Epstein BJ. (2010). Improving blood pressure control rates by optimizing combination antihypertensive therapy. Expert Opinions in Pharmacotherapy 11, 2011-2026.

Esler M, Eikelis N, Schlaich M, Lambert G, Alvarenga M, Dawood T, Kaye D, Barton D, Pier C, Guo L, Brenchley C, Jennings G \& Lambert E. (2008). Chronic mental stress is a cause of essential hypertension: presence of biological markers of stress. Clinical and Experimental Pharmacology and Physiology 35, 498-502.

Esler M, Jackman G, Bobik A, Kelleher D, Jennings G, Leonard P, Skews H \& Korner P. (1979). Determination of norepinephrine apparent release rate and clearance in humans. Life Sciences 25, 1461-1470. 
Esler M, Jennings G, Biviano B, Lambert G \& Hasking G. (1986). Mechanism of elevated plasma noradrenaline in the course of essential hypertension. Journal of Cardiovascular Pharmacology 8 Suppl 5, S39-43.

Esler M, Jennings G, Korner P, Blombery P, Sacharias N \& Leonard P. (1984a). Measurement of total and organ-specific norepinephrine kinetics in humans. American Journal of Physiology 247, E21-28.

Esler M, Jennings G \& Lambert G. (1989). Measurement of overall and cardiac norepinephrine release into plasma during cognitive challenge.

Psychoneuroendocrinology 14, 477-481.

Esler M, Lambert G, Brunner-La Rocca HP, Vaddadi G \& Kaye D. (2003). Sympathetic nerve activity and neurotransmitter release in humans: translation from pathophysiology into clinical practice. Acta Physiologica Scandinavica 177, 275-284.

Esler MD, Jennings GL, Johns J, Burke F, Little PJ \& Leonard P. (1984b). Estimation of 'total' renal, cardiac and splanchnic sympathetic nervous tone in essential hypertension from measurements of noradrenaline release. Journal of Hypertension Supplement 2, S123-125.

Esler MD \& Nestel PJ. (1973). High catecholamine essential hypertension: clinical and physiological characteristics. Australian and New Zealand Journal of Medicine 3, 117123.

Esunge PM. (1991). From blood pressure to hypertension: the history of research. Journal of the Royal Society of Medicine 84, 621.

Falkner B, Onesti G, Angelakos ET, Fernandes M \& Langman C. (1979). Cardiovascular response to mental stress in normal adolescents with hypertensive parents.

Hemodynamics and mental stress in adolescents. Hypertension 1, 23-30.

FitzGerald GA, Hossmann V \& Dollery CT. (1981). Norepinephrine release in essential hypertension. Clinical Pharmacology and Therapeutics 30, 164-171.

Fuchs FD. (2010). Prehypertension: the rationale for early drug therapy.

Cardiovascular Therapy 28, 339-343.

Geleijnse JM, Giltay EJ, Grobbee DE, Donders AR \& Kok FJ. (2002). Blood pressure response to fish oil supplementation: metaregression analysis of randomized trials. Journal Hypertension 20, 1493-1499.

Gibbons GH. (1997). Endothelial function as a determinant of vascular function and structure: a new therapeutic target. American Journal of Cardiology 79, 3-8.

Goldstein DS. (1983). Arterial baroreflex sensitivity, plasma catecholamines, and pressor responsiveness in essential hypertension. Circulation 68, 234-240. 
Goldstein DS, Eisenhofer G, Sax FL, Keiser HR \& Kopin IJ. (1987). Plasma norepinephrine pharmacokinetics during mental challenge. Psychosomatic Medicine 49, 591-605.

Grassi G \& Esler M. (1999). How to assess sympathetic activity in humans. Journal of Hypertension 17, 719-734.

Hagbarth KE \& Vallbo AB. (1967). Mechanoreceptor activity recorded percutaneously with semi-microelectrodes in human peripheral nerves. Acta Physiologica Scandinavica $69,121-122$.

Hagbarth KE \& Vallbo AB. (1969). Single unit recordings from muscle nerves in human subjects. Acta Physiologica Scandinavica 76, 321-334.

Halliwill JR, Lawler LA, Eickhoff TJ, Dietz NM, Nauss LA \& Joyner MJ. (1997). Forearm sympathetic withdrawal and vasodilatation during mental stress in humans. Journal of Physiology (London) 504 ( Pt 1), 211-220.

Hamazaki K, Itomura M, Huan M, Nishizawa H, Sawazaki S, Tanouchi M, Watanabe S, Hamazaki T, Terasawa K \& Yazawa K. (2005). Effect of omega-3 fatty acid-containing phospholipids on blood catecholamine concentrations in healthy volunteers: a randomized, placebo-controlled, double-blind trial. Nutrition 21, 705-710.

Harris WS, Rambjor GS, Windsor SL \& Diederich D. (1997). n-3 fatty acids and urinary excretion of nitric oxide metabolites in humans. American Journal of Clinical Nutrition 65, 459-464.

Harvey W. (1628). On the Motion of the Heart and Blood in Animals. P.F. Collier \& Son, 1909-14, New York. Published online in 2001, www.bartelby.com/38/3.

Hashimoto M, Hossain S, Yamasaki H, Yazawa K \& Masumura S. (1999). Effects of eicosapentaenoic acid and docosahexaenoic acid on plasma membrane fluidity of aortic endothelial cells. Lipids 34, 1297-1304.

Heidenreich PA, Trogdon JG, Khavjou OA, Butler J, Dracup K, Ezekowitz MD, Finkelstein EA, Hong Y, Johnston SC, Khera A, Lloyd-Jones DM, Nelson SA, Nichol G, Orenstein D, Wilson PW \& Woo YJ. (2011). Forecasting the future of cardiovascular disease in the United States: a policy statement from the American Heart Association. Circulation 123, 933-944.

Henneman E, Somjen G \& Carpenter DO. (1965). Excitability and inhibitability of motoneurons of different sizes. J Neurophysiol 28, 599-620.

Herd JA. (1991). Cardiovascular response to stress. Physiological Reviews 71, 305-330.

Hertting G. (1964). The Fate of Norepinephrine (Ne) at the Sympathetic Nerve Endings. Acta Neurovegetativa 26, 267-270. 
Heymans C. (1958). Baroceptor and chemoceptor reflexes in monkeys. Circulation Research 6, 567-569.

Hjemdahl P. (1984). Inter-laboratory comparison of plasma catecholamine determinations using several different assays. Acta Physiologica Scandinavica. Supplementum 527, 43-54.

Hjemdahl P, Fagius J, Freyschuss U, Wallin BG, Daleskog M, Bohlin G \& Perski A. (1989). Muscle sympathetic activity and norepinephrine release during mental challenge in humans. American Journal of Physiology 257, E654-664.

Hjemdahl P, Freyschuss U, Juhlin-Dannfelt A \& Linde B. (1984). Differentiated sympathetic activation during mental stress evoked by the Stroop test. Acta Physiologica Scandinavica. Supplementum 527, 25-29.

Howe PR. (1995). Can we recommend fish oil for hypertension? Clinical and Experimental Pharmacology and Physiology 22, 199-203.

Howe PR, Lungershausen YK, Rogers PF, Gerkens JF, Head RJ \& Smith RM. (1991). Effects of dietary sodium and fish oil on blood pressure development in stroke-prone spontaneously hypertensive rats. Journal of Hypertension 9, 639-644.

Huang JL, Chiou CW, Ting CT, Chen YT \& Chen SA. (2001). Sudden changes in heart rate variability during the 1999 Taiwan earthquake. American Journal of Cardiology 87, 245-248, A249.

Izzo JL, Jr. (2007). Prehypertension: demographics, pathophysiology, and treatment. Current Hypertension Reports 9, 264-268.

Jern S, Bergbrant A, Hedner T \& Hansson L. (1995). Enhanced pressor responses to experimental and daily-life stress in borderline hypertension. Journal of Hypertension 13, 69-79.

Jones PP, Spraul M, Matt KS, Seals DR, Skinner JS \& Ravussin E. (1996). Gender does not influence sympathetic neural reactivity to stress in healthy humans. American Journal of Physiology 270, H350-357.

Jouven X, Zureik M, Desnos M, Guerot C \& Ducimetiere P. (2001). Resting heart rate as a predictive risk factor for sudden death in middle-aged men. Cardiovascular Research 50, 373-378.

Joyner MJ, Charkoudian N \& Wallin BG. (2010). Sympathetic nervous system and blood pressure in humans: individualized patterns of regulation and their implications. Hypertension 56, 10-16.

Kannel WB. (1975). Role of blood pressure in cardiovascular disease: the Framingham Study. Angiology 26, 1-14. 
Kannel WB, Kannel C, Paffenbarger RS \& Cupples LA. (1987). Heart rate and cardiovascular mortality: the Framingham Study. American Heart Journal 113, 14891494.

Keith NM, Wagener HP \& Barker NW. (1974). Some different types of essential hypertension: their course and prognosis. American Journal of the Medical Sciences 268, 336-345.

Keller DM, Cui J, Davis SL, Low DA \& Crandall CG. (2006). Heat stress enhances arterial baroreflex control of muscle sympathetic nerve activity via increased sensitivity of burst gating, not burst area, in humans. Journal of Physiology (London) 573, 445-451.

Kenny D, Warltier DC, Pleuss JA, Hoffmann RG, Goodfriend TL \& Egan BM. (1992). Effect of omega-3 fatty acids on the vascular response to angiotensin in normotensive men. American Journal of Cardiology 70, 1347-1352.

Khan F, Elherik K, Bolton-Smith C, Barr R, Hill A, Murrie I \& Belch JJ. (2003). The effects of dietary fatty acid supplementation on endothelial function and vascular tone in healthy subjects. Cardiovascular Research 59, 955-962.

Kienbaum P, Karlssonn T, Sverrisdottir YB, Elam M \& Wallin BG. (2001). Two sites for modulation of human sympathetic activity by arterial baroreceptors? Journal of Physiology (London) 531, 861-869.

Kingwell BA, Thompson JM, Kaye DM, McPherson GA, Jennings GL \& Esler MD. (1994). Heart rate spectral analysis, cardiac norepinephrine spillover, and muscle sympathetic nerve activity during human sympathetic nervous activation and failure. Circulation 90, 234-240.

Knapp HR \& FitzGerald GA. (1989). The antihypertensive effects of fish oil. A controlled study of polyunsaturated fatty acid supplements in essential hypertension. New England Journal of Medicine 320, 1037-1043.

Kris-Etherton PM, Harris WS \& Appel LJ. (2003). Fish consumption, fish oil, omega-3 fatty acids, and cardiovascular disease. Arteriosclerosis, Thrombosis, and Vascular Biology 23, e20-30.

Kuipers NT, Sauder CL, Carter JR \& Ray CA. (2008). Neurovascular responses to mental stress in the supine and upright postures. Journal of Applied Physiology 104, 1129-1136.

Lawrence JE, Klein JC \& Carter JR. (2010). Menstrual cycle elicits divergent forearm vascular responses to vestibular activation in humans. Autonomic Neuroscience 154, 89-93.

LeBlanc J, Côté J, Jobin M \& Labrie A. (1979). Plasma catecholamines and cardiovascular responses to cold and mental activity. Journal of Applied Physiology 47, 1207-1211. 
Leimbach WN, Jr., Wallin BG, Victor RG, Aylward PE, Sundlof G \& Mark AL. (1986). Direct evidence from intraneural recordings for increased central sympathetic outflow in patients with heart failure. Circulation 73, 913-919.

Leor J, Poole WK \& Kloner RA. (1996). Sudden cardiac death triggered by an earthquake. New England Journal of Medicine 334, 413-419.

Levinson PD, losiphidis AH, Saritelli AL, Herbert PN \& Steiner M. (1990). Effects of n-3 fatty acids in essential hypertension. American Journal of Hypertension 3, 754-760.

Lindqvist M, Davidsson S, Hjemdahl P \& Melcher A. (1996). Sustained forearm vasodilation in humans during mental stress is not neurogenically mediated. Acta Physiologica Scandinavica 158, 7-14.

Lindqvist M, Kahan T, Melcher A \& Hjemdahl P. (1993). Cardiovascular and sympathoadrenal responses to mental stress in primary hypertension. Clinical Science (London) 85, 401-409.

Lynch J, Krause N, Kaplan GA, Salonen R \& Salonen JT. (1997). Workplace demands, economic reward, and progression of carotid atherosclerosis. Circulation 96, 302-307.

MacMahon S, Peto R, Cutler J, Collins R, Sorlie P, Neaton J, Abbott R, Godwin J, Dyer A \& Stamler J. (1990). Blood pressure, stroke, and coronary heart disease. Part 1, Prolonged differences in blood pressure: prospective observational studies corrected for the regression dilution bias. Lancet 335, 765-774.

Malliani A, Pagani M, Lombardi F, Furlan R, Guzzetti S \& Cerutti S. (1991). Spectral analysis to assess increased sympathetic tone in arterial hypertension. Hypertension 17, III36-42.

Malmo RB \& Shagass C. (1949). Variability of heart rate in relation to age, sex and stress. Journal of Applied Physiology 2, 181-184.

Marchioli R, Barzi F, Bomba E, Chieffo C, Di Gregorio D, Di Mascio R, Franzosi MG, Geraci E, Levantesi G, Maggioni AP, Mantini L, Marfisi RM, Mastrogiuseppe G, Mininni N, Nicolosi GL, Santini M, Schweiger C, Tavazzi L, Tognoni G, Tucci C \& Valagussa F. (2002). Early protection against sudden death by n-3 polyunsaturated fatty acids after myocardial infarction: time-course analysis of the results of the Gruppo Italiano per lo Studio della Sopravvivenza nell'Infarto Miocardico (GISSI)-Prevenzione. Circulation 105, 1897-1903.

Mark AL. (1984). Structural changes in resistance and capacitance vessels in borderline hypertension. Hypertension 6, III69-73.

Matsukawa T, Gotoh E, Uneda S, Miyajima E, Shionoiri H, Tochikubo O \& Ishii M. (1991). Augmented sympathetic nerve activity in response to stressors in young borderline hypertensive men. Acta Physiologica Scandinavica 141, 157-165. 
Matthews KA, Katholi CR, McCreath $\mathrm{H}$, Whooley MA, Williams DR, Zhu S \& Markovitz $\mathrm{JH}$. (2004). Blood pressure reactivity to psychological stress predicts hypertension in the CARDIA study. Circulation 110, 74-78.

Matthews KA, Woodall KL \& Allen MT. (1993). Cardiovascular reactivity to stress predicts future blood pressure status. Hypertension 22, 479-485.

Monahan KD \& Ray CA. (2002). Limb neurovascular control during altered otolithic input in humans. Journal of Physiology (London) 538, 303-308.

Monahan KD, Wilson TE \& Ray CA. (2004). Omega-3 fatty acid supplementation augments sympathetic nerve activity responses to physiological stressors in humans. Hypertension 44, 732-738.

Moreira LB, Fuchs SC, Wiehe M, Gus M, Moraes RS \& Fuchs FD. (2008). Incidence of hypertension in Porto Alegre, Brazil: a population-based study. Journal of Human Hypertension 22, 48-50.

Mori TA. (2006). Omega-3 fatty acids and hypertension in humans. Clinical and Experimental Pharmacology and Physiology 33, 842-846.

Mori TA, Watts GF, Burke V, Hilme E, Puddey IB \& Beilin LJ. (2000). Differential effects of eicosapentaenoic acid and docosahexaenoic acid on vascular reactivity of the forearm microcirculation in hyperlipidemic, overweight men. Circulation 102, 1264-1269.

Mori TA \& Woodman RJ. (2006). The independent effects of eicosapentaenoic acid and docosahexaenoic acid on cardiovascular risk factors in humans. Current Opinion in Clinical Nutrition and Metabolic Care 9, 95-104.

Morris MC, Sacks F \& Rosner B. (1993). Does fish oil lower blood pressure? A metaanalysis of controlled trials. Circulation 88, 523-533.

Mozaffarian D, Geelen A, Brouwer IA, Geleijnse JM, Zock PL \& Katan MB. (2005). Effect of fish oil on heart rate in humans: a meta-analysis of randomized controlled trials.

Circulation 112, 1945-1952.

Mozaffarian D, Stein PK, Prineas RJ \& Siscovick DS. (2008). Dietary fish and omega-3 fatty acid consumption and heart rate variability in US adults. Circulation 117, 11301137.

Neal B, MacMahon S \& Chapman N. (2000). Effects of ACE inhibitors, calcium antagonists, and other blood-pressure-lowering drugs: results of prospectively designed overviews of randomised trials. Blood Pressure Lowering Treatment Trialists' Collaboration. Lancet 356, 1955-1964.

Newton HF, Zwemer, R.L., Cannon, W.B. (1930). Studies on the Conditions of Activity in Endocrine Organs: The Mystery of Emotional Acceleration of the Denervated Heart after Exclusion of Known Humoral Accelerators. American Journal of Physiology 96, 377-391. 
$\mathrm{Ng} \mathrm{AV}$, Callister R, Johnson DG \& Seals DR. (1994). Sympathetic neural reactivity to stress does not increase with age in healthy humans. American Journal of Physiology 267, H344-353.

Nishimura M, Nanbu A, Komori T, Ohtsuka K, Takahashi H \& Yoshimura M. (2000). Eicosapentaenoic acid stimulates nitric oxide production and decreases cardiac noradrenaline in diabetic rats. Clinical and Experimental Pharmacology and Physiology 27, 618-624.

Noll G, Tschudi M, Nava E \& Luscher TF. (1997). Endothelium and high blood pressure. International Journal of Microcirculation: Clinical and Experimental 17, 273-279.

Noll G, Wenzel RR, Schneider M, Oesch V, Binggeli C, Shaw S, Weidmann P \& Luscher TF. (1996). Increased activation of sympathetic nervous system and endothelin by mental stress in normotensive offspring of hypertensive parents. Circulation 93, 866-869.

Norris GW. (1917). Blood-pressure. Lea \& Febiger, Philadelphia and New York,. Pagani M, Lombardi F, Guzzetti S, Rimoldi O, Furlan R, Pizzinelli P, Sandrone G, Malfatto G, Dell'Orto S, Piccaluga E \& et al. (1986). Power spectral analysis of heart rate and arterial pressure variabilities as a marker of sympatho-vagal interaction in man and conscious dog. Circulation Research 59, 178-193.

Parati G, Di Rienzo M, Bertinieri G, Pomidossi G, Casadei R, Groppelli A, Pedotti A, Zanchetti A \& Mancia G. (1988). Evaluation of the baroreceptor-heart rate reflex by 24hour intra-arterial blood pressure monitoring in humans. Hypertension 12, 214-222.

Pomeranz B, Macaulay RJ, Caudill MA, Kutz I, Adam D, Gordon D, Kilborn KM, Barger AC, Shannon DC, Cohen RJ \& et al. (1985). Assessment of autonomic function in humans by heart rate spectral analysis. American Journal of Physiology 248, H151-153.

Radomski MW, Palmer RM \& Moncada S. (1987). Comparative pharmacology of endothelium-derived relaxing factor, nitric oxide and prostacyclin in platelets. British Journal of Pharmacology 92, 181-187.

Reims HM, Fossum E, Høieggen A, Moan A, Eide I \& Kjeldsen SE. (2004). Adrenal medullary overactivity in lean, borderline hypertensive young men. American Journal of Hypertension 17, 611-618.

Richards AM, Nicholls MG, Espiner EA, Ikram H, Cullens M \& Hinton D. (1986). Diurnal patterns of blood pressure, heart rate and vasoactive hormones in normal man. Clinical and Experimental Hypertension. Part A, Theory and Practice 8, 153-166.

Roddie IC. (1977). Human responses to emotional stress. Irish Journal of Medical Science 146, 395-417.

Roger VL, Go AS, Lloyd-Jones DM, Adams RJ, Berry JD, Brown TM, Carnethon MR, Dai S, de Simone G, Ford ES, Fox CS, Fullerton HJ, Gillespie C, Greenlund KJ, Hailpern SM, Heit JA, Ho PM, Howard VJ, Kissela BM, Kittner SJ, Lackland DT, Lichtman JH, 
Lisabeth LD, Makuc DM, Marcus GM, Marelli A, Matchar DB, McDermott MM, Meigs JB, Moy CS, Mozaffarian D, Mussolino ME, Nichol G, Paynter NP, Rosamond WD, Sorlie PD, Stafford RS, Turan TN, Turner MB, Wong ND \& Wylie-Rosett J. (2011). Heart disease and stroke statistics--2011 update: a report from the American Heart Association. Circulation 123, e18-e209.

Rousseau-Ralliard D, Moreau D, Guilland JC, Raederstorff D \& Grynberg A. (2009). Docosahexaenoic acid, but not eicosapentaenoic acid, lowers ambulatory blood pressure and shortens interval QT in spontaneously hypertensive rats in vivo. Prostaglandins Leukotrienes and Essential Fatty Acids 80, 269-277.

Ruediger H, Seibt R, Scheuch K, Krause M \& Alam S. (2004). Sympathetic and parasympathetic activation in heart rate variability in male hypertensive patients under mental stress. Journal of Human Hypertension 18, 307-315.

Rusch NJ, Shepherd JT, Webb RC \& Vanhoutte PM. (1981). Different behavior of the resistance vessels of the human calf and forearm during contralateral isometric exercise, mental stress, and abnormal respiratory movements. Circulation Research 48, I118-130.

Rushmer RF, Smith O \& Franklin D. (1959). Mechanisms of cardiac control in exercise. Circulation Research 7, 602-627.

Santangelo K, Falkner B \& Kushner H. (1989). Forearm hemodynamics at rest and stress in borderline hypertensive adolescents. American Journal of Hypertension 2, 5256 .

Selye H. (1936). A Syndrome produced by diverse nocuous agents. Nature 138, 32.

Selye H \& Fortier C. (1950). Adaptive reaction to stress. Psychosomatic Medicine 12, 149-157.

Shepherd JT. (1987). Circulatory response to exercise in health. Circulation 76, VI3-10.

Shepherd JT \& Katusic ZS. (1991). Endothelium-derived vasoactive factors: I. Endothelium-dependent relaxation. Hypertension 18, III76-85.

Simopoulos AP. (2008). The importance of the omega-6/omega-3 fatty acid ratio in cardiovascular disease and other chronic diseases. Experimental Biology and Medicine (Maywood) 233, 674-688.

Singer P. (1990). Blood pressure-lowering effect of mackerel diet. Klinische Wochenschrift 68 Suppl 20, 40-48.

Spieker LE, Hurlimann D, Ruschitzka F, Corti R, Enseleit F, Shaw S, Hayoz D, Deanfield JE, Luscher TF \& Noll G. (2002). Mental stress induces prolonged endothelial dysfunction via endothelin-A receptors. Circulation 105, 2817-2820. 
Steinback CD, Salmanpour A, Breskovic T, Dujic Z \& Shoemaker JK. (2010). Sympathetic neural activation: an ordered affair. Journal of Physiology (London) 588, 4825-4836.

Sundlof G \& Wallin BG. (1977). The variability of muscle nerve sympathetic activity in resting recumbent man. Journal of Physiology (London) 272, 383-397.

Sundlof G \& Wallin BG. (1978). Human muscle nerve sympathetic activity at rest. Relationship to blood pressure and age. Journal of Physiology (London) 274, 621-637.

Takeshita A, Imaizumi T, Ashihara T, Yamamoto K, Hoka S \& Nakamura M. (1982). Limited maximal vasodilator capacity of forearm resistance vessels in normotensive young men with a familial predisposition to hypertension. Circulation Research 50, 671677.

Terano T, Salmon JA, Higgs GA \& Moncada S. (1986). Eicosapentaenoic acid as a modulator of inflammation. Effect on prostaglandin and leukotriene synthesis.

Biochemical Pharmacology 35, 779-785.

Thies F, Garry JM, Yaqoob P, Rerkasem K, Williams J, Shearman CP, Gallagher PJ, Calder PC \& Grimble RF. (2003). Association of $n-3$ polyunsaturated fatty acids with stability of atherosclerotic plaques: a randomised controlled trial. Lancet 361, 477-485.

Tidgren B \& Hjemdahl P. (1989). Renal responses to mental stress and epinephrine in humans. American Journal of Physiology 257, F682-689.

Timio M, Verdecchia P, Venanzi S, Gentili S, Ronconi M, Francucci B, Montanari M \& Bichisao E. (1988). Age and blood pressure changes. A 20 -year follow-up study in nuns in a secluded order. Hypertension 12, 457-461.

Vallbo AB \& Hagbarth KE. (1967). Impulses recorded with micro-electrodes in human muscle nerves during stimulation of mechanoreceptors and voluntary contractions. Electroencephalography and Clinical Neurophysiology 23, 392.

Vallbo AB, Hagbarth KE \& Wallin BG. (2004). Microneurography: how the technique developed and its role in the investigation of the sympathetic nervous system. Journal of Applied Physiology 96, 1262-1269.

van den Hoogen PC, Feskens EJ, Nagelkerke NJ, Menotti A, Nissinen A \& Kromhout D. (2000). The relation between blood pressure and mortality due to coronary heart disease among men in different parts of the world. Seven Countries Study Research Group. New England Journal of Medicine 342, 1-8.

Vasan RS, Larson MG, Leip EP, Evans JC, O'Donnell CJ, Kannel WB \& Levy D. (2001). Impact of high-normal blood pressure on the risk of cardiovascular disease. New England Journal of Medicine 345, 1291-1297. 
Wallin BG. (1984). Muscle sympathetic activity and plasma concentrations of noradrenaline. Acta Physiologica Scandinavica. Supplementum 527, 21-24.

Wallin BG, Delius W \& Hagbarth KE. (1973). Comparison of sympathetic nerve activity in normotensive and hypertensive subjects. Circulation Research 33, 9-21.

Wallin BG, Esler M, Dorward P, Eisenhofer G, Ferrier C, Westerman R \& Jennings G. (1992). Simultaneous measurements of cardiac noradrenaline spillover and sympathetic outflow to skeletal muscle in humans. Journal of Physiology (London) 453, 45-58.

Wallin BG, Sundlöf G, Eriksson BM, Dominiak P, Grobecker H \& Lindblad LE. (1981). Plasma noradrenaline correlates to sympathetic muscle nerve activity in normotensive man. Acta Physiologica Scandinavica 111, 69-73.

Westenberg HG \& Liebowitz MR. (2004). Overview of panic and social anxiety disorders. Journal of Clinical Psychiatry 65 Suppl 14, 22-26.

Widgren BR, Wikstrand J, Berglund G \& Andersson OK. (1992). Increased response to physical and mental stress in men with hypertensive parents. Hypertension 20, 606-611.

Wilkinson DJ, Thompson JM, Lambert GW, Jennings GL, Schwarz RG, Jefferys D, Turner AG \& Esler MD. (1998). Sympathetic activity in patients with panic disorder at rest, under laboratory mental stress, and during panic attacks. Archives of General Psychiatry 55, 511-520.

Wilson TD, Cotter LA, Draper JA, Misra SP, Rice CD, Cass SP \& Yates BJ. (2006). Vestibular inputs elicit patterned changes in limb blood flow in conscious cats. Journal of Physiology (London) 575, 671-684.

Yamagishi K, Iso H, Date C, Fukui M, Wakai K, Kikuchi S, Inaba Y, Tanabe N \& Tamakoshi A. (2008). Fish, omega-3 polyunsaturated fatty acids, and mortality from cardiovascular diseases in a nationwide community-based cohort of Japanese men and women the JACC (Japan Collaborative Cohort Study for Evaluation of Cancer Risk) Study. Journal of the American College of Cardiology 52, 988-996.

Zhang Y, Lee ET, Devereux RB, Yeh J, Best LG, Fabsitz RR \& Howard BV. (2006). Prehypertension, diabetes, and cardiovascular disease risk in a population-based sample: the Strong Heart Study. Hypertension 47, 410-414. 


\section{Appendix A}

Table A.1. Raw data for subject characteristics in normotensive group for Study 1.

\begin{tabular}{cccccc} 
Subject & Age (yrs) & Height $(\mathbf{c m})$ & Weight $\mathbf{( k g )}$ & BMI $\left(\mathbf{k g} / \mathbf{m}^{2}\right)$ & Gender \\
\hline 1 & 25 & 176 & 100 & 32 & Male \\
2 & 18 & 173 & 71 & 24 & Male \\
3 & 19 & 177 & 60 & 19 & Male \\
4 & 23 & 179 & 66 & 21 & Male \\
5 & 20 & 167 & 63 & 23 & Male \\
6 & 18 & 185 & 98 & 29 & Male \\
7 & 39 & 168 & 60 & 21 & Male \\
8 & 19 & 196 & 106 & 28 & Male \\
9 & 20 & 185 & 60 & 18 & Male \\
10 & 19 & 177 & 75 & 24 & Male \\
11 & 22 & 184 & 80 & 24 & Male \\
12 & 18 & 170 & 63 & 22 & Male \\
13 & 20 & 174 & 79 & 26 & Male \\
14 & 23 & 161 & 70 & 27 & Male \\
15 & 22 & 181 & 72 & 22 & Male \\
16 & 19 & 179 & 91 & 28 & Male \\
17 & 40 & 184 & 89 & 26 & Male \\
18 & 30 & 170 & 77 & 27 & Male
\end{tabular}

BMI; Body Mass Index 
Table A.2. Raw data for subject characteristics in prehypertensive group for Study 1.

\begin{tabular}{cccccc} 
Subject & Age (yrs) & Height $(\mathbf{c m})$ & Weight $\mathbf{( k g )}$ & BMI $\left(\mathbf{k g} / \mathbf{m}^{\mathbf{2}}\right)$ & Gender \\
\hline 1 & 32 & 184 & 97 & 29 & Male \\
2 & 19 & 178 & 83 & 26 & Male \\
3 & 20 & 178 & 84 & 27 & Male \\
4 & 22 & 178 & 84 & 27 & Male \\
5 & 21 & 176 & 77 & 25 & Male \\
6 & 21 & 179 & 87 & 27 & Male \\
7 & 23 & 183 & 92 & 27 & Male \\
8 & 21 & 179 & 67 & 21 & Male \\
9 & 19 & 185 & 81 & 24 & Male \\
10 & 18 & 166 & 85 & 31 & Male \\
11 & 20 & 189 & 92 & 26 & Male \\
12 & 20 & 174 & 95 & 31 & Male \\
13 & 23 & 177 & 76 & 24 & Male \\
14 & 21 & 184 & 84 & 25 & Male \\
15 & 21 & 173 & 82 & 27 & Male \\
16 & 28 & 189 & 86 & 24 & Male \\
17 & 20 & 174 & 81 & 27 & Male
\end{tabular}

BMI; Body Mass Index 
Table A.3 Raw data for seated resting measurements in normotensive group for Study 1.

$\begin{array}{ccccc}\text { Subject } & \begin{array}{c}\text { SAP } \\ \text { (mmHg) }\end{array} & \begin{array}{c}\text { DAP } \\ \text { (mmHg) }\end{array} & \begin{array}{c}\text { MAP } \\ \text { (mmHg) }\end{array} & \begin{array}{c}\text { HR } \\ \text { (beats/min) }\end{array} \\ 1 & 116.3 & 61.6 & 79.8 & 71.0 \\ 2 & 116.3 & 62.1 & 80.2 & 74.8 \\ 3 & 107.8 & 64.2 & 78.7 & 82.8 \\ 4 & 110.0 & 57.0 & 74.7 & 60.0 \\ 5 & 108.6 & 68.2 & 81.7 & 73.6 \\ 6 & 116.4 & 76.2 & 89.6 & 68.9 \\ 7 & 103.3 & 65.0 & 77.8 & 68.9 \\ 8 & 117.4 & 69.6 & 85.5 & 63.9 \\ 9 & 109.0 & 56.0 & 73.7 & 58.1 \\ 10 & 109.3 & 63.4 & 78.7 & 47.7 \\ 11 & 113.6 & 65.6 & 81.6 & 72.3 \\ 12 & 111.7 & 65.2 & 80.7 & 68.0 \\ 13 & 103.4 & 66.2 & 78.6 & 60.3 \\ 14 & 115.7 & 62.9 & 80.5 & 78.9 \\ 15 & 106.0 & 51.6 & 69.7 & 73.3 \\ 16 & 119.0 & 65.0 & 83.0 & 59.4 \\ 17 & 111.0 & 67.2 & 81.8 & 77.9 \\ 18 & 116.2 & 71.4 & 86.3 & 73.3\end{array}$

SAP; Systolic Arterial Pressure

DAP; Diastolic Arterial Pressure

MAP; Mean Arterial Pressure

HR; Heart Rate 
Table A.4. Raw data for seated resting measurements in prehypertensive group for Study 1

$\begin{array}{ccccc}\text { Subject } & \begin{array}{c}\text { SAP } \\ (\mathbf{m m H g})\end{array} & \begin{array}{c}\text { DAP } \\ (\mathbf{m m H g})\end{array} & \begin{array}{c}\text { MAP } \\ (\mathbf{m m H g})\end{array} & \begin{array}{c}\text { HR } \\ \text { (beats/min) }\end{array} \\ 1 & 120.3 & 66.4 & 84.4 & 60.6 \\ 2 & 132.9 & 77.0 & 95.6 & 69.6 \\ 3 & 118.0 & 81.0 & 93.3 & 81.8 \\ 4 & 125.0 & 71.0 & 89.0 & 84.0 \\ 5 & 139.9 & 77.4 & 98.2 & 71.4 \\ 6 & 139.7 & 84.4 & 102.8 & 59.1 \\ 7 & 133.0 & 66.0 & 88.3 & 77.0 \\ 8 & 124.7 & 69.1 & 87.6 & 80.7 \\ 9 & 121.5 & 67.5 & 85.5 & 68.6 \\ 10 & 122.4 & 72.8 & 89.3 & 95.0 \\ 11 & 122.0 & 63.6 & 83.1 & 64.3 \\ 12 & 121.2 & 78.8 & 92.9 & 95.0 \\ 13 & 133.4 & 73.8 & 93.7 & 72.3 \\ 14 & 123.6 & 69.6 & 87.6 & 88.9 \\ 15 & 121.9 & 50.2 & 74.1 & 55.7 \\ 16 & 128.6 & 69.0 & 88.9 & 75.7 \\ 17 & 125.6 & 62.4 & 83.5 & 71.1\end{array}$

SAP; Systolic Arterial Pressure

DAP; Diastolic Arterial Pressure

MAP; Mean Arterial Pressure

HR; Heart Rate 
Table A.5. Raw data for 5 minute average measurements of systolic arterial pressure as measured by the finometer in normotensive group for Study 1.

\begin{tabular}{cccc} 
Subject & Base & MS & Rec \\
\hline 1 & 138.5 & 158.3 & 154.3 \\
2 & 133.9 & 145.4 & 134.9 \\
3 & 115.5 & 114.2 & 104.1 \\
4 & 108.4 & 132.6 & 110.6 \\
5 & 127.7 & 135.2 & 130.9 \\
6 & 124.0 & 134.0 & 117.1 \\
7 & 123.1 & 124.0 & 112.7 \\
8 & 121.7 & 124.0 & 120.8 \\
9 & 122.1 & 133.3 & 131.0 \\
10 & 120.6 & 118.8 & 124.9 \\
11 & 128.5 & 144.7 & 135.3 \\
12 & 127.1 & 124.3 & 129.7 \\
13 & 118.6 & 121.5 & 118.6 \\
14 & 133.8 & 147.7 & 137.9 \\
15 & 116.0 & 131.7 & 108.0 \\
16 & 129.1 & 133.1 & 132.9 \\
17 & 129.6 & 137.2 & 127.8 \\
18 & 120.6 & 139.1 & 132.3
\end{tabular}

Base; Baseline

MS; Mental Stress

Rec; Recovery 
Table A.6. Raw data for 5 minute average measurements of systolic arterial pressure as measured by the finometer in prehypertensive group for Study 1 .

\begin{tabular}{cccc} 
Subject & Base & MS & Rec \\
\hline 1 & 124.8 & 135.7 & 129.3 \\
2 & 141.2 & 150.1 & 146.9 \\
3 & 138.6 & 145.0 & 133.2 \\
4 & 107.3 & 130.8 & 122.7 \\
5 & 141.5 & 157.1 & 145.4 \\
$6^{*}$ & -- & -- & -- \\
7 & 122.1 & 152.1 & 131.4 \\
8 & 129.7 & 155.6 & 135.1 \\
9 & 123.7 & 134.8 & 131.0 \\
10 & 121.7 & 123.7 & 121.7 \\
11 & 117.7 & 129.4 & 114.5 \\
12 & 110.1 & 118.2 & 112.4 \\
13 & 136.4 & 146.4 & 141.7 \\
14 & 118.8 & 138.9 & 123.8 \\
15 & 123.6 & 137.3 & 129.1 \\
16 & 129.2 & 144.2 & 132.8 \\
17 & 144.2 & 155.9 & 141.7
\end{tabular}

* Subject data not collected due to Finometer equipment malfunction during protocol.

Base; Baseline

MS; Mental Stress

Rec; Recovery 
Table A.7. Raw data for 5 minute average measurements of diastolic arterial pressure as measured by the finometer in normotensive group for Study 1 .

\begin{tabular}{cccc} 
Subject & Base & MS & Rec \\
\hline 1 & 78.3 & 94.4 & 87.0 \\
2 & 76.7 & 88.1 & 76.5 \\
3 & 79.8 & 85.2 & 76.1 \\
4 & 60.7 & 71.9 & 59.9 \\
5 & 67.4 & 76.2 & 68.5 \\
6 & 72.1 & 76.2 & 69.9 \\
7 & 66.8 & 74.2 & 65.2 \\
8 & 71.9 & 75.0 & 74.1 \\
9 & 71.1 & 80.5 & 76.2 \\
10 & 71.6 & 74.9 & 75.0 \\
11 & 68.7 & 79.6 & 72.3 \\
12 & 73.4 & 78.9 & 74.5 \\
13 & 73.5 & 79.1 & 75.0 \\
14 & 83.2 & 95.4 & 86.3 \\
15 & 68.5 & 80.9 & 67.4 \\
16 & 68.8 & 72.4 & 70.3 \\
17 & 71.5 & 77.8 & 71.6 \\
18 & 72.8 & 82.4 & 76.7
\end{tabular}


Table A.8. Raw data 5 minute average measurements of diastolic arterial pressure as measured by the finometer in prehypertensive group for Study 1.

\begin{tabular}{cccc} 
Subject & Base & MS & Rec \\
\hline 1 & 74.7 & 81.7 & 75.9 \\
2 & 74.1 & 83.6 & 73.8 \\
3 & 81.9 & 92.9 & 82.4 \\
4 & 80.8 & 92.1 & 78.5 \\
5 & 75.6 & 87.5 & 77.6 \\
$6^{*}$ & -- & -- & -- \\
7 & 71.4 & 94.3 & 73.2 \\
8 & 70.9 & 87.0 & 72.7 \\
9 & 71.9 & 83.1 & 73.2 \\
10 & 70.0 & 73.6 & 70.4 \\
11 & 64.4 & 72.4 & 65.9 \\
12 & 66.8 & 76.4 & 67.3 \\
13 & 84.5 & 89.2 & 88.4 \\
14 & 71.8 & 82.3 & 73.7 \\
15 & 66.3 & 79.4 & 69.9 \\
16 & 70.2 & 80.8 & 72.7 \\
17 & 68.4 & 79.4 & 69.4
\end{tabular}

* Subject data not collected due to Finometer equipment malfunction during protocol.

Base; Baseline

MS; Mental Stress

Rec; Recovery 
Table A.9. Raw data 5 minute average measurements of mean arterial pressure as measured by the finometer in normotensive group for Study 1.

\begin{tabular}{cccc} 
Subject & Base & MS & Rec \\
\hline 1 & 97.2 & 116.6 & 109.5 \\
2 & 98.5 & 110.7 & 98.6 \\
3 & 93.5 & 97.5 & 88.2 \\
4 & 76.3 & 93.1 & 77.7 \\
5 & 88.6 & 98.9 & 91.2 \\
6 & 89.2 & 94.6 & 86.3 \\
7 & 87.5 & 92.6 & 83.7 \\
8 & 87.3 & 90.9 & 89.2 \\
9 & 87.7 & 99.1 & 94.9 \\
10 & 85.8 & 90.2 & 90.9 \\
11 & 89.1 & 103.6 & 94.3 \\
12 & 91.1 & 95.7 & 93.5 \\
13 & 89.1 & 95.0 & 91.1 \\
14 & 101.2 & 116.2 & 105.7 \\
15 & 82.2 & 99.4 & 80.6 \\
16 & 88.2 & 93.3 & 90.9 \\
17 & 90.9 & 98.9 & 91.7 \\
18 & 90.4 & 104.5 & 97.6
\end{tabular}

Base; Baseline

MS; Mental Stress

Rec; Recovery 
Appendix A.10. Raw data for 5 minute average measurements of mean arterial pressure as measured by the finometer in prehypertensive group for Study 1 .

\begin{tabular}{cccc} 
Subject & Base & MS & Rec \\
\hline 1 & 90.4 & 101.0 & 93.1 \\
2 & 97.9 & 110.3 & 100.3 \\
3 & 100.6 & 111.9 & 100.6 \\
4 & 90.7 & 107.6 & 93.9 \\
5 & 97.5 & 115.4 & 101.1 \\
$6^{*}$ & -- & -- & -- \\
7 & 90.0 & 114.0 & 93.8 \\
8 & 90.1 & 111.3 & 93.2 \\
9 & 90.1 & 102.4 & 93.4 \\
10 & 83.8 & 89.5 & 85.1 \\
11 & 79.5 & 90.4 & 81.3 \\
12 & 80.8 & 91.6 & 82.3 \\
13 & 102.3 & 111.9 & 107.5 \\
14 & 85.5 & 101.4 & 89.2 \\
15 & 84.0 & 99.4 & 88.9 \\
16 & 89.7 & 103.5 & 93.5 \\
17 & 88.6 & 101.9 & 88.7
\end{tabular}

* Subject data not collected due to Finometer equipment malfunction during protocol.

Base; Baseline

MS; Mental Stress

Rec; Recovery 
Table A.11. Raw data for 5 minute average measurements of heart rate as measured by the finometer in normotensive group for Study 1.

\begin{tabular}{cccc} 
Subject & Base & MS & Rec \\
\hline 1 & 67.7 & 94.9 & 75.5 \\
2 & 74.6 & 126.2 & 79.7 \\
3 & 65.7 & 98.1 & 65.1 \\
4 & 51.1 & 73.8 & 50.4 \\
5 & 64.1 & 80.6 & 63.3 \\
6 & 68.8 & 73.5 & 69.5 \\
7 & 61.6 & 76.4 & 59.2 \\
8 & 57.2 & 60.1 & 57.5 \\
9 & 48.8 & 74.8 & 49.5 \\
10 & 47.7 & 72.9 & 48.6 \\
11 & 66.7 & 91.8 & 67.5 \\
12 & 62.0 & 92.6 & 61.7 \\
13 & 52.9 & 69.9 & 53.5 \\
14 & 58.0 & 74.3 & 57.8 \\
15 & 58.2 & 75.7 & 57.4 \\
16 & 54.6 & 72.8 & 58.6 \\
17 & 60.8 & 68.0 & 61.6 \\
18 & 64.0 & 88.1 & 67.0
\end{tabular}

Base; Baseline

MS; Mental Stress

Rec; Recovery 
Table A.12 Raw data for 5 minute average measurements of heart rate as measured by the finometer in prehypertensive group for Study 1.

\begin{tabular}{cccc} 
Subject & Base & MS & Rec \\
\hline 1 & 51.2 & 64.8 & 53.4 \\
2 & 71.0 & 87.9 & 67.7 \\
3 & 72.0 & 83.7 & 76.0 \\
4 & 81.2 & 88.3 & 77.1 \\
5 & 67.4 & 84.3 & 65.0 \\
6 & 49.6 & 73.6 & 54.0 \\
7 & 71.2 & 128.9 & 80.9 \\
8 & 80.3 & 114.2 & 83.2 \\
9 & 62.0 & 73.8 & 64.6 \\
10 & 71.9 & 92.7 & 78.8 \\
11 & 52.9 & 70.6 & 58.0 \\
12 & 77.4 & 88.9 & 77.4 \\
13 & 69.1 & 86.6 & 70.0 \\
14 & 79.1 & 86.2 & 78.6 \\
15 & 51.8 & 66.4 & 54.5 \\
16 & 63.5 & 72.1 & 65.8 \\
17 & 63.5 & 76.1 & 60.9
\end{tabular}

Base; Baseline

MS; Mental Stress

Rec; Recovery 
Table A.13. Raw data for 5 minute average measurements of muscle sympathetic nerve activity (MSNA) bursts/min in normotensive group for Study 1.

\begin{tabular}{cccc} 
Subject & Base & MS & Rec \\
\hline 1 & 8.6 & 12.4 & 6.4 \\
2 & 2.6 & -- & -- \\
3 & -- & -- & -- \\
4 & 6.6 & 12.6 & 11.6 \\
5 & 6.2 & 24.2 & 7.8 \\
6 & 15.6 & 21.2 & 19.2 \\
7 & 9.2 & 21.0 & 16.4 \\
8 & 6.0 & -- & -- \\
9 & 4.0 & 5.8 & 3.2 \\
10 & -- & -- & -- \\
11 & -- & -- & -- \\
12 & 8.8 & 11.8 & 13.2 \\
13 & 16.0 & 17.0 & 28.0 \\
14 & 10.6 & -- & -- \\
15 & 4.0 & 14.0 & 9.2 \\
16 & 10.4 & -- & -- \\
17 & 29.4 & 37.2 & 29.6 \\
18 & 8.2 & -- & --
\end{tabular}

Missing data is due to either loss of nerve recordings during protocol, or unsuccessful location of nerve activity.

Base; Baseline

MS; Mental Stress

Rec; Recovery 
Table A.14. Raw data for 5 minute average measurements of MSNA bursts/min in prehypertensive group for Study 1 .

\begin{tabular}{cccc} 
Subject & Base & MS & Rec \\
\hline 1 & 7.0 & -- & -- \\
2 & 27.0 & 31.7 & -- \\
3 & 25.2 & 29.2 & 28.8 \\
4 & 11.4 & 32.4 & 17.0 \\
5 & -- & -- & -- \\
6 & 3.4 & -- & -- \\
7 & 8.2 & 19.0 & 23.4 \\
8 & 3.8 & 9.6 & 6.2 \\
9 & 2.8 & 6.2 & 6.0 \\
10 & 7.2 & -- & -- \\
11 & 18.0 & -- & -- \\
12 & 7.0 & -- & -- \\
13 & 10.8 & 12.6 & 15.2 \\
14 & 17.0 & 11.8 & 17.8 \\
15 & 8.0 & 13.6 & 13.0 \\
16 & 4.5 & 9.0 & 7.3 \\
17 & 6.4 & 20.3 & --
\end{tabular}

Missing data is due to either loss of nerve recordings during protocol, or unsuccessful location of nerve activity.

Base; Baseline

MS; Mental Stress

Rec; Recovery 
Table A.15. Raw data for 5 minute average measurements of MSNA bursts per 100 heart beats in normotensive group for study 1.

\begin{tabular}{cccc} 
Subject & Base & MS & Rec \\
\hline 1 & 12.7 & 13.2 & 8.4 \\
2 & 3.5 & -- & -- \\
3 & -- & -- & -- \\
4 & 12.9 & 17.9 & 23.1 \\
5 & 9.7 & 30.2 & 12.4 \\
6 & 23.1 & 29.1 & 28.3 \\
7 & 15.0 & 27.7 & 28.0 \\
8 & 10.9 & -- & -- \\
9 & 8.3 & 7.8 & 6.5 \\
10 & -- & -- & -- \\
11 & -- & -- & -- \\
12 & 14.2 & 12.8 & 21.5 \\
13 & 30.4 & 24.4 & 52.8 \\
14 & 18.4 & -- & - \\
15 & 6.96 & 18.6 & 16.1 \\
16 & 19.1 & -- & -- \\
17 & 48.6 & 54.6 & 48.2 \\
18 & 12.9 & -- & --
\end{tabular}

Missing data is due to either loss of nerve recordings during protocol, or unsuccessful location of nerve activity.

Base; Baseline

MS; Mental Stress

Rec; Recovery 
Table A.16. Raw data for 5 minute average measurements of MSNA bursts per 100 heart beats in prehypertensive group for study 1 .

\begin{tabular}{cccc} 
Subject & Base & MS & Rec \\
\hline 1 & 13.7 & -- & -- \\
2 & 38.7 & 36.8 & -- \\
3 & 35.0 & 35.1 & 38.0 \\
4 & 14.1 & 36.9 & 22.0 \\
5 & -- & -- & -- \\
6 & 7.0 & -- & -- \\
7 & 11.7 & 14.2 & 29.1 \\
8 & 4.8 & 8.4 & 7.4 \\
9 & 4.6 & 8.4 & 9.4 \\
10 & 10.5 & -- & -- \\
11 & 34.2 & -- & -- \\
12 & 9.1 & -- & -- \\
13 & 15.7 & 14.5 & 21.8 \\
14 & 21.7 & 13.8 & 22.7 \\
15 & 15.4 & 20.6 & 23.8 \\
16 & 7.2 & 12.5 & 11.2 \\
17 & 10.1 & 26.1 & --
\end{tabular}

Missing data is due to either loss of nerve recordings during protocol, or unsuccessful location of nerve activity.

Base; Baseline

MS; Mental Stress

Rec; Recovery 
Table A.17. Raw data for 5 minute average measurements of total MSNA (arbitrary units) in normotensive group for study 1 .

\begin{tabular}{cccc} 
Subject & Base & MS & Rec \\
\hline 1 & 4665.4 & 12323.9 & 4687.6 \\
2 & 2265.5 & -- & -- \\
3 & -- & -- & -- \\
4 & 3346.8 & 12917.3 & 9870.0 \\
5 & 3143.7 & 16129.0 & 2970.2 \\
6 & 7878.0 & 8252.9 & 6569.8 \\
7 & 2350.2 & 7873.3 & 3258.2 \\
8 & 2185.5 & -- & -- \\
9 & 1921.1 & 4301.4 & 1921.2 \\
10 & -- & -- & -- \\
11 & -- & -- & -- \\
12 & 4209.9 & 6218.5 & 4504.8 \\
13 & 6464.1 & 13705.9 & 11897.5 \\
14 & 6906.8 & -- & -- \\
15 & 1286.6 & 8854.8 & 3526.3 \\
16 & 4874.6 & -- & -- \\
17 & -- & -- & -- \\
18 & 3072.2 & -- & -
\end{tabular}

Missing data is due to either loss of nerve recordings during protocol, or unsuccessful location of nerve activity.

Base; Baseline

MS; Mental Stress

Rec; Recovery 
Table A.18. Raw data for 5 minute average measurements of total MSNA (arbitrary units) in prehypertensive group for study 1 .

\begin{tabular}{cccc} 
Subject & Base & MS & Rec \\
\hline 1 & 3919.4 & -- & -- \\
2 & 15174.3 & 25679.3 & -- \\
3 & 9522.4 & 26003.6 & 9210.4 \\
4 & 4552.6 & 22021.3 & 7797.7 \\
5 & -- & -- & -- \\
6 & 2048.1 & -- & -- \\
7 & -- & -- & -- \\
8 & 2427.1 & 7951.7 & 2560.0 \\
9 & 1349.4 & 4434.6 & 4417.1 \\
10 & 2875.2 & -- & -- \\
11 & 9547.2 & -- & -- \\
12 & 3173.9 & -- & -- \\
13 & 4089.3 & 14199.4 & 6910.5 \\
14 & 8623.5 & 9246.8 & 9207.2 \\
15 & 4086.3 & 7066.5 & 5199.3 \\
16 & 1722.9 & 3605.4 & 2304.8 \\
17 & 4365.1 & 13956.6 & --
\end{tabular}

Missing data is due to either loss of nerve recordings during protocol, or unsuccessful location of nerve activity.

Base; Baseline

MS; Mental Stress

Rec; Recovery 
Table A.19. Raw data for 5 minute average measurements of forearm blood flow $(\mathrm{mL} / 100 \mathrm{~mL} / \mathrm{min})$ in normotensive group for study 1 .

\begin{tabular}{cccc} 
Subject & Base & MS & Rec \\
\hline 1 & 2.78 & 4.01 & 2.87 \\
2 & 2.41 & 2.19 & 2.06 \\
3 & 2.02 & 2.74 & 2.04 \\
4 & -- & -- & -- \\
5 & 3.21 & 5.34 & 3.27 \\
6 & 2.63 & 3.14 & 2.75 \\
7 & 2.20 & 3.78 & 2.41 \\
8 & 3.88 & 5.80 & 3.93 \\
9 & 1.84 & 2.28 & 2.28 \\
10 & 3.04 & 3.57 & 3.37 \\
11 & 1.64 & 2.08 & 1.65 \\
12 & -- & -- & -- \\
13 & 1.74 & 2.91 & 1.51 \\
14 & 2.05 & 2.61 & 1.90 \\
15 & 1.59 & 2.60 & 1.84 \\
16 & 1.01 & 1.42 & 1.00 \\
17 & 3.60 & 3.05 & 3.11 \\
18 & 2.92 & 6.23 & 2.90
\end{tabular}

Missing data is due to either artifact during protocol, or mechanical issues with the equipment.

Base; Baseline

MS; Mental Stress

Rec; Recovery 
Table A.20. Raw data for 5 minute average measurements of forearm blood flow $(\mathrm{mL} / 100 \mathrm{~mL} / \mathrm{min})$ in prehypertensive group for study 1 .

\begin{tabular}{cccc} 
Subject & Base & MS & Rec \\
\hline 1 & 2.49 & 4.68 & 2.53 \\
2 & 2.69 & 3.54 & 2.47 \\
3 & 2.25 & 3.24 & 2.65 \\
4 & -- & -- & -- \\
5 & 4.21 & 6.91 & 3.96 \\
6 & 1.28 & 3.09 & 1.29 \\
7 & 5.34 & 10.77 & 6.17 \\
8 & 3.72 & 8.37 & 3.66 \\
9 & 2.65 & 3.87 & 3.38 \\
10 & 2.40 & 5.15 & 2.88 \\
11 & 3.61 & 6.15 & 3.54 \\
12 & 4.38 & 7.50 & 4.00 \\
13 & 3.43 & 4.36 & 3.90 \\
14 & 5.93 & 5.64 & 5.79 \\
15 & 3.68 & 4.88 & 3.92 \\
16 & 2.91 & 3.36 & 2.48 \\
17 & 4.15 & 5.22 & 3.19
\end{tabular}

Missing data is due to either artifact during protocol, or mechanical issues with the equipment.

Base; Baseline

MS; Mental Stress

Rec; Recovery 
Table A.21. Raw data for 5 minute average measurements of forearm vascular resistance $(\mathrm{mmHg} / \mathrm{mL} / 100 \mathrm{~mL} / \mathrm{min})$ in normotensive group for study 1 .

\begin{tabular}{cccc} 
Subject & Base & MS & Rec \\
\hline 1 & 16.99 & 12.19 & 16.32 \\
2 & 24.21 & 11.11 & 24.96 \\
3 & 33.66 & 12.66 & 28.28 \\
4 & 37.69 & 13.29 & 39.22 \\
5 & 44.76 & 20.22 & 50.66 \\
6 & 40.18 & 25.39 & 31.57 \\
7 & 33.84 & 28.18 & 37.46 \\
8 & -- & -- & -- \\
9 & 48.97 & 25.98 & 56.82 \\
10 & 49.85 & 28.39 & 51.41 \\
11 & 46.85 & 31.43 & 62.82 \\
12 & 41.18 & 16.75 & 49.94 \\
13 & 55.22 & 27.33 & 40.40 \\
14 & 41.67 & 29.03 & 44.41 \\
15 & 47.10 & 26.13 & 53.49 \\
16 & 48.08 & 30.23 & 44.59 \\
17 & 29.26 & 31.63 & 28.62 \\
18 & 30.98 & 18.01 & 33.73
\end{tabular}

Missing data is due to either artifact during protocol, or mechanical issues with the equipment.

Base; Baseline

MS; Mental Stress

Rec; Recovery 
Table A.22. Raw data for 5 minute average measurements of forearm vascular resistance $(\mathrm{mmHg} / \mathrm{mL} / 100 \mathrm{~mL} / \mathrm{min})$ in prehypertensive group for study 1 .

\begin{tabular}{cccc} 
Subject & Base & MS & Rec \\
\hline 1 & 36.57 & 24.07 & 37.17 \\
2 & 37.92 & 34.36 & 43.98 \\
3 & 44.86 & 36.57 & 38.45 \\
4 & -- & -- & -- \\
5 & 23.34 & 18.45 & 25.87 \\
6 & -- & -- & -- \\
7 & 16.87 & 10.67 & 15.34 \\
8 & 24.49 & 15.15 & 26.01 \\
9 & 34.72 & 27.66 & 30.63 \\
10 & 35.11 & 17.80 & 29.72 \\
11 & 23.21 & 17.57 & 23.02 \\
12 & 18.51 & 12.92 & 20.60 \\
13 & 29.98 & 26.22 & 28.01 \\
14 & 14.47 & 18.20 & 15.44 \\
15 & 23.14 & 20.44 & 22.96 \\
16 & 31.28 & 36.07 & 37.94 \\
17 & 21.38 & 20.81 & 27.85
\end{tabular}

Missing data is due to either artifact during protocol, or mechanical issues with the equipment.

Base; Baseline

MS; Mental Stress

Rec; Recovery 
Table A.23. Raw data for 5 minute average measurements of forearm vascular conductance $(\mathrm{mL} / 100 \mathrm{~mL} / \mathrm{min} / \mathrm{mmHg})$ in normotensive group for study 1 .

\begin{tabular}{cccc} 
Subject & Base & MS & Rec \\
\hline 1 & 0.060 & 0.084 & 0.062 \\
2 & 0.042 & 0.096 & 0.041 \\
3 & 0.030 & 0.084 & 0.037 \\
4 & 0.027 & 0.078 & 0.026 \\
5 & 0.022 & 0.064 & 0.020 \\
6 & 0.025 & 0.041 & 0.032 \\
7 & 0.030 & 0.036 & 0.027 \\
8 & -- & -- & -- \\
9 & 0.020 & 0.042 & 0.018 \\
10 & 0.020 & 0.039 & 0.020 \\
11 & 0.022 & 0.035 & 0.016 \\
12 & 0.025 & 0.063 & 0.021 \\
13 & 0.018 & 0.037 & 0.026 \\
14 & 0.024 & 0.035 & 0.023 \\
15 & 0.021 & 0.040 & 0.019 \\
16 & 0.021 & 0.034 & 0.022 \\
17 & 0.034 & 0.033 & 0.036 \\
18 & 0.032 & 0.060 & 0.030
\end{tabular}

Missing data is due to either artifact during protocol, or mechanical issues with the equipment.

Base; Baseline

MS; Mental Stress

Rec; Recovery 
Table A.24. Raw data for 5 minute average measurements of forearm vascular conductance $(\mathrm{mL} / 100 \mathrm{~mL} / \mathrm{min} / \mathrm{mmHg})$ in prehypertensive group for study 1 .

\begin{tabular}{cccc} 
Subject & Base & MS & Rec \\
\hline 1 & 0.028 & 0.047 & 0.027 \\
2 & 0.028 & 0.032 & 0.025 \\
3 & 0.022 & 0.029 & 0.026 \\
4 & -- & -- & -- \\
5 & 0.043 & 0.061 & 0.039 \\
6 & -- & -- & -- \\
7 & 0.059 & 0.094 & 0.066 \\
8 & 0.041 & 0.076 & 0.039 \\
9 & 0.029 & 0.037 & 0.036 \\
10 & 0.029 & 0.057 & 0.034 \\
11 & 0.045 & 0.069 & 0.044 \\
12 & 0.054 & 0.082 & 0.049 \\
13 & 0.034 & 0.039 & 0.036 \\
14 & 0.069 & 0.055 & 0.065 \\
15 & 0.044 & 0.049 & 0.044 \\
16 & 0.032 & 0.033 & 0.027 \\
17 & 0.047 & 0.052 & 0.036
\end{tabular}

Missing data is due to either artifact during protocol, or mechanical issues with the equipment.

Base; Baseline

MS; Mental Stress

Rec; Recovery 
Table A.25. Raw data for 5 minute average measurements of calf blood flow ( $\mathrm{mL} / 100 \mathrm{~mL} / \mathrm{min})$ in normotensive group for study 1 .

\begin{tabular}{cccc} 
Subject & Base & MS & Rec \\
\hline 1 & 3.18 & 4.34 & -- \\
2 & 3.58 & 4.50 & 3.50 \\
3 & 2.18 & 4.71 & 2.00 \\
4 & 1.21 & 1.20 & 1.12 \\
5 & 1.79 & 1.98 & 2.12 \\
6 & 1.94 & 2.28 & 2.07 \\
7 & 1.52 & 1.32 & 1.58 \\
8 & 2.51 & 3.17 & 2.27 \\
9 & 1.66 & 3.83 & 1.73 \\
10 & 1.84 & 3.10 & 1.92 \\
11 & 1.40 & 2.44 & 1.23 \\
12 & -- & -- & -- \\
13 & 3.02 & 3.46 & 2.26 \\
14 & 1.80 & 2.41 & 1.76 \\
15 & 2.38 & 2.77 & 1.42 \\
16 & 1.86 & 2.36 & 1.66 \\
17 & 1.60 & 1.72 & 1.56 \\
18 & 1.70 & 4.11 & 2.32
\end{tabular}

Missing data is due to either artifact during protocol, or mechanical issues with the equipment.

Base; Baseline

MS; Mental Stress

Rec; Recovery 
Table A.26. Raw data for 5 minute average measurements of calf blood flow ( $\mathrm{mL} / 100 \mathrm{~mL} / \mathrm{min}$ ) in prehypertensive group for study 1 .

\begin{tabular}{cccc} 
Subject & Base & MS & Rec \\
\hline 1 & 2.78 & 4.01 & 2.87 \\
2 & 2.41 & 2.19 & 2.06 \\
3 & 2.02 & 2.74 & 2.04 \\
4 & -- & -- & -- \\
5 & 3.21 & 5.34 & 3.27 \\
6 & 2.63 & 3.14 & 2.75 \\
7 & 2.20 & 3.78 & 2.41 \\
8 & 3.88 & 5.80 & 3.93 \\
9 & 1.84 & 2.28 & 2.28 \\
10 & 3.04 & 3.57 & 3.37 \\
11 & 1.64 & 2.08 & 1.65 \\
12 & -- & -- & -- \\
13 & 1.74 & 2.91 & 1.51 \\
14 & 2.05 & 2.61 & 1.90 \\
15 & 1.59 & 2.60 & 1.84 \\
16 & 1.01 & 1.42 & 1.00 \\
17 & 3.60 & 3.50 & 3.11
\end{tabular}

Missing data is due to either artifact during protocol, or mechanical issues with the equipment.

Base; Baseline

MS; Mental Stress

Rec; Recovery 
Table A.27. Raw data for 5 minute average measurements of calf vascular resistance $(\mathrm{mmHg} / \mathrm{mL} / 100 \mathrm{~mL} / \mathrm{min})$ in normotensive group for study 1 .

\begin{tabular}{cccc} 
Subject & Base & MS & Rec \\
\hline 1 & 31.03 & 32.37 & -- \\
2 & 27.66 & 26.09 & 30.37 \\
3 & 43.80 & 26.03 & 44.69 \\
4 & 63.18 & 82.52 & 70.01 \\
5 & 49.94 & 51.17 & 43.25 \\
6 & 46.00 & 43.00 & 41.78 \\
7 & 58.77 & 72.71 & 56.56 \\
8 & 35.00 & 29.06 & 40.28 \\
9 & 53.14 & 30.72 & 55.10 \\
10 & 46.66 & 32.05 & 47.79 \\
11 & 65.85 & 43.86 & 78.13 \\
12 & -- & -- & -- \\
13 & 29.54 & 27.59 & 41.58 \\
14 & 56.41 & 48.90 & 61.41 \\
15 & 34.71 & 36.27 & 58.14 \\
16 & 47.48 & 39.88 & 55.91 \\
17 & 57.38 & 57.52 & 59.33 \\
18 & 54.10 & 28.00 & 42.52
\end{tabular}

Missing data is due to either artifact during protocol, or mechanical issues with the equipment.

Base; Baseline

MS; Mental Stress

Rec; Recovery 
Table A.28. Raw data for 5 minute average measurements of calf vascular resistance $(\mathrm{mmHg} / \mathrm{mL} / 100 \mathrm{~mL} / \mathrm{min})$ in prehypertensive group for study 1 .

\begin{tabular}{cccc} 
Subject & Base & MS & Rec \\
\hline 1 & 32.54 & 28.64 & 32.48 \\
2 & 41.44 & 52.62 & 49.10 \\
3 & 49.91 & 42.67 & 49.57 \\
4 & -- & -- & -- \\
5 & 30.77 & 21.98 & 31.12 \\
6 & -- & -- & -- \\
7 & 41.22 & 33.78 & 39.25 \\
8 & 23.27 & 21.87 & 23.85 \\
9 & 49.26 & 45.69 & 41.68 \\
10 & 27.68 & 25.86 & 25.40 \\
11 & 49.30 & 43.84 & 49.50 \\
12 & -- & -- & -- \\
13 & 58.92 & 46.26 & 71.28 \\
14 & 41.84 & 39.78 & 47.12 \\
15 & 53.54 & 40.38 & 49.55 \\
16 & 89.62 & 81.05 & 96.48 \\
17 & 24.72 & 31.31 & 28.62
\end{tabular}

Missing data is due to either artifact during protocol, or mechanical issues with the equipment.

Base; Baseline

MS; Mental Stress

Rec; Recovery 
Table A.29. Raw data for 5 minute average measurements of calf vascular conductance $(\mathrm{mL} / 100 \mathrm{~mL} / \mathrm{min} / \mathrm{mmHg})$ in normotensive group for study 1 .

\begin{tabular}{cccc} 
Subject & Base & MS & Rec \\
\hline 1 & 0.033 & 0.037 & -- \\
2 & 0.036 & 0.041 & 0.035 \\
3 & 0.023 & 0.048 & 0.023 \\
4 & 0.016 & 0.013 & 0.014 \\
5 & 0.020 & 0.020 & 0.023 \\
6 & 0.022 & 0.024 & 0.024 \\
7 & 0.017 & 0.014 & 0.019 \\
8 & 0.029 & 0.035 & 0.025 \\
9 & 0.019 & 0.039 & 0.018 \\
10 & 0.021 & 0.034 & 0.021 \\
11 & 0.016 & 0.024 & 0.013 \\
12 & -- & -- & -- \\
13 & 0.034 & 0.036 & 0.025 \\
14 & 0.018 & 0.021 & 0.017 \\
15 & 0.029 & 0.028 & 0.018 \\
16 & 0.021 & 0.025 & 0.018 \\
17 & 0.018 & 0.017 & 0.017 \\
18 & 0.019 & 0.040 & 0.024
\end{tabular}

Missing data is due to either artifact during protocol, or mechanical issues with the equipment.

Base; Baseline

MS; Mental Stress

Rec; Recovery 
Table A.30. Raw data for 5 minute average measurements of calf vascular conductance $(\mathrm{mL} / 100 \mathrm{~mL} / \mathrm{min} / \mathrm{mmHg})$ in prehypertensive group for study 1 .

\begin{tabular}{cccc} 
Subject & Base & MS & Rec \\
\hline 1 & 0.031 & 0.040 & 0.031 \\
2 & 0.025 & 0.020 & 0.021 \\
3 & 0.020 & 0.024 & 0.020 \\
4 & -- & -- & -- \\
5 & 0.033 & 0.046 & 0.032 \\
6 & -- & -- & -- \\
7 & 0.024 & 0.033 & 0.026 \\
8 & 0.043 & 0.053 & 0.042 \\
9 & 0.020 & 0.022 & 0.024 \\
10 & 0.036 & 0.040 & 0.040 \\
11 & 0.021 & 0.023 & 0.020 \\
12 & -- & -- & -- \\
13 & 0.017 & 0.026 & 0.014 \\
14 & 0.024 & 0.025 & 0.021 \\
15 & 0.019 & 0.026 & 0.021 \\
16 & 0.011 & 0.014 & 0.011 \\
17 & 0.041 & 0.035 & 0.035
\end{tabular}

Missing data is due to either artifact during protocol, or mechanical issues with the equipment.

Base; Baseline

MS; Mental Stress

Rec; Recovery 


\section{Appendix B}

Table B.1. Baseline comparisons between normotensive ( $\mathrm{nt}$ ) and prehypertensive (pht) groups for Study 1. Independent t-tests are presented as two-tailed significance.

\begin{tabular}{|c|c|c|c|c|c|c|}
\hline \multirow[b]{2}{*}{ Variable } & \multirow{2}{*}{$\begin{array}{c}\text { Mean } \\
\text { Diff. }\end{array}$} & \multirow{2}{*}{$\begin{array}{l}\text { Std. } \\
\text { Error }\end{array}$} & \multicolumn{2}{|c|}{$95 \%$ Conf. Interval } & \multirow[t]{2}{*}{ Sig. } & \multirow{2}{*}{$\underset{\mathrm{nt} / \mathrm{pht}}{\mathbf{N}}$} \\
\hline & & & Lower & Upper & & \\
\hline Age (yrs) & -1.29 & 1.82 & -5.00 & 2.41 & .482 & $18 / 17$ \\
\hline BMI $\left(\mathrm{kg} / \mathrm{m}^{2}\right)$ & 1.75 & 1.12 & -.523 & 4.03 & .126 & $18 / 17$ \\
\hline SAP (mmHg) & 14.97 & 1.98 & 10.94 & 18.99 & .000 & $18 / 17$ \\
\hline $\mathrm{DAP}(\mathrm{mmHg})$ & 6.23 & 2.36 & 1.44 & 11.03 & .012 & $18 / 17$ \\
\hline $\mathrm{MAP}(\mathrm{mmHg})$ & 9.14 & 1.92 & 5.23 & 13.04 & .000 & $18 / 17$ \\
\hline HR (beats/min) & 6.24 & 3.50 & -.869 & 13.36 & .083 & $18 / 17$ \\
\hline MSNA (bursts/min) & .735 & 2.55 & -4.48 & 5.95 & .775 & $15 / 16$ \\
\hline MSNA (bursts/100hb) & -.612 & 3.96 & -8.72 & 7.49 & .878 & $15 / 16$ \\
\hline FBF (mL/100mL/min) & .922 & .393 & .120 & 1.72 & .026 & $17 / 16$ \\
\hline $\mathrm{FVR}(\mathrm{mmHg} / \mathrm{mL} / 100 \mathrm{~mL} / \mathrm{min})$ & -4.91 & 4.46 & -14.00 & 4.18 & .279 & $17 / 16$ \\
\hline $\mathrm{FVC}(\mathrm{mL} / 100 \mathrm{~mL} / \mathrm{min} / \mathrm{mmHg})$ & .0074 & .0048 & -.0023 & .017 & .129 & $17 / 16$ \\
\hline CBF (mL/100mL/min) & .308 & .259 & -.222 & .837 & .245 & $17 / 15$ \\
\hline CVR (mmHg/mL/100mL/min) & -.565 & 5.00 & -10.80 & 9.67 & .990 & $17 / 15$ \\
\hline CVC $(\mathrm{mL} / 100 \mathrm{~mL} / \mathrm{min} / \mathrm{mmHg})$ & .001 & .003 & -.006 & .008 & .753 & $17 / 15$ \\
\hline
\end{tabular}

Table B.2. Repeated Measures ANOVA of changes in 5 minute average $\Delta$ SAP $(\mathrm{mmHg})$ in Study 1,2 condition vs. 2 groups (Normotensive/Prehypertensive), presented as one-tailed significance.

\begin{tabular}{lc}
\multicolumn{1}{c}{ Source } & Base - Mental Stress \\
\hline Condition & .000 \\
Condition x Group & .050
\end{tabular}

Table B.3. Post-hoc paired t-test of changes in $\Delta$ SAP $(\mathrm{mmHg})$ base to mental stress.

\begin{tabular}{|c|c|c|c|c|c|c|}
\hline \multirow{2}{*}{ Group } & \multirow{2}{*}{ Mean } & \multirow{2}{*}{ Std. Dev. } & \multicolumn{2}{|c|}{ 95\% Confidence Interval } & \multirow{2}{*}{ Sig. } & \multirow{2}{*}{$\mathbf{N}$} \\
\hline & & & Lower & Upper & & \\
\hline Norm & 8.90 & 8.07 & 4.89 & 12.92 & .000 & 18 \\
\hline Prehypertensive & 14.01 & 7.47 & 10.00 & 18.00 & .000 & 16 \\
\hline
\end{tabular}

Table B.4. Post-hoc independent t-test of changes in $\Delta$ SAP $(\mathrm{mmHg})$ base to mental stress.

\begin{tabular}{|c|c|c|c|c|c|}
\hline \multirow{2}{*}{ Group } & \multirow{2}{*}{$\begin{array}{l}\text { Mean } \\
\text { Diff. }\end{array}$} & \multirow{2}{*}{$\begin{array}{l}\text { Std. } \\
\text { Error }\end{array}$} & \multicolumn{2}{|c|}{ 95\% Confidence Interval } & \multirow{2}{*}{ Sig. } \\
\hline & & & Lower & Upper & \\
\hline $\begin{array}{l}\text { Normotensive vs. } \\
\text { Prehypertensive }\end{array}$ & 5.11 & 2.68 & -.344 & 10.57 & .033 \\
\hline
\end{tabular}


Table B.5. Repeated Measures ANOVA of changes in 5 minute average $\triangle \mathrm{DAP}(\mathrm{mmHg})$ in Study 1,2 condition vs. 2 groups (Normotensive/Prehypertensive), presented as one-tailed significance.

\begin{tabular}{lc}
\multicolumn{1}{c}{ Source } & Base - Mental Stress \\
\hline Condition & .000 \\
Condition x Group & .065
\end{tabular}

Table B.6. Post-hoc paired t-test of changes in $\triangle \mathrm{DAP}(\mathrm{mmHg})$ base to mental stress.

\begin{tabular}{ccccccc} 
Group & Mean & Std. Dev. & $\begin{array}{c}\text { 95\% Confidence Interval } \\
\text { Lower }\end{array}$ & Upper & Sig. & N \\
\hline Normotensive & 8.12 & 3.77 & 6.25 & 9.99 & .000 & 18 \\
Prehypertensive & 10.76 & 4.43 & 8.40 & 13.12 & .000 & 16
\end{tabular}

Table B.7. Post-hoc independent t-test of changes in $\triangle$ DAP $(\mathrm{mmHg})$ base to mental stress.

\begin{tabular}{|c|c|c|c|c|c|}
\hline \multirow{2}{*}{ Group } & \multirow{2}{*}{ Mean } & \multirow{2}{*}{$\begin{array}{l}\text { Std. } \\
\text { Error }\end{array}$} & \multicolumn{2}{|c|}{$95 \%$ Confidence Interval } & \multirow{2}{*}{ Sig. } \\
\hline & & & Lower & Upper & \\
\hline $\begin{array}{l}\text { Normotensive vs. } \\
\text { Prehypertensive }\end{array}$ & 2.64 & 1.41 & -.225 & 5.50 & .035 \\
\hline
\end{tabular}

Table B.8. Repeated Measures ANOVA of changes in 5 minute average $\triangle$ MAP ( $\mathrm{mmHg}$ ) in Study 1,2 condition vs. 2 groups (Normotensive/Prehypertensive), presented as one-tailed significance.

\begin{tabular}{lc}
\multicolumn{1}{c}{ Source } & Base - Mental Stress \\
\hline Condition & .000 \\
Condition x Group & .017
\end{tabular}

Table B.9. Post-hoc paired t-test of changes in $\triangle M A P(\mathrm{mmHg})$ base to mental stress.

\begin{tabular}{|c|c|c|c|c|c|c|}
\hline \multirow{2}{*}{ Group } & \multirow{2}{*}{ Mean } & \multirow{2}{*}{ Std. Dev. } & \multicolumn{2}{|c|}{ 95\% Confidence Interval } & \multirow{2}{*}{ Sig. } & \multirow{2}{*}{$\mathbf{N}$} \\
\hline & & & Lower & Upper & & \\
\hline Normotensive & 9.83 & 5.33 & 7.18 & 12.48 & .000 & 18 \\
\hline Prehypertensive & 13.90 & 4.56 & 11.46 & 16.33 & .000 & 16 \\
\hline
\end{tabular}


Table B.10. Post-hoc independent t-test of changes in $\triangle M A P(\mathrm{mmHg})$ base to mental stress.

\begin{tabular}{|c|c|c|c|c|c|}
\hline \multirow{2}{*}{ Group } & \multirow{2}{*}{ Mean } & \multirow{2}{*}{$\begin{array}{l}\text { Std. } \\
\text { Error }\end{array}$} & \multicolumn{2}{|c|}{$95 \%$ Confidence Interval } & \multirow{2}{*}{ Sig } \\
\hline & & & Lower & Upper & \\
\hline $\begin{array}{l}\text { Normotensive vs. } \\
\text { Prehypertensive }\end{array}$ & 4.07 & 1.71 & .577 & 7.56 & .017 \\
\hline
\end{tabular}

Table B.11. Repeated Measures ANOVA of changes in 5 minute average $\Delta \mathbf{H R}$ (beats $/ \mathrm{min}$ ) in Study 1, 2 condition vs. 2 groups (Normotensive/Prehypertensive), presented as one-tailed significance.

\begin{tabular}{lc}
\multicolumn{1}{c}{ Source } & Base - Mental Stress \\
\hline Condition & .000 \\
Condition x Group & .195
\end{tabular}

Table B.12. Post-hoc paired t-test of changes in $\Delta H R$ (beats $/ \mathrm{min}$ ) base to mental stress.

\begin{tabular}{|c|c|c|c|c|c|c|}
\hline \multirow{2}{*}{ Group } & \multirow{2}{*}{ Mean } & \multirow{2}{*}{ Std. Dev. } & \multicolumn{2}{|c|}{ 95\% Confidence Interval } & \multirow{2}{*}{ Sig. } & \multirow{2}{*}{$\mathbf{N}$} \\
\hline & & & Lower & Upper & & \\
\hline Norm & 21.12 & 11.30 & 15.50 & 26.74 & .000 & 18 \\
\hline Prehypertensive & 17.49 & 12.49 & 10.84 & 24.15 & .000 & 16 \\
\hline
\end{tabular}

Table B.13. Repeated Measures ANOVA of changes in 5 minute average $\triangle M S N A$ (bursts $/ \mathrm{min}$ ) in Study 1, 2 condition vs. 2 groups (Normotensive/Prehypertensive), presented as one-tailed significance.

\begin{tabular}{lc}
\multicolumn{1}{c}{ Source } & Base - Mental Stress \\
\hline Condition & .000 \\
Condition x Group & .479
\end{tabular}

Table B.14. Post-hoc paired t-test of changes in $\Delta$ MSNA (bursts/min) base to mental stress.

\begin{tabular}{|c|c|c|c|c|c|c|}
\hline \multirow{2}{*}{ Group } & \multirow{2}{*}{ Mean } & \multirow{2}{*}{ Std. Dev. } & \multicolumn{2}{|c|}{$95 \%$ Confidence Interval } & \multirow{2}{*}{ Sig. } & \multirow{2}{*}{$\mathbf{N}$} \\
\hline & & & Lower & Upper & & \\
\hline Normote & 6.88 & 5.23 & 3.14 & 10.62 & .001 & 10 \\
\hline Prehypertensive & 6.39 & 6.84 & 1.80 & 10.98 & .005 & 11 \\
\hline
\end{tabular}


Table B.15. Repeated Measures ANOVA of changes in 5 minute average $\triangle$ Total MSNA (arbitrary units) in Study 1, 2 condition vs. 2 groups (Normotensive/Prehypertensive), presented as one-tailed significance.

\begin{tabular}{lc}
\multicolumn{1}{c}{ Source } & Base - Mental Stress \\
\hline Condition & .000 \\
Condition x Group & .244
\end{tabular}

Table B.16. Post-hoc paired t-test of changes in $\Delta$ Total MSNA (arbitrary units) base to mental stress.

\begin{tabular}{ccccccc} 
Group & \multirow{2}{*}{ Mean } & Std. Dev. & 95\% Confidence Interval & Sig. & N \\
& & & Lower & Upper & S & \\
\hline Normotensive & 6145.7 & 4015.7 & 3058.9 & 9232.4 & .001 & 9 \\
Prehypertensive & 7825.2 & 5980.2 & 3547.3 & 12103.2 & .002 & 10
\end{tabular}

Table B.17. Repeated Measures ANOVA of changes in 5 minute average $\Delta F B F(\%)$ in Study 1,2 condition vs. 2 groups (Normotensive/Prehypertensive), presented as one-tailed significance.

\begin{tabular}{lc}
\multicolumn{1}{c}{ Source } & Base - Mental Stress \\
\hline Condition & .000 \\
Condition x Group & .005
\end{tabular}

Table B.18. Post-hoc paired t-test of changes in $\triangle F B F(\%)$ base to mental stress.

\begin{tabular}{|c|c|c|c|c|c|c|}
\hline \multirow{2}{*}{ Group } & \multirow{2}{*}{ Mean } & \multirow{2}{*}{ Std. Dev. } & \multicolumn{2}{|c|}{ 95\% Confidence Interval } & \multirow{2}{*}{ Sig. } & \multirow{2}{*}{$\mathbf{N}$} \\
\hline & & & Lower & Upper & & \\
\hline Norn & 116.03 & 65.68 & 82.26 & 149.8 & .000 & 17 \\
\hline Prehypertensive & 62.18 & 42.55 & 39.51 & 84.85 & .000 & 16 \\
\hline
\end{tabular}

Table B.19. Post-hoc independent t-test of changes in $\Delta F B F(\%)$ base to mental stress.

\begin{tabular}{|c|c|c|c|c|c|}
\hline \multirow{2}{*}{ Group } & \multirow{2}{*}{ Mean } & \multirow{2}{*}{$\begin{array}{l}\text { Std. } \\
\text { Error }\end{array}$} & \multicolumn{2}{|c|}{ 95\% Confidence Interval } & \multirow{2}{*}{ Sig. } \\
\hline & & & Lower & Upper & \\
\hline $\begin{array}{l}\text { Normotensive vs. } \\
\text { Prehypertensive }\end{array}$ & 53.84 & 19.15 & 14.58 & 93.11 & .005 \\
\hline
\end{tabular}


Table B.20. Repeated Measures ANOVA of changes in 5 minute average $\Delta$ FVR (\%) in Study 1 , 2 condition vs. 2 groups (Normotensive/Prehypertensive), presented as one-tailed significance.

\begin{tabular}{lc}
\multicolumn{1}{c}{ Source } & Base - Mental Stress \\
\hline Condition & .000 \\
Condition x Group & .001
\end{tabular}

Table B.21. Post-hoc paired t-test of changes in $\Delta F V R(\%)$ base to mental stress.

\begin{tabular}{|c|c|c|c|c|c|c|}
\hline \multirow{2}{*}{ Group } & \multirow{2}{*}{ Mean } & \multirow{2}{*}{ Std. Dev. } & \multicolumn{2}{|c|}{ 95\% Confidence Interval } & \multirow{2}{*}{ Sig. } & \multirow{2}{*}{$\mathbf{N}$} \\
\hline & & & Lower & Upper & & \\
\hline Norm & -40.97 & 18.10 & -50.27 & -31.66 & .000 & 17 \\
\hline Prehypertensive & -17.85 & 20.02 & -28.93 & -6.77 & .002 & 15 \\
\hline
\end{tabular}

Table B.22. Post-hoc independent t-test of changes in $\Delta F V R(\%)$ base to mental stress.

\begin{tabular}{|c|c|c|c|c|c|}
\hline \multirow{2}{*}{ Group } & \multirow{2}{*}{ Mean } & \multirow{2}{*}{$\begin{array}{l}\text { Std. } \\
\text { Error }\end{array}$} & \multicolumn{2}{|c|}{ 95\% Confidence Interval } & \multirow{2}{*}{ Sig } \\
\hline & & & Lower & Upper & \\
\hline $\begin{array}{l}\text { Normotensive vs. } \\
\text { Prehypertensive }\end{array}$ & -23.12 & 6.73 & -36.87 & -9.36 & .001 \\
\hline
\end{tabular}

Table B.23. Repeated Measures ANOVA of changes in 5 minute average $\Delta \mathbf{F V C}(\%)$ in Study 1 , 2 condition vs. 2 groups (Normotensive/Prehypertensive), presented as one-tailed significance.

\begin{tabular}{lc}
\multicolumn{1}{c}{ Source } & Base - Mental Stress \\
\hline Condition & .000 \\
Condition x Group & .001
\end{tabular}

Table B.24. Post-hoc paired t-test of changes in $\Delta F V C(\%)$ base to mental stress.

\begin{tabular}{|c|c|c|c|c|c|c|}
\hline \multirow{2}{*}{ Group } & \multirow{2}{*}{ Mean } & \multirow{2}{*}{ Std. Dev. } & \multicolumn{2}{|c|}{$95 \%$ Confidence Interval } & \multirow{2}{*}{ Sig. } & \multirow{2}{*}{$\mathbf{N}$} \\
\hline & & & Lower & Upper & & \\
\hline Normc & 94.47 & 57.57 & 64.87 & 124.07 & .000 & 17 \\
\hline Prehypertensive & 36.53 & 32.60 & 18.48 & 54.59 & .000 & 15 \\
\hline
\end{tabular}


Table B.25. Post-hoc independent t-test of changes in $\Delta \mathrm{FVC}(\%)$ base to mental stress.

\begin{tabular}{|c|c|c|c|c|c|}
\hline \multirow{2}{*}{ Group } & \multirow{2}{*}{ Mean } & \multirow{2}{*}{$\begin{array}{l}\text { Std. } \\
\text { Error }\end{array}$} & \multicolumn{2}{|c|}{ 95\% Confidence Interval } & \multirow{2}{*}{ Sig. } \\
\hline & & & Lower & Upper & \\
\hline $\begin{array}{l}\text { Normotensive vs. } \\
\text { Prehypertensive }\end{array}$ & 57.93 & 16.30 & 24.41 & 91.46 & .000 \\
\hline
\end{tabular}

Table B.26. Repeated Measures ANOVA of changes in 5 minute average $\Delta$ CBF (\%) in Study 1 , 2 condition vs. 2 groups (Normotensive/Prehypertensive), presented as one-tailed significance.

\begin{tabular}{lc}
\multicolumn{1}{c}{ Source } & Base - Mental Stress \\
\hline Condition & .000 \\
Condition x Group & .302
\end{tabular}

Table B.27. Post-hoc paired t-test of changes in $\triangle \mathrm{CBF}(\%)$ base to mental stress.

\begin{tabular}{|c|c|c|c|c|c|c|}
\hline \multirow{2}{*}{ Group } & \multirow{2}{*}{ Mean } & \multirow{2}{*}{ Std. Dev. } & \multicolumn{2}{|c|}{$95 \%$ Confidence Interval } & \multirow{2}{*}{ Sig. } & \multirow{2}{*}{$\mathbf{N}$} \\
\hline & & & Lower & Upper & & \\
\hline Normote & 43.14 & 46.58 & 19.19 & 67.09 & .001 & 17 \\
\hline Prehypertensive & 36.08 & 24.92 & 22.28 & 49.88 & .000 & 15 \\
\hline
\end{tabular}

Table B.28. Repeated Measures ANOVA of changes in 5 minute average $\mathbf{\Delta C V R}(\%)$ in Study 1 , 2 condition vs. 2 groups (Normotensive/Prehypertensive), presented as one-tailed significance.

\begin{tabular}{lc}
\multicolumn{1}{c}{ Source } & Base - Mental Stress \\
\hline Condition & .005 \\
Condition x Group & .313
\end{tabular}

Table B.29. Post-hoc paired t-test of changes in $\Delta$ CVR (\%) base to mental stress.

\begin{tabular}{|c|c|c|c|c|c|c|}
\hline \multirow{2}{*}{ Group } & \multirow{2}{*}{ Mean } & \multirow{2}{*}{ Std. Dev. } & \multicolumn{2}{|c|}{ 95\% Confidence Interval } & \multirow{2}{*}{ Sig. } & \multirow{2}{*}{$\mathbf{N}$} \\
\hline & & & Lower & Upper & & \\
\hline Norm & -11.47 & 22.36 & -22.96 & .029 & .025 & 17 \\
\hline Prehypertensive & -7.93 & 16.39 & -17.39 & 1.54 & .047 & 14 \\
\hline
\end{tabular}


Table B.30. Repeated Measures ANOVA of changes in 5 minute average $\mathbf{\Delta C V C}(\%)$ in Study 1 , 2 condition vs. 2 groups (Normotensive/Prehypertensive), presented as one-tailed significance.

\begin{tabular}{lc}
\multicolumn{1}{c}{ Source } & Base - Mental Stress \\
\hline Condition & .000 \\
Condition x Group & .210
\end{tabular}

Table B.31. Post-hoc paired t-test of changes in $\triangle \mathrm{CVC}(\%)$ base to mental stress.

\begin{tabular}{|c|c|c|c|c|c|c|}
\hline \multirow{2}{*}{ Group } & \multirow{2}{*}{ Mean } & \multirow{2}{*}{ Std. Dev. } & \multicolumn{2}{|c|}{ 95\% Confidence Interval } & \multirow{2}{*}{ Sig. } & \multirow{2}{*}{$\mathbf{N}$} \\
\hline & & & Lower & Upper & & \\
\hline Norm & 29.03 & 42.24 & 7.31 & 50.75 & .006 & 17 \\
\hline Prehypertensive & 18.90 & 20.25 & 7.21 & 30.59 & .002 & 14 \\
\hline
\end{tabular}




\section{Appendix C}

Table C.1. Anthropometric data for the normotensive group in study 2

\begin{tabular}{|c|c|c|c|c|c|c|c|c|c|c|}
\hline \multirow[b]{2}{*}{$\begin{array}{l}\text { Sub- } \\
\text { ject }\end{array}$} & \multirow[b]{2}{*}{$\begin{array}{c}\text { Gend } \\
\text { er }\end{array}$} & \multirow[b]{2}{*}{ Drug } & \multicolumn{4}{|c|}{ Pre Supplementation } & \multicolumn{4}{|c|}{ Post Supplementation } \\
\hline & & & $\begin{array}{l}\text { Age } \\
\text { yr }\end{array}$ & $\begin{array}{l}\text { Height } \\
\mathrm{cm}\end{array}$ & $\begin{array}{l}\text { Weight } \\
\text { kg }\end{array}$ & $\begin{array}{c}\mathrm{BMI} \\
\mathrm{kg} / \mathrm{m}^{2}\end{array}$ & $\begin{array}{c}\text { Age } \\
\mathrm{yr}\end{array}$ & $\begin{array}{c}\text { Height } \\
\mathrm{cm}\end{array}$ & $\begin{array}{l}\text { Weight } \\
\text { kg }\end{array}$ & $\begin{array}{c}\mathrm{BMI} \\
\mathrm{kg} / \mathrm{m}^{2}\end{array}$ \\
\hline 1 & $M$ & FO & 25 & 176 & 99.5 & 32 & 25 & 176 & 100.5 & 33 \\
\hline 2 & $\mathrm{~F}$ & PL & 39 & 176 & 79.5 & 26 & 39 & 175 & 79.5 & 26 \\
\hline 3 & $M$ & FO & 18 & 173 & 71.0 & 24 & 19 & 173 & 72.0 & 24 \\
\hline 4 & $\mathrm{~F}$ & FO & 18 & 166 & 61.0 & 22 & 18 & 166 & 62.0 & 22 \\
\hline 5 & $\mathrm{M}$ & FO & 19 & 177 & 60.0 & 19 & 19 & 177 & 60.5 & 19 \\
\hline 6 & $\mathrm{~F}$ & PL & 18 & 161 & 68.0 & 26 & 18 & 162 & 69.0 & 26 \\
\hline 7 & $\mathrm{~F}$ & PL & 18 & 163 & 56.0 & 21 & 18 & 163 & 57.0 & 21 \\
\hline 8 & $\mathrm{~F}$ & PL & 19 & 174 & 68.0 & 22 & 19 & 174 & 69.5 & 23 \\
\hline 9 & $\mathrm{~F}$ & FO & 20 & 169 & 69.5 & 24 & 20 & 169 & 72.0 & 25 \\
\hline 10 & $\mathrm{~F}$ & PL & 21 & 152 & 60.5 & 26 & 21 & 157 & 61.5 & 25 \\
\hline 11 & $M$ & FO & 23 & 179 & 66.0 & 21 & 23 & 181 & 66.0 & 20 \\
\hline 12 & $\mathrm{~F}$ & FO & 20 & 168 & 62.5 & 22 & 20 & 169 & 64.0 & 22 \\
\hline 13 & $\mathrm{~F}$ & FO & 21 & 160 & 57.0 & 22 & 21 & 163 & 57.5 & 22 \\
\hline 14 & $\mathrm{~F}$ & FO & 20 & 155 & 52.0 & 22 & 20 & 154 & 52.0 & 22 \\
\hline 15 & $\mathrm{~F}$ & FO & 23 & 171 & 55.0 & 19 & 23 & 171 & 56.5 & 19 \\
\hline 16 & $\mathrm{~F}$ & FO & 22 & 149 & 62.5 & 28 & 22 & 149 & 64.0 & 29 \\
\hline 17 & $\mathrm{~F}$ & PL & 18 & 161 & 65.5 & 25 & 18 & 161 & 65.5 & 25 \\
\hline 18 & $\mathrm{~F}$ & FO & 21 & 163 & 57.5 & 22 & 22 & 163 & 55.5 & 21 \\
\hline 19 & $\mathrm{~F}$ & PL & 21 & 166 & 74.0 & 27 & 21 & 166 & 73.0 & 26 \\
\hline 20 & $M$ & PL & 20 & 167 & 63.0 & 23 & 20 & 168 & 62.5 & 22 \\
\hline 21 & $M$ & PL & 18 & 185 & 97.5 & 28 & 19 & 185 & 101.5 & 30 \\
\hline 22 & $M$ & PL & 39 & 168 & 60.0 & 21 & 39 & 167 & 60.5 & 22 \\
\hline 23 & $M$ & PL & 20 & 185 & 60.0 & 18 & 20 & 185 & 60.0 & 18 \\
\hline 24 & $M$ & FO & 19 & 177 & 75.0 & 24 & 19 & 177 & 74.0 & 24 \\
\hline 25 & $\mathrm{~F}$ & PL & 20 & 163 & 57.0 & 21 & 20 & 163 & 56.0 & 21 \\
\hline 26 & $M$ & FO & 22 & 184 & 80.0 & 24 & 22 & 186 & 77.0 & 22 \\
\hline 27 & $M$ & FO & 18 & 170 & 62.5 & 22 & 19 & 168 & 63.0 & 22 \\
\hline 28 & $M$ & PL & 20 & 174 & 79.0 & 26 & 20 & 171 & 80.0 & 27 \\
\hline 29 & $M$ & PL & 23 & 161 & 69.8 & 27 & 23 & 171 & 71.0 & 24 \\
\hline 30 & $M$ & PL & 22 & 181 & 72.0 & 22 & 22 & 182 & 72.0 & 22 \\
\hline 31 & $\mathrm{~F}$ & PL & 21 & 173 & 72.0 & 24 & 21 & 171 & 72.0 & 25 \\
\hline 32 & $\mathrm{~F}$ & FO & 21 & 169 & 77.6 & 27 & 21 & 169 & 79.0 & 28 \\
\hline 33 & $M$ & FO & 40 & 184 & 89.0 & 26 & 40 & 186 & 87.0 & 25 \\
\hline 34 & $M$ & FO & 30 & 170 & 77.0 & 27 & 30 & 170 & 77.5 & 27 \\
\hline 35 & $\mathrm{~F}$ & PL & 52 & 178 & 77.5 & 24 & 52 & 179 & 78.0 & 24 \\
\hline 36 & $F$ & FO & 53 & 163 & 62.5 & 24 & 54 & 162 & 62.5 & 24 \\
\hline 37 & $M$ & PL & 19 & 170 & 76.0 & 26 & 19 & 170 & 74.5 & 26 \\
\hline 38 & $M$ & PL & 25 & 173 & 81.5 & 27 & 25 & 173 & 80.0 & 27 \\
\hline
\end{tabular}

M, Male; F, Female; FO, Fish Oil; PL, Placebo; BMI, Body Mass Index 
Table C.2. Anthropometric data for prehypertensive group in Study 2

\begin{tabular}{|c|c|c|c|c|c|c|c|c|c|c|}
\hline \multirow[b]{2}{*}{$\begin{array}{c}\text { Subj- } \\
\text { ect }\end{array}$} & \multirow[b]{2}{*}{$\begin{array}{l}\text { Gen- } \\
\text { der }\end{array}$} & \multirow[b]{2}{*}{ Drug } & \multicolumn{4}{|c|}{ Pre Supplementation } & \multicolumn{4}{|c|}{ Post Supplementation } \\
\hline & & & $\begin{array}{c}\text { Age } \\
\mathrm{vr}\end{array}$ & $\begin{array}{c}\text { Height } \\
\mathrm{cm}\end{array}$ & $\begin{array}{c}\text { Weight } \\
\mathrm{kq}\end{array}$ & $\begin{array}{c}\mathrm{BMI} \\
\mathrm{kg} / \mathrm{m}^{2}\end{array}$ & $\begin{array}{c}\text { Age } \\
\mathrm{yr}\end{array}$ & $\begin{array}{c}\text { Height } \\
\mathrm{cm}\end{array}$ & $\begin{array}{c}\text { Weight } \\
\mathrm{kq}\end{array}$ & $\begin{array}{c}\mathrm{BMI} \\
\mathrm{kg} / \mathrm{m}^{2}\end{array}$ \\
\hline 39 & $M$ & FO & 32 & 184 & 97.0 & 29 & 32 & 184 & 97.0 & 29 \\
\hline 40 & $M$ & PL & 19 & 178 & 82.5 & 26 & 19 & 179 & 82.5 & 26 \\
\hline 41 & $M$ & PL & 20 & 178 & 84.0 & 27 & 20 & 178 & 82.0 & 26 \\
\hline 42 & $M$ & $\mathrm{FO}$ & 21 & 176 & 77.0 & 25 & 21 & 175 & 79.0 & 26 \\
\hline 43 & $M$ & PL & 21 & 179 & 87.0 & 27 & 21 & 180 & 87.5 & 27 \\
\hline 44 & $M$ & PL & 23 & 183 & 91.5 & 27 & 23 & 183 & 92.5 & 28 \\
\hline 45 & $M$ & FO & 21 & 179 & 66.5 & 21 & 21 & 179 & 67.0 & 21 \\
\hline 46 & $M$ & $\mathrm{FO}$ & 19 & 185 & 81.0 & 24 & 19 & 184 & 85.0 & 25 \\
\hline 47 & $M$ & PL & 18 & 166 & 85.0 & 31 & 18 & 166 & 77.5 & 28 \\
\hline 48 & $M$ & PL & 20 & 189 & 92.0 & 26 & 21 & 189 & 91.0 & 25 \\
\hline 49 & $M$ & PL & 20 & 174 & 95.0 & 31 & 20 & 174 & 96.0 & 32 \\
\hline 50 & $M$ & FO & 23 & 177 & 75.5 & 24 & 23 & 172 & 75.0 & 25 \\
\hline 51 & $M$ & FO & 21 & 184 & 83.5 & 25 & 22 & 184 & 82.0 & 24 \\
\hline 52 & $M$ & FO & 20 & 174 & 80.5 & 27 & 20 & 174 & 82.0 & 27 \\
\hline 53 & $M$ & FO & 26 & 185 & 95.0 & 28 & 26 & 186 & 97.5 & 28 \\
\hline 54 & $M$ & FO & 20 & 185 & 113.0 & 33 & 20 & 184 & 112.5 & 33 \\
\hline 55 & $M$ & PL & 31 & 184 & 91.0 & 27 & 31 & 183 & 91.0 & 27 \\
\hline 56 & $M$ & PL & 52 & 192 & 102.5 & 28 & 52 & 192 & 101.5 & 28 \\
\hline 57 & $M$ & FO & 33 & 173 & 97.0 & 32 & 34 & 172 & 96.5 & 33 \\
\hline 58 & $M$ & FO & 19 & 176 & 87.0 & 28 & 20 & 176 & 84.0 & 27 \\
\hline 59 & $\mathrm{~F}$ & PL & 50 & 164 & 81.0 & 30 & 50 & 164 & 82.0 & 30 \\
\hline 60 & $M$ & PL & 18 & 176 & 90.5 & 29 & 18 & 175 & 92.0 & 30 \\
\hline 61 & $M$ & FO & 18 & 176 & 75.5 & 24 & 18 & 176 & 77.0 & 25 \\
\hline 62 & $M$ & PL & 20 & 188 & 79.5 & 22 & 21 & 183 & 88.0 & 26 \\
\hline 63 & $M$ & FO & 23 & 170 & 92.0 & 32 & 23 & 170 & 92.0 & 32 \\
\hline 64 & $M$ & FO & 22 & 170 & 103.5 & 36 & 22 & 171 & 102.5 & 35 \\
\hline 65 & $M$ & PL & 19 & 172 & 67.5 & 23 & 19 & 172 & 66.5 & 22 \\
\hline 66 & $M$ & FO & 21 & 189 & 109.5 & 31 & 21 & 189 & 110.0 & 31 \\
\hline 67 & $M$ & PL & 19 & 179 & 87.5 & 27 & 19 & 179 & 86.5 & 27 \\
\hline
\end{tabular}

M, Male; F, Female; FO, Fish Oil; PL, Placebo; BMI, Body Mass Index 
Table C.3. Raw data for seated resting blood pressure $(\mathrm{mmHg})$ and heart rate (beats $/ \mathrm{min})$ for normotensive group in study 2 .

\begin{tabular}{|c|c|c|c|c|c|c|c|c|}
\hline \multirow[b]{2}{*}{ Subject } & \multicolumn{4}{|c|}{ Pre supplementation } & \multicolumn{4}{|c|}{ Post supplementation } \\
\hline & SAP & DAP & MAP & $\mathrm{HR}$ & SAP & DAP & MAP & $\mathrm{HR}$ \\
\hline 1 & 116.3 & 61.6 & 79.8 & 71.0 & 114.8 & 63.0 & 80.3 & 78.9 \\
\hline 2 & 104.0 & 66.0 & 78.7 & 75.1 & 104.2 & 63.0 & 76.7 & 70.8 \\
\hline 3 & 116.3 & 62.1 & 80.2 & 74.8 & 115.1 & 62.0 & 79.7 & 64.1 \\
\hline 4 & 117.9 & 70.0 & 86.0 & 80.3 & 109.9 & 68.3 & 82.2 & 80.4 \\
\hline 5 & 107.8 & 64.2 & 78.7 & 82.8 & 110.8 & 74.1 & 86.3 & 89.6 \\
\hline 6 & 104.1 & 68.0 & 80.0 & 90.6 & 107.3 & 68.0 & 81.1 & 95.7 \\
\hline 7 & 102.7 & 66.2 & 78.4 & 79.4 & 98.8 & 66.3 & 77.1 & 76.8 \\
\hline 8 & 99.1 & 57.8 & 71.6 & 68.9 & 101.8 & 61.8 & 75.1 & 68.3 \\
\hline 9 & 105.0 & 64.0 & 77.7 & 66.0 & 106.1 & 67.9 & 80.6 & 65.6 \\
\hline 10 & 97.0 & 67.0 & 77.0 & 71.0 & 100.0 & 66.7 & 77.8 & 71.6 \\
\hline 11 & 110.0 & 57.0 & 74.7 & 60.0 & 107.0 & 62.7 & 77.5 & 64.6 \\
\hline 12 & 106.0 & 64.9 & 78.6 & 68.2 & 114.9 & 72.1 & 86.4 & 71.3 \\
\hline 13 & 92.2 & 59.1 & 70.1 & 62.1 & 96.2 & 62.9 & 74.0 & 58.2 \\
\hline 14 & 106.7 & 70.3 & 82.4 & 85.2 & 103.7 & 71.9 & 82.5 & 84.6 \\
\hline 15 & 110.9 & 68.3 & 82.5 & 73.2 & 107.4 & 64.9 & 79.1 & 75.6 \\
\hline 16 & 104.9 & 65.7 & 78.8 & 73.7 & 97.8 & 65.4 & 76.2 & 65.2 \\
\hline 17 & 105.1 & 68.3 & 80.6 & 85.1 & 101.7 & 62.0 & 75.2 & 72.8 \\
\hline 18 & 99.6 & 72.9 & 81.8 & 81.1 & 90.2 & 63.2 & 72.2 & 87.9 \\
\hline 19 & 105.2 & 66.2 & 79.2 & 69.2 & 100.4 & 61.0 & 74.1 & 60.9 \\
\hline 20 & 108.6 & 68.2 & 81.7 & 73.6 & 109.4 & 70.7 & 83.6 & 65.9 \\
\hline 21 & 116.4 & 76.2 & 89.6 & 68.9 & 122.6 & 80.9 & 94.8 & 85.1 \\
\hline 22 & 103.3 & 65.0 & 77.8 & 68.9 & 107.4 & 66.2 & 79.9 & 73.6 \\
\hline 23 & 109 & 56.0 & 73.7 & 58.1 & 110.6 & 61.7 & 78.0 & 67.8 \\
\hline 24 & 109.3 & 63.4 & 78.7 & 47.7 & 109.6 & 60.9 & 77.1 & 47.7 \\
\hline 25 & 95.9 & 64.3 & 74.8 & 76.6 & 90.7 & 61.7 & 71.4 & 76.6 \\
\hline 26 & 113.6 & 65.6 & 81.6 & 72.3 & 111.5 & 69.7 & 83.6 & 67.3 \\
\hline 27 & 111.7 & 65.2 & 80.7 & 68 & 104.8 & 64.9 & 78.2 & 72.8 \\
\hline 28 & 103.4 & 66.2 & 78.6 & 60.3 & 106.8 & 69.8 & 82.1 & 67.3 \\
\hline 29 & 115.7 & 62.9 & 80.5 & 78.9 & 121.2 & 65.8 & 84.3 & 79.0 \\
\hline 30 & 106 & 51.6 & 69.7 & 73.3 & 116.7 & 61.6 & 80.0 & 86.4 \\
\hline 31 & 105.2 & 63.3 & 77.3 & 75.7 & 106.0 & 62.6 & 77.1 & 71.0 \\
\hline 32 & 111.8 & 66.4 & 81.5 & 77.2 & 100.7 & 64.3 & 76.4 & 69.6 \\
\hline 33 & 111 & 67.2 & 81.8 & 77.9 & 108.4 & 60.8 & 76.7 & 75.6 \\
\hline 34 & 116.2 & 71.4 & 86.3 & 73.3 & 108.0 & 70.6 & 83.1 & 72.0 \\
\hline 35 & 113.2 & 75.8 & 88.3 & 64.2 & 100.2 & 65.2 & 76.9 & 63.3 \\
\hline 36 & 113.3 & 72.2 & 85.9 & 56.1 & 118.1 & 72.9 & 88.0 & 58.6 \\
\hline 37 & 114.3 & 60.4 & 78.4 & 76.6 & 110.7 & 72.7 & 85.4 & 91.1 \\
\hline 38 & 118.6 & 66 & 83.5 & 92.4 & 111.6 & 71.2 & 84.7 & 94.8 \\
\hline
\end{tabular}

SAP, systolic arterial pressure DAP, diastolic arterial pressure MAP, mean arterial pressure $\mathrm{HR}$, heart rate 
Table C.4. Raw data for seated resting blood pressure $(\mathrm{mmHg})$ and heart rate (beats/min) for prehypertensive group in study 2

\begin{tabular}{ccccc|cccc} 
& \multicolumn{3}{c|}{ Pre supplementation } & \multicolumn{3}{c}{ Post supplementation } \\
\cline { 2 - 10 } Subject & SAP & DAP & MAP & HR & SAP & DAP & MAP & HR \\
39 & 120.3 & 66.4 & 84.4 & 60.6 & 123.4 & 68.6 & 86.9 & 60.9 \\
40 & 132.9 & 77.0 & 95.6 & 69.6 & 127.2 & 68.4 & 88.0 & 54.9 \\
41 & 118.0 & 81.0 & 93.3 & 81.8 & 119.4 & 79.0 & 92.5 & 78.4 \\
42 & 139.9 & 77.4 & 98.2 & 71.4 & 125.3 & 69.7 & 88.2 & 64.0 \\
43 & 139.7 & 84.4 & 102.8 & 59.1 & 121.2 & 71.9 & 88.3 & 58.2 \\
44 & 133.0 & 66.0 & 88.3 & 77.0 & 125.2 & 63.9 & 84.3 & 80.3 \\
45 & 124.7 & 69.1 & 87.6 & 80.7 & 128.7 & 69.9 & 89.5 & 89.2 \\
46 & 121.5 & 67.5 & 85.5 & 68.6 & 123.2 & 68.9 & 87.0 & 69.9 \\
47 & 122.4 & 72.8 & 89.3 & 95.0 & 131.1 & 78.4 & 96.0 & 90.6 \\
48 & 122.0 & 63.6 & 83.1 & 64.3 & 118.4 & 64.6 & 82.5 & 62.0 \\
49 & 121.2 & 78.8 & 92.9 & 95.0 & 121.6 & 82.7 & 95.7 & 90.9 \\
50 & 133.4 & 73.8 & 93.7 & 72.3 & 125.1 & 73.9 & 91.0 & 66.7 \\
51 & 123.6 & 69.6 & 87.6 & 88.9 & 121.6 & 66.3 & 84.7 & 86.3 \\
52 & 125.6 & 62.4 & 83.5 & 71.1 & 124.3 & 63.0 & 83.4 & 70.6 \\
53 & 127.9 & 66.6 & 87.0 & 69.8 & 132.2 & 74.4 & 93.7 & 77.8 \\
54 & 128.3 & 66.7 & 87.2 & 70.0 & 128.4 & 67.9 & 88.1 & 77.3 \\
55 & 124.6 & 68.4 & 87.1 & 80.6 & 123.7 & 74.3 & 90.8 & 75.4 \\
56 & 121.9 & 72.0 & 88.6 & 69.1 & 124.9 & 76.4 & 92.6 & 72.9 \\
57 & 125.2 & 79.9 & 95.0 & 75.1 & 126.0 & 78.0 & 94.0 & 75.0 \\
58 & 129.3 & 66.2 & 87.2 & 68.8 & 129.4 & 67.9 & 88.4 & 71.3 \\
59 & 115.7 & 82.7 & 93.7 & 80.5 & 111.8 & 76.1 & 88.0 & 77.9 \\
60 & 124.3 & 63.1 & 83.5 & 63.7 & 114.0 & 58.0 & 76.7 & 51.9 \\
61 & 122.6 & 76.8 & 92.1 & 96.3 & 115.7 & 65.6 & 82.3 & 78.6 \\
62 & 131.2 & 70.7 & 90.9 & 68.7 & 132.2 & 72.7 & 92.5 & 77.2 \\
63 & 126.0 & 65.2 & 85.5 & 75.8 & 117.4 & 63.1 & 81.2 & 71.2 \\
64 & 134.1 & 70.7 & 91.8 & 68.5 & 135.2 & 69.4 & 91.3 & 69.0 \\
65 & 133.2 & 78.7 & 96.9 & 78.5 & 132.6 & 82.0 & 98.9 & 81.1 \\
66 & 123.1 & 45.4 & 71.3 & 60.7 & 123.2 & 49.1 & 73.8 & 61.9 \\
67 & 127.0 & 79.8 & 95.5 & 83.1 & 121.9 & 80.8 & 94.5 & 84.6
\end{tabular}

SAP, systolic arterial pressure DAP, diastolic arterial pressure MAP, mean arterial pressure $\mathrm{HR}$, heart rate 
Table C.4. Raw data for 5 minute average systolic arterial pressure $(\mathrm{mmHg})$ as measured by the finometer for normotensive group in study 2

\begin{tabular}{|c|c|c|c|c|c|c|}
\hline \multirow[b]{2}{*}{ Subject } & \multicolumn{3}{|c|}{ Pre supplementation } & \multicolumn{3}{|c|}{ Post supplementation } \\
\hline & Base & $\begin{array}{l}\text { Mental } \\
\text { Stress }\end{array}$ & Recovery & Base & $\begin{array}{l}\text { Mental } \\
\text { Stress }\end{array}$ & Recovery \\
\hline 1 & 138.5 & 158.3 & 154.3 & 125.0 & 148.3 & 137.4 \\
\hline 2 & 113.5 & 126.4 & 116.4 & 134.9 & 148.8 & 138.3 \\
\hline 3 & 133.9 & 145.4 & 134.9 & 137.9 & 151.6 & 130.1 \\
\hline 4 & 128.8 & 147.6 & 140.6 & 137.2 & 149.4 & 143.6 \\
\hline 5 & 115.5 & 114.2 & 104.1 & 125.1 & 122.2 & 141.9 \\
\hline 6 & 131.3 & 138.2 & 133.9 & 103.5 & 110.5 & 107.1 \\
\hline 7 & 123.4 & 128.7 & 129.6 & 121.6 & 124.9 & 128.0 \\
\hline 8 & 113.8 & 120.6 & 97.4 & 124.1 & 137.0 & 119.4 \\
\hline 9 & 125.7 & 138.0 & 127.0 & 115.6 & 139.0 & 121.8 \\
\hline 10 & 129.0 & 139.0 & 117.1 & 124.3 & 131.1 & 131.3 \\
\hline 11 & 108.4 & 132.6 & 110.6 & 125.3 & 146.3 & 118.0 \\
\hline 12 & 132.9 & 153.4 & 138.6 & 131.8 & 147.9 & 138.1 \\
\hline 13 & 118.4 & 130.3 & 123.5 & 116.0 & 139.2 & 121.5 \\
\hline 14 & 137.1 & 147.2 & 140.0 & 119.8 & 138.5 & -- \\
\hline 15 & 112.8 & 121.1 & 116.6 & 120.0 & 128.8 & 120.1 \\
\hline 16 & 115.5 & 133.8 & 124.6 & 117.5 & 126.4 & 120.8 \\
\hline 17 & 116.8 & 125.6 & 123.9 & 120.1 & 136.9 & 129.3 \\
\hline 18 & 130.9 & 149.5 & 137.5 & 114.9 & 123.2 & 118.5 \\
\hline 19 & 133.8 & 139.7 & 135.7 & 119.0 & 131.7 & 124.0 \\
\hline 20 & 127.7 & 135.2 & 130.9 & 126.1 & 125.6 & 130.5 \\
\hline 21 & 124.0 & 134.0 & 117.1 & 126.7 & 128.4 & 124.9 \\
\hline 22 & 123.1 & 124.0 & 112.7 & 119.1 & 115.3 & 103.3 \\
\hline 23 & 122.1 & 133.3 & 131.0 & 112.1 & 107.9 & 111.5 \\
\hline 24 & 120.6 & 118.8 & 124.9 & 121.4 & 117.3 & 133.8 \\
\hline 25 & 120.5 & 132.5 & 128.1 & 121.0 & 128.0 & 128.4 \\
\hline 26 & 128.5 & 144.7 & 135.3 & 112.2 & 125.6 & 115.5 \\
\hline 27 & 127.1 & 124.3 & 129.7 & 114.9 & 121.3 & 126.7 \\
\hline 28 & 118.6 & 121.5 & 118.6 & 106.5 & 114.2 & 107.8 \\
\hline 29 & 133.8 & 147.7 & 137.9 & 113.4 & 125.7 & 116.0 \\
\hline 30 & 116.0 & 131.7 & 108.0 & 118.5 & 136.6 & 128.1 \\
\hline 31 & 134.5 & 134.8 & 138.7 & 126.9 & 130.6 & 130.6 \\
\hline 32 & 127.3 & 131.7 & 128.8 & 133.6 & 141.1 & 136.6 \\
\hline 33 & 129.6 & 137.2 & 127.8 & 118.7 & 131.8 & 117.4 \\
\hline 34 & 120.6 & 139.1 & 132.3 & 123.4 & 137.2 & 132.9 \\
\hline 35 & 124.4 & 138.8 & 130.5 & 87.8 & 100.7 & 91.6 \\
\hline 36 & 148.0 & 164.6 & 159.1 & 135.6 & 147.5 & 139.0 \\
\hline 37 & 130.2 & 148.8 & 129.0 & 111.4 & 129.5 & 112.5 \\
\hline 38 & 112.0 & 125.1 & 129.1 & 105.2 & 117.8 & 115.0 \\
\hline
\end{tabular}

Missing data point was due to power outage and loss of recovery data. 
Table C.5. Raw data for 5 minute average systolic arterial pressure $(\mathrm{mmHg})$ as measured by the finometer for prehypertensive group in study 2

\begin{tabular}{|c|c|c|c|c|c|c|}
\hline \multirow[b]{2}{*}{ Subject } & \multicolumn{3}{|c|}{ Pre supplementation } & \multicolumn{3}{|c|}{ Post supplementation } \\
\hline & Base & $\begin{array}{c}\text { Mental } \\
\text { Stress }\end{array}$ & Recovery & Base & $\begin{array}{l}\text { Mental } \\
\text { Stress }\end{array}$ & Recovery \\
\hline 39 & 124.8 & 135.7 & 129.3 & 135.8 & 142.1 & 139.2 \\
\hline 40 & 141.2 & 150.1 & 146.9 & 138.2 & 150.1 & 150.0 \\
\hline 41 & 138.6 & 145.0 & 133.2 & 123.2 & 129.1 & 123.4 \\
\hline 42 & 141.5 & 157.1 & 145.4 & 140.9 & 155.9 & 132.2 \\
\hline 43 & 122.6 & -- & 124.5 & -- & -- & -- \\
\hline 44 & 122.1 & 152.1 & 131.4 & 134.1 & 176.5 & 129.6 \\
\hline 45 & 129.7 & 155.6 & 135.1 & 114.1 & 149.1 & 119.6 \\
\hline 46 & 123.7 & 134.8 & 131.0 & 131.8 & 134.3 & 135.4 \\
\hline 47 & 121.7 & 123.2 & 122.2 & 121.6 & 122.4 & 116.3 \\
\hline 48 & 117.7 & 129.4 & 114.5 & 111.6 & 124.6 & 117.5 \\
\hline 49 & 110.1 & 118.2 & 112.4 & 119.6 & 125.3 & 120.5 \\
\hline 50 & 136.4 & 146.4 & 141.7 & 134.7 & 145.9 & 141.2 \\
\hline 51 & 118.8 & 138.9 & 123.8 & 111.4 & 124.2 & 128.1 \\
\hline 52 & 144.2 & 155.9 & 141.7 & 119.8 & 143.6 & 121.0 \\
\hline 53 & 164.9 & 187.8 & 168.2 & 153.2 & 169.4 & 156.0 \\
\hline 54 & 119.8 & 142.2 & 136.1 & 130.1 & 136.5 & 133.0 \\
\hline 55 & 127.9 & 143.0 & 126.6 & 119.8 & 124.2 & 115.4 \\
\hline 56 & 135.0 & 148.8 & 131.2 & 135.5 & 140.8 & 139.1 \\
\hline 57 & 141.5 & 154.7 & 144.7 & 128.6 & 142.5 & 134.8 \\
\hline 58 & 139.1 & 135.8 & 137.0 & 138.5 & 144.1 & 145.4 \\
\hline 59 & 165.5 & 199.9 & 166.1 & 144.1 & 182.7 & 156.7 \\
\hline 60 & 126.7 & 139.3 & 133.0 & 132.1 & 135.2 & 134.7 \\
\hline 61 & 136.6 & 148.0 & 140.5 & -- & -- & -- \\
\hline 62 & 144.0 & 154.5 & 148.2 & 151.3 & 171.6 & 162.6 \\
\hline 63 & 138.9 & 150.4 & 140.4 & 121.2 & 134.3 & 120.1 \\
\hline 64 & 138.4 & 175.1 & 146.7 & 136.1 & 164.7 & 151.4 \\
\hline 65 & 139.3 & 151.7 & 146.8 & 146.8 & 156.0 & 157.2 \\
\hline 66 & 150.6 & 149.4 & 161.4 & 156.0 & 155.8 & 157.3 \\
\hline 67 & 138.5 & 152.8 & 148.3 & 120.1 & 147.1 & 144.1 \\
\hline
\end{tabular}

Missing data points are due to subject withdrawal and equipment error. 
Table C.6. Raw data for 5 minute average diastolic arterial pressure $(\mathrm{mmHg})$ as measured by the finometer for normotensive group in study 2

\begin{tabular}{|c|c|c|c|c|c|c|}
\hline \multirow[b]{2}{*}{ Subject } & \multicolumn{3}{|c|}{ Pre supplementation } & \multicolumn{3}{|c|}{ Post supplementation } \\
\hline & Base & $\begin{array}{l}\text { Mental } \\
\text { Stress }\end{array}$ & Recovery & Base & $\begin{array}{l}\text { Mental } \\
\text { Stress }\end{array}$ & Recovery \\
\hline 1 & 78.3 & 94.4 & 87.0 & 73.5 & 86.7 & 77.8 \\
\hline 2 & 76.4 & 89.1 & 76.2 & 75.9 & 88.3 & 76.8 \\
\hline 3 & 76.7 & 88.1 & 76.5 & 74.8 & 87.9 & 74.4 \\
\hline 4 & 80.3 & 94.5 & 80.5 & 72.7 & 86.6 & 71.3 \\
\hline 5 & 79.8 & 85.2 & 76.1 & 83.0 & 91.2 & 95.2 \\
\hline 6 & 79.9 & 84.6 & 79.7 & 68.5 & 74.5 & 70.1 \\
\hline 7 & 74.9 & 79.3 & 78.2 & 68.8 & 72.2 & 71.3 \\
\hline 8 & 75.4 & 83.9 & 70.6 & 74.3 & 87.2 & 73.2 \\
\hline 9 & 73.7 & 82.1 & 73.6 & 60.9 & 80.1 & 66.7 \\
\hline 10 & 75.0 & 84.3 & 68.9 & 67.8 & 74.8 & 71.0 \\
\hline 11 & 60.7 & 71.9 & 59.9 & 68.3 & 80.8 & 64.4 \\
\hline 12 & 71.3 & 84.4 & 72.4 & 65.0 & 76.7 & 67.4 \\
\hline 13 & 59.8 & 74.3 & 63.1 & 58.0 & 73.2 & 62.4 \\
\hline 14 & 75.9 & 84.3 & 78.5 & 66.8 & 78.9 & -- \\
\hline 15 & 66.2 & 72.6 & 63.5 & 70.8 & 78.4 & 72.4 \\
\hline 16 & 66.5 & 76.1 & 68.4 & 65.2 & 72.0 & 66.1 \\
\hline 17 & 62.1 & 72.5 & 67.0 & 63.3 & 78.6 & 69.3 \\
\hline 18 & 74.0 & 88.2 & 77.8 & 61.3 & 71.2 & 59.9 \\
\hline 19 & 73.8 & 81.8 & 73.0 & 61.8 & 71.5 & 63.8 \\
\hline 20 & 67.4 & 76.2 & 68.5 & 67.4 & 73.9 & 69.2 \\
\hline 21 & 72.2 & 76.2 & 69.9 & 76.4 & 77.6 & 75.6 \\
\hline 22 & 66.8 & 74.2 & 65.2 & 64.4 & 69.4 & 61.0 \\
\hline 23 & 71.1 & 80.5 & 76.2 & 71.7 & 76.6 & 75.5 \\
\hline 24 & 71.6 & 74.9 & 75.0 & 67.6 & 75.4 & 75.8 \\
\hline 25 & 69.5 & 76.0 & 73.9 & 68.9 & 72.8 & 70.6 \\
\hline 26 & 68.7 & 79.6 & 72.3 & 63.1 & 73.4 & 66.2 \\
\hline 27 & 73.4 & 78.9 & 74.5 & 68.4 & 77.8 & 75.5 \\
\hline 28 & 73.5 & 79.1 & 75.0 & 64.8 & 72.6 & 68.1 \\
\hline 29 & 83.2 & 95.4 & 86.3 & 68.2 & 77.8 & 69.5 \\
\hline 30 & 68.5 & 80.9 & 67.4 & 59.9 & 71.9 & 63.7 \\
\hline 31 & 74.7 & 75.9 & 77.8 & 69.3 & 73.6 & 71.0 \\
\hline 32 & 75.0 & 79.4 & 76.1 & 76.3 & 83.5 & 78.4 \\
\hline 33 & 71.5 & 77.8 & 71.6 & 65.2 & 73.8 & 66.6 \\
\hline 34 & 72.8 & 82.4 & 76.7 & 72.6 & 81.4 & 76.7 \\
\hline 35 & 70.4 & 76.9 & 73.4 & 67.9 & 78.4 & 79.5 \\
\hline 36 & 72.4 & 83.1 & 78.5 & 62.1 & 70.0 & 64.3 \\
\hline 37 & 73.4 & 87.8 & 74.3 & 66.9 & 76.3 & 68.0 \\
\hline 38 & 70.5 & 78.9 & 78.8 & 64.5 & 71.1 & 70.5 \\
\hline
\end{tabular}

Missing data point was due to power outage and loss of recovery data. 
Table C.7. Raw data for 5 minute average diastolic arterial pressure $(\mathrm{mmHg})$ as measured by the finometer for prehypertensive group in study 2

\begin{tabular}{|c|c|c|c|c|c|c|}
\hline \multirow[b]{2}{*}{ Subject } & \multicolumn{3}{|c|}{ Pre supplementation } & \multicolumn{3}{|c|}{ Post supplementation } \\
\hline & Base & $\begin{array}{l}\text { Mental } \\
\text { Stress }\end{array}$ & Recovery & Base & $\begin{array}{c}\text { Mental } \\
\text { Stress }\end{array}$ & Recovery \\
\hline 39 & 74.7 & 81.7 & 75.9 & 79.9 & 85.9 & 81.9 \\
\hline 40 & 74.1 & 83.6 & 73.8 & 73.5 & 83.7 & 78.1 \\
\hline 41 & 81.9 & 92.9 & 82.4 & 68.1 & 73.5 & 67.5 \\
\hline 42 & 75.6 & 87.5 & 77.6 & 68.1 & 80.3 & 67.2 \\
\hline 43 & 67.5 & -- & 71.7 & -- & -- & -- \\
\hline 44 & 71.4 & 94.3 & 73.2 & 69.2 & 91.1 & 69.9 \\
\hline 45 & 70.9 & 87.0 & 72.7 & 62.9 & 81.8 & 66.1 \\
\hline 46 & 71.9 & 83.1 & 73.2 & 70.8 & 76.6 & 74.1 \\
\hline 47 & 70.0 & 73.2 & 70.9 & 67.0 & 71.5 & 64.8 \\
\hline 48 & 64.4 & 72.4 & 65.9 & 69.0 & 78.1 & 71.3 \\
\hline 49 & 66.8 & 76.4 & 67.3 & 70.2 & 77.6 & 70.3 \\
\hline 50 & 84.5 & 89.2 & 88.4 & 76.7 & 85.0 & 81.0 \\
\hline 51 & 71.8 & 82.3 & 73.7 & 63.5 & 72.4 & 74.7 \\
\hline 52 & 68.4 & 79.4 & 69.4 & 65.0 & 76.4 & 67.5 \\
\hline 53 & 97.6 & 106.4 & 91.3 & 82.6 & 96.5 & 85.8 \\
\hline 54 & 63.6 & 81.3 & 75.1 & 63.2 & 75.5 & 69.9 \\
\hline 55 & 72.1 & 83.0 & 71.1 & 68.2 & 73.5 & 69.1 \\
\hline 56 & 72.6 & 79.8 & 71.2 & 74.5 & 79.0 & 76.1 \\
\hline 57 & 82.4 & 90.2 & 83.3 & 75.2 & 82.0 & 78.3 \\
\hline 58 & 73.1 & 77.9 & 75.2 & 76.4 & 80.6 & 79.4 \\
\hline 59 & 88.7 & 106.6 & 88.0 & 80.7 & 99.5 & 84.7 \\
\hline 60 & 72.3 & 80.7 & 72.7 & 75.4 & 81.0 & 74.6 \\
\hline 61 & 82.5 & 88.7 & 82.3 & -- & -- & -- \\
\hline 62 & 71.6 & 81.6 & 74.6 & 88.9 & 99.8 & 92.7 \\
\hline 63 & 81.2 & 89.9 & 79.9 & 63.2 & 73.0 & 64.1 \\
\hline 64 & 74.3 & 97.6 & 79.0 & 75.0 & 92.9 & 83.5 \\
\hline 65 & 73.8 & 87.7 & 79.5 & 80.5 & 91.3 & 83.8 \\
\hline 66 & 80.0 & 83.3 & 86.8 & 82.5 & 84.8 & 85.0 \\
\hline 67 & 95.4 & 104.4 & 101.0 & 72.9 & 91.4 & 84.2 \\
\hline
\end{tabular}

Missing data points are due to subject withdrawal and equipment error. 
Table C.8. Raw data for 5 minute average mean arterial pressure $(\mathrm{mmHg})$ as measured by the finometer for normotensive group in study 2

\begin{tabular}{|c|c|c|c|c|c|c|}
\hline \multirow[b]{2}{*}{ Subject } & \multicolumn{3}{|c|}{ Pre supplementation } & \multicolumn{3}{|c|}{ Post supplementation } \\
\hline & Base & $\begin{array}{c}\text { Mental } \\
\text { Stress }\end{array}$ & Recovery & Base & $\begin{array}{l}\text { Mental } \\
\text { Stress }\end{array}$ & Recovery \\
\hline 1 & 97.2 & 116.6 & 109.5 & 89.9 & 106.7 & 97.6 \\
\hline 2 & 90.1 & 104.5 & 90.7 & 97.2 & 113.6 & 99.3 \\
\hline 3 & 98.5 & 110.7 & 98.6 & 96.4 & 110.4 & 94.1 \\
\hline 4 & 102.2 & 118.5 & 105.4 & 100.0 & 115.5 & 100.1 \\
\hline 5 & 93.5 & 97.5 & 88.2 & 99.8 & 104.1 & 113.9 \\
\hline 6 & 97.1 & 103.6 & 98.3 & 81.0 & 88.1 & 83.7 \\
\hline 7 & 91.6 & 97.8 & 97.3 & 87.6 & 92.3 & 92.2 \\
\hline 8 & 89.1 & 98.3 & 81.8 & 90.7 & 106.0 & 89.3 \\
\hline 9 & 91.8 & 104.8 & 93.6 & 79.3 & 103.6 & 87.4 \\
\hline 10 & 97.4 & 109.1 & 90.4 & 88.7 & 95.8 & 94.0 \\
\hline 11 & 76.3 & 93.1 & 77.7 & 86.5 & 103.1 & 82.2 \\
\hline 12 & 96.1 & 115.2 & 99.2 & 87.4 & 105.9 & 92.1 \\
\hline 13 & 81.0 & 97.3 & 87.5 & 78.7 & 95.0 & 85.2 \\
\hline 14 & 101.1 & 105.6 & 100.8 & 86.4 & 104.1 & -- \\
\hline 15 & 82.6 & 91.1 & 81.0 & 90.7 & 98.3 & 90.5 \\
\hline 16 & 86.9 & 103.3 & 93.0 & 87.1 & 97.3 & 89.6 \\
\hline 17 & 82.0 & 93.7 & 88.8 & 84.9 & 102.5 & 93.6 \\
\hline 18 & 99.6 & 116.4 & 104.5 & 83.8 & 94.1 & 83.6 \\
\hline 19 & 95.3 & 105.2 & 95.5 & 81.4 & 93.8 & 84.9 \\
\hline 20 & 88.6 & 98.9 & 91.2 & 87.7 & 94.7 & 91.1 \\
\hline 21 & 89.2 & 94.6 & 86.3 & 92.7 & 94.5 & 92.0 \\
\hline 22 & 87.5 & 92.6 & 83.7 & 81.8 & 84.3 & 75.6 \\
\hline 23 & 87.7 & 99.1 & 94.9 & 83.8 & 87.5 & 87.4 \\
\hline 24 & 85.8 & 90.2 & 90.9 & 84.5 & 91.0 & 96.0 \\
\hline 25 & 90.1 & 99.2 & 96.6 & 88.9 & 95.9 & 93.1 \\
\hline 26 & 89.1 & 103.6 & 94.3 & 81.0 & 93.9 & 84.9 \\
\hline 27 & 91.1 & 95.7 & 93.5 & 84.9 & 94.7 & 94.0 \\
\hline 28 & 89.1 & 95.0 & 91.1 & 80.2 & 88.3 & 84.0 \\
\hline 29 & 101.2 & 116.2 & 105.7 & 85.0 & 97.0 & 87.5 \\
\hline 30 & 82.2 & 99.4 & 80.6 & 77.7 & 93.5 & 83.4 \\
\hline 31 & 95.3 & 98.1 & 99.5 & 91.3 & 96.8 & 94.1 \\
\hline 32 & 94.8 & 100.3 & 95.9 & 97.4 & 105.8 & 100.0 \\
\hline 33 & 90.9 & 98.9 & 91.7 & 83.9 & 95.3 & 85.7 \\
\hline 34 & 90.4 & 104.5 & 97.6 & 90.4 & 102.9 & 96.9 \\
\hline 35 & 92.7 & 101.0 & 96.7 & 76.2 & 86.4 & 84.0 \\
\hline 36 & 102.0 & 116.0 & 110.6 & 89.7 & 99.8 & 92.5 \\
\hline 37 & 89.4 & 108.0 & 90.3 & 80.6 & 92.7 & 81.7 \\
\hline 38 & 84.4 & 95.5 & 96.3 & 78.1 & 87.6 & 85.0 \\
\hline
\end{tabular}

Missing data points are due to subject withdrawal and equipment error. 
Table C.9.Raw data for 5 minute average mean arterial pressure $(\mathrm{mmHg})$ as measured by the finometer for prehypertensive group in study 2

\begin{tabular}{|c|c|c|c|c|c|c|}
\hline \multirow[b]{2}{*}{ Subject } & \multicolumn{3}{|c|}{ Pre supplementation } & \multicolumn{3}{|c|}{ Post supplementation } \\
\hline & Base & $\begin{array}{l}\text { Mental } \\
\text { Stress }\end{array}$ & Recovery & Base & $\begin{array}{c}\text { Mental } \\
\text { Stress }\end{array}$ & Recovery \\
\hline 39 & 90.4 & 101.0 & 93.1 & 97.8 & 105.9 & 100.9 \\
\hline 40 & 97.9 & 110.3 & 100.3 & 94.7 & 110.4 & 102.3 \\
\hline 41 & 100.6 & 111.9 & 100.6 & 86.2 & 92.2 & 86.2 \\
\hline 42 & 97.5 & 115.4 & 101.1 & 90.2 & 106.8 & 88.0 \\
\hline 43 & 86.7 & -- & 92.4 & -- & -- & -- \\
\hline 44 & 90.0 & 114.0 & 93.8 & 91.0 & 118.5 & 90.4 \\
\hline 45 & 90.1 & 111.3 & 93.2 & 80.5 & 105.4 & 85.4 \\
\hline 46 & 90.1 & 102.4 & 93.4 & 91.1 & 97.2 & 95.1 \\
\hline 47 & 83.8 & 89.3 & 85.9 & 82.8 & 87.7 & 80.8 \\
\hline 48 & 79.5 & 90.4 & 81.3 & 81.7 & 93.1 & 86.1 \\
\hline 49 & 80.8 & 91.6 & 82.3 & 87.4 & 95.9 & 87.9 \\
\hline 50 & 102.3 & 111.9 & 107.5 & 94.9 & 106.2 & 101.2 \\
\hline 51 & 85.5 & 101.4 & 89.2 & 77.8 & 88.9 & 92.4 \\
\hline 52 & 88.6 & 101.9 & 88.7 & 82.1 & 96.5 & 85.1 \\
\hline 53 & 114.2 & 139.0 & 119.0 & 102.7 & 121.9 & 107.4 \\
\hline 54 & 81.1 & 102.6 & 96.0 & 82.7 & 96.2 & 90.4 \\
\hline 55 & 90.4 & 103.4 & 90.5 & 83.1 & 88.1 & 83.3 \\
\hline 56 & 96.7 & 106.0 & 95.0 & 97.3 & 102.1 & 100.7 \\
\hline 57 & 104.4 & 114.9 & 107.0 & 95.4 & 105.7 & 100.6 \\
\hline 58 & 95.5 & 101.2 & 98.2 & 98.6 & 105.0 & 103.3 \\
\hline 59 & 120.4 & 146.7 & 119.4 & 108.5 & 135.8 & 115.4 \\
\hline 60 & 91.4 & 103.4 & 93.9 & 92.7 & 101.0 & 94.4 \\
\hline 61 & 101.5 & 111.6 & 102.9 & -- & -- & -- \\
\hline 62 & 93.9 & 106.1 & 97.6 & 109.8 & 124.7 & 116.5 \\
\hline 63 & 100.9 & 111.8 & 100.9 & 82.2 & 94.4 & 83.4 \\
\hline 64 & 96.9 & 127.4 & 103.6 & 95.1 & 119.1 & 106.3 \\
\hline 65 & 94.5 & 110.7 & 102.0 & 102.1 & 115.0 & 108.1 \\
\hline 66 & 98.0 & 103.4 & 106.9 & 100.8 & 105.9 & 104.5 \\
\hline 67 & 110.9 & 122.7 & 118.4 & 89.2 & 111.7 & 105.1 \\
\hline
\end{tabular}

Missing data points are due to subject withdrawal and equipment error. 
Table C.10. Raw data for 5 minute average heart rate (beats per minute) as measured by the finometer for normotensive group in study 2

\begin{tabular}{|c|c|c|c|c|c|c|}
\hline \multirow[b]{2}{*}{ Subject } & \multicolumn{3}{|c|}{ Pre supplementation } & \multicolumn{3}{|c|}{ Post supplementation } \\
\hline & Base & $\begin{array}{l}\text { Mental } \\
\text { Stress }\end{array}$ & Recovery & Base & $\begin{array}{l}\text { Mental } \\
\text { Stress }\end{array}$ & Recovery \\
\hline 1 & 67.7 & 94.9 & 75.5 & 66.9 & 93.5 & 73.1 \\
\hline 2 & 64.5 & 75.7 & 65.1 & 63.0 & 75.2 & 61.7 \\
\hline 3 & 74.6 & 126.2 & 79.7 & 77.7 & 115.5 & 71.4 \\
\hline 4 & 71.3 & 112.1 & 67.4 & 66.1 & 109.7 & 58.8 \\
\hline 5 & 65.7 & 98.1 & 65.1 & 78.4 & 109.2 & 73.9 \\
\hline 6 & 89.1 & 96.3 & 86.1 & 87.3 & 94.1 & 87.9 \\
\hline 7 & 62.0 & 70.6 & 60.3 & 61.0 & 70.0 & 56.5 \\
\hline 8 & 60.6 & 69.1 & 60.9 & 62.1 & 75.3 & 58.9 \\
\hline 9 & 65.3 & 83.8 & 64.9 & 60.8 & 76.9 & 64.6 \\
\hline 10 & 57.1 & 70.6 & 56.1 & 67.5 & 78.8 & 65.8 \\
\hline 11 & 51.1 & 73.8 & 50.4 & 63.9 & 83.8 & 57.1 \\
\hline 12 & 58.0 & 91.2 & 58.1 & 55.8 & 76.1 & 56.6 \\
\hline 13 & 49.6 & 70.0 & 51.3 & 52.9 & 71.3 & 52.5 \\
\hline 14 & 78.6 & 102.4 & 74.5 & 77.8 & 107.3 & -- \\
\hline 15 & 73.4 & 100.5 & 71.9 & 67.1 & 83.2 & 61.7 \\
\hline 16 & 64.5 & 80.2 & 64.5 & 63.6 & 75.2 & 63.9 \\
\hline 17 & 75.6 & 91.7 & 74.0 & 73.2 & 97.1 & 68.6 \\
\hline 18 & 80.4 & 103.3 & 76.5 & 82.8 & 99.0 & 77.0 \\
\hline 19 & 63.6 & 72.8 & 65.5 & 62.0 & 68.5 & 64.9 \\
\hline 20 & 64.1 & 80.6 & 63.3 & 63.3 & 81.3 & 58.9 \\
\hline 21 & 68.8 & 73.5 & 69.5 & 72.3 & 76.5 & 68.7 \\
\hline 22 & 61.6 & 76.4 & 59.2 & 70.4 & 83.6 & 69.9 \\
\hline 23 & 48.8 & 74.8 & 49.5 & 59.2 & 86.1 & 56.7 \\
\hline 24 & 47.7 & 72.9 & 48.6 & 49.4 & 78.3 & 48.5 \\
\hline 25 & 58.4 & 76.3 & 59.2 & 70.7 & 91.2 & 70.7 \\
\hline 26 & 66.7 & 91.8 & 67.5 & 60.0 & 78.4 & 62.2 \\
\hline 27 & 62.0 & 92.6 & 61.7 & 48.0 & 87.7 & 57.6 \\
\hline 28 & 52.9 & 69.9 & 53.5 & 56.6 & 76.5 & 61.6 \\
\hline 29 & 58.0 & 74.3 & 57.8 & 62.4 & 81.0 & 61.8 \\
\hline 30 & 58.2 & 75.7 & 57.4 & 62.0 & 77.2 & 65.8 \\
\hline 31 & 65.2 & 73.7 & 64.7 & 64.5 & 73.1 & 65.2 \\
\hline 32 & 57.4 & 70.3 & 61.2 & 56.2 & 63.4 & 57.3 \\
\hline 33 & 60.8 & 68.0 & 61.6 & 63.4 & 69.0 & 62.6 \\
\hline 34 & 64.0 & 88.1 & 67.0 & 58.1 & 77.7 & 64.1 \\
\hline 35 & 60.2 & 62.0 & 59.7 & 69.5 & 68.2 & 66.2 \\
\hline 36 & 48.3 & 56.5 & 49.4 & 50.6 & 54.6 & 51.6 \\
\hline 37 & 65.4 & 84.0 & 63.9 & 72.4 & 92.9 & 66.3 \\
\hline 38 & 82.6 & 93.2 & 84.2 & 84.0 & 94.4 & 84.9 \\
\hline
\end{tabular}

Missing data points are due to subject withdrawal and equipment error. 
Table C.11. Raw data for 5 minute average heart rate (beats per minute) as measured by the finometer for prehypertensive group in study 2

$$
\text { Pre supplementation }
$$

\begin{tabular}{|c|c|c|c|}
\hline Subject & Base & $\begin{array}{l}\text { Mental } \\
\text { Stress }\end{array}$ & Recovery \\
\hline 39 & 51.2 & 64.8 & 53.4 \\
\hline 40 & 71.0 & 87.9 & 67.7 \\
\hline 41 & 72.0 & 83.7 & 76.0 \\
\hline 42 & 67.4 & 84.3 & 65.0 \\
\hline 43 & 49.6 & 73.6 & 54.0 \\
\hline 44 & 71.2 & 128.9 & 80.9 \\
\hline 45 & 80.3 & 114.2 & 83.2 \\
\hline 46 & 62.0 & 73.8 & 64.6 \\
\hline 47 & 71.9 & 92.7 & 78.8 \\
\hline 48 & 52.9 & 70.6 & 58.0 \\
\hline 49 & 77.4 & 88.9 & 77.4 \\
\hline 50 & 69.1 & 86.6 & 70.0 \\
\hline 51 & 79.1 & 86.2 & 78.6 \\
\hline 52 & 63.5 & 76.1 & 60.9 \\
\hline 53 & 67.1 & 96.6 & 65.8 \\
\hline 54 & 59.0 & 72.3 & 61.7 \\
\hline 55 & 69.8 & 80.1 & 74.7 \\
\hline 56 & 62.9 & 65.2 & 62.9 \\
\hline 57 & 81.1 & 100.2 & 79.1 \\
\hline 58 & 68.1 & 79.6 & 65.9 \\
\hline 59 & 76.8 & 92.7 & 77.6 \\
\hline 60 & 61.2 & 82.2 & 65.1 \\
\hline 61 & 69.0 & 80.6 & 67.8 \\
\hline 62 & 65.1 & 79.5 & 64.0 \\
\hline 63 & 72.8 & 92.2 & 77.2 \\
\hline 64 & 69.2 & 91.2 & 72.4 \\
\hline 65 & 66.8 & 88.4 & 67.9 \\
\hline 66 & 55.4 & 78.5 & 59.3 \\
\hline 67 & 63.4 & 88.2 & 72.3 \\
\hline
\end{tabular}

Post supplementation

$\begin{array}{ccc}\text { Base } & \text { Mental } & \text { Recovery } \\ & \text { Stress } & \\ 56.3 & 66.4 & 57.5 \\ 56.9 & 83.4 & 57.2 \\ 71.2 & 80.8 & 74.1 \\ 63.0 & 74.5 & 60.0 \\ -- & -- & -- \\ 89.3 & 132.0 & 92.9 \\ 67.0 & 96.2 & 76.3 \\ 60.4 & 71.4 & 62.9 \\ 73.0 & 91.4 & 76.7 \\ 58.0 & 73.9 & 62.0 \\ 63.0 & 74.6 & 59.4 \\ 57.2 & 71.3 & 59.9 \\ 74.4 & 82.0 & 78.3 \\ 67.1 & 78.6 & 66.0 \\ 70.9 & 89.5 & 67.8 \\ 57.8 & 77.8 & 66.2 \\ 69.8 & 71.9 & 69.0 \\ 64.1 & 65.2 & 66.0 \\ 72.5 & 89.7 & 72.4 \\ 70.9 & 75.1 & 65.6 \\ 69.7 & 95.9 & 73.1 \\ 50.1 & 73.1 & 54.0 \\ -- & -- & -- \\ 78.7 & 92.1 & 76.3 \\ 66.7 & 86.0 & 70.9 \\ 72.6 & 95.6 & 75.9 \\ 65.8 & 88.5 & 65.4 \\ 52.8 & 73.5 & 50.6 \\ 61.8 & 88.9 & 68.0\end{array}$

Missing data points are due to subject withdrawal and equipment error. 
Table C.12. Raw data for 5 minute average MSNA (burst per minute) for normotensive group in study 2

\begin{tabular}{|c|c|c|c|c|c|c|}
\hline \multirow[b]{2}{*}{ Subject } & \multicolumn{3}{|c|}{ Pre supplementation } & \multicolumn{3}{|c|}{ Post supplementation } \\
\hline & Base & $\begin{array}{l}\text { Mental } \\
\text { Stress }\end{array}$ & Recovery & Base & $\begin{array}{l}\text { Mental } \\
\text { Stress }\end{array}$ & Recovery \\
\hline 1 & 8.6 & 12.4 & 6.4 & 8.2 & 18.0 & 15.4 \\
\hline 2 & 5.2 & -- & -- & 13.6 & 20.5 & 19.8 \\
\hline 3 & 2.6 & -- & -- & 12.6 & 17.4 & 17.2 \\
\hline 4 & 16.6 & 25.2 & 27.8 & 11.6 & 14.8 & 15.4 \\
\hline 5 & -- & -- & -- & -- & -- & -- \\
\hline 6 & 6.2 & -- & -- & -- & -- & -- \\
\hline 7 & 13.8 & 18.2 & 20.2 & -- & -- & -- \\
\hline 8 & 7.2 & 11.4 & 17.0 & 3.8 & 10.6 & -- \\
\hline 9 & 20.0 & -- & -- & 20.2 & 24.2 & 28.2 \\
\hline 10 & 10.4 & 10.8 & 18.0 & 4.2 & 5.4 & 4.6 \\
\hline 11 & 6.6 & 12.6 & 11.6 & 2.0 & -- & -- \\
\hline 12 & 12.7 & -- & -- & 12.4 & 16.0 & 15.8 \\
\hline 13 & 1.0 & 19.4 & -- & 6.4 & 12.4 & 4.4 \\
\hline 14 & 8.8 & 13.2 & 10.8 & 3.6 & 10.8 & -- \\
\hline 15 & 13.4 & 18.4 & 24.6 & 16.3 & -- & -- \\
\hline 16 & 10.8 & 7.0 & 8.2 & 7.6 & 12.2 & 9.0 \\
\hline 17 & 7.6 & -- & -- & 7.2 & 9.8 & 9.0 \\
\hline 18 & 8.2 & -- & -- & 5.0 & -- & -- \\
\hline 19 & 10.8 & -- & -- & 12.2 & 19.8 & 10.2 \\
\hline 20 & 6.2 & 24.2 & 7.8 & 4.8 & 14.0 & 6.4 \\
\hline 21 & 15.6 & 21.2 & 19.2 & 9.4 & 12.8 & 15.2 \\
\hline 22 & 9.2 & 21.0 & 16.4 & 28.4 & 42.6 & 32.6 \\
\hline 23 & 4.0 & 5.8 & 3.2 & 7.8 & 11.0 & 12.6 \\
\hline 24 & -- & -- & -- & 4.6 & 9.0 & 8.8 \\
\hline 25 & 6.6 & -- & 10.2 & 8.0 & -- & -- \\
\hline 26 & -- & -- & -- & 12.4 & 18.6 & 26.4 \\
\hline 27 & 8.8 & 11.8 & 13.2 & 5.6 & 11.2 & 12.0 \\
\hline 28 & 16.0 & 17.0 & 28.0 & 9.2 & 15.2 & 14.8 \\
\hline 29 & 10.6 & -- & -- & 6.4 & -- & -- \\
\hline 30 & 4.0 & 14.0 & 9.2 & 14.0 & 28.0 & 17.2 \\
\hline 31 & 9.0 & 10.0 & 9.8 & 16.0 & 22.0 & 15.0 \\
\hline 32 & 10.3 & -- & -- & 3.7 & 6.2 & 6.0 \\
\hline 33 & 29.4 & 37.2 & 29.6 & 13.0 & 23.2 & 23.4 \\
\hline 34 & 8.2 & -- & -- & 8.8 & -- & -- \\
\hline 35 & 22.4 & 28.8 & 20.4 & 38.4 & 34.7 & -- \\
\hline 36 & 10.6 & 19.8 & 16.8 & 18.2 & 29.0 & -- \\
\hline 37 & 6.8 & 16.6 & 21.2 & 9.6 & 19.6 & 10.2 \\
\hline 38 & 24.6 & 26.8 & 31.0 & 20.6 & 30.4 & 29.0 \\
\hline
\end{tabular}

Missing MSNA data are due to either failure to locate the nerve or loss of nerve during recording 
Table C.13. Raw data for 5 minute average MSNA (burst per minute) for prehypertensive group in study 2

\begin{tabular}{|c|c|c|c|c|c|c|}
\hline \multirow[b]{2}{*}{ Subject } & \multicolumn{3}{|c|}{ Pre supplementation } & \multicolumn{3}{|c|}{ Post supplementation } \\
\hline & Base & $\begin{array}{c}\text { Mental } \\
\text { Stress }\end{array}$ & Recovery & Base & $\begin{array}{c}\text { Mental } \\
\text { Stress }\end{array}$ & Recovery \\
\hline 39 & 7.0 & -- & -- & 19.2 & 20.4 & 22.2 \\
\hline 40 & 27.0 & 31.7 & -- & 13.0 & 19.4 & 24.8 \\
\hline 41 & 25.2 & 29.2 & 28.8 & 11.0 & 18.4 & 11.4 \\
\hline 42 & -- & -- & -- & 3.0 & -- & -- \\
\hline 43 & 3.4 & -- & -- & 4.8 & 7.0 & 11.7 \\
\hline 44 & 8.2 & 19.0 & 23.4 & 3.4 & 10.0 & 6.4 \\
\hline 45 & 3.8 & 9.6 & 6.2 & 3.0 & -- & -- \\
\hline 46 & 2.8 & 6.2 & 6.0 & -- & -- & -- \\
\hline 47 & 7.2 & -- & -- & 10.2 & 13.2 & 12.8 \\
\hline 48 & 18.0 & -- & -- & 27.0 & 22.8 & 32.0 \\
\hline 49 & 7.0 & -- & -- & -- & -- & -- \\
\hline 50 & 10.8 & 12.6 & 15.2 & 14.2 & 14.4 & 20.4 \\
\hline 51 & 17.0 & 11.8 & 17.8 & 7.0 & -- & -- \\
\hline 52 & 6.4 & 20.3 & -- & 13.2 & 16.0 & 14.6 \\
\hline 53 & 21.4 & -- & -- & 18.0 & -- & -- \\
\hline 54 & 4.8 & 13.6 & 10.0 & 8.8 & -- & -- \\
\hline 55 & 26.8 & 28.6 & 38.2 & 18.4 & 21.0 & -- \\
\hline 56 & 9.3 & -- & 15.2 & 4.2 & 9.6 & 4.6 \\
\hline 57 & 2.6 & 3.6 & 7.0 & 7.4 & 2.6 & 8.2 \\
\hline 58 & 13.2 & -- & -- & 19.2 & 27.2 & 15.6 \\
\hline 59 & 9.4 & -- & -- & -- & -- & -- \\
\hline 60 & 9.6 & 18.2 & 15.6 & 10.0 & 7.0 & 14.0 \\
\hline 61 & 9.6 & 13.2 & 15.0 & -- & -- & -- \\
\hline 62 & 17.0 & 34.3 & 30.0 & -- & -- & -- \\
\hline 63 & 14.0 & 15.3 & 21.0 & 6.2 & 8.4 & 6.6 \\
\hline 64 & 16.4 & 27.0 & 27.2 & -- & -- & -- \\
\hline 65 & 19.0 & 30.8 & 27.2 & -- & -- & -- \\
\hline 66 & 10.8 & -- & -- & 3.8 & -- & -- \\
\hline 67 & 7.4 & -- & 15.6 & 7.8 & 11.2 & 6.0 \\
\hline
\end{tabular}

Missing MSNA data are due to either failure to locate the nerve or loss of nerve during recording 
Table C.14. Raw data for 5 minute average MSNA (burst per 100 heart beats) for normotensive group in study 2

\begin{tabular}{|c|c|c|c|c|c|c|}
\hline \multirow[b]{2}{*}{ Subject } & \multicolumn{3}{|c|}{ Pre supplementation } & \multicolumn{3}{|c|}{ Post supplementation } \\
\hline & Base & $\begin{array}{l}\text { Mental } \\
\text { Stress }\end{array}$ & Recovery & Base & $\begin{array}{l}\text { Mental } \\
\text { Stresss }\end{array}$ & Recovery \\
\hline 1 & 12.7 & 13.2 & 8.4 & 12.3 & 19.4 & 21.2 \\
\hline 2 & 8.1 & -- & -- & 21.7 & 27.2 & 32.3 \\
\hline 3 & 3.5 & -- & -- & 17.0 & 15.0 & 24.8 \\
\hline 4 & 23.4 & 22.8 & 41.6 & 17.7 & 13.6 & 26.2 \\
\hline 5 & -- & -- & -- & -- & -- & -- \\
\hline 6 & 7.0 & -- & -- & -- & -- & -- \\
\hline 7 & 22.5 & 26.0 & 33.8 & -- & -- & -- \\
\hline 8 & 12.0 & 16.6 & 28.7 & 6.2 & 14.6 & -- \\
\hline 9 & 30.8 & -- & -- & 33.6 & 31.7 & 43.8 \\
\hline 10 & 18.4 & 15.4 & 32.1 & 6.3 & 6.9 & 7.0 \\
\hline 11 & 12.9 & 17.9 & 23.1 & 3.1 & -- & -- \\
\hline 12 & 22.0 & -- & -- & 22.2 & 21.1 & 28.1 \\
\hline 13 & 2.0 & 28.1 & -- & 12.2 & 17.5 & 8.5 \\
\hline 14 & 11.3 & 13.2 & 14.6 & 4.6 & 10.3 & -- \\
\hline 15 & 18.5 & 18.3 & 34.9 & 24.7 & -- & -- \\
\hline 16 & 16.8 & 9.0 & 12.9 & 12.0 & 16.4 & 14.1 \\
\hline 17 & 10.2 & -- & -- & 10.0 & 10.2 & 13.4 \\
\hline 18 & 11.1 & -- & -- & 6.1 & -- & -- \\
\hline 19 & 17.2 & -- & -- & 19.8 & 29.0 & 15.9 \\
\hline 20 & 9.7 & 30.2 & 12.4 & 7.8 & 17.3 & 11.0 \\
\hline 21 & 23.1 & 29.1 & 28.3 & 13.4 & 17.0 & 22.4 \\
\hline 22 & 15.0 & 27.7 & 28.0 & 40.6 & 51.3 & 46.8 \\
\hline 23 & 8.3 & 7.8 & 6.5 & 13.3 & 12.9 & 22.4 \\
\hline 24 & -- & -- & -- & 9.3 & 11.8 & 18.0 \\
\hline 25 & 11.5 & -- & 17.3 & 11.4 & -- & -- \\
\hline 26 & -- & -- & -- & 20.7 & 24.1 & 42.5 \\
\hline 27 & 14.2 & 12.8 & 21.5 & 11.8 & 12.8 & 21.2 \\
\hline 28 & 30.4 & 24.4 & 52.8 & 16.4 & 19.9 & 24.2 \\
\hline 29 & 18.4 & -- & -- & 10.3 & -- & -- \\
\hline 30 & 7.0 & 18.6 & 16.1 & 22.7 & 36.4 & 26.4 \\
\hline 31 & 14.3 & 13.7 & 15.5 & 24.9 & 30.1 & 23.2 \\
\hline 32 & 18.0 & -- & -- & 6.7 & 9.8 & 10.5 \\
\hline 33 & 48.6 & 54.6 & 48.2 & 20.6 & 33.9 & 37.4 \\
\hline 34 & 12.9 & -- & -- & 15.7 & -- & -- \\
\hline 35 & 37.3 & 46.8 & 34.2 & 55.2 & 50.7 & -- \\
\hline 36 & 22.0 & 35.1 & 34.1 & 36.2 & 51.8 & -- \\
\hline 37 & 10.4 & 20.1 & 33.3 & 13.5 & 21.7 & 15.5 \\
\hline 38 & 29.8 & 28.6 & 36.9 & 24.5 & 32.3 & 34.3 \\
\hline
\end{tabular}

Missing MSNA data are due to either failure to locate the nerve or loss of nerve during recording 
Table C.15. Raw data for 5 minute average MSNA (burst per 100 heart beats) for prehypertensive group in study 2

\begin{tabular}{|c|c|c|c|c|c|c|}
\hline \multirow[b]{2}{*}{ Subject } & \multicolumn{3}{|c|}{ Pre supplementation } & \multicolumn{3}{|c|}{ Post supplementation } \\
\hline & Base & $\begin{array}{c}\text { Mental } \\
\text { Stress }\end{array}$ & Recovery & Base & $\begin{array}{l}\text { Mental } \\
\text { Stress }\end{array}$ & Recovery \\
\hline 39 & 13.7 & -- & -- & 34.2 & 30.9 & 38.7 \\
\hline 40 & 38.7 & 36.8 & -- & 23.2 & 23.4 & 43.4 \\
\hline 41 & 35.0 & 35.1 & 38.0 & 15.5 & 22.9 & 15.4 \\
\hline 42 & -- & -- & -- & 4.9 & -- & -- \\
\hline 43 & 7.0 & -- & -- & 9.0 & 8.7 & 18.8 \\
\hline 44 & 11.7 & 14.2 & 29.1 & 3.8 & 7.8 & 7.0 \\
\hline 45 & 4.8 & 8.4 & 7.4 & 4.5 & -- & -- \\
\hline 46 & 4.6 & 8.4 & 9.4 & -- & -- & -- \\
\hline 47 & 10.5 & -- & -- & 14.1 & 14.5 & 16.9 \\
\hline 48 & 34.2 & -- & -- & 47.0 & 31.2 & 51.8 \\
\hline 49 & 9.1 & -- & -- & -- & -- & -- \\
\hline 50 & 15.7 & 14.5 & 21.8 & 25.0 & 20.3 & 34.2 \\
\hline 51 & 21.7 & 13.8 & 22.7 & 9.4 & -- & -- \\
\hline 52 & 10.1 & 26.1 & -- & 19.8 & 20.6 & 22.3 \\
\hline 53 & 32.1 & -- & -- & 26.0 & -- & -- \\
\hline 54 & 8.2 & 19.1 & 16.4 & 15.4 & -- & -- \\
\hline 55 & 38.6 & 35.7 & 51.2 & 26.5 & 29.0 & -- \\
\hline 56 & 14.6 & -- & 24.2 & 6.6 & 14.9 & 7.1 \\
\hline 57 & 3.2 & 3.5 & 8.9 & 10.3 & 2.8 & 11.4 \\
\hline 58 & 19.5 & -- & -- & 27.4 & 36.6 & 24.1 \\
\hline 59 & 12.3 & -- & -- & -- & -- & -- \\
\hline 60 & 16.1 & 22.7 & 24.2 & 20.5 & 9.6 & 27.3 \\
\hline 61 & 14.0 & 16.6 & 22.2 & -- & -- & - \\
\hline 62 & 26.5 & 44.0 & 47.2 & -- & -- & -- \\
\hline 63 & 19.3 & 16.5 & 27.3 & 9.5 & 9.9 & 9.3 \\
\hline 64 & 23.9 & 30.2 & 38.0 & -- & -- & -- \\
\hline 65 & 28.6 & 35.1 & 40.2 & -- & -- & -- \\
\hline 66 & 19.8 & -- & -- & 7.4 & -- & -- \\
\hline 67 & 11.8 & -- & 21.8 & 12.7 & 12.3 & 8.9 \\
\hline
\end{tabular}

Missing MSNA data are due to either failure to locate the nerve or loss of nerve during recording 
Table C.16. Raw data for 5 minute average total MSNA (arbitrary units) for normotensive group in study 2

\begin{tabular}{|c|c|c|c|c|c|c|}
\hline \multirow[b]{2}{*}{ Subject } & \multicolumn{3}{|c|}{ Pre supplementation } & \multicolumn{3}{|c|}{ Post supplementation } \\
\hline & Base & $\begin{array}{l}\text { Mental } \\
\text { Stress }\end{array}$ & Recovery & Base & $\begin{array}{c}\text { Mental } \\
\text { Stress }\end{array}$ & Recovery \\
\hline 1 & 4665.4 & 12323.9 & 4687.6 & 3820.1 & 11286.9 & 7339.5 \\
\hline 2 & 2704.6 & -- & -- & 6662.3 & 18142.9 & 12903.2 \\
\hline 3 & 2265.5 & -- & -- & 6584.6 & 6934.1 & 5706.1 \\
\hline 4 & 6730.0 & 24173.5 & 19916.7 & 5967.3 & 11408.5 & 7038.5 \\
\hline 5 & -- & -- & -- & -- & -- & -- \\
\hline 6 & 3065.7 & -- & -- & -- & -- & -- \\
\hline 7 & 5554.5 & 7736.9 & 7285.6 & -- & -- & -- \\
\hline 8 & 3667.7 & 7267.5 & 7422.4 & 224.6 & 615.8 & -- \\
\hline 9 & 9606.9 & -- & -- & 10124.0 & 12093.9 & 10585.7 \\
\hline 10 & 5046.7 & 9533.7 & 8090.3 & 2241.0 & -- & 2193.5 \\
\hline 11 & 3346.8 & 12917.3 & 9870.0 & 935.8 & -- & -- \\
\hline 12 & 4938.3 & -- & -- & 5586.5 & 6831.5 & 6436.7 \\
\hline 13 & 800.5 & 24062.9 & -- & 3496.2 & 11280.5 & 3698.6 \\
\hline 14 & 4711.7 & 14853.2 & 9564.4 & 1152.1 & 6194.4 & -- \\
\hline 15 & 5748.1 & 10092.1 & 12825.4 & 7294.1 & -- & -- \\
\hline 16 & 4574.9 & 4998.9 & 2618.6 & 3289.1 & 7299.5 & 3498.3 \\
\hline 17 & 2571.1 & -- & -- & 2546.9 & 7136.8 & 3388.1 \\
\hline 18 & 3098.4 & -- & -- & 1817.0 & -- & -- \\
\hline 19 & 4260.0 & -- & -- & 5683.6 & 14405.7 & 4319.5 \\
\hline 20 & 3143.7 & 16129.0 & 2970.2 & 2640.1 & 9107.4 & 3399.0 \\
\hline 21 & 7878.0 & 8252.9 & 6569.8 & 5130.1 & 10731.5 & 6840.0 \\
\hline 22 & 2350.2 & 7873.3 & 3258.2 & 14351.3 & 28460.9 & 17619.9 \\
\hline 23 & 1921.1 & 4301.4 & 1921.2 & 3973.7 & 9161.5 & 7003.4 \\
\hline 24 & -- & -- & -- & 1963.6 & 7147.8 & 3867.8 \\
\hline 25 & 2973.5 & -- & 4466.0 & 3614.3 & -- & -- \\
\hline 26 & -- & -- & -- & 6025.5 & 6699.9 & 8232.9 \\
\hline 27 & 4209.9 & 6218.5 & 4504.8 & 3132.7 & 4552.0 & 3263.5 \\
\hline 28 & 6464.1 & 13705.9 & 11897.5 & 4365.2 & 6656.8 & 5332.4 \\
\hline 29 & 6906.8 & -- & -- & 4188.6 & -- & -- \\
\hline 30 & 1286.6 & 8854.8 & 3526.3 & 8400.2 & 26312.9 & 9876.6 \\
\hline 31 & 5684.6 & 5717.6 & 6768.9 & 5462.7 & 8503.3 & 5482.3 \\
\hline 32 & 5504.7 & -- & -- & 1591.1 & 3297.7 & 3265.0 \\
\hline 33 & -- & -- & -- & 5099.0 & 12838.4 & 14463.3 \\
\hline 34 & 3072.2 & -- & -- & 3417.9 & -- & -- \\
\hline 35 & 10041.5 & 10316.6 & 5727.2 & 16248.8 & 12310.7 & -- \\
\hline 36 & 2843.0 & 7800.8 & 3868.1 & 5269.1 & 8711.6 & -- \\
\hline 37 & 3140.3 & 18631.6 & 12948.5 & 4424.3 & 28032.8 & 6547.7 \\
\hline 38 & 12032.9 & 15429.1 & 17749.6 & 7990.9 & -- & -- \\
\hline
\end{tabular}

Missing MSNA data are due to either failure to locate the nerve or loss of nerve during recording 
Table C.17. Raw data for 5 minute average total MSNA (arbitrary units) for prehypertensive group in study 2

\begin{tabular}{|c|c|c|c|c|c|c|}
\hline \multirow[b]{2}{*}{ Subject } & \multicolumn{3}{|c|}{ Pre supplementation } & \multicolumn{3}{|c|}{ Post supplementation } \\
\hline & Base & $\begin{array}{l}\text { Mental } \\
\text { Stress }\end{array}$ & Recovery & Base & $\begin{array}{l}\text { Mental } \\
\text { Stress }\end{array}$ & Recovery \\
\hline 39 & 3919.4 & -- & -- & 5162.6 & 11610.4 & 5027.6 \\
\hline 40 & 15174.3 & 25679.3 & -- & 6096.6 & 10364.8 & 12806.8 \\
\hline 41 & 9522.4 & 26003.6 & 9210.4 & 6195.4 & 15282.2 & 5933.5 \\
\hline 42 & -- & -- & -- & 1665.6 & -- & -- \\
\hline 43 & 2048.1 & -- & -- & 2345.6 & 4604.0 & 3760.4 \\
\hline 44 & -- & -- & -- & 351.2 & 13771.8 & 3820.4 \\
\hline 45 & 2427.1 & 7951.7 & 2560.0 & 1884.8 & -- & -- \\
\hline 46 & 1349.4 & 4434.6 & 4417.1 & -- & -- & -- \\
\hline 47 & 2875.2 & -- & -- & 6176.8 & 15374.2 & 9785.8 \\
\hline 48 & 9547.2 & -- & -- & 14516.6 & 15627.3 & 16769.1 \\
\hline 49 & 3173.9 & -- & -- & -- & -- & -- \\
\hline 50 & 4089.3 & 14199.4 & 6910.5 & 6542.0 & 4607.5 & 6222.0 \\
\hline 51 & 8623.5 & 9246.8 & 9207.2 & 3472.3 & -- & -- \\
\hline 52 & 4365.1 & 13956.6 & -- & -- & -- & -- \\
\hline 53 & 10038.6 & -- & -- & 9243.8 & -- & -- \\
\hline 54 & 2691.8 & 10415.2 & 5714.0 & 3574.1 & -- & -- \\
\hline 55 & 11721.3 & 20335.4 & 26744.4 & 6887.0 & 8873.3 & -- \\
\hline 56 & 3117.0 & -- & 6244.8 & 1545.2 & 3976.4 & 1160.3 \\
\hline 57 & 1017.4 & -- & -- & 2139.2 & 695.2 & 1795.5 \\
\hline 58 & 6540.3 & -- & -- & 9107.3 & -- & -- \\
\hline 59 & 2077.1 & -- & -- & -- & -- & -- \\
\hline 60 & 5505.9 & 10479.3 & 8407.0 & 3909.1 & 2175.1 & 3979.0 \\
\hline 61 & 5691.5 & 11318.9 & 9139.0 & -- & -- & -- \\
\hline 62 & 7047.0 & 13083.2 & 15772.5 & -- & -- & -- \\
\hline 63 & 8480.7 & 19125.3 & 18473.9 & 2315.6 & 4114.8 & 3247.8 \\
\hline 64 & 8304.4 & 39597.7 & 23942.1 & -- & -- & -- \\
\hline 65 & 9208.9 & 24776.4 & 14069.5 & -- & -- & -- \\
\hline 66 & 5690.6 & -- & -- & 2259.4 & -- & -- \\
\hline 67 & 3395.2 & -- & 10171.7 & 4884.4 & 7990.8 & 2819.0 \\
\hline
\end{tabular}

Missing MSNA data are due to either failure to locate the nerve or loss of nerve during recording 
Table C.18. Raw data for 5 minute average forearm blood flow for normotensive group in study 2

\begin{tabular}{|c|c|c|c|c|c|c|}
\hline \multirow[b]{2}{*}{ Subject } & \multicolumn{3}{|c|}{ Pre supplementation } & \multicolumn{3}{|c|}{ Post supplementation } \\
\hline & Base & $\begin{array}{l}\text { Mental } \\
\text { Stress }\end{array}$ & Recovery & Base & $\begin{array}{l}\text { Mental } \\
\text { Stress }\end{array}$ & Recovery \\
\hline 1 & 5.80 & 9.77 & 6.78 & 5.21 & 7.64 & 7.53 \\
\hline 2 & 3.31 & 4.26 & 3.53 & 2.76 & 4.47 & 2.88 \\
\hline 3 & 4.10 & 10.50 & 4.03 & 3.84 & 6.43 & 2.89 \\
\hline 4 & 3.55 & 9.23 & 3.35 & 5.21 & 14.98 & 3.94 \\
\hline 5 & 2.84 & 8.17 & 3.25 & 5.66 & 9.03 & 4.42 \\
\hline 6 & 2.94 & 3.59 & 2.69 & 3.61 & 2.90 & 2.74 \\
\hline 7 & 2.07 & 4.49 & 2.04 & 3.10 & 4.23 & 2.65 \\
\hline 8 & 2.18 & 3.10 & 1.72 & 1.98 & 3.03 & 1.52 \\
\hline 9 & 2.36 & 3.75 & 2.50 & 2.31 & 4.17 & 2.39 \\
\hline 10 & 1.45 & 1.86 & 1.36 & 1.92 & 2.64 & 1.82 \\
\hline 11 & 2.04 & 6.81 & 2.00 & 3.48 & 7.43 & 2.60 \\
\hline 12 & 2.54 & 5.99 & 2.44 & 2.32 & 5.52 & 1.77 \\
\hline 13 & 1.16 & -- & 1.29 & 1.80 & -- & 1.46 \\
\hline 14 & 2.72 & 4.04 & 2.56 & 3.01 & 5.73 & 2.88 \\
\hline 15 & 1.70 & 5.08 & 1.84 & -- & -- & -- \\
\hline 16 & 1.15 & 2.47 & 1.34 & 1.37 & 2.18 & 1.28 \\
\hline 17 & 3.91 & 5.13 & 3.23 & 3.52 & 6.69 & 2.74 \\
\hline 18 & 1.81 & 2.54 & 1.55 & 1.04 & 2.73 & 1.40 \\
\hline 19 & 2.49 & 4.72 & 3.41 & 2.07 & 4.21 & 2.23 \\
\hline 20 & 1.99 & 6.27 & 1.84 & -- & -- & -- \\
\hline 21 & 2.23 & 4.09 & 2.80 & 3.06 & 4.82 & 3.28 \\
\hline 22 & 2.61 & 3.35 & 2.25 & 2.12 & 2.27 & 1.57 \\
\hline 23 & 1.79 & 4.16 & 1.68 & 1.41 & 3.33 & 1.31 \\
\hline 24 & 1.73 & 3.57 & 1.80 & 2.08 & 4.10 & 1.94 \\
\hline 25 & 1.36 & 3.13 & 1.26 & 1.22 & -- & 1.21 \\
\hline 26 & 1.94 & 3.63 & 1.54 & 2.01 & 2.41 & 2.04 \\
\hline 27 & 2.25 & 6.00 & 1.93 & 1.75 & 6.91 & 1.89 \\
\hline 28 & 1.62 & 3.57 & 2.62 & 3.55 & 4.90 & 4.50 \\
\hline 29 & 2.44 & 3.82 & 2.39 & 3.09 & 3.81 & 3.25 \\
\hline 30 & 1.75 & 3.99 & 1.54 & 2.34 & 4.05 & 3.12 \\
\hline 31 & 0.96 & 1.30 & 1.28 & 1.22 & 1.48 & 1.28 \\
\hline 32 & 1.41 & 2.11 & 1.44 & 1.19 & 1.55 & 1.27 \\
\hline 33 & 3.12 & 3.22 & 3.32 & 3.48 & 5.06 & 3.32 \\
\hline 34 & 2.92 & 6.64 & 2.90 & 2.03 & 5.60 & 2.56 \\
\hline 35 & 1.02 & -- & 1.16 & 0.93 & -- & 1.05 \\
\hline 36 & 1.20 & -- & 1.45 & 3.03 & -- & 3.35 \\
\hline 37 & 1.98 & 5.45 & 1.83 & 3.67 & 7.55 & 2.91 \\
\hline 38 & 3.16 & 5.25 & 3.98 & 2.61 & 3.69 & 2.39 \\
\hline
\end{tabular}

Missing blood flow data are due to movement artifact or equipment failure during recording 
Table C.19. Raw data for 5 minute average forearm blood flow for prehypertensive group in study 2

\begin{tabular}{|c|c|c|c|c|c|c|}
\hline \multirow[b]{2}{*}{ Subject } & \multicolumn{3}{|c|}{ Pre supplementation } & \multicolumn{3}{|c|}{ Post supplementation } \\
\hline & Base & $\begin{array}{l}\text { Mental } \\
\text { Stress }\end{array}$ & Recovery & Base & $\begin{array}{l}\text { Mental } \\
\text { Stress }\end{array}$ & Recovery \\
\hline 39 & 2.49 & 4.68 & 2.53 & 3.34 & 5.31 & 3.24 \\
\hline 40 & 2.69 & 3.54 & 2.47 & 2.55 & -- & 2.24 \\
\hline 41 & 2.25 & 3.24 & 2.65 & 2.27 & 3.30 & 3.27 \\
\hline 42 & 4.21 & 6.91 & 3.96 & 3.46 & 5.57 & 3.17 \\
\hline 43 & 1.28 & 3.09 & 1.29 & -- & -- & -- \\
\hline 44 & 5.34 & 10.05 & 6.17 & 9.61 & 11.41 & 9.11 \\
\hline 45 & 3.72 & 8.37 & 3.66 & 3.08 & 7.01 & 3.28 \\
\hline 46 & 2.65 & 3.74 & 3.38 & 2.08 & 3.26 & 2.36 \\
\hline 47 & 2.40 & 5.48 & 2.85 & 4.09 & 4.66 & 3.50 \\
\hline 48 & 3.61 & 4.15 & 3.54 & 3.21 & 5.30 & 3.79 \\
\hline 49 & 4.38 & 7.50 & 4.00 & 3.48 & 4.13 & 2.79 \\
\hline 50 & 3.43 & 4.36 & 3.90 & 2.07 & 3.49 & 2.52 \\
\hline 51 & 5.93 & 5.69 & 5.79 & 3.49 & 2.91 & 3.58 \\
\hline 52 & 4.15 & 5.22 & 3.19 & 6.55 & 6.35 & 5.21 \\
\hline 53 & 2.23 & 6.06 & 2.09 & 2.52 & 3.71 & 1.37 \\
\hline 54 & 3.77 & 4.73 & 3.32 & 2.60 & 3.83 & 2.72 \\
\hline 55 & 2.52 & 2.65 & 2.35 & 2.19 & 2.61 & 2.20 \\
\hline 56 & 3.76 & 3.87 & 3.88 & 3.63 & 3.81 & 4.07 \\
\hline 57 & 4.03 & 8.85 & 3.38 & 3.61 & 8.61 & 3.48 \\
\hline 58 & 4.16 & 8.80 & 3.44 & 4.41 & 5.56 & 3.05 \\
\hline 59 & 2.81 & -- & 3.08 & 1.38 & -- & 1.69 \\
\hline 60 & 3.92 & 6.36 & 3.93 & 1.61 & 4.26 & 2.02 \\
\hline 61 & 2.35 & 3.96 & 2.38 & -- & -- & -- \\
\hline 62 & 2.59 & 5.23 & 2.67 & 5.91 & 8.77 & 5.84 \\
\hline 63 & 3.42 & 6.89 & 4.30 & 4.84 & 6.51 & 4.54 \\
\hline 64 & 3.81 & 5.17 & 3.43 & 4.25 & 4.57 & 2.95 \\
\hline 65 & 2.15 & 5.54 & 2.21 & 2.27 & 3.59 & 2.33 \\
\hline 66 & 6.40 & - & 6.17 & 2.81 & - & 2.45 \\
\hline 67 & 2.64 & 4.65 & 2.05 & 1.58 & -- & 1.58 \\
\hline
\end{tabular}

Missing blood flow data are due to movement artifact or equipment failure during recording 
Table C.20. Raw data for 5 minute average forearm vascular resistance for normotensive group in study 2

\begin{tabular}{|c|c|c|c|c|c|c|}
\hline \multirow[b]{2}{*}{ Subject } & \multicolumn{3}{|c|}{ Pre supplementation } & \multicolumn{3}{|c|}{ Post supplementation } \\
\hline & Base & $\begin{array}{l}\text { Mental } \\
\text { Stress }\end{array}$ & Recovery & Base & $\begin{array}{l}\text { Mental } \\
\text { Stress }\end{array}$ & Recovery \\
\hline 1 & 16.99 & 12.19 & 16.32 & 17.26 & 14.16 & 13.11 \\
\hline 2 & 27.35 & 25.61 & 25.75 & 35.37 & 26.01 & 34.85 \\
\hline 3 & 24.21 & 11.11 & 24.96 & 28.34 & 20.50 & 33.82 \\
\hline 4 & 28.98 & 12.93 & 31.64 & 19.88 & 7.94 & 25.62 \\
\hline 5 & 33.66 & 12.66 & 28.28 & 18.62 & 11.96 & 26.12 \\
\hline 6 & 33.22 & 29.51 & 36.66 & 22.46 & 30.71 & 30.75 \\
\hline 7 & 45.99 & 22.12 & 48.87 & 28.95 & 21.94 & 35.43 \\
\hline 8 & 42.23 & 32.29 & 48.65 & 45.92 & 36.93 & 60.39 \\
\hline 9 & 38.97 & 33.78 & 37.71 & 34.42 & 26.74 & 36.69 \\
\hline 10 & 67.21 & 59.29 & 66.82 & 46.16 & 36.49 & 52.20 \\
\hline 11 & 37.69 & 14.06 & 39.22 & 24.90 & 16.92 & 32.12 \\
\hline 12 & 37.93 & 19.73 & 40.66 & 37.83 & 19.51 & 52.49 \\
\hline 13 & 70.74 & -- & 68.13 & 43.69 & -- & 60.14 \\
\hline 14 & 37.29 & 28.47 & 39.62 & 28.74 & 18.46 & -- \\
\hline 15 & 49.64 & 18.70 & 44.07 & -- & -- & -- \\
\hline 16 & 76.10 & 44.11 & 70.75 & 63.97 & 45.05 & 70.44 \\
\hline 17 & 21.18 & 18.57 & 27.87 & 24.72 & 16.68 & 34.65 \\
\hline 18 & 55.04 & 46.71 & 67.59 & 80.54 & 37.58 & 59.70 \\
\hline 19 & 38.59 & 22.47 & 28.19 & 39.50 & 23.53 & 38.68 \\
\hline 20 & 44.76 & 20.22 & 50.66 & -- & -- & -- \\
\hline 21 & 40.18 & 23.50 & 31.57 & 30.95 & 21.07 & 28.57 \\
\hline 22 & 33.84 & 28.18 & 37.46 & 39.91 & 39.30 & 51.99 \\
\hline 23 & 48.97 & 25.98 & 56.82 & 60.20 & 29.39 & 71.06 \\
\hline 24 & 49.85 & 28.39 & 51.41 & 41.88 & 22.68 & 50.51 \\
\hline 25 & 66.44 & 31.94 & 77.24 & 73.03 & -- & 77.61 \\
\hline 26 & 46.85 & 31.43 & 62.82 & 40.55 & 40.56 & 41.73 \\
\hline 27 & 41.18 & 16.75 & 49.94 & 50.96 & 13.96 & 52.00 \\
\hline 28 & 55.22 & 26.59 & 35.73 & 22.82 & 18.09 & 19.19 \\
\hline 29 & 41.67 & 30.99 & 44.41 & 27.60 & 25.88 & 26.90 \\
\hline 30 & 47.10 & 26.13 & 53.49 & 33.59 & 24.23 & 28.09 \\
\hline 31 & 101.3 & 76.79 & 79.25 & 76.21 & 66.75 & 76.13 \\
\hline 32 & 66.52 & 47.95 & 67.41 & 81.65 & 68.59 & 79.19 \\
\hline 33 & 29.26 & 31.63 & 28.62 & 24.14 & 20.83 & 26.00 \\
\hline 34 & 30.98 & 16.72 & 33.73 & 45.46 & 18.65 & 38.25 \\
\hline 35 & 92.95 & -- & 84.29 & 83.08 & -- & 80.69 \\
\hline 36 & 84.72 & -- & 76.94 & 29.62 & -- & 27.71 \\
\hline 37 & 46.01 & 29.68 & 50.34 & 22.55 & 17.36 & 28.14 \\
\hline 38 & 27.07 & 18.67 & 24.83 & 30.16 & 24.45 & 35.57 \\
\hline
\end{tabular}

Missing blood flow data are due to movement artifact or equipment failure during recording 
Table C.21. Raw data for 5 minute average forearm vascular resistance for prehypertensive group in study 2

\begin{tabular}{|c|c|c|c|c|c|c|}
\hline \multirow[b]{2}{*}{ Subject } & \multicolumn{3}{|c|}{ Pre supplementation } & \multicolumn{3}{|c|}{ Post supplementation } \\
\hline & Base & $\begin{array}{c}\text { Mental } \\
\text { Stress }\end{array}$ & Recovery & Base & $\begin{array}{c}\text { Mental } \\
\text { Stress }\end{array}$ & Recovery \\
\hline 39 & 36.57 & 24.07 & 37.17 & 29.42 & 22.40 & 31.40 \\
\hline 40 & 37.92 & 34.36 & 43.98 & 38.66 & -- & 46.75 \\
\hline 41 & 44.86 & 36.57 & 38.45 & 38.68 & 33.66 & 26.86 \\
\hline 42 & 23.34 & 18.45 & 25.87 & 26.86 & 20.78 & 27.95 \\
\hline 43 & 73.32 & -- & 73.27 & -- & -- & -- \\
\hline 44 & 16.87 & 11.32 & 15.34 & 9.56 & 10.51 & 9.98 \\
\hline 45 & 24.49 & 15.15 & 26.01 & 26.22 & 16.09 & 26.68 \\
\hline 46 & 34.72 & 27.73 & 30.63 & 44.27 & 33.42 & 42.21 \\
\hline 47 & 35.11 & 18.09 & 30.41 & 20.48 & 20.87 & 23.90 \\
\hline 48 & 23.21 & 22.32 & 23.02 & 25.64 & 17.90 & 22.78 \\
\hline 49 & 18.51 & 12.92 & 20.60 & 25.10 & 24.69 & 31.64 \\
\hline 50 & 29.98 & 26.22 & 28.01 & 46.78 & 31.18 & 41.47 \\
\hline 51 & 14.47 & 17.72 & 15.44 & 22.40 & 31.91 & 26.64 \\
\hline 52 & 21.38 & 20.81 & 27.85 & 12.56 & 15.58 & 16.46 \\
\hline 53 & 51.64 & 25.84 & 59.20 & 48.41 & 34.64 & 79.67 \\
\hline 54 & 21.69 & 25.80 & 29.42 & 32.03 & 27.46 & 33.84 \\
\hline 55 & 36.14 & 39.17 & 38.59 & 38.44 & 34.49 & 38.64 \\
\hline 56 & 25.92 & 28.33 & 24.85 & 26.87 & 27.62 & 24.94 \\
\hline 57 & 26.20 & 15.26 & 32.13 & 26.57 & 14.41 & 29.01 \\
\hline 58 & 23.08 & 12.92 & 28.75 & 22.56 & 22.66 & 34.14 \\
\hline 59 & 43.04 & -- & 39.12 & 83.39 & -- & 70.34 \\
\hline 60 & 23.48 & 16.72 & 24.02 & 58.51 & 25.34 & 51.22 \\
\hline 61 & 43.93 & 32.30 & 44.13 & -- & -- & -- \\
\hline 62 & 37.23 & 20.77 & 36.85 & 18.74 & 14.50 & 20.19 \\
\hline 63 & 29.58 & 16.35 & 23.69 & 16.99 & 14.70 & 18.58 \\
\hline 64 & 25.94 & 27.69 & 30.33 & 22.79 & 28.92 & 36.33 \\
\hline 65 & 44.02 & 23.48 & 46.28 & 45.32 & 35.84 & 46.45 \\
\hline 66 & 15.40 & -- & 17.34 & 36.66 & -- & 44.11 \\
\hline 67 & 44.32 & 31.42 & 57.95 & 56.63 & -- & 67.97 \\
\hline
\end{tabular}

Missing blood flow data are due to movement artifact or equipment failure during recording 
Table C.22. Raw data for 5 minute average forearm vascular conductance for normotensive group in study 2

\begin{tabular}{|c|c|c|c|c|c|c|}
\hline \multirow[b]{2}{*}{ Subject } & \multicolumn{3}{|c|}{ Pre supplementation } & \multicolumn{3}{|c|}{ Post supplementation } \\
\hline & Base & $\begin{array}{c}\text { Mental } \\
\text { Stress }\end{array}$ & Recovery & Base & $\begin{array}{c}\text { Mental } \\
\text { Stress }\end{array}$ & Recovery \\
\hline 1 & 0.060 & 0.084 & 0.062 & 0.058 & 0.072 & 0.077 \\
\hline 2 & 0.037 & 0.041 & 0.039 & 0.028 & 0.040 & 0.029 \\
\hline 3 & 0.042 & 0.096 & 0.041 & 0.040 & 0.059 & 0.031 \\
\hline 4 & 0.035 & 0.078 & 0.032 & 0.052 & 0.130 & 0.039 \\
\hline 5 & 0.030 & 0.084 & 0.037 & 0.057 & 0.087 & 0.039 \\
\hline 6 & 0.030 & 0.035 & 0.027 & 0.045 & 0.033 & 0.033 \\
\hline 7 & 0.023 & 0.046 & 0.021 & 0.036 & 0.046 & 0.029 \\
\hline 8 & 0.024 & 0.032 & 0.021 & 0.022 & 0.029 & 0.017 \\
\hline 9 & 0.026 & 0.037 & 0.027 & 0.029 & 0.041 & 0.027 \\
\hline 10 & 0.015 & 0.017 & 0.015 & 0.022 & 0.028 & 0.019 \\
\hline 11 & 0.027 & 0.073 & 0.026 & 0.040 & 0.072 & 0.032 \\
\hline 12 & 0.026 & 0.052 & 0.025 & 0.027 & 0.052 & 0.019 \\
\hline 13 & 0.014 & -- & 0.015 & 0.023 & -- & 0.017 \\
\hline 14 & 0.027 & 0.038 & 0.025 & 0.035 & 0.055 & -- \\
\hline 15 & 0.021 & 0.056 & 0.023 & -- & -- & -- \\
\hline 16 & 0.013 & 0.024 & 0.014 & 0.016 & 0.022 & 0.014 \\
\hline 17 & 0.048 & 0.054 & 0.036 & 0.042 & 0.064 & 0.029 \\
\hline 18 & 0.018 & 0.022 & 0.015 & 0.012 & 0.029 & 0.017 \\
\hline 19 & 0.026 & 0.045 & 0.036 & 0.025 & 0.045 & 0.026 \\
\hline 20 & 0.022 & 0.064 & 0.020 & -- & -- & -- \\
\hline 21 & 0.025 & 0.043 & 0.032 & 0.033 & 0.051 & 0.036 \\
\hline 22 & 0.030 & 0.036 & 0.027 & 0.026 & 0.027 & 0.021 \\
\hline 23 & 0.020 & 0.042 & 0.018 & 0.017 & 0.039 & 0.015 \\
\hline 24 & 0.020 & 0.039 & 0.020 & 0.025 & 0.045 & 0.020 \\
\hline 25 & 0.015 & 0.031 & 0.013 & 0.014 & -- & 0.013 \\
\hline 26 & 0.022 & 0.035 & 0.016 & 0.025 & 0.026 & 0.024 \\
\hline 27 & 0.025 & 0.063 & 0.021 & 0.021 & 0.073 & 0.020 \\
\hline 28 & 0.018 & 0.038 & 0.029 & 0.044 & 0.055 & 0.053 \\
\hline 29 & 0.024 & 0.032 & 0.023 & 0.036 & 0.039 & 0.037 \\
\hline 30 & 0.021 & 0.040 & 0.019 & 0.030 & 0.044 & 0.037 \\
\hline 31 & 0.010 & 0.013 & 0.013 & 0.013 & 0.015 & 0.014 \\
\hline 32 & 0.015 & 0.021 & 0.015 & 0.012 & 0.015 & 0.013 \\
\hline 33 & 0.034 & 0.033 & 0.036 & 0.041 & 0.054 & 0.039 \\
\hline 34 & 0.032 & 0.064 & 0.030 & 0.023 & 0.055 & 0.026 \\
\hline 35 & 0.011 & -- & 0.012 & 0.012 & -- & 0.012 \\
\hline 36 & 0.012 & -- & 0.013 & 0.034 & -- & 0.036 \\
\hline 37 & 0.022 & 0.052 & 0.020 & 0.045 & 0.082 & 0.036 \\
\hline 38 & 0.038 & 0.055 & 0.041 & 0.033 & 0.042 & 0.028 \\
\hline
\end{tabular}

Missing blood flow data are due to movement artifact or equipment failure during recording 
Table C.23. Raw data for 5 minute average forearm vascular conductance for prehypertensive group in study 2

\begin{tabular}{|c|c|c|c|c|c|c|}
\hline \multirow[b]{2}{*}{ Subject } & \multicolumn{3}{|c|}{ Pre supplementation } & \multicolumn{3}{|c|}{ Post supplementation } \\
\hline & Base & $\begin{array}{l}\text { Mental } \\
\text { Stress }\end{array}$ & Recovery & Base & $\begin{array}{l}\text { Mental } \\
\text { Stress }\end{array}$ & Recovery \\
\hline 39 & 0.028 & 0.047 & 0.027 & 0.034 & 0.051 & 0.032 \\
\hline 40 & 0.028 & 0.032 & 0.025 & 0.027 & -- & 0.022 \\
\hline 41 & 0.022 & 0.029 & 0.026 & 0.026 & 0.036 & 0.038 \\
\hline 42 & 0.043 & 0.061 & 0.039 & 0.038 & 0.053 & 0.036 \\
\hline 43 & 0.014 & -- & 0.014 & -- & -- & -- \\
\hline 44 & 0.059 & 0.089 & 0.066 & 0.105 & 0.096 & 0.101 \\
\hline 45 & 0.041 & 0.076 & 0.039 & 0.038 & 0.066 & 0.038 \\
\hline 46 & 0.029 & 0.037 & 0.036 & 0.023 & 0.033 & 0.025 \\
\hline 47 & 0.029 & 0.055 & 0.033 & 0.049 & 0.052 & 0.043 \\
\hline 48 & 0.045 & 0.045 & 0.044 & 0.039 & 0.057 & 0.044 \\
\hline 49 & 0.054 & 0.082 & 0.049 & 0.040 & 0.043 & 0.032 \\
\hline 50 & 0.034 & 0.039 & 0.036 & 0.022 & 0.033 & 0.025 \\
\hline 51 & 0.069 & 0.057 & 0.065 & 0.045 & 0.032 & 0.039 \\
\hline 52 & 0.047 & 0.052 & 0.036 & 0.080 & 0.066 & 0.061 \\
\hline 53 & 0.019 & 0.042 & 0.018 & 0.025 & 0.030 & 0.013 \\
\hline 54 & 0.047 & 0.048 & 0.035 & 0.031 & 0.040 & 0.030 \\
\hline 55 & 0.028 & 0.026 & 0.026 & 0.026 & 0.030 & 0.026 \\
\hline 56 & 0.039 & 0.037 & 0.041 & 0.037 & 0.037 & 0.040 \\
\hline 57 & 0.039 & 0.078 & 0.032 & 0.038 & 0.082 & 0.035 \\
\hline 58 & 0.044 & 0.088 & 0.035 & 0.045 & 0.054 & 0.030 \\
\hline 59 & 0.023 & -- & 0.026 & 0.013 & -- & 0.015 \\
\hline 60 & 0.043 & 0.062 & 0.042 & 0.017 & 0.042 & 0.021 \\
\hline 61 & 0.023 & 0.036 & 0.023 & -- & -- & -- \\
\hline 62 & 0.028 & 0.049 & 0.027 & 0.054 & 0.070 & 0.050 \\
\hline 63 & 0.034 & 0.061 & 0.043 & 0.059 & 0.069 & 0.055 \\
\hline 64 & 0.039 & 0.041 & 0.033 & 0.045 & 0.039 & 0.028 \\
\hline 65 & 0.023 & 0.051 & 0.022 & 0.022 & 0.032 & 0.022 \\
\hline 66 & 0.065 & -- & 0.058 & 0.028 & -- & 0.024 \\
\hline 67 & 0.024 & 0.038 & 0.017 & 0.018 & -- & 0.015 \\
\hline
\end{tabular}

Missing blood flow data are due to movement artifact or equipment failure during recording 
Table C.24. Raw data for 5 minute average calf blood flow for normotensive group in study 2

\begin{tabular}{|c|c|c|c|c|c|c|}
\hline \multirow[b]{2}{*}{ Subject } & \multicolumn{3}{|c|}{ Pre supplementation } & \multicolumn{3}{|c|}{ Post supplementation } \\
\hline & Base & $\begin{array}{l}\text { Mental } \\
\text { Stress }\end{array}$ & Recovery & Base & $\begin{array}{l}\text { Mental } \\
\text { Stress }\end{array}$ & Recovery \\
\hline 1 & 3.18 & 4.34 & -- & 2.59 & 3.15 & 2.89 \\
\hline 2 & 1.74 & 2.03 & 1.98 & 2.16 & 2.32 & 2.21 \\
\hline 3 & 3.58 & 4.50 & 3.50 & 2.21 & 2.79 & 1.89 \\
\hline 4 & 3.94 & 7.66 & 4.59 & 3.29 & 6.48 & 2.71 \\
\hline 5 & 2.18 & 4.71 & 2.00 & 3.14 & 4.25 & 2.47 \\
\hline 6 & 3.91 & 3.76 & 3.53 & 3.51 & 3.73 & 3.67 \\
\hline 7 & 2.81 & 3.62 & 2.59 & 2.12 & 2.00 & 1.55 \\
\hline 8 & 1.75 & 2.36 & 1.65 & 2.04 & 2.92 & 2.08 \\
\hline 9 & 1.70 & 2.74 & 1.87 & 1.89 & 2.57 & 1.79 \\
\hline 10 & 1.57 & 1.85 & 1.33 & -- & -- & -- \\
\hline 11 & 1.21 & 1.20 & 1.12 & 2.26 & 2.37 & 2.22 \\
\hline 12 & 1.53 & 2.79 & 1.35 & 3.39 & 4.61 & 3.19 \\
\hline 13 & 0.98 & 1.04 & 1.00 & 1.60 & 1.59 & 1.51 \\
\hline 14 & 2.25 & 3.99 & 2.36 & 2.54 & 4.22 & 2.51 \\
\hline 15 & 0.93 & 1.90 & 0.87 & -- & -- & -- \\
\hline 16 & 1.43 & 2.34 & 1.66 & 1.72 & 2.06 & 1.55 \\
\hline 17 & 2.14 & 3.06 & 1.92 & 3.44 & 5.55 & 3.18 \\
\hline 18 & 1.26 & 1.83 & 1.23 & 0.95 & 1.44 & 0.96 \\
\hline 19 & 2.49 & 2.81 & 2.89 & 2.70 & 2.70 & 2.89 \\
\hline 20 & 1.79 & 1.98 & 2.12 & -- & -- & -- \\
\hline 21 & 1.94 & 2.37 & 2.07 & 1.87 & 2.12 & 1.91 \\
\hline 22 & 1.52 & 1.32 & 1.58 & 1.79 & 1.79 & 1.80 \\
\hline 23 & 1.66 & 3.83 & 1.73 & 1.37 & 3.25 & 1.47 \\
\hline 24 & 1.84 & 3.10 & 1.92 & 2.00 & 2.69 & 1.91 \\
\hline 25 & 1.51 & 1.93 & 1.29 & -- & -- & -- \\
\hline 26 & 1.40 & 2.44 & 1.23 & -- & -- & -- \\
\hline 27 & -- & -- & -- & -- & -- & -- \\
\hline 28 & 3.02 & 3.46 & 2.26 & 2.14 & 2.24 & 2.03 \\
\hline 29 & 1.80 & 2.41 & 1.76 & 1.50 & 2.22 & 1.58 \\
\hline 30 & 2.38 & 2.77 & 1.42 & 2.01 & 3.00 & 1.96 \\
\hline 31 & 0.96 & 1.30 & 1.28 & 1.62 & 1.53 & 1.69 \\
\hline 32 & 1.50 & 1.72 & 1.41 & 1.60 & 1.58 & 1.36 \\
\hline 33 & 1.60 & 1.72 & 1.56 & 3.01 & 3.37 & 2.59 \\
\hline 34 & 1.70 & 4.11 & 2.32 & 1.60 & 2.98 & 1.98 \\
\hline 35 & 1.94 & -- & 1.84 & 2.42 & 2.27 & 2.47 \\
\hline 36 & 1.67 & 1.53 & 1.57 & 1.71 & 1.53 & 1.62 \\
\hline 37 & 2.08 & 1.80 & 1.50 & 2.45 & 2.40 & 2.21 \\
\hline 38 & 2.65 & 2.79 & 2.62 & 4.01 & 3.39 & 4.15 \\
\hline
\end{tabular}

Missing blood flow data are due to movement artifact or equipment failure during recording 
Table C.25. Raw data for 5 minute average calf blood flow for prehypertensive group in study 2

\begin{tabular}{|c|c|c|c|c|c|c|}
\hline \multirow[b]{2}{*}{ Subject } & \multicolumn{3}{|c|}{ Pre supplementation } & \multicolumn{3}{|c|}{ Post supplementation } \\
\hline & Base & $\begin{array}{l}\text { Mental } \\
\text { Stress }\end{array}$ & Recovery & Base & $\begin{array}{l}\text { Mental } \\
\text { Stress }\end{array}$ & Recovery \\
\hline 39 & 2.78 & 4.01 & 2.87 & 3.42 & 4.11 & 3.57 \\
\hline 40 & 2.41 & 2.19 & 2.06 & 2.69 & 2.98 & 2.74 \\
\hline 41 & 2.02 & 2.74 & 2.04 & 1.80 & 2.35 & 1.84 \\
\hline 42 & 3.21 & 5.34 & 3.27 & 4.80 & 5.18 & 3.61 \\
\hline 43 & -- & -- & -- & -- & -- & -- \\
\hline 44 & 2.20 & 3.78 & 2.41 & 3.57 & 5.79 & 3.86 \\
\hline 45 & 3.88 & 5.80 & 3.93 & 3.93 & 5.64 & 4.63 \\
\hline 46 & 1.84 & 2.28 & 2.28 & 1.66 & 2.20 & 1.70 \\
\hline 47 & 3.04 & 3.51 & 3.38 & 4.43 & 3.40 & 3.45 \\
\hline 48 & 1.64 & 2.08 & 1.65 & -- & -- & -- \\
\hline 49 & -- & -- & -- & 2.84 & 3.29 & 2.87 \\
\hline 50 & 1.74 & 2.91 & 1.51 & 1.36 & 1.59 & 1.37 \\
\hline 51 & 2.05 & 2.61 & 1.90 & 2.60 & 2.88 & 3.11 \\
\hline 52 & 3.60 & 3.50 & 3.11 & 3.51 & 3.84 & 2.86 \\
\hline 53 & 3.22 & 4.84 & 2.89 & 4.26 & 5.06 & 2.94 \\
\hline 54 & 2.42 & 2.32 & 2.36 & 3.52 & 3.89 & 3.92 \\
\hline 55 & 2.03 & 2.26 & 2.02 & 1.89 & 2.38 & 1.91 \\
\hline 56 & 3.17 & 3.67 & 3.23 & 4.12 & 4.15 & 4.31 \\
\hline 57 & 3.88 & 6.40 & 3.39 & 2.76 & 5.02 & 2.70 \\
\hline 58 & 3.86 & 2.47 & 2.49 & 1.76 & 1.46 & 1.29 \\
\hline 59 & 2.73 & 4.58 & 3.03 & 2.01 & -- & 2.09 \\
\hline 60 & 2.00 & 2.80 & 1.91 & 1.69 & 2.13 & 1.93 \\
\hline 61 & 1.62 & 2.46 & 1.50 & -- & -- & -- \\
\hline 62 & 1.76 & 1.73 & 1.46 & 1.53 & 2.16 & 1.51 \\
\hline 63 & 2.42 & 3.10 & 2.28 & 2.71 & 3.49 & 3.33 \\
\hline 64 & 2.68 & 2.94 & 2.30 & 3.82 & 3.25 & 3.09 \\
\hline 65 & 2.09 & 2.61 & 3.34 & 2.19 & 2.57 & 2.25 \\
\hline 66 & 2.28 & -- & 2.43 & 2.23 & -- & 2.04 \\
\hline 67 & 3.04 & 3.93 & 4.28 & 1.56 & 2.16 & 1.54 \\
\hline
\end{tabular}

Missing blood flow data are due to movement artifact or equipment failure during recording 
Table C.26. Raw data for 5 minute average calf vascular resistance for normotensive group in study 2

\begin{tabular}{|c|c|c|c|c|c|c|}
\hline \multirow[b]{2}{*}{ Subject } & \multicolumn{3}{|c|}{ Pre supplementation } & \multicolumn{3}{|c|}{ Post supplementation } \\
\hline & Base & $\begin{array}{l}\text { Mental } \\
\text { Stress }\end{array}$ & Recovery & Base & $\begin{array}{c}\text { Mental } \\
\text { Stress }\end{array}$ & Recovery \\
\hline 1 & 31.03 & 32.37 & -- & 34.72 & 34.50 & 34.12 \\
\hline 2 & 52.15 & 52.32 & 46.31 & 45.16 & 49.29 & 45.16 \\
\hline 3 & 27.66 & 26.09 & 30.37 & 45.53 & 43.42 & 50.33 \\
\hline 4 & 25.97 & 15.63 & 22.99 & 30.60 & 19.83 & 37.16 \\
\hline 5 & 43.80 & 26.03 & 44.69 & 33.23 & 27.30 & 47.28 \\
\hline 6 & 25.62 & 27.67 & 27.99 & 23.18 & 23.85 & 22.87 \\
\hline 7 & 33.03 & 27.82 & 38.70 & 43.67 & 47.65 & 61.59 \\
\hline 8 & 50.95 & 42.00 & 50.41 & 44.52 & 37.16 & 43.35 \\
\hline 9 & 54.15 & 44.34 & 50.76 & 42.40 & 40.61 & 48.78 \\
\hline 10 & 62.13 & 60.09 & 68.11 & -- & -- & -- \\
\hline 11 & 63.18 & 82.52 & 70.01 & 38.46 & 47.92 & 37.19 \\
\hline 12 & 62.83 & 42.31 & 74.26 & 25.87 & 23.10 & 29.04 \\
\hline 13 & 83.32 & 99.40 & 88.95 & 49.52 & 61.58 & 57.09 \\
\hline 14 & 44.99 & 29.51 & 45.17 & 34.29 & 25.52 & -- \\
\hline 15 & 93.09 & 54.98 & 94.13 & -- & -- & -- \\
\hline 16 & 61.02 & 46.00 & 57.15 & 50.72 & 48.15 & 58.50 \\
\hline 17 & 38.43 & 33.04 & 47.44 & 24.89 & 18.79 & 29.65 \\
\hline 18 & 78.96 & 63.94 & 87.09 & 88.47 & 67.29 & 89.65 \\
\hline 19 & 38.37 & 38.26 & 33.28 & 30.27 & 34.80 & 29.64 \\
\hline 20 & 49.94 & 51.17 & 43.25 & -- & -- & -- \\
\hline 21 & 46.00 & 41.07 & 41.78 & 49.82 & 44.49 & 48.70 \\
\hline 22 & 58.77 & 72.71 & 56.56 & 46.09 & 47.67 & 42.22 \\
\hline 23 & 53.14 & 30.72 & 55.10 & 63.15 & 28.22 & 60.96 \\
\hline 24 & 46.66 & 32.05 & 47.79 & 42.39 & 34.43 & 50.66 \\
\hline 25 & 59.57 & 52.17 & 75.16 & -- & -- & -- \\
\hline 26 & 65.85 & 43.86 & 78.13 & -- & -- & -- \\
\hline 27 & -- & -- & -- & -- & -- & -- \\
\hline 28 & 29.54 & 27.59 & 41.58 & 37.55 & 40.40 & 41.80 \\
\hline 29 & 56.41 & 48.90 & 61.41 & 56.85 & 43.87 & 55.94 \\
\hline 30 & 34.71 & 36.27 & 58.14 & 38.69 & 31.49 & 42.68 \\
\hline 31 & 101.3 & 73.15 & 79.25 & 56.94 & 64.48 & 57.25 \\
\hline 32 & 64.73 & 58.56 & 68.61 & 61.28 & 67.34 & 73.66 \\
\hline 33 & 57.38 & 57.52 & 59.33 & 27.94 & 28.34 & 33.13 \\
\hline 34 & 54.10 & 28.00 & 42.52 & 57.12 & 36.60 & 49.68 \\
\hline 35 & 47.81 & -- & 54.44 & 31.51 & 38.10 & 34.06 \\
\hline 36 & 61.15 & 77.61 & 70.68 & 52.42 & 65.43 & 57.35 \\
\hline 37 & 43.44 & 61.54 & 62.21 & 33.24 & 40.01 & 37.50 \\
\hline 38 & 31.89 & 35.47 & 37.03 & 19.48 & 26.01 & 20.76 \\
\hline
\end{tabular}

Missing blood flow data are due to movement artifact or equipment failure during recording 
Table C.27. Raw data 5 minute average for calf vascular resistance for prehypertensive group in study 2

\begin{tabular}{|c|c|c|c|c|c|c|}
\hline \multirow[b]{2}{*}{ Subject } & \multicolumn{3}{|c|}{ Pre supplementation } & \multicolumn{3}{|c|}{ Post supplementation } \\
\hline & Base & $\begin{array}{l}\text { Mental } \\
\text { Stress }\end{array}$ & Recovery & Base & $\begin{array}{l}\text { Mental } \\
\text { Stress }\end{array}$ & Recovery \\
\hline 39 & 32.54 & 28.64 & 32.48 & 28.65 & 25.89 & 28.30 \\
\hline 40 & 41.44 & 52.62 & 49.10 & 35.87 & 38.69 & 37.58 \\
\hline 41 & 49.91 & 42.67 & 49.57 & 47.92 & 40.91 & 46.85 \\
\hline 42 & 30.77 & 21.98 & 31.12 & 18.93 & 21.94 & 24.77 \\
\hline 43 & -- & -- & -- & -- & -- & -- \\
\hline 44 & 41.22 & 33.78 & 39.25 & 25.82 & 21.97 & 23.74 \\
\hline 45 & 23.27 & 21.87 & 23.85 & 20.58 & 19.81 & 18.51 \\
\hline 46 & 49.26 & 45.69 & 41.68 & 55.08 & 45.17 & 56.74 \\
\hline 47 & 27.68 & 32.66 & 25.62 & 18.95 & 26.52 & 23.41 \\
\hline 48 & 49.30 & 43.84 & 49.50 & -- & -- & -- \\
\hline 49 & -- & -- & - & 30.86 & 30.26 & 30.73 \\
\hline 50 & 58.92 & 46.26 & 71.28 & 69.95 & 67.33 & 73.96 \\
\hline 51 & 41.84 & 39.78 & 47.12 & 30.06 & 31.36 & 29.72 \\
\hline 52 & 24.72 & 31.31 & 28.62 & 23.56 & 25.21 & 29.92 \\
\hline 53 & 35.64 & 31.86 & 41.65 & 24.69 & 26.16 & 36.61 \\
\hline 54 & 33.86 & 45.34 & 41.02 & 23.80 & 24.90 & 23.14 \\
\hline 55 & 44.70 & 46.05 & 45.36 & 43.97 & 37.36 & 44.24 \\
\hline 56 & 30.71 & 29.08 & 29.55 & 23.67 & 24.66 & 23.45 \\
\hline 57 & 26.98 & 19.43 & 31.75 & 34.72 & 22.79 & 37.72 \\
\hline 58 & 25.22 & 46.19 & 39.48 & 57.89 & 73.91 & 81.72 \\
\hline 59 & 44.67 & 34.02 & 39.46 & 54.34 & -- & 56.40 \\
\hline 60 & 46.41 & 38.95 & 49.71 & 55.21 & 49.03 & 49.36 \\
\hline 61 & 63.84 & 50.32 & 69.44 & -- & -- & -- \\
\hline 62 & 54.35 & 62.30 & 68.44 & 71.91 & 58.02 & 78.05 \\
\hline 63 & 41.81 & 36.48 & 44.80 & 30.38 & 27.19 & 25.08 \\
\hline 64 & 37.40 & 47.50 & 45.54 & 25.11 & 39.20 & 35.25 \\
\hline 65 & 45.78 & 42.50 & 33.21 & 46.77 & 45.28 & 48.40 \\
\hline 66 & 43.04 & -- & 44.28 & 45.41 & -- & 52.29 \\
\hline 67 & 36.64 & 33.86 & 27.76 & 57.25 & 54.62 & 68.36 \\
\hline
\end{tabular}

Missing blood flow data are due to movement artifact or equipment failure during recording 
Table C.28. Raw data for 5 minute average calf vascular conductance for normotensive group in study 2

\begin{tabular}{|c|c|c|c|c|c|c|}
\hline \multirow[b]{2}{*}{ Subject } & \multicolumn{3}{|c|}{ Pre supplementation } & \multicolumn{3}{|c|}{ Post supplementation } \\
\hline & Base & $\begin{array}{l}\text { Mental } \\
\text { Stress }\end{array}$ & Recovery & Base & $\begin{array}{c}\text { Mental } \\
\text { Stress }\end{array}$ & Recovery \\
\hline 1 & 0.033 & 0.037 & -- & 0.029 & 0.030 & 0.030 \\
\hline 2 & 0.019 & 0.020 & 0.022 & 0.022 & 0.020 & 0.022 \\
\hline 3 & 0.036 & 0.041 & 0.035 & 0.023 & 0.025 & 0.020 \\
\hline 4 & 0.039 & 0.065 & 0.044 & 0.033 & 0.057 & 0.027 \\
\hline 5 & 0.023 & 0.048 & 0.023 & 0.031 & 0.041 & 0.022 \\
\hline 6 & 0.040 & 0.036 & 0.036 & 0.043 & 0.042 & 0.044 \\
\hline 7 & 0.031 & 0.037 & 0.027 & 0.024 & 0.022 & 0.017 \\
\hline 8 & 0.020 & 0.024 & 0.020 & 0.022 & 0.028 & 0.023 \\
\hline 9 & 0.019 & 0.027 & 0.020 & 0.024 & 0.025 & 0.021 \\
\hline 10 & 0.016 & 0.017 & 0.015 & -- & -- & -- \\
\hline 11 & 0.016 & 0.013 & 0.014 & 0.026 & 0.023 & 0.027 \\
\hline 12 & 0.016 & 0.024 & 0.014 & 0.039 & 0.044 & 0.035 \\
\hline 13 & 0.012 & 0.011 & 0.011 & 0.020 & 0.017 & 0.018 \\
\hline 14 & 0.022 & 0.038 & 0.024 & 0.029 & 0.041 & -- \\
\hline 15 & 0.011 & 0.021 & 0.011 & -- & -- & -- \\
\hline 16 & 0.016 & 0.023 & 0.018 & 0.020 & 0.021 & 0.017 \\
\hline 17 & 0.026 & 0.033 & 0.022 & 0.041 & 0.054 & 0.034 \\
\hline 18 & 0.013 & 0.016 & 0.012 & 0.011 & 0.015 & 0.012 \\
\hline 19 & 0.026 & 0.027 & 0.030 & 0.033 & 0.029 & 0.034 \\
\hline 20 & 0.020 & 0.020 & 0.023 & -- & -- & -- \\
\hline 21 & 0.022 & 0.025 & 0.024 & 0.020 & 0.023 & 0.021 \\
\hline 22 & 0.017 & 0.014 & 0.019 & 0.022 & 0.021 & 0.024 \\
\hline 23 & 0.019 & 0.039 & 0.018 & 0.016 & 0.037 & 0.017 \\
\hline 24 & 0.021 & 0.034 & 0.021 & 0.024 & 0.030 & 0.020 \\
\hline 25 & 0.017 & 0.019 & 0.013 & -- & -- & -- \\
\hline 26 & 0.016 & 0.024 & 0.013 & -- & -- & -- \\
\hline 27 & - & -- & - & -- & -- & -- \\
\hline 28 & 0.034 & 0.036 & 0.025 & 0.027 & 0.025 & 0.024 \\
\hline 29 & 0.018 & 0.021 & 0.017 & 0.018 & 0.023 & 0.018 \\
\hline 30 & 0.029 & 0.028 & 0.018 & 0.026 & 0.032 & 0.023 \\
\hline 31 & 0.010 & 0.014 & 0.013 & 0.018 & 0.016 & 0.018 \\
\hline 32 & 0.015 & 0.017 & 0.015 & 0.016 & 0.015 & 0.014 \\
\hline 33 & 0.018 & 0.017 & 0.017 & 0.036 & 0.035 & 0.030 \\
\hline 34 & 0.019 & 0.040 & 0.024 & 0.018 & 0.029 & 0.020 \\
\hline 35 & 0.021 & -- & 0.019 & 0.032 & 0.026 & 0.029 \\
\hline 36 & 0.016 & 0.013 & 0.014 & 0.019 & 0.015 & 0.017 \\
\hline 37 & 0.023 & 0.017 & 0.017 & 0.030 & 0.026 & 0.027 \\
\hline 38 & 0.031 & 0.029 & 0.027 & 0.051 & 0.039 & 0.049 \\
\hline
\end{tabular}

Missing blood flow data are due to movement artifact or equipment failure during recording 
Table C.29. Raw data for 5 minute average calf vascular conductance for prehypertensive group in study 2

\begin{tabular}{|c|c|c|c|c|c|c|}
\hline \multirow[b]{2}{*}{ Subject } & \multicolumn{3}{|c|}{ Pre supplementation } & \multicolumn{3}{|c|}{ Post supplementation } \\
\hline & Base & $\begin{array}{l}\text { Mental } \\
\text { Stress }\end{array}$ & Recovery & Base & $\begin{array}{l}\text { Mental } \\
\text { Stress }\end{array}$ & Recovery \\
\hline 39 & 0.031 & 0.040 & 0.031 & 0.035 & 0.039 & 0.035 \\
\hline 40 & 0.025 & 0.020 & 0.021 & 0.028 & 0.027 & 0.027 \\
\hline 41 & 0.020 & 0.024 & 0.020 & 0.021 & 0.025 & 0.021 \\
\hline 42 & 0.033 & 0.046 & 0.032 & 0.053 & 0.049 & 0.041 \\
\hline 43 & -- & -- & -- & -- & -- & -- \\
\hline 44 & 0.024 & 0.033 & 0.026 & 0.039 & 0.049 & 0.043 \\
\hline 45 & 0.043 & 0.053 & 0.042 & 0.049 & 0.054 & 0.054 \\
\hline 46 & 0.020 & 0.022 & 0.024 & 0.018 & 0.023 & 0.018 \\
\hline 47 & 0.036 & 0.031 & 0.039 & 0.054 & 0.038 & 0.043 \\
\hline 48 & 0.021 & 0.023 & 0.020 & -- & -- & -- \\
\hline 49 & -- & -- & -- & 0.032 & 0.035 & 0.033 \\
\hline 50 & 0.017 & 0.026 & 0.014 & 0.014 & 0.015 & 0.014 \\
\hline 51 & 0.024 & 0.025 & 0.021 & 0.033 & 0.032 & 0.034 \\
\hline 52 & 0.041 & 0.035 & 0.035 & 0.043 & 0.040 & 0.034 \\
\hline 53 & 0.028 & 0.035 & 0.024 & 0.042 & 0.042 & 0.027 \\
\hline 54 & 0.030 & 0.023 & 0.025 & 0.043 & 0.041 & 0.043 \\
\hline 55 & 0.022 & 0.022 & 0.022 & 0.023 & 0.027 & 0.023 \\
\hline 56 & 0.033 & 0.035 & 0.034 & 0.042 & 0.041 & 0.043 \\
\hline 57 & 0.037 & 0.056 & 0.032 & 0.029 & 0.048 & 0.027 \\
\hline 58 & 0.041 & 0.024 & 0.025 & 0.018 & 0.014 & 0.012 \\
\hline 59 & 0.023 & 0.031 & 0.025 & 0.018 & -- & 0.018 \\
\hline 60 & 0.022 & 0.027 & 0.020 & 0.018 & 0.021 & 0.020 \\
\hline 61 & 0.016 & 0.022 & 0.015 & -- & -- & -- \\
\hline 62 & 0.019 & 0.016 & 0.015 & 0.014 & 0.017 & 0.013 \\
\hline 63 & 0.024 & 0.028 & 0.023 & 0.033 & 0.037 & 0.040 \\
\hline 64 & 0.028 & 0.023 & 0.022 & 0.040 & 0.028 & 0.029 \\
\hline 65 & 0.022 & 0.024 & 0.033 & 0.021 & 0.022 & 0.021 \\
\hline 66 & 0.023 & -- & 0.023 & 0.022 & -- & 0.020 \\
\hline 67 & 0.027 & 0.032 & 0.036 & 0.018 & 0.020 & 0.015 \\
\hline
\end{tabular}

Missing blood flow data are due to movement artifact or equipment failure during recording 


\section{Appendix D}

Appendix D-1. Repeated Measures ANOVA in normotensive group, 2 Time (pre/post) vs. 2 Drug (Fish Oil/Placebo) resting values presented; two-tailed significance

\begin{tabular}{lccc}
\multicolumn{1}{c}{ Variable } & Time & $\begin{array}{c}\text { Time } \mathbf{x} \text { Drug } \\
\text { Interaction }\end{array}$ & $\begin{array}{c}\mathbf{N} \text { (fish } \\
\text { oil/placebo) }\end{array}$ \\
\hline SAP $(\mathrm{mmHg})$ & .194 & .169 & $19 / 19$ \\
DAP (mmHg) & .279 & .693 & $19 / 19$ \\
MAP (mmHg) & .778 & .420 & $19 / 19$ \\
HR (beats/min) & .464 & .427 & $19 / 19$ \\
MSNA (bursts/min) & .729 & .160 & $16 / 17$ \\
MSNA (bursts/100hb) & .864 & .340 & $16 / 17$ \\
FBF (mL/100mL/min) & .045 & .814 & $18 / 18$ \\
FVR (mmHg/mL/100mL/min) & .020 & .681 & $18 / 18$ \\
FVC (mL/100mL/min/mmHg) & .005 & .947 & $18 / 18$ \\
CBF (mL/100mL/min) & .138 & .701 & $16 / 16$ \\
CVR (mmHg/mL/100mL/min) & .006 & .555 & $16 / 16$ \\
CVC (mL/100mL/min/mmHg) & .012 & .926 & $16 / 16$
\end{tabular}

Appendix D-2. Repeated Measures ANOVA in prehypertensive group, 2 Time (pre/post) vs. 2 Drug (Fish Oil/Placebo) resting values presented; two-tailed significance

\begin{tabular}{lccc}
\multicolumn{1}{c}{ Variable } & Time & $\begin{array}{c}\text { Time } x \text { Drug } \\
\text { Interaction }\end{array}$ & $\begin{array}{c}\mathbf{N} \text { (fish } \\
\text { oil/placebo) }\end{array}$ \\
\hline SAP $(\mathrm{mmHg})$ & .042 & .585 & $15 / 14$ \\
DAP $(\mathrm{mmHg})$ & .522 & .931 & $15 / 14$ \\
MAP $(\mathrm{mmHg})$ & .221 & .794 & $15 / 14$ \\
HR (beats/min) & .264 & .526 & $15 / 14$ \\
MSNA (bursts/min) & .288 & .381 & $12 / 10$ \\
MSNA (bursts/100hb) & .448 & .388 & $12 / 10$ \\
FBF $(\mathrm{mL} / 100 \mathrm{~mL} / \mathrm{min})$ & .797 & .374 & $14 / 13$ \\
FVR $(\mathrm{mmHg} / \mathrm{mL} / 100 \mathrm{~mL} / \mathrm{min})$ & .207 & .752 & $14 / 13$ \\
FVC $(\mathrm{mL} / 100 \mathrm{~mL} / \mathrm{min} / \mathrm{mmHg})$ & .961 & .545 & $14 / 13$ \\
CBF $(\mathrm{mL} / 100 \mathrm{~mL} / \mathrm{min})$ & .481 & .822 & $14 / 11$ \\
CVR $(\mathrm{mmHg} / \mathrm{mL} / 100 \mathrm{~mL} / \mathrm{min})$ & .923 & .566 & $14 / 11$ \\
CVC $(\mathrm{mL} / 100 \mathrm{~mL} / \mathrm{min} / \mathrm{mmHg})$ & .155 & .686 & $14 / 11$
\end{tabular}


Table D.3. Post-hoc paired t-test of resting SAP $(\mathbf{m m H g})$ prehypertensive group, fish oil.

\begin{tabular}{|c|c|c|c|c|c|c|}
\hline \multirow{2}{*}{ Variable } & \multirow{2}{*}{ Mean } & \multirow{2}{*}{ Std. Dev. } & \multicolumn{2}{|c|}{ 95\% Confidence Interval } & \multirow{2}{*}{ Sig. } & \multirow{2}{*}{$\mathrm{N}$} \\
\hline & & & Lower & Upper & & \\
\hline $\begin{array}{l}\text { Pre Resting vs. } \\
\text { Post Resting }\end{array}$ & -1.76 & 5.42 & -4.76 & 1.24 & .229 & 15 \\
\hline
\end{tabular}

Table D.4. Post-hoc paired t-test of resting SAP $(\mathbf{m m H g})$ prehypertensive group, placebo.

\begin{tabular}{|c|c|c|c|c|c|c|}
\hline \multirow{2}{*}{ Variable } & \multirow{2}{*}{ Mean } & \multirow{2}{*}{ Std. Dev. } & \multicolumn{2}{|c|}{ 95\% Confidence Interval } & \multirow{2}{*}{ Sig. } & \multirow{2}{*}{$\mathrm{N}$} \\
\hline & & & Lower & Upper & & \\
\hline $\begin{array}{l}\text { Pre Resting vs. } \\
\text { Post Resting }\end{array}$ & -2.99 & 5.56 & -6.78 & .795 & .112 & 14 \\
\hline
\end{tabular}

Table D.5. Post-hoc paired t-test of resting FBF ( $\mathrm{mL} / 100 \mathrm{~mL} / \mathrm{min})$ for normotensive group, fish oil.

\begin{tabular}{|c|c|c|c|c|c|c|}
\hline \multirow{2}{*}{ Variable } & \multirow{2}{*}{ Mean } & \multirow{2}{*}{ Std. Dev. } & \multicolumn{2}{|c|}{ 95\% Confidence Interval } & \multirow{2}{*}{ Sig. } & \multirow{2}{*}{$\mathrm{N}$} \\
\hline & & & Lower & Upper & & \\
\hline $\begin{array}{l}\text { Pre Resting vs. } \\
\text { Post Resting }\end{array}$ & .343 & .998 & -.153 & .840 & .082 & 18 \\
\hline
\end{tabular}

Table D.6. Post-hoc paired t-test of resting FBF $(\mathrm{mL} / 100 \mathrm{~mL} / \mathrm{min})$ for normotensive group, placebo.

\begin{tabular}{|c|c|c|c|c|c|c|}
\hline \multirow{2}{*}{ Variable } & \multirow{2}{*}{ Mean } & \multirow{2}{*}{ Std. Dev. } & \multicolumn{2}{|c|}{ 95\% Confidence Interval } & \multirow{2}{*}{ Sig. } & \multirow{2}{*}{$\mathrm{N}$} \\
\hline & & & Lower & Upper & & \\
\hline $\begin{array}{l}\text { Pre Resting vs. } \\
\text { Post Resting }\end{array}$ & .273 & .763 & -.107 & .652 & .074 & 18 \\
\hline
\end{tabular}

Table D.7. Post-hoc paired t-test of resting FVR $(\mathrm{mmHg} / \mathrm{mL} / 100 \mathrm{~mL} / \mathrm{min})$ for normotensive group, fish oil.

\begin{tabular}{|c|c|c|c|c|c|c|}
\hline \multirow{2}{*}{ Variable } & \multirow{2}{*}{ Mean } & \multirow{2}{*}{ Std. Dev. } & \multicolumn{2}{|c|}{$95 \%$ Confidence Interval } & \multirow{2}{*}{ Sig. } & \multirow{2}{*}{$\mathrm{N}$} \\
\hline & & & Lower & Upper & & \\
\hline $\begin{array}{l}\text { Pre Resting vs. } \\
\text { Post Resting }\end{array}$ & -5.25 & -17.68 & -14.04 & 3.54 & .112 & 18 \\
\hline
\end{tabular}


Table D.8. Post-hoc paired t-test of resting FVR $(\mathrm{mmHg} / \mathrm{mL} / 100 \mathrm{~mL} / \mathrm{min})$ for normotensive group, placebo.

\begin{tabular}{|c|c|c|c|c|c|c|}
\hline \multirow{2}{*}{ Variable } & \multirow{2}{*}{ Mean } & \multirow{2}{*}{ Std. Dev. } & \multicolumn{2}{|c|}{$95 \%$ Confidence Interval } & \multirow{2}{*}{ Sig. } & \multirow{2}{*}{$N$} \\
\hline & & & Lower & Upper & & \\
\hline $\begin{array}{l}\text { Pre Resting vs. } \\
\text { Post Resting }\end{array}$ & -7.41 & -13.20 & -13.97 & -.843 & .015 & 18 \\
\hline
\end{tabular}

Table D.9. Post-hoc paired t-test of resting FVC $(\mathrm{mL} / 100 \mathrm{~mL} / \mathrm{min} / \mathrm{mmHg})$ for normotensive group, fish oil.

\begin{tabular}{ccccccc} 
Variable & Mean & Std. Dev. & \multicolumn{2}{c}{$95 \%$ Confidence Interval } & Sig. & N \\
\hline $\begin{array}{c}\text { Pre Resting vs. } \\
\text { Post Resting }\end{array}$ & .005 & .0097 & .0002 & .0098 & .021 & 18
\end{tabular}

Table D.10. Post-hoc paired t-test of resting FVC $(\mathrm{mL} / 100 \mathrm{~mL} / \mathrm{min} / \mathrm{mmHg})$ for normotensive group, placebo.

\begin{tabular}{|c|c|c|c|c|c|c|}
\hline \multirow{2}{*}{ Variable } & \multirow{2}{*}{ Mean } & \multirow{2}{*}{ Std. Dev. } & \multicolumn{2}{|c|}{ 95\% Confidence Interval } & \multirow{2}{*}{ Sig. } & \multirow{2}{*}{$\mathrm{N}$} \\
\hline & & & Lower & Upper & & \\
\hline $\begin{array}{c}\text { Pre Resting vs. } \\
\text { Post Resting }\end{array}$ & .048 & .0100 & -.0002 & .0098 & .029 & 18 \\
\hline
\end{tabular}

Table D.11. Post-hoc paired t-test of resting CVR $(\mathrm{mmHg} / \mathrm{mL} / 100 \mathrm{~mL} / \mathrm{min})$ for normotensive group, fish oil.

\begin{tabular}{ccccccc} 
Variable & Mean & Std. Dev. & \multicolumn{2}{c}{$95 \%$ Confidence Interval } & Sig. & N \\
\hline $\begin{array}{c}\text { Pre Resting vs. } \\
\text { Post Resting }\end{array}$ & -9.12 & -15.67 & -17.47 & -.772 & 16 & .017
\end{tabular}

Table D.12. Post-hoc paired t-test of resting CVR $(\mathrm{mmHg} / \mathrm{mL} / 100 \mathrm{~mL} / \mathrm{min})$ for normotensive group, placebo.

\begin{tabular}{ccccccc} 
Variable & Mean & Std. Dev. & \multicolumn{2}{c}{$95 \%$ Confidence Interval } & Sig. & N \\
\hline $\begin{array}{c}\text { Pre Resting vs. } \\
\text { Post Resting }\end{array}$ & -6.03 & -13.48 & -13.22 & 1.15 & 16 & .047
\end{tabular}


Table D.13. Post-hoc paired t-test of resting CVC $(\mathrm{mL} / 100 \mathrm{~mL} / \mathrm{min} / \mathrm{mmHg})$ for normotensive group, fish oil.

\begin{tabular}{|c|c|c|c|c|c|c|}
\hline \multirow{2}{*}{ Variable } & \multirow{2}{*}{ Mean } & \multirow{2}{*}{ Std. Dev. } & \multicolumn{2}{|c|}{ 95\% Confidence Interval } & \multirow{2}{*}{ Sig. } & \multirow{2}{*}{$\mathrm{N}$} \\
\hline & & & Lower & Upper & & \\
\hline $\begin{array}{l}\text { Pre Resting vs. } \\
\text { Post Resting }\end{array}$ & .0040 & .0088 & -.00073 & .0087 & 16 & .046 \\
\hline
\end{tabular}

Table D.14. Post-hoc paired t-test of resting CVC $(\mathrm{mL} / 100 \mathrm{~mL} / \mathrm{min} / \mathrm{mmHg})$ for normotensive group, placebo.

\begin{tabular}{|c|c|c|c|c|c|c|}
\hline \multirow{2}{*}{ Variable } & \multirow{2}{*}{ Mean } & \multirow{2}{*}{ Std. Dev. } & \multicolumn{2}{|c|}{$95 \%$ Confidence Interval } & \multirow{2}{*}{ Sig. } & \multirow{2}{*}{$\mathrm{N}$} \\
\hline & & & Lower & Upper & & \\
\hline $\begin{array}{l}\text { Pre Resting vs. } \\
\text { Post Resting }\end{array}$ & .0037 & .0074 & -.00023 & .0077 & 16 & .032 \\
\hline
\end{tabular}


Table D.15. Normotensive repeated measures ANOVA of changes in 5 minute average $\triangle$ SAP (mmHg) in Study 2, 2 time (pre/post) vs. 2 condition (base/mental stress) vs. 2 Drug (fish oil/placebo), presented as one-tailed significance.

\begin{tabular}{lc}
\multicolumn{1}{c}{ Interaction } & Base - Mental Stress \\
\hline Time & .383 \\
Time x Drug & .267 \\
Condition & .000 \\
Condition x Drug & .050 \\
Time x Condition & .383 \\
Time x Condition x Drug & .267
\end{tabular}

Table D.16. Prehypertensive repeated measures ANOVA of changes in 5 minute average $\triangle$ SAP (mmHg) in Study 2, 2 time (pre/post) vs. 2 condition (base/mental stress) vs. 2 Drug (fish oil/placebo), presented as one-tailed significance.

\begin{tabular}{lc}
\multicolumn{1}{c}{ Interaction } & Base - Mental Stress \\
\hline Time & .422 \\
Time x Drug & .276 \\
Condition & .000 \\
Condition x Drug & .495 \\
Time x Condition & .422 \\
Time x Condition x Drug & .276
\end{tabular}

Table D.17. Combined-groups repeated measures ANOVA of changes in 5 minute average $\Delta$ SAP (mmHg) in Study 2, 2 time (pre/post) vs. 2 condition (base/mental stress) vs. 2 Drug (fish oil/placebo), presented as one-tailed significance.

\begin{tabular}{lc}
\multicolumn{1}{c}{ Interaction } & Base - Mental Stress \\
\hline Time & .249 \\
Time x Drug & .484 \\
Condition & .347 \\
Condition x Drug & .145 \\
Time x Condition & .249 \\
Time x Condition x Drug & .484
\end{tabular}


Table D.18. Post-hoc paired t-test of condition interaction SAP $(\mathrm{mmHg})$ responses to mental stress for normotensive group, fish oil.

\begin{tabular}{lcccccc}
\multicolumn{1}{c}{ Variable } & Mean & Std. Dev. & \multicolumn{2}{c}{$95 \%$ Confidence Interval } & Sig. & N \\
\hline Pre Base vs. Pre MS & 12.20 & 8.07 & 8.31 & 16.10 & .000 & 19 \\
Post Base vs. Post MS & 12.45 & 7.83 & 8.68 & 16.22 & .000 & 19
\end{tabular}

Table D.19. Post-hoc paired t-test of condition interaction SAP $(\mathrm{mmHg})$ responses to mental stress for normotensive group, placebo.

\begin{tabular}{lcccccc}
\multicolumn{1}{c}{ Variable } & Mean & Std. Dev. & \multicolumn{2}{c}{$95 \%$ Confidence Interval } & Sig. & N \\
& & & Lower & Upper & . & \\
\hline Pre Base vs. Pre MS & 9.32 & 5.00 & 6.91 & 11.72 & .000 & 19 \\
Post Base vs. Post MS & 8.37 & 6.92 & 5.04 & 11.71 & .000 & 19
\end{tabular}

Table D.20. Post-hoc paired t-test of condition interaction SAP $(\mathbf{m m H g})$ responses to mental stress for prehypertensive group, fish oil.

\begin{tabular}{|c|c|c|c|c|c|c|}
\hline \multirow{2}{*}{ Variable } & \multirow{2}{*}{ Mean } & \multirow{2}{*}{ Std. Dev. } & \multicolumn{2}{|c|}{ 95\% Confidence Interval } & \multirow{2}{*}{ Sig. } & \multirow{2}{*}{$\mathrm{N}$} \\
\hline & & & Lower & Upper & & \\
\hline Pre Base vs. Pre MS & 14.57 & 10.10 & 9.00 & 20.16 & .000 & 15 \\
\hline Post Base vs. Post MS & 13.58 & 9.93 & 7.85 & 19.31 & .000 & 14 \\
\hline
\end{tabular}

Table D.21. Post-hoc paired t-test of condition interaction SAP $(\mathrm{mmHg})$ responses to mental stress for prehypertensive group, placebo.

\begin{tabular}{lcccccc}
\multicolumn{1}{c}{ Variable } & Mean & Std. Dev. & \multicolumn{2}{c}{$95 \%$ Confidence Interval } & Sig. & N \\
& & & Lower & Upper & . & \\
\hline Pre Base vs. Pre MS & 13.83 & 9.01 & 8.39 & 19.28 & .000 & 13 \\
Post Base vs. Post MS & 14.42 & 13.7 & 6.14 & 22.70 & .003 & 13
\end{tabular}


Table D.22. Normotensive repeated measures ANOVA of changes in 5 minute average $\triangle$ DAP (mmHg) in Study 2, 2 time (pre/post) vs. 2 condition (base/mental stress) vs. 2 Drug (fish oil/placebo), presented as one-tailed significance.

\begin{tabular}{lc}
\multicolumn{1}{c}{ Interaction } & Base - Mental Stress \\
\hline Time & .254 \\
Time x Drug & .101 \\
Condition & .000 \\
Condition x Drug & .019 \\
Time x Condition & .254 \\
Time x Condition x Drug & .101
\end{tabular}

Table D.23. Prehypertensive repeated measures ANOVA of changes in 5 minute average $\triangle D A P$ (mmHg) in Study 2, 2 time (pre/post) vs. 2 condition (base/mental stress) vs. 2 Drug (fish oil/placebo), presented as one-tailed significance.

\begin{tabular}{lc}
\multicolumn{1}{c}{ Interaction } & Base - Mental Stress \\
\hline Time & .189 \\
Time x Drug & .491 \\
Condition & .000 \\
Condition x Drug & .428 \\
Time x Condition & .189 \\
Time x Condition x Drug & .491
\end{tabular}

Table D.24. Combined-groups repeated measures ANOVA of changes in 5 minute average $\triangle \mathrm{DAP}(\mathrm{mmHg})$ in Study 2, 2 time (pre/post) vs. 2 condition (base/mental stress) vs. 2 Drug (fish oil/placebo), presented as one-tailed significance.

\begin{tabular}{lc}
\multicolumn{1}{c}{ Interaction } & Base - Mental Stress \\
\hline Time & .262 \\
Time x Drug & .173 \\
Condition & .215 \\
Condition x Drug & .175 \\
Time x Condition & .262 \\
Time x Condition x Drug & .173
\end{tabular}


Table D.25. Post-hoc paired t-test of condition interaction DAP $(\mathbf{m m H g})$ responses to mental stress for normotensive group, fish oil.

\begin{tabular}{|c|c|c|c|c|c|c|}
\hline \multirow{2}{*}{ Variable } & \multirow{2}{*}{ Mean } & \multirow{2}{*}{ Std. Dev. } & \multicolumn{2}{|c|}{ 95\% Confidence Interval } & \multirow{2}{*}{ Sig. } & \multirow{2}{*}{$\mathrm{N}$} \\
\hline & & & Lower & Upper & & \\
\hline Pre Base vs. Pre MS & 9.66 & 3.75 & 7.86 & 11.47 & .000 & 19 \\
\hline Post Base vs. Post MS & 10.70 & 3.27 & 9.12 & 12.28 & .000 & 19 \\
\hline
\end{tabular}

Table D.26. Post-hoc paired t-test of condition interaction DAP $(\mathbf{m m H g})$ responses to mental stress for normotensive group, placebo.

\begin{tabular}{lcccccc}
\multicolumn{1}{c}{ Variable } & Mean & Std. Dev. & \multicolumn{2}{c}{$95 \%$ Confidence Interval } & Sig. & N \\
& & & Lower & Upper & Sig & \\
\hline Pre Base vs. Pre MS & 8.15 & 3.37 & 6.53 & 9.78 & .000 & 19 \\
Post Base vs. Post MS & 7.82 & 3.73 & 6.03 & 9.62 & .000 & 19
\end{tabular}

Table D.27. Post-hoc paired t-test of condition interaction DAP $(\mathbf{m m H g})$ responses to mental stress for prehypertensive group, fish oil.

\begin{tabular}{lcccccc}
\multicolumn{1}{c}{ Variable } & Mean & Std. Dev. & \multicolumn{2}{c}{$95 \%$ Confidence Interval } & Sig. & N \\
\hline Pre Base vs. Pre MS & 10.49 & 5.50 & 7.32 & 13.67 & .000 & 15 \\
Post Base vs. Post MS & 9.89 & 4.89 & 7.07 & 12.71 & .000 & 14
\end{tabular}

Table D.28. Post-hoc paired t-test of condition interaction DAP $(\mathbf{m m H g})$ responses to mental stress for prehypertensive group, placebo.

\begin{tabular}{lcccccc}
\multicolumn{1}{c}{ Variable } & Mean & Std. Dev. & \multicolumn{2}{c}{$95 \%$ Confidence Interval } & Sig. & N \\
& & & Lower & Upper & . & \\
\hline Pre Base vs. Pre MS & 10.87 & 4.96 & 7.87 & 13.86 & .000 & 13 \\
Post Base vs. Post MS & 10.20 & 5.96 & 6.59 & 13.80 & .000 & 13
\end{tabular}


Table D.29. Normotensive repeated measures ANOVA of changes in 5 minute average $\triangle M A P$ (mmHg) in Study 2, 2 time (pre/post) vs. 2 condition (base/mental stress) vs. 2 Drug (fish oil/placebo), presented as one-tailed significance.

\begin{tabular}{lc}
\multicolumn{1}{c}{ Interaction } & Base - Mental Stress \\
\hline Time & .483 \\
Time x Drug & .150 \\
Condition & .000 \\
Condition x Drug & .026 \\
Time x Condition & .483 \\
Time x Condition x Drug & .150
\end{tabular}

Table D.30. Prehypertensive repeated measures ANOVA of changes in 5 minute average $\triangle M A P$ (mmHg) in Study 2, 2 time (pre/post) vs. 2 condition (base/mental stress) vs. 2 Drug (fish oil/placebo), presented as one-tailed significance.

\begin{tabular}{lc}
\multicolumn{1}{c}{ Interaction } & Base - Mental Stress \\
\hline Time & .079 \\
Time x Drug & .194 \\
Condition & .000 \\
Condition x Drug & .382 \\
Time x Condition & .079 \\
Time x Condition x Drug & .194
\end{tabular}

Table D.31. Combined-groups repeated measures ANOVA of changes in 5 minute average $\triangle$ MAP (mmHg) in Study 2, 2 time (pre/post) vs. 2 condition (base/mental stress) vs. 2 Drug (fish oil/placebo), presented as one-tailed significance.

\begin{tabular}{lc}
\multicolumn{1}{c}{ Interaction } & Base - Mental Stress \\
\hline Time & .204 \\
Time x Drug & .410 \\
Condition & .397 \\
Condition x Drug & .064 \\
Time x Condition & .204 \\
Time x Condition x Drug & .410
\end{tabular}


Table D.32. Post-hoc paired t-test of condition interaction MAP $(\mathbf{m m H g})$ responses to mental stress for normotensive group, fish oil.

\begin{tabular}{lcccccc}
\multicolumn{1}{c}{ Variable } & Mean & Std. Dev. & $\begin{array}{c}95 \% \text { Confidence Interval } \\
\text { Lower }\end{array}$ & Upper & Sig. & N \\
\hline Pre Base vs. Pre MS & 12.03 & 5.42 & 9.42 & 14.64 & .000 & 19 \\
Post Base vs. Post MS & 12.82 & 4.90 & 10.46 & 15.18 & .000 & 19
\end{tabular}

Table D.33. Post-hoc paired t-test of condition interaction MAP $(\mathbf{m m H g})$ responses to mental stress for normotensive group, placebo.

\begin{tabular}{lcccccc}
\multicolumn{1}{c}{ Variable } & Mean & Std. Dev. & \multicolumn{2}{c}{$95 \%$ Confidence Interval } & Sig. & N \\
& & & Lower & Upper & S & \\
\hline Pre Base vs. Pre MS & 9.99 & 4.26 & 7.94 & 12.04 & .000 & 19 \\
Post Base vs. Post MS & 9.26 & 4.78 & 6.95 & 11.57 & .000 & 19
\end{tabular}

Table D.34. Post-hoc paired t-test of condition interaction MAP $(\mathbf{m m H g})$ responses to mental stress for prehypertensive group, fish oil.

\begin{tabular}{lcccccc}
\multicolumn{1}{c}{ Variable } & Mean & Std. Dev. & \multicolumn{2}{c}{$95 \%$ Confidence Interval } & Sig. & N \\
& & & Lower & Upper & Sig. & \\
\hline Pre Base vs. Pre MS & 14.67 & 7.17 & 10.72 & 18.67 & .000 & 15 \\
Post Base vs. Post MS & 13.09 & 6.25 & 9.48 & 16.71 & .000 & 14
\end{tabular}

Table D.35. Post-hoc paired t-test of condition interaction MAP $(\mathbf{m m H g})$ responses to mental stress for prehypertensive group, placebo.

\begin{tabular}{lcccccc}
\multicolumn{1}{c}{ Variable } & Mean & Std. Dev. & \multicolumn{2}{c}{$95 \%$ Confidence Interval } & Sig. & N \\
& & & Lower & Upper & . & \\
\hline Pre Base vs. Pre MS & 13.52 & 5.70 & 10.08 & 16.97 & .000 & 13 \\
Post Base vs. Post MS & 13.04 & 8.19 & 8.09 & 17.99 & .000 & 13
\end{tabular}


Table D.36. Normotensive repeated measures ANOVA of changes in 5 minute average $\Delta \mathbf{H R}$ (beats/min) in Study 2, 2 time (pre/post) vs. 2 condition (base/mental stress) vs. 2 Drug (fish oil/placebo), presented as one-tailed significance.

\begin{tabular}{lc}
\multicolumn{1}{c}{ Interaction } & Base - Mental Stress \\
\hline Time & .056 \\
Time x Drug & .007 \\
Condition & .000 \\
Condition x Drug & .001 \\
Time x Condition & .056 \\
Time x Condition x Drug & .007
\end{tabular}

Table D.37. Prehypertensive repeated measures ANOVA of changes in 5 minute average $\Delta H R$ (beats/min) in Study 2, 2 time (pre/post) vs. 2 condition (base/mental stress) vs. 2 Drug (fish oil/placebo), presented as one-tailed significance.

\begin{tabular}{lc}
\multicolumn{1}{c}{ Interaction } & Base - Mental Stress \\
\hline Time & .093 \\
Time x Drug & .189 \\
Condition & .000 \\
Condition x Drug & .299 \\
Time x Condition & .093 \\
Time x Condition x Drug & .189
\end{tabular}

Table D.38. Combined-groups repeated measures ANOVA of changes in 5 minute average $\Delta H R$ (beats/min) in Study 2, 2 time (pre/post) vs. 2 condition (base/mental stress) vs. 2 Drug (fish oil/placebo), presented as one-tailed significance.

\begin{tabular}{lc}
\multicolumn{1}{c}{ Interaction } & Base - Mental Stress \\
\hline Time & .351 \\
Time x Drug & .009 \\
Condition & .026 \\
Condition x Drug & .019 \\
Time x Condition & .351 \\
Time x Condition x Drug & .009
\end{tabular}


Table D.39. Post-hoc paired t-test of condition interaction $\quad$ HR (beats $/ \mathrm{min}$ ) responses to mental stress for normotensive group, fish oil.

\begin{tabular}{lcccccc}
\multicolumn{1}{c}{ Variable } & Mean & Std. Dev. & \multicolumn{2}{c}{$95 \%$ Confidence Interval } & Sig. & N \\
\hline Pre Base vs. Pre MS & 24.72 & 10.61 & 19.61 & 29.83 & .000 & 19 \\
Post Base vs. Post MS & 21.61 & 11.27 & 16.17 & 27.04 & .000 & 19 \\
Pre MS vs. Post MS & -3.11 & 5.91 & -5.96 & -.263 & .034 & 19
\end{tabular}

Table D.40. Post-hoc paired t-test of condition interaction $\quad$ HR (beats $/ \mathrm{min}$ ) responses to mental stress for normotensive group, placebo.

\begin{tabular}{lcccccc}
\multicolumn{1}{c}{ Variable } & Mean & Std. Dev. & \multicolumn{2}{c}{$95 \%$ Confidence Interval } & Sig. & N \\
\hline Pre Base vs. Pre MS & 12.86 & 5.81 & 10.06 & 15.67 & .000 & 19 \\
Post Base vs. Post MS & 13.55 & 7.21 & 10.07 & 17.02 & .000 & 19 \\
Pre MS vs. Post MS & .682 & 2.72 & -.630 & 1.99 & .289 & 19
\end{tabular}

Table D.41. Post-hoc paired t-test of condition interaction HR (beats/min) responses to mental stress for prehypertensive group, fish oil.

\begin{tabular}{lcccccc}
\multicolumn{1}{c}{ Variable } & Mean & Std. Dev. & \multicolumn{2}{c}{$95 \%$ Confidence Interval } & Sig. & N \\
& & & Lower & Upper & & \\
\hline Pre Base vs. Pre MS & 17.52 & 7.26 & 13.50 & 21.54 & .000 & 15 \\
Post Base vs. Post MS & 15.57 & 6.74 & 11.68 & 19.46 & .000 & 14
\end{tabular}

Table D.42. Post-hoc paired t-test of condition interaction HR (beats/min) responses to mental stress for prehypertensive group, placebo.

\begin{tabular}{|c|c|c|c|c|c|c|}
\hline \multirow{2}{*}{ Variable } & \multirow{2}{*}{ Mean } & \multirow{2}{*}{ Std. Dev. } & \multicolumn{2}{|c|}{ 95\% Confidence Interval } & \multirow{2}{*}{ Sig. } & \multirow{2}{*}{$\mathrm{N}$} \\
\hline & & & Lower & Upper & & \\
\hline Pre Base vs & 19.32 & 12.62 & 12.03 & 26.61 & .000 & 13 \\
\hline Post Base vs. Post MS & 18.47 & 11.38 & 11.60 & 25.35 & .000 & 13 \\
\hline
\end{tabular}

Table D.43. Post-hoc paired t-test of condition interaction $\quad$ HR (beats $/ \mathrm{min}$ ) responses to mental stress for combined-group, fish oil.

\begin{tabular}{lcccccc}
\multicolumn{1}{c}{ Variable } & Mean & Std. Dev. & \multicolumn{2}{c}{$95 \%$ Confidence Interval } & Sig. & N \\
\hline Pre Base vs. Pre MS & 21.54 & 9.84 & 18.11 & 24.98 & .000 & 34 \\
Post Base vs. Post MS & 19.05 & 9.95 & 15.52 & 22.58 & .000 & 33 \\
Pre MS vs. Post MS & -2.80 & 5.18 & -4.63 & -.959 & .002 & 33
\end{tabular}


Table D.44. Post-hoc paired t-test of condition interaction $\quad$ HR (beats $/ \mathrm{min}$ ) responses to mental stress for combined-group, placebo.

\begin{tabular}{lcccccc}
\multicolumn{1}{c}{ Variable } & Mean & Std. Dev. & \multicolumn{2}{c}{$95 \%$ Confidence Interval } & Sig. & N \\
\hline Pre Base vs. Pre MS & 15.60 & 9.71 & 12.16 & 19.05 & .000 & 33 \\
Post Base vs. Post MS & 15.55 & 9.29 & 12.20 & 18.90 & .000 & 32 \\
Pre MS vs. Post MS & .207 & 4.60 & -1.45 & 1.87 & .400 & 32
\end{tabular}

Table D.45. Combined-groups repeated measures ANOVA of changes in 5 minute average $\triangle$ MSNA (bursts/min) in Study 2, 2 time (pre/post) vs. 2 condition (base/mental stress) vs. 2 Drug (fish oil/placebo), presented as one-tailed significance.

\begin{tabular}{lc}
\multicolumn{1}{c}{ Interaction } & Base - Mental Stress \\
\hline Time & .326 \\
Time x Drug & .398 \\
Condition & .010 \\
Condition x Drug & .449 \\
Time x Condition & .326 \\
Time x Condition x Drug & .398
\end{tabular}

Table D.46. Post-hoc paired t-test of condition interaction $\triangle$ MSNA (bursts/min) responses to mental stress for combined-group, fish oil.

\begin{tabular}{lcccccc}
\multicolumn{1}{c}{ Variable } & Mean & Std. Dev. & \multicolumn{2}{c}{$95 \%$ Confidence Interval } & Sig. & N \\
& & & Lower & Upper & & \\
\hline Pre Base vs. Pre MS & 5.78 & 6.13 & 1.88 & 9.68 & .004 & 12 \\
Post Base vs. Post MS & 4.82 & 4.52 & 1.95 & 7.69 & .002 & 12
\end{tabular}

Table D.47. Post-hoc paired t-test of condition interaction $\triangle$ MSNA (bursts/min) responses to mental stress for combined-group, placebo.

\begin{tabular}{lcccccc}
\multicolumn{1}{c}{ Variable } & Mean & Std. Dev. & \multicolumn{2}{c}{$95 \%$ Confidence Interval } & Sig. & N \\
& & & Lower & Upper & Sig. & \\
\hline Pre Base vs. Pre MS & 6.00 & 4.86 & 3.50 & 8.50 & .000 & 17 \\
Post Base vs. Post MS & 5.89 & 5.00 & 3.32 & 8.46 & .000 & 17
\end{tabular}


Table D.48. Combined-groups repeated measures ANOVA of changes in 5 minute average $\triangle$ MSNA (bursts/100hb) in Study 2, 2 time (pre/post) vs. 2 condition (base/mental stress) vs. 2 Drug (fish oil/placebo), presented as one-tailed significance.

\begin{tabular}{lc}
\multicolumn{1}{c}{ Interaction } & Base - Mental Stress \\
\hline Time & .398 \\
Time x Drug & .364 \\
Condition & .024 \\
Condition x Drug & .457 \\
Time x Condition & .398 \\
Time x Condition x Drug & .364
\end{tabular}

Table D.49. Post-hoc paired t-test of condition interaction $\Delta$ MSNA (bursts/100hb) responses to mental stress for combined-group, fish oil.

\begin{tabular}{|c|c|c|c|c|c|c|}
\hline \multirow{2}{*}{ Variable } & \multirow{2}{*}{ Mean } & \multirow{2}{*}{ Std. Dev. } & \multicolumn{2}{|c|}{$95 \%$ Confidence Interval } & \multirow{2}{*}{ Sig. } & \multirow{2}{*}{$\mathrm{N}$} \\
\hline & & & Lower & Upper & & \\
\hline Pre Base vs. Pre MS & 4.19 & 9.59 & -1.91 & 10.28 & .080 & 12 \\
\hline Post Base vs. Post MS & 3.13 & 6.94 & -1.29 & 7.54 & .079 & 12 \\
\hline
\end{tabular}

Table D.50. Post-hoc paired t-test of condition interaction $\Delta$ MSNA (bursts/100hb) responses to mental stress for combined-group, placebo.

\begin{tabular}{|c|c|c|c|c|c|c|}
\hline \multirow{2}{*}{ Variable } & \multirow{2}{*}{ Mean } & \multirow{2}{*}{ Std. Dev. } & \multicolumn{2}{|c|}{$95 \%$ Confidence Interval } & \multirow{2}{*}{ Sig. } & \multirow{2}{*}{$\mathrm{N}$} \\
\hline & & & Lower & Upper & & \\
\hline Pre Base vs. Pre MS & 3.99 & 7.05 & .368 & 7.62 & .016 & 17 \\
\hline Post Base vs. Post MS & 3.98 & 6.03 & .883 & 7.08 & .008 & 17 \\
\hline
\end{tabular}

Table D.51. Combined-groups repeated measures ANOVA of changes in 5 minute average $\Delta$ Total MSNA (arbitrary units) in Study 2, 2 time (pre/post) vs. 2 condition (base/mental stress) vs. 2 Drug (fish oil/placebo), presented as one-tailed significance.

\begin{tabular}{lc}
\multicolumn{1}{c}{ Interaction } & Base - Mental Stress \\
\hline Time & .102 \\
Time x Drug & .041 \\
Condition & .113 \\
Condition x Drug & .459 \\
Time x Condition & .102 \\
Time x Condition x Drug & .041
\end{tabular}


Table D.52. Post-hoc paired t-test of condition interaction $\Delta$ Total MSNA (arbitrary units) responses to mental stress for combined-group, fish oil.

\begin{tabular}{lcccccc}
\multicolumn{1}{c}{ Variable } & Mean & Std. Dev. & \multicolumn{2}{c}{$95 \%$ Confidence Interval } & Sig. & N \\
\hline Pre Base vs. Pre MS & 9627.9 & 7219.5 & 4078.5 & 15177.3 & .002 & 9 \\
Post Base vs. Post MS & 3830.2 & 3087.8 & 1456.7 & 6203.6 & .003 & 9 \\
Pre MS vs. Post MS & -5797.7 & 6576.1 & -10852.6 & -742.9 & .015 & 9
\end{tabular}

Table D.53. Post-hoc paired t-test of condition interaction $\Delta$ MSNA (bursts/100hb) responses to mental stress for combined-group, placebo.

\begin{tabular}{|c|c|c|c|c|c|c|}
\hline \multirow{2}{*}{ Variable } & \multirow{2}{*}{ Mean } & \multirow{2}{*}{ Std. Dev. } & \multicolumn{2}{|c|}{ 95\% Confidence Interval } & \multirow{2}{*}{ Sig. } & \multirow{2}{*}{$\mathrm{N}$} \\
\hline & & & Lower & Upper & & \\
\hline Pre Base vs. Pre MS & 6860.4 & 5474.9 & 3699.3 & 10021.6 & .000 & 14 \\
\hline Post Base vs. Post MS & 6302.3 & 6589.6 & 1890.5 & 10714.2 & .004 & 14 \\
\hline Pre MS vs. Post MS & -558.10 & 6589.6 & -4362.8 & 3246.6 & .378 & 14 \\
\hline
\end{tabular}


Table D.54. Normotensive repeated measures ANOVA of changes in 5 minute average $\triangle F B F$ (\%) in Study 2, 2 time (pre/post) vs. 2 condition (base/mental stress) vs. 2 Drug (fish oil/placebo), presented as one-tailed significance.

\begin{tabular}{lc}
\multicolumn{1}{c}{ Interaction } & Base - Mental Stress \\
\hline Time & .337 \\
Time x Drug & .497 \\
Condition & .000 \\
Condition x Drug & .003 \\
Time x Condition & .337 \\
Time x Condition x Drug & .497
\end{tabular}

Table D.55. Prehypertensive repeated measures ANOVA of changes in 5 minute average $\Delta F B F$ (\%) in Study 2, 2 time (pre/post) vs. 2 condition (base/mental stress) vs. 2 Drug (fish oil/placebo), presented as one-tailed significance.

\begin{tabular}{lc}
\multicolumn{1}{c}{ Interaction } & Base - Mental Stress \\
\hline Time & .043 \\
Time x Drug & .491 \\
Condition & .000 \\
Condition x Drug & .396 \\
Time x Condition & .043 \\
Time x Condition x Drug & .491
\end{tabular}

Table D.56. Combined-groups repeated measures ANOVA of changes in 5 minute average $\triangle$ FBF (\%) in Study 2, 2 time (pre/post) vs. 2 condition (base/mental stress) vs. 2 Drug (fish oil/placebo), presented as one-tailed significance.

\begin{tabular}{lc}
\multicolumn{1}{c}{ Interaction } & Base - Mental Stress \\
\hline Time & .073 \\
Time x Drug & .478 \\
Condition & .000 \\
Condition x Drug & .011 \\
Time x Condition & .073 \\
Time x Condition x Drug & .478
\end{tabular}


Table D.57. Post-hoc paired t-test of condition interaction FBF (\%) responses to mental stress for normotensive group, fish oil.

\begin{tabular}{lcccccc}
\multicolumn{1}{c}{ Variable } & Mean & Std. Dev. & \multicolumn{2}{c}{$95 \%$ Confidence Interval } & Sig. & N \\
& & & Lower & Upper & Spe & \\
\hline Pre Base vs. Pre MS & 109.1 & 62.65 & 75.67 & 142.44 & .000 & 16 \\
Post Base vs. Post MS & 104.1 & 72.23 & 65.66 & 142.63 & .000 & 16
\end{tabular}

Table D.58. Post-hoc paired t-test of condition interaction FBF (\%) responses to mental stress for normotensive group, placebo.

\begin{tabular}{lcccccc}
\multicolumn{1}{c}{ Variable } & Mean & Std. Dev. & \multicolumn{2}{c}{$95 \%$ Confidence Interval } & Sig. & N \\
& & & Lower & Upper & . & \\
\hline Pre Base vs. Pre MS & 58.86 & 45.64 & 34.54 & 83.18 & .000 & 16 \\
Post Base vs. Post MS & 54.11 & 40.25 & 32.67 & 75.56 & .000 & 16
\end{tabular}

Table D.59. Post-hoc paired t-test of condition interaction FBF (\%) responses to mental stress for prehypertensive group, fish oil.

\begin{tabular}{|c|c|c|c|c|c|c|}
\hline \multirow{2}{*}{ Variable } & \multirow{2}{*}{ Mean } & \multirow{2}{*}{ Std. Dev. } & \multicolumn{2}{|c|}{ 95\% Confidence Interval } & \multirow{2}{*}{ Sig. } & \multirow{2}{*}{$\mathrm{N}$} \\
\hline & & & Lower & Upper & & \\
\hline Pre Base vs. Pre MS & 71.76 & 51.86 & 40.43 & 103.1 & .000 & 13 \\
\hline Post Base vs. Post MS & 50.36 & 45.11 & 23.10 & 77.62 & .002 & 13 \\
\hline
\end{tabular}

Table D.60. Post-hoc paired t-test of condition interaction FBF (\%) responses to mental stress for prehypertensive group, placebo.

\begin{tabular}{lcccccc}
\multicolumn{1}{c}{ Variable } & Mean & Std. Dev. & \multicolumn{2}{c}{$95 \%$ Confidence Interval } & Sig. & N \\
& & & Lower & Upper & . & \\
\hline Pre Base vs. Pre MS & 67.56 & 52.38 & 30.08 & 105.03 & .003 & 10 \\
Post Base vs. Post MS & 45.57 & 46.34 & 12.43 & 78.72 & .013 & 10
\end{tabular}


Table D.61. Normotensive repeated measures ANOVA of changes in 5 minute average $\mathbf{A F V R}$ (\%) in Study 2, 2 time (pre/post) vs. 2 condition (base/mental stress) vs. 2 Drug (fish oil/placebo), presented as one-tailed significance.

\begin{tabular}{lc}
\multicolumn{1}{c}{ Interaction } & Base - Mental Stress \\
\hline Time & .336 \\
Time x Drug & .497 \\
Condition & .000 \\
Condition x Drug & .003 \\
Time x Condition & .336 \\
Time x Condition x Drug & .497
\end{tabular}

Table D.62. Prehypertensive repeated measures ANOVA of changes in 5 minute average $\Delta F V R$ (\%) in Study 2, 2 time (pre/post) vs. 2 condition (base/mental stress) vs. 2 Drug (fish oil/placebo), presented as one-tailed significance.

\begin{tabular}{lc}
\multicolumn{1}{c}{ Interaction } & Base - Mental Stress \\
\hline Time & .081 \\
Time x Drug & .349 \\
Condition & .000 \\
Condition x Drug & .351 \\
Time x Condition & .081 \\
Time x Condition x Drug & .349
\end{tabular}

Table D.63. Combined-groups repeated measures ANOVA of changes in 5 minute average $\Delta$ FVR (\%) in Study 2, 2 time (pre/post) vs. 2 condition (base/mental stress) vs. 2 Drug (fish oil/placebo), presented as one-tailed significance.

\begin{tabular}{lc}
\multicolumn{1}{c}{ Interaction } & Base - Mental Stress \\
\hline Time & .024 \\
Time x Drug & .366 \\
Condition & .000 \\
Condition x Drug & .127 \\
Time x Condition & .024 \\
Time x Condition x Drug & .366
\end{tabular}


Table D.64. Post-hoc paired t-test of condition interaction FVR (\%) responses to mental stress for normotensive group, fish oil.

\begin{tabular}{lcccccc}
\multicolumn{1}{c}{ Variable } & Mean & Std. Dev. & \multicolumn{2}{c}{$95 \%$ Confidence Interval } & Sig. & N \\
\hline Pre Base vs. Pre MS & -37.88 & 20.19 & -48.64 & -27.12 & .000 & 16 \\
Post Base vs. Post MS & -35.63 & 19.71 & -46.13 & -25.13 & .000 & 16
\end{tabular}

Table D.65. Post-hoc paired t-test of condition interaction FVR (\%) responses to mental stress for normotensive group, placebo.

\begin{tabular}{lcccccc}
\multicolumn{1}{c}{ Variable } & Mean & Std. Dev. & \multicolumn{2}{c}{$95 \%$ Confidence Interval } & Sig. & N \\
& & & Lower & Upper & & \\
\hline Pre Base vs. Pre MS & -25.64 & 18.35 & -35.42 & 15.86 & .000 & 16 \\
Post Base vs. Post MS & -20.08 & 19.38 & -30.41 & -9.75 & .000 & 16
\end{tabular}

Table D.66. Post-hoc paired t-test of condition interaction FVR (\%) responses to mental stress for prehypertensive group, fish oil.

\begin{tabular}{|c|c|c|c|c|c|c|}
\hline \multirow{2}{*}{ Variable } & \multirow{2}{*}{ Mean } & \multirow{2}{*}{ Std. Dev. } & \multicolumn{2}{|c|}{ 95\% Confidence Interval } & \multirow{2}{*}{ Sig. } & \multirow{2}{*}{$\mathrm{N}$} \\
\hline & & & Lower & Upper & & \\
\hline Pre Base vs. Pre MS & -18.07 & 24.97 & -33.94 & -2.21 & .029 & 13 \\
\hline Post Base vs. Post MS & -12.63 & 28.24 & -30.57 & 5.32 & .150 & 13 \\
\hline
\end{tabular}

Table D.67. Post-hoc paired t-test of condition interaction FVR (\%) responses to mental stress for prehypertensive group, placebo.

\begin{tabular}{lcccccc}
\multicolumn{1}{c}{ Variable } & Mean & Std. Dev. & \multicolumn{2}{c}{$95 \%$ Confidence Interval } & Sig. & N \\
& & & Lower & Upper & . & \\
\hline Pre Base vs. Pre MS & -23.95 & 21.74 & -39.14 & -8.04 & .007 & 10 \\
Post Base vs. Post MS & -14.07 & 19.67 & -28.14 & .006 & .050 & 10
\end{tabular}


Table D.68. Normotensive repeated measures ANOVA of changes in 5 minute average $\Delta$ FVC (\%) in Study 2, 2 time (pre/post) vs. 2 condition (base/mental stress) vs. 2 Drug (fish oil/placebo), presented as one-tailed significance.

\begin{tabular}{lc}
\multicolumn{1}{c}{ Interaction } & Base - Mental Stress \\
\hline Time & .146 \\
Time x Drug & .326 \\
Condition & .000 \\
Condition x Drug & .012 \\
Time x Condition & .146 \\
Time x Condition x Drug & .326
\end{tabular}

Table D.69. Prehypertensive repeated measures ANOVA of changes in 5 minute average $\Delta F V C$ (\%) in Study 2, 2 time (pre/post) vs. 2 condition (base/mental stress) vs. 2 Drug (fish oil/placebo), presented as one-tailed significance.

\begin{tabular}{lc}
\multicolumn{1}{c}{ Interaction } & Base - Mental Stress \\
\hline Time & .245 \\
Time x Drug & .413 \\
Condition & .000 \\
Condition x Drug & .006 \\
Time x Condition & .244 \\
Time x Condition x Drug & .413
\end{tabular}

Table D.70. Combined-groups repeated measures ANOVA of changes in 5 minute average $\Delta$ FVC (\%) in Study 2, 2 time (pre/post) vs. 2 condition (base/mental stress) vs. 2 Drug (fish oil/placebo), presented as one-tailed significance.

\begin{tabular}{lc}
\multicolumn{1}{c}{ Interaction } & Base - Mental Stress \\
\hline Time & .066 \\
Time x Drug & .407 \\
Condition & .000 \\
Condition x Drug & .025 \\
Time x Condition & .066 \\
Time x Condition x Drug & .407
\end{tabular}


Table D.71. Post-hoc paired t-test of condition interaction FVC (\%) responses to mental stress for normotensive group, fish oil.

\begin{tabular}{lcccccc}
\multicolumn{1}{c}{ Variable } & Mean & Std. Dev. & 95\% Confidence Interval & Sig. & N \\
& & & Lower & Upper & Spe & \\
\hline Pre Base vs. Pre MS & 86.02 & 55.06 & 56.68 & 115.37 & .000 & 16 \\
Post Base vs. Post MS & 78.42 & 64.12 & 44.25 & 112.59 & .000 & 16
\end{tabular}

Table D.72. Post-hoc paired t-test of condition interaction FVC (\%) responses to mental stress for normotensive group, placebo.

\begin{tabular}{lcccccc}
\multicolumn{1}{c}{ Variable } & Mean & Std. Dev. & \multicolumn{2}{c}{$95 \%$ Confidence Interval } & Sig. & N \\
& & & Lower & Upper & Sp. & \\
\hline Pre Base vs. Pre MS & 45.13 & 39.66 & 24.00 & 66.26 & .000 & 16 \\
Post Base vs. Post MS & 39.29 & 36.54 & 19.81 & 58.76 & .001 & 16
\end{tabular}

Table D.73. Post-hoc paired t-test of condition interaction FVC (\%) responses to mental stress for prehypertensive group, fish oil.

\begin{tabular}{lcccccc}
\multicolumn{1}{c}{ Variable } & Mean & Std. Dev. & \multicolumn{2}{c}{$95 \%$ Confidence Interval } & Sig. & N \\
& & & Lower & Upper & . & \\
\hline Pre Base vs. Pre MS & 48.94 & 45.30 & 21.57 & 76.31 & .002 & 13 \\
Post Base vs. Post MS & 30.75 & 38.94 & 7.22 & 54.29 & .015 & 13
\end{tabular}

Table D.74. Post-hoc paired t-test of condition interaction FVC (\%) responses to mental stress for prehypertensive group, placebo.

\begin{tabular}{lcccccc}
\multicolumn{1}{c}{ Variable } & Mean & Std. Dev. & \multicolumn{2}{c}{$95 \%$ Confidence Interval } & Sig. & N \\
& & & Lower & Upper & . & \\
\hline Pre Base vs. Pre MS & 45.38 & 44.09 & 13.84 & 76.92 & .010 & 10 \\
Post Base vs. Post MS & 31.21 & 43.49 & .102 & 62.32 & .049 & 10
\end{tabular}


Table D.75. Normotensive repeated measures ANOVA of changes in 5 minute average $\Delta$ CBF (\%) in Study 2, 2 time (pre/post) vs. 2 condition (base/mental stress) vs. 2 Drug (fish oil/placebo), presented as one-tailed significance.

\begin{tabular}{lc}
\multicolumn{1}{c}{ Interaction } & Base - Mental Stress \\
\hline Time & .007 \\
Time x Drug & .025 \\
Condition & .000 \\
Condition x Drug & .080 \\
Time x Condition & .007 \\
Time x Condition x Drug & .025
\end{tabular}

Table D.76. Prehypertensive repeated measures ANOVA of changes in 5 minute average $\Delta \mathbf{C B F}$ (\%) in Study 2, 2 time (pre/post) vs. 2 condition (base/mental stress) vs. 2 Drug (fish oil/placebo), presented as one-tailed significance.

\begin{tabular}{lc}
\multicolumn{1}{c}{ Interaction } & Base - Mental Stress \\
\hline Time & .145 \\
Time x Drug & .166 \\
Condition & .000 \\
Condition x Drug & .448 \\
Time x Condition & .145 \\
Time x Condition x Drug & .332
\end{tabular}

Table D.77. Combined-groups repeated measures ANOVA of changes in 5 minute average $\Delta$ CBF (\%) in Study 2, 2 time (pre/post) vs. 2 condition (base/mental stress) vs. 2 Drug (fish oil/placebo), presented as one-tailed significance.

\begin{tabular}{lc}
\multicolumn{1}{c}{ Interaction } & Base - Mental Stress \\
\hline Time & .005 \\
Time x Drug & .018 \\
Condition & .000 \\
Condition x Drug & .110 \\
Time x Condition & .005 \\
Time x Condition x Drug & .018
\end{tabular}


Table D.78. Post-hoc paired t-test of condition interaction CBF (\%) responses to mental stress for normotensive group, fish oil.

\begin{tabular}{lcccccc}
\multicolumn{1}{c}{ Variable } & Mean & Std. Dev. & \multicolumn{2}{c}{$95 \%$ Confidence Interval } & Sig. & N \\
\hline Pre Base vs. Pre MS & 52.99 & 45.06 & 28.03 & 77.94 & .000 & 15 \\
Post Base vs. Post MS & 32.87 & 31.65 & 15.34 & 50.39 & .001 & 15 \\
Pre MS vs. Post MS & -20.12 & 26.57 & -34.83 & -5.41 & .005 & 15
\end{tabular}

Table D.79. Post-hoc paired t-test of condition interaction CBF (\%) responses to mental stress for normotensive group, placebo.

\begin{tabular}{|c|c|c|c|c|c|c|}
\hline \multirow{2}{*}{ Variable } & \multirow{2}{*}{ Mean } & \multirow{2}{*}{ Std. Dev. } & \multicolumn{2}{|c|}{$95 \%$ Confidence Interval } & \multirow{2}{*}{ Sig. } & \multirow{2}{*}{$\mathrm{N}$} \\
\hline & & & Lower & Upper & & \\
\hline Pre Base vs. Pre MS & 25.21 & 33.24 & 6.81 & 43.62 & .011 & 15 \\
\hline Post Base vs. Post MS & 22.72 & 39.54 & .825 & 44.62 & .043 & 15 \\
\hline Pre MS vs. Post MS & -2.49 & 20.12 & -13.63 & 8.65 & 639 & 15 \\
\hline
\end{tabular}

Table D.80. Post-hoc paired t-test of condition interaction CBF (\%) responses to mental stress for prehypertensive group, fish oil.

\begin{tabular}{lcccccc}
\multicolumn{1}{c}{ Variable } & Mean & Std. Dev. & \multicolumn{2}{c}{$95 \%$ Confidence Interval } & Sig. & N \\
\hline Pre Base vs. Pre MS & 29.87 & 31.63 & 10.75 & 48.99 & .005 & 13 \\
Post Base vs. Post MS & 19.12 & 25.25 & 3.86 & 34.38 & .018 & 13
\end{tabular}

Table D.81. Post-hoc paired t-test of condition interaction CBF (\%) responses to mental stress for prehypertensive group, placebo.

\begin{tabular}{lcccccc}
\multicolumn{1}{c}{ Variable } & Mean & Std. Dev. & \multicolumn{2}{c}{$95 \%$ Confidence Interval } & Sig. & N \\
& & & Lower & Upper & Sig. & \\
\hline Pre Base vs. Pre MS & 23.42 & 23.08 & 6.91 & 39.94 & .011 & 10 \\
Post Base vs. Post MS & 22.95 & 23.57 & 6.09 & 39.81 & .013 & 10
\end{tabular}

Table D.82. Post-hoc paired t-test of condition interaction CBF (\%) responses to mental stress for combined-group, placebo.

\begin{tabular}{|c|c|c|c|c|c|c|}
\hline \multirow{2}{*}{ Variable } & \multirow{2}{*}{ Mean } & \multirow{2}{*}{ Std. Dev. } & \multicolumn{2}{|c|}{ 95\% Confidence Interval } & \multirow{2}{*}{ Sig. } & \multirow{2}{*}{$\mathrm{N}$} \\
\hline & & & Lower & Upper & & \\
\hline Pre Base vs. Pre MS & 45.61 & 40.33 & 30.82 & 60.40 & .000 & 31 \\
\hline Post Base vs. Post MS & 26.67 & 28.67 & 15.41 & 37.22 & .000 & 29 \\
\hline Pre MS vs. Post MS & -15.77 & 26.20 & -25.93 & -5.61 & .002 & 29 \\
\hline
\end{tabular}


Table D.83. Normotensive repeated measures ANOVA of changes in 5 minute average $\Delta$ CVR (\%) in Study 2, 2 time (pre/post) vs. 2 condition (base/mental stress) vs. 2 Drug (fish oil/placebo), presented as one-tailed significance.

\begin{tabular}{lc}
\multicolumn{1}{c}{ Interaction } & Base - Mental Stress \\
\hline Time & .011 \\
Time x Drug & .218 \\
Condition & .023 \\
Condition x Drug & .198 \\
Time x Condition & .011 \\
Time x Condition x Drug & .218
\end{tabular}

Table D.84. Prehypertensive repeated measures ANOVA of changes in 5 minute average $\mathbf{\Delta C V R}$ (\%) in Study 2, 2 time (pre/post) vs. 2 condition (base/mental stress) vs. 2 Drug (fish oil/placebo), presented as one-tailed significance.

\begin{tabular}{lc}
\multicolumn{1}{c}{ Interaction } & Base - Mental Stress \\
\hline Time & .403 \\
Time x Drug & .390 \\
Condition & .441 \\
Condition x Drug & .285 \\
Time x Condition & .403 \\
Time x Condition x Drug & .390
\end{tabular}

Table D.85. Combined-groups repeated measures ANOVA of changes in 5 minute average $\Delta$ CVR (\%) in Study 2, 2 time (pre/post) vs. 2 condition (base/mental stress) vs. 2 Drug (fish oil/placebo), presented as one-tailed significance.

\begin{tabular}{lc}
\multicolumn{1}{c}{ Interaction } & Base - Mental Stress \\
\hline Time & .107 \\
Time x Drug & .274 \\
Condition & .088 \\
Condition x Drug & .487 \\
Time x Condition & .107 \\
Time x Condition x Drug & .274
\end{tabular}


Table D.86. Post-hoc paired t-test of condition interaction CVR (\%) responses to mental stress for normotensive group, fish oil.

\begin{tabular}{lcccccc}
\multicolumn{1}{c}{ Variable } & Mean & Std. Dev. & \multicolumn{2}{c}{$95 \%$ Confidence Interval } & Sig. & N \\
\hline Pre Base vs. Pre MS & -15.13 & 25.09 & -29.02 & -1.23 & .035 & 15 \\
Post Base vs. Post MS & -6.46 & 20.52 & -17.82 & 4.91 & .243 & 15
\end{tabular}

Table D.87. Post-hoc paired t-test of condition interaction CVR (\%) responses to mental stress for normotensive group, placebo.

\begin{tabular}{lcccccc}
\multicolumn{1}{c}{ Variable } & Mean & Std. Dev. & \multicolumn{2}{c}{$95 \%$ Confidence Interval } & Sig. & N \\
& & & Lower & Upper & & \\
\hline Pre Base vs. Pre MS & -6.70 & 16.35 & -15.75 & 2.36 & .135 & 15 \\
Post Base vs. Post MS & -2.28 & 22.38 & -14.68 & 10.11 & .699 & 15
\end{tabular}

Table D.88. Normotensive repeated measures ANOVA of changes in 5 minute average $\Delta$ CVC (\%) in Study 2, 2 time (pre/post) vs. 2 condition (base/mental stress) vs. 2 Drug (fish oil/placebo), presented as one-tailed significance.

\begin{tabular}{lc}
\multicolumn{1}{c}{ Interaction } & Base - Mental Stress \\
\hline Time & .003 \\
Time x Drug & .036 \\
Condition & .002 \\
Condition x Drug & .180 \\
Time x Condition & .003 \\
Time x Condition x Drug & .036
\end{tabular}

Table D.89. Prehypertensive repeated measures ANOVA of changes in 5 minute average $\triangle$ CVC $(\%)$ in Study 2, 2 time (pre/post) vs. 2 condition (base/mental stress) vs. 2 Drug (fish oil/placebo), presented as one-tailed significance.

\begin{tabular}{lc}
\multicolumn{1}{c}{ Interaction } & Base - Mental Stress \\
\hline Time & .243 \\
Time x Drug & .116 \\
Condition & .046 \\
Condition x Drug & .428 \\
Time x Condition & .243 \\
Time x Condition x Drug & .116
\end{tabular}


Table D.90. Combined-groups repeated measures ANOVA of changes in 5 minute average $\Delta$ CVC (\%) in Study 2, 2 time (pre/post) vs. 2 condition (base/mental stress) vs. 2 Drug (fish oil/placebo), presented as one-tailed significance.

\begin{tabular}{lc}
\multicolumn{1}{c}{ Interaction } & Base - Mental Stress \\
\hline Time & .004 \\
Time x Drug & .017 \\
Condition & .000 \\
Condition x Drug & .178 \\
Time x Condition & .004 \\
Time x Condition x Drug & .017
\end{tabular}

Table D.91. Post-hoc paired t-test of condition interaction CVC (\%) responses to mental stress for normotensive group, fish oil.

\begin{tabular}{|c|c|c|c|c|c|c|}
\hline \multirow{2}{*}{ Variable } & \multirow{2}{*}{ Mean } & \multirow{2}{*}{ Std. Dev. } & \multicolumn{2}{|c|}{$95 \%$ Confidence Interval } & \multirow{2}{*}{ Sig. } & \multirow{2}{*}{$\mathrm{N}$} \\
\hline & & & Lower & Upper & & \\
\hline Pre Base vs. Pre MS & 36.29 & 41.82 & 13.13 & 59.44 & .005 & 15 \\
\hline Post Base vs. Post MS & 16.14 & 27.95 & .659 & 31.61 & .042 & 15 \\
\hline Pre MS vs. Post MS & -20.15 & 24.61 & -33.78 & -6.52 & .007 & 15 \\
\hline
\end{tabular}

Table D.92. Post-hoc paired t-test of condition interaction CBF (\%) responses to mental stress for normotensive group, placebo.

\begin{tabular}{lcccccc}
\multicolumn{1}{c}{ Variable } & Mean & Std. Dev. & \multicolumn{2}{c}{$95 \%$ Confidence Interval } & Sig. & N \\
\hline Pre Base vs. Pre MS & 14.55 & 29.22 & -1.63 & 30.73 & .074 & 15 \\
Post Base vs. Post MS & 10.42 & 37.22 & -10.19 & 31.03 & .297 & 15 \\
Pre MS vs. Post MS & -4.13 & 20.52 & -15.49 & 7.23 & .449 & 15
\end{tabular}

Table D.93. Post-hoc paired t-test of condition interaction CVC (\%) responses to mental stress for prehypertensive group, fish oil.

\begin{tabular}{lcccccc}
\multicolumn{1}{c}{ Variable } & Mean & Std. Dev. & \multicolumn{2}{c}{$95 \%$ Confidence Interval } & Sig. & N \\
\hline Pre Base vs. Pre MS & 12.10 & 28.84 & -5.33 & 29.53 & .156 & 13 \\
Post Base vs. Post MS & 3.74 & 23.41 & -10.40 & 17.89 & .575 & 13
\end{tabular}


Table D.94. Post-hoc paired t-test of condition interaction CBF (\%) responses to mental stress for prehypertensive group, placebo.

\begin{tabular}{lcccccc}
\multicolumn{1}{c}{ Variable } & Mean & Std. Dev. & \multicolumn{2}{c}{$95 \%$ Confidence Interval } & Sig. & N \\
& & & Lower & Upper & & \\
\hline Pre Base vs. Pre MS & 6.22 & 18.53 & -7.04 & 19.48 & .316 & 10 \\
Post Base vs. Post MS & 8.46 & 17.17 & -3.82 & 20.74 & .154 & 10
\end{tabular}

Table D.95. Post-hoc paired t-test of condition interaction CBF (\%) responses to mental stress for combined-group, fish oil.

\begin{tabular}{lcccccc}
\multicolumn{1}{c}{ Variable } & Mean & Std. Dev. & 95\% Confidence Interval & Sig. & N \\
\hline Pre Base vs. Pre MS & 28.41 & 37.91 & 14.50 & 42.31 & .000 & 31 \\
Post Base vs. Post MS & 10.15 & 25.79 & .337 & 19.96 & .043 & 29 \\
Pre MS vs. Post MS & -14.68 & 24.19 & -24.06 & -5.29 & .003 & 29
\end{tabular}

Table D.96. Post-hoc paired t-test of condition interaction CBF (\%) responses to mental stress for combined-group, placebo.

\begin{tabular}{|c|c|c|c|c|c|c|}
\hline \multirow{2}{*}{ Variable } & \multirow{2}{*}{ Mean } & \multirow{2}{*}{ Std. Dev. } & \multicolumn{2}{|c|}{ 95\% Confidence Interval } & \multirow{2}{*}{ Sig. } & \multirow{2}{*}{$\mathrm{N}$} \\
\hline & & & Lower & Upper & & \\
\hline Pre Base vs. Pre MS & -11.68 & 23.75 & 2.81 & 20.55 & .012 & 30 \\
\hline Post Base vs. Post MS & -8.52 & 29.59 & -3.19 & 20.22 & .147 & 27 \\
\hline Pre MS vs. Post MS & -1.58 & 18.95 & -9.41 & 6.24 & .680 & 27 \\
\hline
\end{tabular}

Table D.97. Combined-group regression analyses of $\triangle$ MSNA (bursts/min) with $\triangle M A P$ and Resting HR following fish oil supplementation.

\begin{tabular}{cl|c} 
& Variable & DMSNA (bursts/min) \\
\hline $\begin{array}{c}\Delta \text { MAP } \\
\text { (mmHg) }\end{array}$ & Pearson Correlation & .354 \\
& Sig. (one-tailed) & .035 \\
& N & 27 \\
Resting HR & Pearson Correlation & \\
(beats/min) & Sig. (one-tailed) & -.466 \\
& $\mathrm{~N}$ & .007 \\
&
\end{tabular}


Table D.98. Combined-group regression analyses of $\triangle$ MSNA (bursts $/ \mathrm{min}$ ) with $\triangle M A P$ and Resting HR following placebo supplementation.

\begin{tabular}{|c|c|c|}
\hline \multicolumn{2}{|c|}{ Variable } & $\Delta$ MSNA (bursts/min) \\
\hline $\begin{array}{c}\triangle M A P \\
(\mathrm{mmHg})\end{array}$ & $\begin{array}{l}\text { Pearson Correlation } \\
\text { Sig. (one-tailed) } \\
\mathrm{N}\end{array}$ & $\begin{array}{c}-.036 \\
.860 \\
27\end{array}$ \\
\hline $\begin{array}{l}\text { Resting HR } \\
\text { (beats/min) }\end{array}$ & $\begin{array}{l}\text { Pearson Correlation } \\
\text { Sig. (one-tailed) } \\
\text { N }\end{array}$ & $\begin{array}{l}-.227 \\
.123 \\
28\end{array}$ \\
\hline
\end{tabular}

Table D.99. Combined-group regression analyses of $\triangle M S N A$ (bursts/100hb) with $\triangle M A P$ and Resting HR following fish oil supplementation.

\begin{tabular}{|c|c|c|}
\hline \multicolumn{2}{|c|}{ Variable } & $\Delta$ MSNA (bursts $/ 100 \mathrm{hb}$ ) \\
\hline $\begin{array}{c}\triangle \mathrm{MAP} \\
(\mathrm{mmHg})\end{array}$ & $\begin{array}{l}\text { Pearson Correlation } \\
\text { Sig. (one-tailed) } \\
\mathrm{N}\end{array}$ & $\begin{array}{l}.325 \\
.049 \\
27\end{array}$ \\
\hline $\begin{array}{l}\text { Resting HR } \\
\text { (beats/min) }\end{array}$ & $\begin{array}{l}\text { Pearson Correlation } \\
\text { Sig. (one-tailed) } \\
\text { N }\end{array}$ & $\begin{array}{c}-.429 \\
.013 \\
27\end{array}$ \\
\hline
\end{tabular}

Table D.100. Combined-group regression analyses of $\triangle$ MSNA (bursts/100hb) with $\triangle M A P$ and Resting HR following placebo supplementation.

\begin{tabular}{cl|c} 
& Variable & $\Delta$ MSNA (bursts/100hb) \\
\hline & & -.062 \\
$\Delta$ MAP & Pearson Correlation & .758 \\
$(\mathbf{m m H g})$ & Sig. (one-tailed) & 28 \\
& $\mathrm{~N}$ & \\
& & -.219 \\
Resting HR & Pearson Correlation & .131 \\
(beats/min) & Sig. (one-tailed) & 28 \\
& $\mathrm{~N}$ &
\end{tabular}




\title{
Appendix E
}

\author{
Request for Permission to Reproduce \\ Previously Published Material \\ (please save this file to your desktop, fill out, save again, and e-mail to permissions@the-aps.org)
}

Your Name: Chr1stopher e. Schwartz

E-mail: ceschwaremtu.edu

Affiliation: M1ch1gan Technolog1cal Un1vers1ty

University Address (for PhD students): 1400 Townsend Dr

Houghton, MI 49931

Description of APS material to be reproduced (check all that apply):
$\square$ Figure
$\square$ Partial Article
$\square$ Abstract
$\square$ Table
$\square$ Full Article
$\square$ Book Chapter
Other (please describe):

Are you an author of the APS material to be reproduced?

$\odot$ Yes

O No

Please provide all applicable information about the APS material you wish to use:

Author(s): Chr1stopher B. Schwartz, John J. Durocher, Jagon R. Carter

Article or Chapter Title: Neurovascular responses to mental stress in prehypertensive humans

Journal or Book Title: Journal of Applied Phys1ology

Volume: 110

Page No(s): $76-92$

Figure No(s): $1-4$

Table No(s): $1-2$

Year: 2011

DOI: $10.1152 /$ Japplphys101.00912.2010

(If you are reproducing figures or tables from more than one article, please fill out and send a separate form for each citation.)

Please provide all applicable information about where the APS material will be used:

How will the APS material be used? (please select from drop-down list) Dissertation

If "other," please describe:

Title of publication or meeting where APS material will be used (if used in an article or book chapter, please provide the journal name or book title as well as the article/chapter title):

Omega-3 ratty ac1ds and neurovascular responses to mental stress in humans.

Publisher (if journal or book): Proquest/unI

URL (if website):

Date of Meeting or Publication: Summer 2011

Will readers be charged for the material:

$\odot$ Yes

O No

APPROVED

By pripka at 11:01 am, Jun 22, 2011

Additional Information:

THE AMERICAN PHYSIOLOGICAL SOCIETY
9650 Rockville Pike, Bethesda, MD 20814-3991

Permission is granted for use of the material specified above, provided the publication is credited as the source, including the words "used with permission."

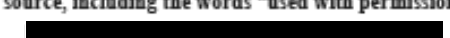

